\title{
ACTA ORIENTALIA
}

EDIDERUNT

\section{SOCIETATES \\ ORIENTALES DANICA NORVEGICA SVECICA}

(LE MONDE ORIENTAL)

CURAN'TIBUS SøREN EGEROD, HAVNIE,

NILS SIMONSSON, OSLO, H.S. NYBERG, UPSALIAE

REDIGENDA GURAVIT

SØREN EGEROD

AD IUVIT

ULLA KASTEN

VOL. XXXIV

APUD EJNAR MUNKSGAARD

HAVNIE

1972 
21. "Er, der die Opfergabe trägt, der Todlose ${ }^{31}$ (und) $" A$ gni ist der hotar, der purohila $\ll^{32}$ - dies sind die beiden samyäjy $\bar{a}^{33}$ Verse beim Sviștalyt ${ }^{34}$; nachdem sie gesprochen wurden, bringe man die beiden Spenden an Svișlakṛt dar.

22. Überall ${ }^{35}$ bei der Anführung einer Gottheit (erfolgt) die Weglassung der sonst ${ }^{36}$ iblichen (Gottheiten).

23. Auch an die Stelle der Gottheiten zwischen Sviștalịt und den beiden äjyabhägas (treten die vikp̣li-Gotheiten).

24. Diese Regel (gill auch bei Riten, die) den gleichen Zweck ${ }^{37}$

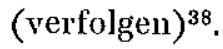

25. Beim zweiten (angehängten iș $i-\mathrm{Opfer}^{39}$ treten) die beiden vịdhanvanl-Sprïche ${ }^{40}$ (ein. Die Gottheiten sind) Agni Pāvaka und Agni Śuci. (Der hotar rezitiert als anuwäkyā und yäjyā fïr Aggni Pāvaka:) ${ }^{41}$ »Uns, o Reiniger, Strahlender « ${ }^{42}$ (und) »O Agni, Reiniger, mit Glanz« ${ }^{43}$; (für Agni Suci:) ${ }^{41}$ »Agni, herrlichstes Werk verrichtend «"4 (und) "Aufwärts, o Agni, deine leuchtenden . . . $\ll^{45}$.

26. (Beim svișlakr - Opfer $^{46}$ rezitiert er:) "Alle Widersacher besiegend $\ll^{47}$ (und) $\gg A g n i$ verehre ich, den purohila $\aleph^{48}$; so

31 ŖV III, 11, 2-

32 RV III, 11, 1.

${ }^{33}$ Diese nehmen Jem Opfer an Agni Sviștakṛt die Stelle von yäjyā und anuväkyă ein.

31 Eine Erscheinungsform des Gottes Agni und eine darauf bezügliche Opferhandlung.

${ }^{35}$ Nimlich bei den abgeleitelen Ritualformen (bilifli).

30 D. h. bei den Grundformen (prakrti).

37 ISomm.: samànajatìa ekakārya ilyarthah.

${ }^{38}$ Die sūtras 22-24, die algemeine Regeln enthalten, wïrten besser im Anschluß an II, 1, 6-8 stehen.

${ }^{39}$ Dieses sütra setzt II, 1, 18-21 fort.

40 S. I, 5, 35.

41 Diese Details nach dem Pray.

12 RV I, 12, 10.

43 T.V V $\mathrm{V}, 26,1$.

4. RY VIII, 44, 21.

45 RV VIII, 44, 17.

10 Pray.

17 R.V III, 11, 6.

48 R.V I, 1, 1 . 
lauten die sainyājyās ${ }^{49}$. - Beim dritten (angehängten iștiOpfer) fügt er vor dem vorletzten ${ }^{50}$ sāmidhenü-Vers ${ }^{51}$ die beiden $^{52}$ mil $»$ Der weithin Funkelnde, 'Todlose ${ }^{53}$ beginnenden Verse ein. ${ }^{54}$

27. Nachdem er die beiden eingeschobenen Strophen gesprochen hat, reziticre er die beiden gedeihenbringenden (Verse): "Durch Agni erlangte er Wohlstand ${ }^{55}$ (und) »Hausstandmehrer, Leidvernichter. ${ }^{56}$ (Die Gottheit) ist beliebig AgniSoma, (oder) Indra-Agni oder Vişnu.

28. (Oder) Aditi.

29. (Der hotar rezitiert für Aditi:) ${ }^{57}$ »Und auch dich, o große Aditi ${ }^{58}$, die große Mutter derer, die guten Wandels sind ${ }^{59}$, die Gattin des Rechts, wollen wir zu Hilfe rufen ${ }^{60}$, die Großmächtige, Nichtalternde, die sich weithin Exstreckende ${ }^{61}$, die guten Schutz gewährende Aditi, die gute Leitung gewährt. «62

30. (Der hotar rezitiert beim sviștakẹ $t^{03}$ ) $)$ Entflammt, o Agni« ${ }^{61}$ (und) »Diese, o Agni« ${ }^{85}$. Wenn die beiden samyājyăs im vira $a$-Metrum stehen sollen, dann verwende er diese (eben genannten). - Dies sind die drei (angeschlossenen ișliOpfer).

31. Oder es mögen nur das erste und das letzte (zur Anwendung kommen).

\footnotetext{
49 S. Anm. 33 (7u II, 1, 21).

50 Pray.: ultamasamipa upollamá tasyăh präk.

si S. I, 2, 7.

52 Nimlich RV III, 27, 5-6.

ss RV III, 27, 5.

54 Vgl. Ap VI, 31, 18; XIX, 18, 3.

os RV I, $1,3$.

86 RV I, 91, 12.

57 Dies geht aus dem Zusammenhang und auBerdem aus dem Pray. hervor.

58 RV VIII, 67, 10.

${ }^{59}$ Vgl. AV VII, 6, 2; TS I, 5, 11, 5; KS XXX, 4-5; MS IV, 10, 1; VS XXI, 5; AB I, 9, 7; Ãśv III, 8, 1; IV , 3, 2; ŚảnkhŚrS II, 2, 14; prattkas s. Ved. Concord.

60 S. Anm. 59 auBer $A B$ und $\AA s s$.

61 S. Anm. 60.

62 RV X, 63, 10 und Anm. 60.

63 Pray.

${ }^{61}$ praika aus preddho agne didihi puro nah RV VII, 1, 3.

${ }^{63}$ pratika aus ino agne vilatamāni havya $\mathrm{RV}$ VIT, 1, 18.
} 
32. Oder (nur) das erste.

33. Wenn dies (letztere) der Fall ist, (damn stehen) die beiden darin eingeschobenen Verse ${ }^{66}$ im vira $\bar{j}$-Metrum.

34. Bei einer solchen Umwandlung gehe er $^{07}$ (also) nach virajjMuster vor.

35. Nach dem Anlegen (der heiligen Feuer sind diese) zwölf Nächte lang unaufhörlich (zu unterhalten).

36. (Das Feuer) eines gatasri ${ }^{08}$ aber (ist) bis zu dessen Lebensende $^{69}$ (zu unterhalten) ${ }^{70}$.

Ende der 1. kaṇçlikä

II, 2

1. Nach dem (Vollzug des eben geschilderten) Rituals ${ }^{71}$ läßt ${ }^{72}$ man am Nachmittag den gärhapalya ${ }^{73}$ aufflammen. Das Südfeuer ${ }^{74}$ holt man von einer reichen Familie des dritten varna. Oder, wie einige meinen, beide Feuer sind gleichen Ursprungs $^{75}$. Ein beständig (unterhaltenes Südfeuer) lasse man auflammen. Oder man quirle mit einem Feuerbohrer. (Dann) entnehme man dem gärhapatya Glut für den ăhavanīya ${ }^{76}$.

as dhayya; s. II, 1, 26.

67 Der holar.

ss Ein Mann, der im Rahmen seines varna alles uberhaupt Mögliche erreicht hat ; nach den Texten unter den Bralmanen ein mahäbrahmana, unter den $k$ şatriyas ein rajjanya, unter den vaisyas eln grämani.

"2 Komm.: alyantam yäuajizumilyarthah.

7o Hiernit schliebt die Darstellung des agnyādheya-Rituals.

7 Also nach der Errichtung der heiligen Feuer.

72 Nummeht erfolgt die Beschreibung des agnihotra, des morgendlichen und abendlichen Milchopfers.

${ }^{33}$ Eines der drei Opferfeuer, von kreisformigem Grundriß; vgl. TS $1,6,7,1$; KS VI, 4.8; KIKS IV, 3.7; MS I, 4, 10; VS III, 39; XIX, 18; AB III, 36-37; VII, 6 ; KB II, 1; PB V, 4, 13; XXV, 10, 4; ȘB I, 5; IV, 1; JB I, 17; TB I, 1, 5, 6; SB III, 6, 1, 28; VII, 1, 1, 6; GB I, 3, 10; SA X, 8; JUB IV, 26, 15; TA II, 3, 1; Ap VII, 7, 3; XI, 5, 10; IKäty I, 8, 34 .

7* dakșinăgni ( = anvăhäryapacana), von halmmondförmigen Grundriß; vgl. TS I, 6, 7, 1; MS I, 5, 14. 6, 11; ȘB IV, 1 ; 'TB I, 1, 8, 1; GB 1, 2, 5.22. 3, 3.13; CU IV, 17, 5 .

75 Vgl, zu dieser Divergenz noch $\AA_{p}$ VI, 2, 11; Íăly IV, 13, 4.

${ }^{76}$ Eines der drei Opferfeuer, von viereckigem Grundriß; vgl. 'TS II, 2, 5, 6. $6,3,2$; KS VI, 8; KISS IV, 7; MS I, 5, 14; AB V, 24.26.31-32.34; VII, 2.6-8. 
2. Man entnehme (die Glut mit folgenden Sprïchen:) »Dich, den Gott, entnehme ich den Göttern mit Glanz. «77

3. "Der du herausgenommen wirst, nimm mich hinweg ${ }^{78}$ von dem Bösen, das ich unwissentlich and das ich wissentlich begangen habe. ${ }^{79}$ Vor jeglicher am Tage begangenen Sünde ${ }^{8081}$ schülze als Herausgenommener mich $l \ll^{82}$ (Mit diesen Worten) bringe er (das Feuer zum ähavañy ${ }^{83}$ ).

4. »Die Unsterblichkeitsopferspende, das Feuer gieße ich auf der unsterblichen ${ }^{84}$ Erde in den Schoß der Unsterblichkeit. ${ }^{85}$ Dadurch will ich unendliche Wunscherfüllung crsiegen. ${ }^{80}$ Diese ersiegte Prajāpati zuerst. ${ }^{87}$ Zum Feuer das Feuer ${ }^{88}$ Svähäl« (Mit diesen Worten) lege er (das Feuer auf dem Platz des ahavanĩya ${ }^{89}$ ) nieder, das Antlitz der Sonne zugewandt.

5. IVird das morgendliche (agnihotra) vor Tagesanbruch (dargebracht), so ist (das Gesicht dem Ostpunkte ${ }^{20}$ ) zugewandt.

6. (In diesem Falle) bringe er aber (das Feuer zum ähavanīya mit dem Spruch:) "Was an Sünde ${ }^{91}$ nachts ${ }^{92}$."

12.17.21.23-24; VIII, 9.24; KB II, 1 ; PB IV, 9,1 ; V, 4, 1; XII, 10, 15-16; XIX, 13, 9 ; XXV, 10, 4; JB I, 17; SB I, 5 ; IV, 1; TB I, 1, 5, 3; SB I, 1, 1, 11. 3, 3, 12. $9,2,4$; II, $3,4,24 ; \mathrm{IV}, 5,7,6.6,8,5 ; \mathrm{XI}, 5,3,8 ; \mathrm{GBI}, 2,5 ;$ SA X, 8 ; JUB IV, 26, 15; TA X, 63-64; CU II, 24, 11; IV, 13, 1; S̄inikh III, 19, 4; Ảp VII, 7, 3 ; Iñty I, 8, 35.44; IV, 9, 10.

"Dieser Spruch ist ohne Parallele in der vedischen Literatur.

78 Vgl. Sañnkh II, 6, 6; Аp VI, 1, 7; Mān I, 6, 1, 3.

79 S. Anm. 78.

80 Nämlich vor deren bösen Folgen.

81 S. Amm. 78.

82 S. Anm. 78.

83 Konm.

81 Vgl. Sānnklı II, 6, 7; Ap VI, 1, 8; Mān I, 6, 1, 4 .

85 S. Anm. 84.

86 S. Anm. 84.

87 S, ebenfalls Anm. 84, doch weichen die genannten Stellen ju diesem mantra von $\lambda$ śv etwas stärker ab.

${ }^{88}$ Vgl. Käty V, 2, 6; Vaits VIII, 11; KauśS III, 16; GobhGṛS IV, 1, 13. Die in Amm. 84 genamten Stellen aus $A_{p}$ und Mān haben agnim agnaı svăhä.

89 Komm.

90 Komm.: der Opfernde steht westlich vom ahavaniya-Platz.

o1 Statt yadana der Sūtra-Ausgabe muB es yadena heißen.

${ }_{22}$ Nämlich in Abwandlung des mantras zu Amm. 81 in II, 2, 3. Vgl. Män I, 6, 1, 3. Vollständig rälryă yad enah krtam asli päpam; vgl. Āp VI, 1, 7. 
7. Von da ${ }^{93}$ an hat der Opferveranstalter ${ }^{94}$ sich dem Eid( ${ }^{95}$ entsprechend bis zum (eigentlichen) Opfer zu verhalten.

8. Und ein täglich Opfernder (hat sich so) bis zum Sommenaufgang (zu verhalten).

9. (Die andere) Opferung (hat) nach Somnenuntergang (zu erfolgen).

10. Das Mundausspülen ist obligatorisch ${ }^{96}$.

11. Nachdem er ${ }^{97}$ MMit Recht und Wahrheil besprenge ich dich ringsum « ${ }^{98}$ gemurmelt hat, beträufele er (die Ungebung der) drei (Feucr) dreimal, jerreils crneut (frisches) Vasser benutzend.

12. In der Reihenfolge (dieser Ritualhandlungen herrscht) freie Wahl.

13. Das Südfeuer jedoch ist als erstes anzusehen ${ }^{99}$ : fürwahr der Vater der Fener ist das Südfeuer, der Sohn der garhapalya, der Enkel der ăhavanīya; deshalb beträufele er so ${ }^{100}$.

14. Vom gärhapalya vollziehe er einen ununterbrochenen Wasserguß mit (dem Spruch) »Das Gewebe ${ }^{101}$ des Luftraums ausspannend, folge dem Glanze nach $\mid \ll^{102}$ bis zum ähavanĩya.

15. Nachdem er sich westlich rom gärhapalya niedergelassen hat, versetze er Kohlen in nördliche Richtung (und spreche:) "Von gutem Opfer bereitet seid ihr; ein gutes Opfer werdet ihr bereiten. « ${ }^{103}$

16. Auf diese setze er die Opfersubstanz (und spreche:) "Das Daraufgesetzte, Hinaufgesetzte, Daraufgesetzte, hizm. «10*

${ }^{93}$ Nämlich von dem vor Tagesanbruch erfolgten Entzindlen des ahavaniya an.

94 Hier nicht yajamāna, sondern ahitagni uder die heiligen Feuer angelegt hat,.

95 Komm, und Pray. sind hier mit satridharmāścaturmäsyauratāni wenig präzis.

96 Vgl. GB I, 1, 39 .

97 Der Priester.

98 Vgl. Mần I, 6, 1, 10.

- Im Folgenden wird der Sūtra-Stil plötzlich der Brăhmaṇa-Diktion, d. h. einem arthavāda ztliebe, aufgegeben.

100 D. h. in der angegebenen Reihonfolge.

101 Es ist tantum zu leselr.

102 RV X, 53, 6; vgl. Asv $1,11,9$.

103 Der Spruch in dieser Form nur hier; vgl. II, 3, 3.

${ }^{104}$ Dieser eigenartige Spruch vom Charakter einer Zauberformel hat in der vorliegenden Form keine Parallele. 
17. Oder (er spreche:) "Der Iḍa ${ }^{105}$ Fußspur ist mit Schmelzbutter versehen und beweglich ${ }^{108}$. O Jatavedas ${ }^{107}$, erfreue dich an dieser Opfergabe ${ }^{108}$. Die vielgestaltigen zahmen Tiere $^{103}$ - von sieben (Arten) derselben soll bei mir die Zucht ${ }^{10}$ (erfolgen), «

18. Saure Milch setzte man nicht (auf das Feuer). Einige (lehren :) man setze (sie unbedenklich) auf ${ }^{111}$.

Ende der 2. kaṇdikā

II, 3

1. Das Opfer ist obligatorisch mit einem Milchtrank (vor\%unehmen).

2. Reismilchschleim, Milchkornmus, saure Milch, zerlassene Butter (gelten respektive) für einen, der ein Dorf (zu beherrschen) winscht, - der Nahrung wünseht, - der Sinneskraft wünscht, - der Glanz ${ }^{12}$ wünseht.

3. Das (auf's Feuer) gesetzte (Opfergut) lasse er abbrennen.

4. (Das gilt auch für die) nicht aufgesetzte ${ }^{113}$ Milch ${ }^{114}$. (Dabei

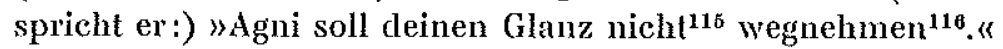

5. Wit einem Opferlöffel ${ }^{117}$ beträufele ${ }^{118}$ er je nach Wunsch

105. Vgl, I, 7, 7 und ZMR Anm, 248 .

106 Vgl, Ap VI, 5, 7; Mān I, 6, 1, 15.

107 Eine Bezeichnung des Gottes Agni.

108 Vgl, Amm. 106 sowie HirGrS II, 17, 2,

100 Vgl. Amm. 108 sowie AV II, 34, 4; III, 10, 6; MB II, 2, 14; TA III, 1, 11-12.

110 Vgl. AV III, 10, 6; TA III, 11, 12-; MB II, 2, 14; ferner $A$ p, Man und Hir

wie Anm. 108. puṣti ist hier wohl besser als "Zuchta, nicht schlechthin als "Gedeihen" aufzufassen.

111 Hiemtit schließen die elnfuhrenden Anweisungen ther das agnihotra. Die nächsle kandika wendet sich dann dem eigentlichen Opfervorgang zu.

112 tejas wäre hier vielleicht besser mit ${ }^{2}$ Einfluß $ß u$ ubersetzen, aber der Komm. hat tejaścchavih.

${ }_{113}$ D. h. wohl für zwar bereltgesteltes, aber ibrig gebliebenes Opfergut.

114 Hierbel steht der Ausdruck MMileh ftir Opfergut schlechthin (Komm.).

11 Statt mähärşĭh muB es má härşil heilßen.

116 Vgl, Mรān I, 2, 5, 15.

117 sriba.

118 Nach dem Komm. gllt dies, wenn Milch die Opferspeise 1st. Damn soll das Milchgefäß ausgewaschen und vom Waschwasser etwas in die Mfich geträufelt werden. 
(und spreche:) $\gg$ Frieden bist du119, Unsterblichkeit bist du ${ }^{120}$."

6. Bei diesen beiden (Möglichkeiten ${ }^{121}$ ) gibt es keine Vermischung ${ }^{22}$.

7. Mit dem erneut entflammten (Feuerbrand ${ }^{123}$ ) umrunde er dreimal (das Feuer und spreche:) $\gg$ Entfernt $^{124}$ ist der Dämon $^{125}$; entfernt ${ }^{124}$ sind die Feinde ${ }^{126}$."

8. Das (durch das Kochen) überquellende (Gefäß ${ }^{127}$ ) hebt er langsam ${ }^{128}$ empor (und spricht die drei Formeln:) "Dem Himmel dich ${ }^{129}$, dem Luftraum dich ${ }^{130}$, der Erde dich ${ }^{131}$ " (Beim dritten mantra ${ }^{132}$ ) setzt er es (auf die Erde ${ }^{133}$ ) nieder.

9. Mit "Von gutem Opfer bereitet seid ihr; ein gutes Opfer habt ihr bereitet ${ }^{134}$ schleudert er die Kohlen fort ${ }^{135}$. Den großen ${ }^{136}$ und den kleinen ${ }^{137}$ Opferlöllel erhitze er (und spreche dabei:) "Verbrannt ist der Dämon ${ }^{138}$, verbrannt

119 Vgl. MS IV, 9, 27; KB VI, 14; Śänkh IV, 7, 9; PārGṛ III, 16, 1.

120 Vgl. VS I, 31; IV, $18 ; \mathrm{X}, 15$; TS I, 2, 4, 1. 7, 9, 2. 8, 14, 1; III, 3, 3, 3. 4, 3; MS I, 1, 11. 2,4; ISS II, 5; PB XXI, 3, 7; 'TB I, 7, 8, 1; ŚB I, 3, 1, 28; III, 2, 4, 14; V, 4, 1, 14; GB I, 1, 39; Śănh IV, 8, 2; Äp XVIII, 6, 1.15, 5; XXXI, 17, 10; Kauś LXXX, 56 ; XC, 20.

121 Nämlieh zu beträufeln oder es zu unterlassen.

${ }^{123}$ D. h. nach Pray. und Konm., daß man es entweder immer tut oder nie.

${ }^{123}$ Nämlich dem, der ztm Erstanzinden des ahavantya gedient hat; Komm.: ulmuka; Pray.: trạa.

121 Partizip.

125 Vgl. TS I, I, 8, 1; VSIK I, 8, 3; JB I, 39; TB III, 2, 8, 5; Ap I, 25, 8; VI, 6,8 ; Mầ I, 6, 1, 20; Kăty II, 5, 22.

126 S. Anm. 125.

127 Komm, : sthati.

128 Komm.: mandagati.

${ }^{129}$ TS I, 1, 11, 1 und an zahlreichen anderen Stellen; Näheres s. in der Ved. Concordance.

130 S. Anm, 129.

13. S. Amm. 129 .

${ }^{132}$ So nach tem Komm.

199 S. Amm, 132.

13 V Vgl, Āśv II, 2, 15.

135 Nach den Komm, zurück in den gärhapatya.

136 sruc.

137 sruva.

${ }^{138}$ rakșas. 
sind die Feinde ${ }^{139}$; verglüht ist der Dämon ${ }^{140}$, verglüht sind die Feinde ${ }^{141}$."

10. Nachdem er ${ }^{142}$ den kleinen Opferlöflel nördlich vom $O_{p} f e r-$ gefäß deponiert hat, entsende er mit $» O \mathrm{O}$, ich will herausschöpfen (143 $^{140 n}$ Opferveranstalter ${ }^{144}$ ).

11. Nachdem er sich (den Mund) gespült hat, schreitet der Opferveranstalter entlang der Westseite der Opferstätte nach Süden $^{145}$. Nachdem er sich (dort) niedergelassen und das ${ }^{140}$ gehört hat, veranlasse er (den Priester zu seincr weiteren Tätigkeit) mit "Om, schöpfe heraus! «147

12. Der ausgeforderte (Priester) schöpfe (vier.148) Löllelvoll (Nilch) heraus (und spreche jeweils dazu:) $" B h \bar{a} r$ I $\mathbf{l}^{149}$, bhuva Icla $\vec{a}^{150}$, svar Idla $\vec{a}^{151}$, vrdha Id $\vec{a}^{152}$.

13. Einen jeden vorhergehenden ${ }^{153}$ (Löffel) fülle voller (als den folgenden), wer (seinen) Söhnen entsprechend ihrer Altersfolge Gedeihen wünscht.

14. Wer ihm als Sohn (besonders) lieb ist, für den schöple er einen (ganz) vollen (Löfiel).

15. Nachdem er das Opfergefäß berührt, ein Bremnholzstïck und den grossen Opferlöffel oberhalb des gārhapalya gehalten und (beides) in Nasenhöhe (getragen hat), setzt er es

139 'TS I, 1, 2, 1; im ibrigen s. Anm. 120.

140 Vgl. VS I, 7.29; SB I, 1, 2, 2. 3, 1, 4; Аp I, 12, 1; Mān I, 6, 1, 25; Katus III, 9.

141 S. Amm. 140 auBer $\AA_{p}$.

142 Der Priester, der bei einem relativ so einfachen Opfer wie dem agnihotra die Funktionen des hotar und des adhvaryı vereinigt. Die Prayogadipikā bezeichnet ihn hier als ksirahotar (mit der Opfermilch befaßter hotar) und deutet damit schon einen Synkretismus an, ebenso Käty IV, 14, 31.

${ }^{143}$ Vgl, ähnlich $\bar{\Lambda}$ p VI, 7, 1 .

14: Koomm. und Pray.: ahitägni.

$145 \mathrm{Im}$ Text ist kṣanata in dakşinata zu verbessern,

146 Nämlich dic letzte Bemerkung des Prlesters.

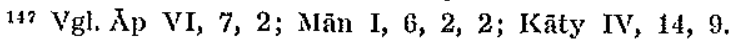

148 Komm, und Pray. Nach der Pray. schöpt er diese Mileh in die sruc.

149 Vgl. $\Lambda$ p VI, 8, 3; Măn I, 6, 1, 26 .

160 S. Anm. 149.

151 S. Anm. 149.

152 Nur Áśv.

${ }^{153}$ agniyamagniyam des Textes ist in agriyamagriyam zu verbessern. 
in der Nähe des ahhavanĩya auf kuśa $a^{154}$-Halmen nieder, schlägt (das rechte155) Knie ïber (das linke); (damn) lege er das Bremmholz in (den âhavanüya und spreche:) "Als silbern, feuerglänzend lege ich dich, die Nacht, als Ziegel $a^{150}$, svähā !«

16. Nachdem er das Bremnholzstück hineingelegt hat, und nachdem er mit $»$ Ein Blitz bist du ${ }^{157}$, tremne $a b$ meine Sünde ${ }^{158}$; im Fener jst Treue «159 Wasser berührt hat, gieße er zwei Daumenbreiten über dem entflammten (Holzstïck ${ }^{160}$ einen Milchstrom) aus (und spreche:) »Bhür bhuvah svah ozm ${ }^{161}$. Agni ist Licht, Licht ist Agni ${ }^{162}$. Svāhăl«

17. Nachdem er (diese) erste Spende dargebracht und (den großen Löflele ${ }^{163}$ ) auf die kuśa-Halme gelegt hat, betrachte ex den gārhapalya (und spreche:) »Bändige mir das Vieh!«104

18. Dann (soll der Priester) schweigend einen reichlicher (geschöpfen und vom Erstopfer ${ }^{105}$ ) unbeeinflußten (Löfelvoll) nordöstlich oder nördlich (davon ${ }^{166}$ darbringen).

19. An Prajāpati soll er im Geiste denken überall da, wo Schweigen $^{167}$ bei den Opfertungen (vorgeschrieben ist).

154 Das Gras Poa cynosuroides, in den Brähmayas darbha.

155 Nach dem Komm. und nicht urbedingt zuverlässig, zumal die Pray. hier keine Erklärung gibt.

156 Vgl. annähernd $\overline{A p}$ VI, 9, 4.

157 Vgl. TB III, 10, 9, 2; Ap IV, 1, 6; VI, 5, 3. 9, 3; XIX, 13, 19; Ināty IV, 15,5 .

158 S. Anm. 157 sowie Sinnkh II, 7, 18; IV, 8, 3.

159 Nur bei $\bar{A} s$.

160 Komm.: tiber der Wurzel.

$161 \mathrm{Vgl.} \mathrm{AB} \mathrm{V,} \mathrm{31,} \mathrm{4;} \mathrm{VIII,} \mathrm{27,} \mathrm{4;} \mathrm{TA} \mathrm{X,} \mathrm{2,} \mathrm{1.} \mathrm{15,} \mathrm{1.} \mathrm{27,} \mathrm{1.} \mathrm{28,} \mathrm{1;} \mathrm{Ásv} \mathrm{I,} \mathrm{2,} \mathrm{3;}$

II, 4, 25; Sān̉kh IV, 6, 9; Măn V, 2, 15, 10. 16, 14; AśvGṛS I, 14, 4; MB I, 6, 30;

II, 4, 5.14; BaudlıDhS II, 7, 12, 2.5.

162 Vgl. SV II, 1189; MS I, 6, 10. 8, 1.5; II, 7, 16; KS XL, 6; VS III, 9; AB

II, 31, 4. 32, 1. 37,17 ; V, 31, 4; IKB II, 8; XIV, 1 ; JB I, 4; ŞB I, 4, 9; TB II, $1,9,2$; SB II, 3, 1, 30.32.36; TA IV, 10, 5; V, 8, 10; Asv V, 9, 11; Sănkh II, 9, 1 ; VII, 9, 2; LățySrS I, 8, 14; $\lambda_{p}$ VI, 10, 8; XV, 12, 8; XVI, 23, 10; Mān I, 6, 1, 37.

163 Komm, und Pray.

164 Oder: halte fest bzw. zügele mir das Vieh; vgl. KS VI, 4; VII, $14 ; \AA \mathrm{p}$ VI,

8, 4; Mān I, 6, 1, 28.

ies Komm. und Pray.

168 Von der Stelle des Erstopfers.

167 Etwas frei übersetzt. 
20. Das meiste ${ }^{168}$ läßt er im großen Löflel übrig, schüttelt es dreimal ${ }^{109}$, wischt ab und schältelt (den Rest ${ }^{170}$ ) auf die laśa-Wurzeln nieder (wobei er spricht:) "Dem Vieh dich! «171

21. Südlich derselben ${ }^{172}$ hält er die Handlächen nach oben. Ev trägt die Brahmanenschmur ${ }^{173}$. Entweder schweigt er Oder (er spricht:) Svadha $a^{174}$ den Ahnen! «175

22. Nachdem er Wasser ausgegossen hat, -

23. berühre er Wasser (und spreche:) $\gg$ Regen bist du ${ }^{176}$, tilge meine Sïnde ${ }^{177}$; in den Wassern ist Treue. ${ }^{178}$

24. Der Opferveranstalter rede ${ }^{179},-$

25. nachdem er die ädhäna (-Formel ${ }^{180}$ ) gesprochen hat, das Bremnholzstïck an mit »Durch diesen rssi, durch diesen heiligen Spruch, durch diese Gottheit sitze fest in Angiras ${ }^{181}$ Veise $1 \ll^{182}$

26. Mit $"$ Die süße Milch gebenden «183 (redet er) den ersten Opferguß (an). . $^{184}$

168 Von der beim Zweitopfer geschöpften Substanz.

168 Komm. und Pray.: uber dem ahavaniya. Komm. und Pray, geben zu diesem sülra noch eine Reihe weiterer Details.

170 Von der Hand.

17 Vgl. TS III, 5, 2, 4; IV, 4, 1, 2; KS XVII, 7; XXXVII, 17; 1'B I, 10, 3; GB II, 2, 13; Vail XXV, 1.

172 Der kuśá-Wurzeln.

173 D. h. ex ist ein präcinâvitin und trïgt die heilige Schnur aber der rechten Schulter und unter dem linken Arm; vgl. TB I, 4, 6, 6. 6, 8, 2. 9, 3; SB II, 4, 2, 2.9. $6,1,8 ;$ XII, 5, 1,6 .

17 Ein an die Ahmen gerichteter Ruf, etwa oWohlgefalten.

175 Vgl. TS I, 1, 11, 1. 3, 4, 2; VI, 3, 2, 5; MS I, 2, 13; III, 9, 1; ISS III, 1; XXVI, 2; VS II, 7; TB III, 3, 6, 4; SB I, 4, 5, 1; TA X, 2-4.6; $\AA_{p}$ II, 8, 3; VI, 11, 4; XI, 18, 2; Kāty III, 1, 15; IV, 14, 21; Kaus LXXIII, 6; ÃśvG̣̣S I, 2, 10. 176 Vgl. TB III, 10, 9, 2; $\lambda \leqslant s$ V, 3, 15; Sănkh II, 7, 18; IV, 8, 3; Ap IV, 16, 16; VI, 11, 4. 14, 6; XIX, 13, 21; Käty IV, 15, 4.

177 S. Anm, 176 außer Asv V.

$178 \mathrm{Vgl} . \mathrm{Ap}$ VI, $14,6$.

170 Es würde diles besser zwischen sütra 15 und 16 passen.

180 Es kann sich, zumal die Pray, de Vorschrift oohne suahäa gibt, nur un die Formel am Schluß von II, 3, 15 handeh.

181 Name einer Priesterfamilie, oft als mythisch zu betrachten.

182 Vgl. 'IS IV, 4, 6, 2; MS IV, 9, 15-16; TB III, 12, 6, 1.6. 7, 1.5. 8, 1.3; Āp $\mathrm{XVI}, 28,1$.

t89 Vgl. RiV VIII, 69, 3.

18 Dies wïrde eher nach sūtra 16 passen. Aber der sütrakära bevorzugt nicht 
27. Nachdem er ${ }^{185}$ sich erhoben hat, erwarte er hinschauend den zweiten (Opferguß mit:) »Bhür bhuvah suah180 Möchte ich durch Nachkommen gute Nachkommen besitzen, durch Helden gute Helden, durch Gedeihen gutes Gedeihen $\mid$ ¿187 $^{18}$

28. Und mit (drei ${ }^{188}$ ) an Agni gerichteten (Sprüchen), -

29. (sowie) mit den drei mit $" A g n i$, du läuterst die Leben «189 (begimmenden Sprüchen). ${ }^{100}$

Ende der 3. kaṇḍikä

II, 4

1. Diese ${ }^{191}$ (sind) Jahr für Jahr ${ }^{192}$ (zu gebrauchen).

2. Mit Reis- (bzw. Gersten-) Milchschleim oder mit Milch opfere er $^{193}$ selbst am Tage des Mondwechsels.

3. Einer der Priester ${ }^{194}$ (darf auch fär sich selbst) eine andere Zeit wählen.

4. Auch ein Brahmanensehüler ${ }^{195}$ (darf das).

5. Nachdem er Wasser ber'üht und sich nach Norden gewandt hat, soll er (den Opferrest) verzehren.

6. Oder (er esse erst dann), wenn er auf den beiden anderen (Feuern ${ }^{190}$ ) geopfert hat.

die synoptische Darstellung, sondern behandelt die Pflichten vou Priester und ähitägni getremnt.

185 Der Opferveranstalter.

186 S. ZMR, Anm. 446.

${ }^{187}$ Vgl. VS III, 37; VII, 29; VSK IIX, 4, 1; IX, 1, 5; SB IV, 5, 6,4; Sīnikh

II, 13, 2. Abwandlungen dieser Formel zeigen noch einige andere Stellen.

${ }^{188}$ So Komm. und Pray.

${ }^{189}$ RV IX, 66, 19; s.a. Anm. 29 zu II, 1, 20.

100 Die Pray. läßt die in sūtra 28 erwähnten Spriiche mit upa tvägne dive-dive (RV I, 1, 7) beginnen und für den täglichen Gebrauch obligatorisch sein, während die Sprutche in sūtra 29 sambalsare samvalsare zu verwenden seien, s. Āśv II, 4, 1. - Die kandikä-Teilung ist an dieser Stelle wenig simnvoll,

191 S. II, 3, 29.

192 Nach dem IKomm.: eimmalig jeweils am Jahresende.

103 Der Opferveranstalter.

104 Gemeint ist schlechthin: ein Priester (rtvij).

193 antevasin; es handelt sich um einen brahmacärin, der im Hause des Lehrers lebt; vgl, JB I, 296; SB V, 1, 5, 17; SA XI, 8 ; TA VI, 3, 3. 11, 1 ; BU VI, 3, 15-20; CU III, 11, 5; IV, 10, 1 .

${ }^{196}$ gärhapalya und dakșināañi. 
7. Beim ersten Mal (speist er mit:) »Zum Leben verzehre ich dich ${ }^{197}$; beim zweiten Mal (mil:) $» Z u r$ Nahrung dich. « ${ }^{198}$

8. Schweigend (ergreift er) ein Brennholzscheit und legt es mit "Agni, dem Hausvater, Heil 1 (199 $^{19}$ den gärhapalya.

9. Die zweite (Opferspende vollzieht er) grundsälzlich (schweigend $\left.{ }^{200}\right)$.

10. Schweigend (ergreift er) ein Brennholzscheit und legt es mit "Agni ${ }^{201}$, dem Hern des Niederlegens ${ }^{202}$, Heill « ${ }^{203}$ oder mit "Agni, dem speisegewährenden Speiseherm, Heil! ${ }^{204}$ in das Südfeuer.

11. Die zweite (Opferspende vollzieht er) grundsätzlich (schweigend $\left.{ }^{205}\right)$.

12. Nachdem er gegessen hat, gießt er in Richtung auf sich selbst mit dem großen Löffel dreimal Wasser aus (und spricht:) $»$ Den Schlangengötterscharen Heill « ${ }^{206}$

13. Nachdem er ihn ${ }^{207}$ mit kuśa (-Halmen) gesäubert hat, gieße er vicr Löllelvoll ${ }^{208}$ nach Nordosten aus (mit den vier Sprüichen:) „Den Jahreszeiten Heill «209, »Den Himmelsrichtungen Heill $\ll^{210}$, »Den sieben $r$ șis Heill ${ }^{211}, ~ » D e n$ anderen Wesen Heill $^{212}$

107 In dieser form nur hier.

$108 \mathrm{Vgl}$. TS I, 7, 9, 2; Ap XVIII, 5, 17.

199 Vgl. TS I, 8, 15, 2. 16, 2; MS II, 6, 13; IV, 4, 7; KS XV, 8; VS X, 23; TB

I, 7, 10, 6; SB V, 4, 3, 15; Śānkh II, 10, 1; $\bar{A}_{\mathrm{p}}$ XVIII, 17, 14. 20, 4; Män IX, 1, 5. 200 Komm. und Pray.

201 Dieser und der folgende Spruch fehlen in der Ved, Concordance.

202 samiveśa bedentel nach R. T. H. Griffths hier das Beilager.

203 Vgl. VS II, 20.

204 Vgl. VSK III, 2, 5; AB VII, 12, 5; Kăty IV, 14, 25; Vail. VII, 19.

aos Wie in sütra 9 ; nur handelt es sich hier nicht um den gặrhapatya, sondern um das Südfeuer.

206 Dieses mantra nur hier. Statt sarvadevajana liest man mil Pray. und ĀśvGṛS

II, 1, 9.14 besser sarpa $a^{0}$. Diese Lesart wird auch durch CU VII, 1, 2.4. 2, 1. 4,

1. 7,1 gestiutzt.

207 Deln großen Löflel.

208 Wohl mit Wasser (im Anschluß an das vorhergehende sütra), doch Genaues wird nirgends gesagt.

209 Vgl. IS VII, 1, 15, 1; MS III, 12, 7; VS XXII, 38; TB III, 1, 6, 1.

210 Vgl. TS VII, 1, 15, 1; MS III, 12, 7; VS VI, 19; XXII, 27; XXXIX, 2;

SB II, 4, 4, 24; III, 8, 3, 35; XIV, 3, 2, 10; BU VI, 3, 6.

211 Dieser Spruch nur hier.

212 Vgl. MIān I, 6, 1, 47. 
14. Den fünften (Löffelvoll gieße er) auf die kuśa-bedeckte Fläche (mit:) "Auf die Erde gieße ich den Nektar ${ }^{213}$; dem Agni Vaiśvănara Heill « ${ }^{214}$ Den sechsten (Löffelvoll gieße er) westlich des gärhapalya (aus mit:) $»$ Den Atem gieße ich in den Nektar aus ${ }^{215}$; den Nektar gieße ich in den Atem aus ${ }^{216}$. Svāhă!«

15. Nachdem er (den großen Löffel ${ }^{217}$ ) erhitzt ${ }^{218}$ hat, lege er (ihn) innerhalb der Opferstätte nieder.

16. Oder er übergebe ihn einem Diener.

17. Nachdem er am Rande des ähavanüya entlang gegangen ist $^{219}$, lege er, mit dem Gesicht nach Norden gewandt stehend, jeweils drei Holzscheite hinein ${ }^{220}$, -

18. den ersten (immer) mit einem Spruch.

19. (Beim Einlegen) in den ähavanìya mit "Glänzel ${ }^{221}$, in den gārhapalya mit $» \mathrm{Er}$ glänzte ${ }^{222}$, in das Südfeuer mit $» \mathrm{Du}$ sollst glänzen! ¿23 $^{23}$

20. Das Besprengen ist (bereits) erwähnt ${ }^{224}$ worden.

21. Zu diesen beiden (Besprengungen rechnet man auch) das zweimalige Auffegen (der Opferstätte).

22. (Dieses) aber erfolgt vor dem Besprengen.

23. $\mathrm{So}^{225}$ ist es auch morgens ${ }^{22 \theta}$.

24. (Man opfert) gegen Sonnenaufgang, während der Morgendämmerung oder nach Sonnenaufgang 22 .

${ }^{213}$ Vgl. Ap VI, 12, 4; Käty IV, 14, 28.

214 Vgl. TS IV, 1, 9, 1; MS II, 7, 7; KS XVI, 7; VS XI, 66; AB VII, 9, 1 ; SB

VI, 6, 1, 20; Käty XXV, 12, 10; SānkhGṛs V, 4, 2.

$215 \mathrm{Vgl}$, Mân $\mathrm{I}, 6,1,50$.

216 Nur an dieser Stelle.

217 Konm. und Pray.

218 Komm, : auf den ahavaniza, Pray. läBt einige Lehrer sich für den gärhapatya entscheiden.

${ }^{210}$ Komm. und Pray.: nach Silden.

220 D. h. drei in jedes der drel Feuer.

$221 \mathrm{Vgl}$. Ap VI, 13, 10-11.

222 S. Anm. 221.

223 Nur hler.

224 S. II, 2, 11.

225 Nämlich wie betm socben geschilderten Abend-agnihotra.

228 Jedoch unter Beritcksichtigung der folgenden Besonderheiten.

227 Ass vereinigt hier verschicdene sich sonst bekämpfende Standpunkte (vgl.

A. Weber, Indische Studien, X, S. 329); freflich ist efne derartige Zeitbestimmung

8 Acta Orientalla, XXXIV 


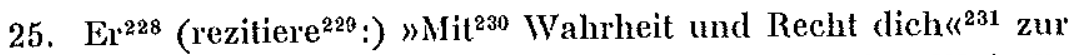
Besprengung; mit $\gg \mathrm{Om}$, ich werde herausschöpfen « ${ }^{232}$ (vollzieht er) die Entsendung; mit "Als golden, somnenleuchtend, lege ich dich, den Tag, als Ziegel an, svähal ${ }^{233}$ (vollzieht er) die Brennholzanlegung, und mit "Bhür bhuval svar om « ${ }^{234}$, "Sürya ist das Licht, das Licht ist Sürya, svähăl« ${ }^{235}$ (erfolgen) die Opferung und das Abwischen ${ }^{236}$.

Ende der 4. kaṇḍikā

II, 5

1. Jemand, der zu verreisen beabsichtigt ${ }^{237}$, soll, nachdem er die Feuer entflammt und (den Mund) ausgespüll hat und entlang geschritten ist ${ }^{238}$, verehren -

2. den ähavañy mit »O Preisenswerter, sehülze mein Vieh! ${ }^{239}$; den gärhapatya mit »O Mannhafter ${ }^{240}$, schütze meine Nachkommenl«241; das Südfeuer mit »O Atharva ${ }^{242}$, schütze meine Nahrung! $\ll^{243}$. Er schaue auf gärhapatya und ăha-

fiir einen hotar, an den sich das AśśrS ja vorzugsweise wendet, von untergeordneter Bedeutung.

2 8 Der Priester.

22. Es folgen die Unterschiede in der Arbeltsweise des Priesters im Vergleicl zum abendlichen agnihotra.

2so Vgl. II, 2, 11.

231 Vgl. Mān I, 6, 1, 10.

232 Dies nur hier; vgl, aber II, 3, 10.

233 Vgl. zun mantra Ap VI, 9, 4; zum Inhalt Ass II, 3, 15.

$234 \mathrm{Vgl}$. Anm. 161 zu II, 3, 16. Das Om ohne pluti.

29s Vgl. SV II, 1181; MS I, 6, 10; II, 7, 16; KS VI, 5; XL, 6; VS III, 9; AB

$\mathrm{V}, 31,4$; KB II, 8 ; XIV, 1; ȘB I, 4, 9; TB II, 1, 9, 2; SB II, 3, 1, 30.33.36; TA

IV, 10,5; V, 8, 10; Śānkh II, 9, 2; Lăṭy I, 8, 14; Ap VI, 10, 8; XV, 12, 8; XVI, 23,10 .

2s 6 Vgl. II, 3, 16.20. - Hiermit endet die Schllderung des morgendlichen agnihotra.

237 Und wenigstens eine Nacht fortblebt (Komm.).

238 An dem jeweils verehrten Feuer (Pray.).

239 Vgl. VS III, 37; TB I, 1, 10, 2.4. 2, 1, 25; SB II, 4, 1, 5; Sãnkh II, 14, 4;

Äp V, 18, 2.

240 Statt naya ist narya zu lesen.

241 S. Anm, 239.

242 VS und Sānkh lesen atharya.

${ }_{243} V_{g l}$ TB und $\bar{A}$ p wie in Anm, 239. 
vanīya (mil:) "Diese meine Häuser, o Mitra und Varuna ${ }^{244}$, behütet ${ }^{245}$ Als unversehrte, ungeöllinete ${ }^{246}$ soll Püșan sie beschützen ${ }^{247}$ bis zu unserer Rückkehr. ${ }^{248}$

3. Nachdem er auf dem gleichen $W^{2}{ }^{249}$ zurückgekehrt ist, verehrt er nach Süden gewandt den ähavaniya mil: "Meinen ersten Namen, o Jätavedas ${ }^{250}$, den Vater und Mutter (mir) zu Anfang gaben ${ }^{251}$, den trage du bis zu meiner Wiederkehr $^{252}$; deinen Namen will ich tragen zu Anfang ${ }^{253} \cdot{ }^{254}$

4. (Nun) schreite er hinweg, ohne sich umzusehen, wobei er die Hymne $»$ Nicht wollen wir verlassen ${ }^{255} 256$ flüstert.

5. Fern von den Feuern ${ }^{257}$ erhebe er (wieder) die Stimme.

6. Mit "Immer gut begehbar, nahrungsreich ${ }^{258}$ soll der Weg sein ${ }^{259}$ betrete er den Weg.

7. Wenn er, ohne daß das Feuer (vorher) verehrt ${ }^{260}$ wurde, eine Reise antreten muß ${ }^{261}$, dann verehre er mit $\gg$ Hier mich befindend, erhalte ich dich, den sich dort befindenden, o

244 Vgl, MS I, 5, 14; KS VII, 3.11; Ás II, 5, 12; Ap VI, 24, 4; Màn I, 6, 3, 8.15; Sāǹnkigṛ III, 6, 2.

245 Wie Amm. 244, doch ohne Ăśv II, 5, 12 und Mān I, 6, 3, 15.

2s6 Vgl. KS VII, 3; MS, Asv, Ap, SānkhGṛS wie in Anm. 244.

247 Vgl, MS, KS, $\Lambda$ p, SānkhGṛs wie in Amm. 246.

248 Män I, 6, 3, 7; MS, KS, $\AA_{p}$, SānkhG̣̣S wie in Amm. 246; ferner Ảśv II, 5, 12.

218 Der Weg ist nach dem Komm. kurz folgender: ähavaniya, gärhapalya, Sildfeuer; von dort Blick auf ahavaniya und gärhapatya; Rückkehr auf demselben Weg zum ahavaniya, wo die obigen mantras gesprochen werden.

250 Vgl. TS I, 5, 10, 1; KS VII, 3.11; $\AA_{p}$ VI, 24, 7; Māı I, 6, 3, 9; HirGṛs II, 4,11 .

251 Vgl, TS und Mān aus Anm. 250.

$2 s 2$ S. Anm. 251.

${ }^{253}$ Die Parallelstellen haben sinnvoller agne no Agni".

254 Vgl. TS I, 5, 10, 1.

${ }^{255}$ Statt pranameti, das auch der Komm. fälschlich hat, ist mit der pray. pragameti zu lesen.

256 pratika aus má pra gäma patho vayam RV $\mathrm{X}, 57,1$; vgl. $X \delta \mathrm{V}$ II, 19, 36; VI, 6,18 .

257 Komm.: sobald man das Dach der Hüte, in der die Feuer untergebracht sind, nicht mehr sehen kann,

${ }^{258}$ Statt plturmä ist pitumǟ zu lesen.

259 R.V III, 54, 21.

200 Statt anapasthita ist anupasthita zu lesen.

261 äpadyeta. 
Agni $^{262}$, durch Herz, Sprache oder Geist. ${ }^{263} \mathrm{Mich}$, der ich abseits ${ }^{264}$ bin, verlasse nicht $\left.\right|^{265}$ Mit dem Licht, mit Vaiśvānara verehre ich ${ }^{260}$ dich ${ }^{207}$ die (einzelnen) Feuer in ihrer (jeweiligen) Himmelsrichtung. ${ }^{268}$

8. „Den Weg betraten wir ${ }^{269}$ (reziliere er), nachdem er zurückgekehrt ist.

9. Mit Brennholy in der Hand und nichts Weltliches redend ${ }^{270}$ fachen die beiden ${ }^{271}$ die Feuer an, nachdem sie (sie) erkannt $^{272}$ haben. Herangetreten, blickt er auf den ähavanìya (und spricht:) $/$ Immerdar herbcitragend ${ }^{273}$ mit gesundem Geiste ${ }^{274}$, o Agni, nicht mögen wir, dir benachbart, zu Schaden kommen!275 Verehrung sei dir, dem Freigebigen ${ }^{276}$; Verehrung dir, dem Huldigung ist ${ }^{277}$; o Agni, schmïcke den Körper ${ }^{278}$, vereinige mich mit Reichtum $\mid \ll^{279}$

10. Nachdem er Brennholzscheite in die (einzelnen) Feuer niedergelegt hat, verehrt er den ähavanüya (mit ${ }^{280}$ :) »Meinen Namen und deinen, o Jătavedas ${ }^{281}$, die wir beide ${ }^{282}$ wie zwei Ge-

\footnotetext{
263 Vgl. TB II, 5, 8, 7; Mān I, 6, 3, 18.

263 Vgl, Mān aus Aum, 262.

204 D. h. entfernt von den häuslichen Opferfenern.

265 Vgl, mit Varianten TB I, 2, 1, 27; II, 5, 8, 7; $\AA_{p}$ VI, 25, 2.

${ }^{268} \mathrm{Stat}$ upatişhate ist wohl besser upalisthe zu lesen.

267 Vgl, TB II, 5, 8, 8 .

${ }^{268}$ Nach der Pray, Im Osten den ahavaniya, im Westen den gärhapalya, im Sütuen den dakșinăgni.

269 Vgl. RV VI, 51, 16.

270 väguata.

271 Komm.: der ähilägni mit einem Sohn oder Schülex.

272 śrutva, Komm.: jnatuă. Pray, bietet keme Erklärumg.

273 Vgl. $\lambda_{p}$ VI, 2, 2. 25, 7; Mān I, 6, 3, 12.

274 S. Anm, 273.

275 Vgl. TS IV, 1, 10, 1; MS II, 7, 7; KS XVI, 7; VS XI, 75; SB VI, 6, 3, 8; Ap und Man wie in Anm. 273.

276 Vgl. Ap VI, 25, 7.

271 S. Anm. 276.

278 S. Anm, 276.

270 S. Amm. 276.

280 Bezieht sich auf $I I, 5,3$.

a81 Vgl. T'S I, 5, 10, 1; Ãp VI, 26, 4.

2sa Die Vedic Concordance hat Irrig căraval statt carāvah; erwarten sollte man frellich besser bharävah.
} 
wänder tauschend tragen ${ }^{283}$, die tragen wir beide zu Kraft und Leben ${ }^{284}$, demgemäß unsere Körper ${ }^{285}$, o Jātavedas. « ${ }^{280}$

11. Darauf lege er Holzscheite an -

12. in den ăhavanūya (mit:) "Gegangen sind wir zum Allbesitzenden $^{287}$, zu dem uns am meisten Reichtum Verschaffenden ${ }^{288}$. O Agni, Oberkönig, Herrlichkeit ${ }^{269}$, Stärke verleihe! !20 $^{290}$ Svähäl« - In den gärhapatya (mit:) »Dieser Agni als Hausvater $^{291}$ ist der Herr über das Haus, der der Nachkommenschaft am meisten Reichtum Verleihende ${ }^{292}$. O Agni, Hausvater, Herrlichkeit ${ }^{293}$, Stärke verleihe! ${ }^{294}$ - In das Südfeuer (mit:) $)$ Dieser Agni ist reich an Land ${ }^{205}$, wohlhabend, Gedeihen fördernd ${ }^{290}$. O Agni, reich an Land ${ }^{297}$, Herrlichkeit, Stärke verleihel«298 $-\mathrm{Er}$ schaue auf gärhapatya und ăhavañya (mit:) »Diese meine Häuser, o Mitra und Varuṇa ${ }^{299}$, habt ihr behïtet ${ }^{300}$. Als unversehrte, ungeöllinete ${ }^{301}$ hat Püșan sie beschützt ${ }^{302}$ bis zu unserer Rückkehr ${ }^{303}{ }^{304}$

283 Vgl. 'T'S I, 5, 10, 1; IXS VII, 3; Mān I, 6, 3, 66.

28 Vgl, ISS VII, 3; Mân I, 6, 3, 16.

285 Es ist besser mit KS tanvau zu lesen,

286 S. Anm. 284.

287 In dieser Form nur hier.

288 Vgl. VS III, 38; SB II, 4, 1, 8; Sānkh II, 15, 2.

282 S. Anm, 288.

200 Vgl, VS III, 38-39; SB II, 4, 1, 8; Sănikh II, 15, 2.4-5.

201 Vgl. VS III, 39; SB II, 4, 1, 9; Sănkh II, 15, 5; ĀpGṛS II, 6, 10

292 Vgl. VS III, 39; SB II, 4, 1, 9.

${ }^{203}$ S. Amm. 292 und Säńkh 1I, 15, 5.

284 Identisch mil dem mantra unter Anm. 290.

295 Vgl, VS III, 40; Sănkh II, 15, 4.

296 S. Anm, 295.

201 S. Anm, 295.

298 S. Anm. 294.

299 S. Anm, 244 zu II, 5, 2.

$300 \mathrm{Vgl}$. allenfalls $\lambda_{\mathrm{p}}$ VI, 26, 3 .

s01 S. Anm, 240 zu II, 5, 2 ,

302 Vgl. MS I, 5, 14.

303 S. Anm, 248 zu II, $5,2$.

sos Da $\Lambda$ śvalāyana hier nicht einen Priester, sondern einen Laien instruiert, ist fur thn die Heranziehung von mantras aus den RV nicht erforderlich. So entnimmt er die mantras ziemlich ekfektisch bald aus tem Schwarzen, bald - wie im vorstehenden sîtra - aus dem Weißen Yajurveda. 
13. Nachdem er (auf dem IVege), wie gekommen, zurückgekehrt ist, aufgefegt ${ }^{305}$ und sich nördlich vom Feuerplatz niedergelassen hat, soll er mit "bhür bhtwah svah « ${ }^{300}$ die Stimme erheben.

14. Wenn er länger als zehn Nächte fortgewesen ist, bringe er eine vierfache Opferschmalzspende dar (mit:) $»$ Der Geist freue sich am Lichte, an meinem Opferschmalz, ${ }^{307} \mathrm{Er}$ soll dies zerrissene Opfer (wieder) zusammenfügen ${ }^{308}$. Die beopferten Morgenröten wie die nicht boopferten ${ }^{309}$, die füge ich zusammen mit Opfergabe, mit Schmelzbutter ${ }^{310}$. Svăhă!

15. (Dies gilt) auch bei einer Nichtdarbringung des agnihotr $a^{311}$.

16. Einige (lehren:) pratihomam ${ }^{312}$.

17. Auch (ein Zurückkehrender), der nicht dic heiligen Feuer angelegt hat, soll auf (seine) Wohnstätten blicken (und zwar mit:) "Häuser, fürchtet euch nicht ${ }^{313}$, der Trefflichste euch zum Heil ${ }^{314}$, pflanzt euch in uns fort. Nicht möge euch der Gebieter ${ }^{315}$ schädigen. « Er trete ein (mit:) »Dic wohlgesinnten Häuser betrete ich ${ }^{316}$, nicht Männer tötend, die Männer besitzenden, gute Männer besitzenden. ${ }^{317}$ In die Erquickung

305 ISomm.: die Ungebung der Feuerstätten.

${ }^{308}$ S. Anm. 186 zu II, 3, 27.

${ }^{307}$ Vgl. TS I, 5, 3, 2. 10, 2. 6, 3, 3; MS I, 7, 1; IV, 8, 9; KS XXXIV, 19; VS II, 13; VSK II, 3, 11; TB III, 7, 6, 16; SB I, 7, 4, 22; Läty IV, 12, 1; Ap III, 3,$2 ; \mathrm{V}, 27,13$; VI, 26, 7; IX, 8, 1. 13, 8. 17, 2; XIV, 16, 1. 17, 1, 28, 2; Kāty XXV, 10, 22; Vait IV, 3 .

308 Vgl, TS I, 5, 3, 2, 4, 3. 10, 2. 6, 3, 3. 7, 1, 5; MS I, 7, 1; IV, 8, 9; TB III, $7,6,16 ;$ Käty XXV, 10, 22.

${ }^{300}$ Vgl. MS I, 7, 1; Käty XXV, 10, 22.

s10 Vgl. Käty $\mathrm{XXV}, 10,2$. Die Spruiche dieses sūtra haben eine Anzalı von Varianten.

an $\mathrm{D}$. h. wenn die Reihe der agnihotra-Opfer eimmal aus irgendeinem Grunde, wie etwa auf der Reise, unterbrochen wurde.

s12 Nach dem Komm. bedeutet dies, man solle so viele Opfer darbringen, wie sie der Zeit der Unterbrechung entsprechen; es ist hier also an ein Nachholen jedes einzelnen Opfers gedacht.

sıз $\AA_{p}$ VI, 27, 3a. Von hier an ist der Spruch ohne Parallele in der vedischen Literatur.

314 Die Stelle ist jedenfalls verderbt; statt svastye ist wohl svastaye zu lesen.

315 Dfeser mantra-Tell ist vermutlich eine spätere Interpolation; evtl. ist mit gopali (hier neutral mit „Gebieter übersetzt) bereits Kṛș̣a gemeint.

ง16 Vgl, Åp XVI, 16, 4; HirGṛS I, 29, 2; MānGṛS I, 14, 6.

317 Vgl. mit Varianten SănkhGṛS III, 5, 3; Ap und Hir wie in Anm. 316. 
bringenden, Schmelzbutter träufelnden ${ }^{318}$ (Häuser) will ${ }^{319}$ ich wohlgemut eintreten ${ }^{320}$, indem er mit "Freundlich, gütig, Glück und Segen, Glück und Segen« ${ }^{321}$ dreimal sich umschaut.

18. Eine bekannt gewordene Unammehmlichkeit soll man (dem Heimkehrenden) an diesem Tage nicht mitteilen ${ }^{322}$.

19. Bekannt ist, (daB) man (mit dem Spruch) "Sicherheit euch, Sicherheit sei mirl«323 verehren soll, wenn man verreist, wenn man zur'icckgekehrt ist oder ${ }^{324}$ Tag für Tag ${ }^{325}$.

Endle der 5. kaṇdikā

II, 6

1. Am Neumondstage nachmittags (erfolgt der) piṇlapilryajña ${ }^{326}$.

2. (Der Opferveranstalter entnehme) aus dem Südfeuer einen Feuerbrand (und) trage ihn nach Südosten (wobei er spricht:) "Die (verschiedene) Gestalten Annehmenden ${ }^{327}$, die, Dämonen seiend, nach Belieben wandeln ${ }^{328}$, außerhalb und innerhalb der Siedlungen sind ${ }^{329}$, die soll Agni von dieser Welt hinwegtreiben. $\ll^{330}$

318 Vgl. Ap VI, 27, 5; ÃśvGṛS II, 9, 5; ŚānkhGṛS III, 5, 3; MānGṛS I, 14, 6; II, 11, 17.

${ }^{3 \times 8}$ Es ist samoisâni statt samísáali zu lesen.

320 Vgl. Āp VI, 27, 5; HirGṛS I, 29, 2; Mān wie Anm. 318.

s21 Ohne Parallele.

322 Ein psychologiseh treffender Ratschlag!

323 Vgl. AB VII, 12, 8; Sảnkh II, 14, 1 ff.

${ }^{\text {s24 }}$ Nach dem Komm. soll noder hier für sund stehen.

${ }^{325}$ Hiermit schlient die Darstellung des agnihotra.

326 Ein an die Manen gerichtetes Opfer mit Mehlklößen; $s$, die Monographie von $O$. Donner: Pindaplțyajna, das manenopfer mit klössen bei den Indern, Berlin 1870; besonders S. 28-29.

927 Vgl. VS II, 30; ŚB II, 4, 2, 15; Sãnkh IV, 4, 2; Āp I, 8, 7; Kauś LXXXVIII, 1; HB II, 3, 4; GobhG IVS 3,3 .

${ }^{928} \mathrm{~S}$. Amm. 327 außer Gobl..

929 Vgl. Anm. 328 sowie AV XVIII, 2, 28; Mān I, 1, 2, 8. Die Stelle ist nur versuchsweise übersetzl unter Änderung von bharanti in bhavanti. Bereits die Kommentatoren haben die Passage nicht mehr verstanden; so erklärt der Komm. zu VS II, 30 paräpuras mil sthäladehän, nipuras mit sükşmadehän (ovon grober bzw. zarler Gestalt»); der Komm. zu MB liest parā puro ni ptro und erklärt: ndie Häuser unserer Feinde bzw. Verwandtene.

${ }^{330}$ S. Aum. 27 auBer Kauś und Gobh. 
3. Alle (folgenden) Riten (erfolgen) in diese Himmelsrichtung ${ }^{331}$ (gewandt).

4. Nachdem er auf beide ${ }^{332}$ (Feuerungsmaterial) nachgelegt (und die Opferstreu) ringsum ausgestreut hat, setze er nor(östlich oder nordwestlich rom Südfeuer einzeln (folgende) $O_{\mathrm{p} f e r g e r a ̈ t e}{ }^{333}$ hin: einen Tonkochtopf $\mathrm{f}^{334}$ für Reisbrei ${ }^{335}$, eine Getreideschwinge ${ }^{336}$, ein Holzmesser ${ }^{337}$, einen Mörser ${ }^{338}$, cinen Stößel ${ }^{339}$, einen kleinen Opferlöffel ${ }^{340}$, einen dhruvãLöffel $^{341}$, ein schwarzes Antilopenfell ${ }^{342}$, mit éinem $\mathrm{Mal}^{343}$

331 Südosten.

332 Nämlich auf das Südfeter und das daraus entnommene Feuer.

งэз Eigentlich pätra Gefäße.

${ }^{33}$ sthâli; vgl, TS VI, $5,10,2-3$; ISS VI, 3; KKS IV, 2; MS I, 8, 3.6; VS XIX, 27.86; VSK XXI, 2, 16; AB I, 11, 8; JB I, 54 ; TB II, 1, 3, 1; SB III, 2, 3, 21-22; IV $2,2,1.16$; VI, $6,4,8 ; \mathrm{XI}, 3,2,1$; SBK $1,5,1,38 ; \bar{A}_{\mathrm{p}} 1,8,1$.

${ }_{335}$ caru, Mus oder Suppe von Reiskörnern und Wasser mit Mileh und Butter; vgl. TS I, 8, 10, 1; AB I, 1.7; JB I, 167; SB I, 7, 4, 7; II, 5, 3, 4; III, 2, 3, 1; IV, $4,2,1 ; V, 1,4,2.2,4,11 ;$ VI, $6,1,8$.

sas sürpa, aus Bambus-Korbgeflecht bestehend; vgl. TS I, 6, 8, 3; KS XXXVI, 6; MS $1,10,11$; VSK II, 5, 2; SVB III, 4, 2; TB I, 6, 5, 4; III, 2, 5, 11. 7, 6, 21; SB I, 1, 22. 4, 19-20; II, 5, 2, 23; SBK II, 1, 2, 1; Ap I, 7, 5. 17, 10; Kanty 1, 3, $36 ;$ II, 3,8 .

${ }^{337}$ sphya, gewöhmlich aus dem Holz von Acacia catechu, diente u. a. zur Markierung der Unrisse der Opferstätle (vedi), so SB III, 3, 1, 5; vgl. ferner 'IS I, $6,8,2 ; \mathrm{II}, 1,8,2$; VI, 6, 4, 1; KS XX, 4; KKS XXXI, 6; MS II, 1, 9; AB VIII, 5 ; TB I, 7, 10, 5; JB I, 48; SB I, 1, 1, 22. 2, 4, 2-3. 5, 20; III, 6, 2, 12; V, 4, 4, 15.20; SBK II, 2, 2, 1; $\lambda_{\mathrm{p} \mathrm{I}}$ I, 8, 8. 25, 14; II, 2, 4; III, 8, 5; Käty I, 3, 33.39.

${ }_{938}$ ulükhala; vgl. TS I, $6,8,3 ; \mathrm{V}, 2,8,7,6,9,2 ; \mathrm{VII}, 2,1,3 ; \mathrm{KS} \mathrm{XX}, 7$; KKS XXXI, 9 ; MS II, 7, 16; III, 2, 7; VSK II, 5, 2; JB I, 48; II, 298; TB III, 7, 6, 21; SB I, 1, 1, 22. 4, 6-8; II, 2, 2, 1; IV, 3, 4, 1; VI, 1, 2, 30; VII, 5, 1, 12.16. $22.25-27.38 ; \mathrm{X}, 4,3,14.5,5,7$; XI, 1, 2, 1; XII, 5, 2, 7; SBB II, 1, 2, 1. 3, 12. 4, 19. 2, 1, 8; Sănikh IV , 3, 2; Ap XVI, 26, 1; Kăty I, 3, 36.

${ }^{339}$ musala; vgl. TS I, 6, 8, 3; VSI II, 5, 2; JB I, 42.44; III, 221; TB III, 7, 6, 21. $8,4,1$; \$B I, 1, 1, 22. 4, 10; VII, 5, 1, 38; XII, 5, 2, 7; SBK II, 1, 3, 14 ; SA XII, 8; Kăty I, 3, 36 .

3н sruva; vgl. TS III, 5, 7, 1; KS VI, 8; KKS IV, 7; MS I, 11, 8; AB II, 12; SVB II, 5, 3; III, 6, 12; JB I, 40; TB I, 5, 9, 5; III, 2, 9, 14; SB I, 3, 1, 9.14. 9, 2, 1.16; II, 5, 3, 6; V, 2, 2, 4. 4, 15; XIII, $8,4,8$; SBK I, 5, 2, 5.10; GB I, 3, 11-12; AA V, 1, 1; SA IV, 3; XI, 4; JUB III, 17, 2; BU VI, 3, 21; Äp I, 15, 10; Kăty I, $3,32.36 .38$.

${ }^{341}$ Ein Opferlöffel aus vikankala-Holz; vgl. TS I, 7, 5, 1; III, 5, 7, 3; SB I, $3,2,2.8,3,13 ; \Lambda_{\mathrm{p}}$ I, 15, 10; VIII, 10, 4; Sānkh V, 8, 2.

s4a krṣäjina; vgl. TS II, 4, 9, 2; V, 4, 4, 4; AB I, 3.13.30; VII, 23; KB IV, 
abgeschnittenes kuśa-Gras ${ }^{344}$, Bremnholz, einen Rührlöffel ${ }^{345}$ und einen Wasserkrug ${ }^{346}$.

5. Nachdem er von Süden her den agniṣlha (genamnten Wagen $^{347}$ ) bestiegen hat, fülle ${ }^{348}$ er den Reisbrei-Tonkochtopf voll mit Reiskörnern.

6. Die hinübergefallenen ${ }^{340}$ (Körner) lege er nieder ${ }^{350}$.

7. Nachdem der Mörser aur das Antilopenfell gestellt wurde, soll die Gattin ${ }^{351}$ (lie anderen ${ }^{352}$ (Körncr) dreschen, ohne sie gesiebt zu haben.

8. Nachdem er die gedrosehenen (Körner) einmal gewaschen hat, lasse er sie auf dem Südfeuer kochen.

9. Zwischen ${ }^{353}$ (dem Südfeuer und) dem herangetragenen ziehe er mit dem Holzmesser ${ }^{354}$ eine Linie (und spreche:) $)$ Hinweggeschlagen sind die Dämonen, die auf der Opferstätte sitzenden Unholde. ( $^{355}$

10. Nachdem er diese (Linie) besprengt und mit den mit einem (Hieb) geschnittenen (Gräsern) bestrent hat, tue er zum Fetträufeln in das (in der) sthäl ${ }^{350}$ gekochte (Reisgericht) Schmalz, ungereinigte zerlassene Butter oder gercinigte

11; РВ XVII, 11, 8; XXV, 14, 2; JB II, 67; 'TB II, 7, 1, 4. 3, 3; ŚB I, 1, 1, 22 .

$4,1,9.2,35$; VI, 2, 2, 39. 4, 1, 6. 7, 1, 6; XIV, 3, 1, 21 ; SBI II, 1, 2, 1. 3, 8.10.12,

4, 12.14; GB I, 3, 21; JUB III, 8, 7; TA V, 2, 11.13; Аp X, 8, 11 .

313 D. h, mit éinem Hiel.

ant So der IKomm.

sts mekşąa; ist aus Holz, hat die Lïnge einer Elle und besitzt vier Greifzähne; vgl. KS XXIII, 9; XXIX, 2; IKKS XXXVI, 6; XLV, 3; MS III, 7, 2; JB I, 60; TB I, 3, 10, 4; III, 7, 4, 9 ; SB II, 4, 2, 13; III, 2, 3, 21-22; XII, 1, 2, 1 ; ŚBIK I, 3, 3, 11; Ap I,7,5; XIII, 13, 17.

${ }^{316}$ kamandalu; vgl. Åp III, 8, 1.

as? Komm, und Pray.

348 nimpjel, eigentl. øreibe hinabø; nach Komm. und Pray. dadurch zu erklären, daB ein súrpa (s. Anm. 336) benutzt wird.

sı9 Komm.: uber den Rand des sürpa.

${ }^{350}$ Komm.: auf den Wagen, d. h. er lasse sie dort liegen.

351 Des Opferveranstalters.

${ }^{358}$ Konm.: die in der sthäl (s. Anm. 334) enthaltenen.

353 Nach dem Komm.

354 S. Anm. 337 zu II, 6, 4.

355 Vgl. VS II, 29; MB II, 3, 3; Șañkh IV, 4, 2; Ãp I, 7, 13.

${ }^{856}$ S. Anm. 334 zul II, 6, 4. 
(frische) Butter; das Schmalz hat er (zuvor) auf die dhruba $\bar{a}^{357}$ sïdlich (des Südfeuers ${ }^{358}$ ) getan.

11. Dorthin ${ }^{359}$ (bringt er auch) Augensalbe, Salbe, Matte und Kissen.

12. Angetan mit der heiligen Schnur ${ }^{360}$ opfere er, nachdem er Brennholz aufgelegt ${ }^{361}$ und den Rührlöffel ${ }^{362}$ herausgenom$m^{36 n^{363}}$ hat, mit der Handlung des Austeilens (der Portionen, indem er sagt ${ }^{364}$ :) $)$ Dem Soma mit den Ahnen svadhă (und) Verehrung $\ll^{365}$ und $»$ Den Agni, dem Manenopferfahrer svadhã (und) Verehrung ${ }^{366}$.

13. Oder (er opfert) mit dem Svăhă-Ruf, (dann aber) zuerst an Agni $^{367}$, angetan mit der Opferschmur ${ }^{368}$.

14. Nachdem er den Rührlöffel dargebracht hat, angetan mit der heiligen Schnur (über der linken Schulter), begieße er die Linie ${ }^{369}$ (dreimal mit Wasser (und spreche:) »Reinigen sollen sich die Väter ${ }^{370}$; reinigen sollen sich die Großväter ${ }^{371}$; reinigen sollen sich die Urgroßväter. $\ll^{372}$

15. Auf diese (Linie) schülte er die Reismehlklösse mit nach unten gerichtetem Handrücken für den Vater, den Groß-

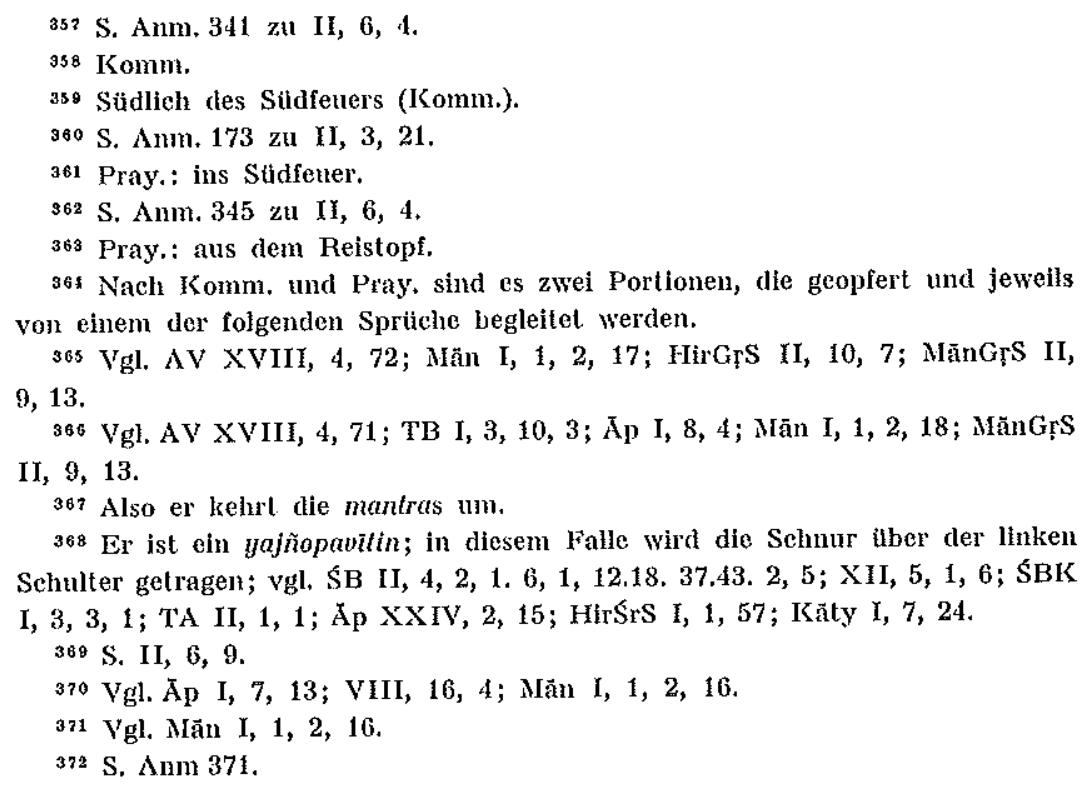


vater und den Uigroßvater aus (und spreche:) »Dies für dich, N.N., und die, die dir nach (-folgen). $«^{373}$

16. (So verhalte er sich) gegenüber denjenigen von ihnen, dic verstorben sind, (sagl) Gạnagāri; direkt chre man die ande$r^{1}{ }^{374}$ wegen des Sinns dieses (Ritus $\left.{ }^{375}\right)^{376}$.

17. Fïr alle (Vorfahren ${ }^{377}$ ) soll er aussehütten, (sagt) 'Taulvali, wegen der Bedeutung (dieser) Zeremonie.

18. Vom (letzten) Lebenden $a^{378}$ soll er für drei Verstorbene ${ }^{379}$ ausschütten, (sagt) Gautama; der Ritus ist nämlich zweck. bedingt.

19. (Es ${ }^{380}$ besteht) ein Unterschied der Mittel für die (noch) lebenden und die toten (Ahnen).

20. Nicht den entfernten (Ahnen), weil sie keine Funktion mehr ausüben $^{381}$; nicht den anwesenden, nicht den lebenden (schütte man aus).

21. (Auch) nicht für die durch einen Lebenden getrennten (Alinen) ${ }^{382}$.

22. Man opfere ${ }^{383}$ den Lebenden.

23. Alle (drei pindlas) werden geopfert ${ }^{384}$, wenn alle (drei Vorfahren noch) leben.

24. Wenn man die Namen (der Vorfahren) nicht kennt, (sage man algemein:) "Vater, Großvater, Urgroßvater."

Ende der 6. kaụdika

s73 In dieser Form nur hier.

374 D. h. die noch lebenden Vorfahren.

37s Der nach dem Komm. darin besteht, dle Ahnen zu sättigen.

370 Mit diesem sūtra beginnt eine Polemik des Åsvalăyana gegen dic Meinungen dreier anderer Lehrer.

an7 Komm.: den lebenden wie den toten.

${ }^{378}$ Etwas fret und nach dem Komm, iblsersetzt.

379 D. J. die jewefligen direkten Vorfahren in drei Generationen,

s80 Asvalāyana beginnt jetzt, sich mit den Ansichten seiner Gegner auseinanderzusetzen.

${ }^{381}$ adhikära (Komm, ayogyatva) kann doch nicht gut mit \$Abteilung übersetzt werden, wie es bei Dommer geschicht.

${ }^{382}$ Also eine Zurickweisung des Gautama; s. sütra 18.

${ }^{383}$ Dies ist keh Widerspruch, dem hier heiBt es juhtyăl, während es von der den Ahmen zukommenden pinda-Spende stets heißt: nipraīyät. Bei einem homa aber handelt es sich gewöhnlich um eine Opferung ins Feuer.

384 sarvahutam, also hiennach und nach den sütras 20 und 22 ins Fener. 
II, 7

1. Die ausgeschütteten (Reismehlklösse) rede er an mil: "Hicr, o Ahnen, ergölzl euch, jeder zu seinem Anteil wie Stiere kommet $\mid$ « $^{385}$

2. Nach links gewendet, wendet er sich nach Norden ${ }^{386}$. Nachdem er nach Kräften den Atem zurückgehalten hat ${ }^{387}$, wendet er sich wieder (den Klössen) zu (und spricht:) $\gg$ Firgötzt haben sich die Ahnen, jeder zu seinem Anteil wie Stiere sind sie gekommen ${ }^{388}, \ll^{389}$

3. Vom Reisbrei soll er einen Atemverzehr genießen ${ }^{330}$.

4. Obligatorisch ist (jetzt wieder) das (Wasser-) Ausgießen ${ }^{391}$.

5. (Nit) $» N . N$. , salbe dich; N.N., salbe dir die Augen $/ \ll^{392}$ (streiche er) auf die Reismehlklösse Salbe und Augensalbe.

6. Zur Bekleidung (der Ahnen) gebe er (auf die pindlas ${ }^{393}$ ) einen Kleidersaum oder Wollbüschel; wenn er über 50 Jahre (alt) ist, eigenes Haar (mit:) »Dies, o Ahnen, (sei) euer Gewand ${ }^{394}$; zieht nichts anderes, o Ahnen, als das von uns $\operatorname{an}^{395} \mid \ll^{396}$

7. Nun verehre er sie ${ }^{397}$ : $»$ Verehrung, o Ahnen, eurem Saft ${ }^{398}$; Verehrung, o Ahnen, eurer Kraft ${ }^{399}$; Verehrung, o Ahnen,

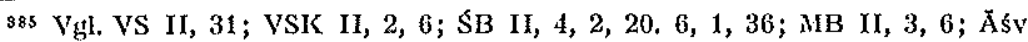
V, 17, 5; Śñnkh IV, 4, 11. 9, 2; VIII, 2, 13; Lāṭy II, 10, 4; Mān II, 5, 1, 35; Kauś LXXXVIII, 18; GobhG̣̣S IV, 3, 11; KhädGịS $I I I, 5,18$; ferner diverse Varianten.

386 Urspringlich blickte er It. II, 6, 3 nach Südosten.

${ }^{387}$ Der Text hat hier eine falsche Vorttrennung; es muß yathäsaklyapränannăsilvä heißen.

as Vgl. VS II, 31; VSK II, 2, 6; SB II, 4, 2, 22, 6, 1, 40; MB II, 3, 7; Śăìlkh IV, 4, 14. 9, 3; Lāty II, 10, 5; Kauś LXXXVIII, 21; GoblıG̣̣S IV, 3, 12; KhādGṛS III, $5,20$.

380 Es galt also als unschicklich, den Almen beim Essen zuzuschauen.

390 D. h. er soll daran mur riechen.

a91 Der Ritus nach dem Komm. wie oben (II, 6, 14),

302 In dieser Form nur hicr.

ง93 Komin.

394 Vigl, VS II, 32; VSK II, 7, 4; MB II, 3, 14.

305 In dieser Form nur hier.

396 Damit galten die Bedürfnisse der Ahnen als befriedigt.

ao Pray.: die pindas,

398 Vgl. MS I, 10, 3.

393 Vgl. AV XVIII, 4, 81; MS I, 10, 3. 
eurem Ungestüm ${ }^{400}$; Verehrung, o Ahnen, eurem Schrekken ${ }^{401}$; Verehrung, o Ahnen, eurem Leben ${ }^{402}$; Verehrung, o Ahnen, eurer Leidenschaft ${ }^{403}$; svadhä euch, o Ahnen ${ }^{40 ;}$; Verehrung etch, o Ahnen, Verehrung ${ }^{405}$. Jene gehören euch, o Ahnen, diese uns ${ }^{408}$. Als Lebewesen ${ }^{407}$ möchten wir hier als Lebende existieren ${ }^{408}$,

8. Und (er l'ezitiere) die (mit) »Den Geist herbei wir rufen «409 (beginnenden) drei (Strophen).

9. Dann sendet er $\operatorname{sie}^{410}$ weg (mit:) „Gehet hin, ihr lieben Ahnen $^{411}$, auf den tiefen Pfaden, den alten ${ }^{412}$. Uns Wohlhabenheit und Glück hier gebend ${ }^{413}$, gewährt uns Reichtum für alle Männer|«\$14

10. Dem Feuer ${ }^{415}$ gehe er entgegen (mit:) $» \mathrm{O}$ Agni, dieses ${ }^{416}$ heute wie ein Roß mit Lobliedern, «"17

11. Zum gärhapatya (wende er sich mit:) "WVenn wir Luftraum,

400 Vgl. TS III, 2, 5, 5; KS IX, 6; MS I, 10, 3; VS II, 32; VSK II, 7, 4; TB I, 3, 10, 8; MB II, 3, 8; Sānkh IV, 5, 1; GobhG ̣̣S IV, 3, 18; KhädG̣̣s III, $5,25$.

401 Vgl. TS III, 2, 5, 6; VS, VSK, TB und Sănkh we Anm. 400; ferner MB II, 3, 9; GobhGṛS IV, 3, 19; KhãdGṛ̂ III, 5, 26.

${ }^{402}$ TS, KS, VS, TB, MB, Sānkh, Gobh, Khãd wie in Amm. 400.

403 AV wie in Amm. 399; TS, MS, KS, VS, VSK, TB, Sānkh wie in Anm. 400; ferner MB II, 3, 9; Ap I, 10, 2; XIII, 12, 10; GobhG!̣S 1V, 3, 19; KhādGṛS III, 5,26 ; HirGips II, 12, 10.

401 Vgl. AV XVIII, 4, 85; MS I, 10, 3; ISS IX, 6 .

105 Nur hier.

406 In dleser Form nur hier.

407 Das hier folgende wah ist nach dem Komm, zu streichen.

408 Vgl. MS I, 10, 3; Măn I, 1, 2, 36.

409 Also RV X, 57, 3-5. Im Text ist mano statt namo zu lesen.

410 Komm.: die bef den piudas befndlichen Ahnen.

st1 Vgl. TS I, 8, 5, 2; KS IX, 6; XXXVI, 13; MS I, 10, 3.19; Ap I, 10, 7; Mān $\mathrm{I}, 1,2,14.37$.

412 Vgl. MS I, 10, 3; KS IX, 6; MB II, 3, 5.

413 Vgl. AV XVIII, 3, 14; ISS V, 3; IX, 6; MB II, 3, 5 .

11 Vgl. AV III, 20, 8; XIX, 31, 13-14; TS I, 7, 10, 1; ISS IX, 6; MS I, 10,

3. 11,4 ; MB II, 3,5 .

415 IKomm.: dem Südfeuer.

416 Nämlich Opfer. Vielleicht ist aber statt lam besser tvam zu lesen, das dem

Gebet an Agni eher den Charakter der Unmittelbarkeit verlelhen würde.

41. Vgl. RV IV, 10, 1. 
Erde oder Himmel ${ }^{418}$, wenn (wir) Mutter oder Vater geschädigt haben ${ }^{419}$, so soll Agni mich von dieser Sünde ${ }^{420}$ befreien, der Hausvater ${ }^{421}$; or soll mich schuldlos machen. ¿422 $^{428}$

12. (Mit:) »Einen Helden (-sohn) mir gebt, o Ahnen! $\aleph^{423}$ (nimmt er) von den Reismchlklössen den mittleren.

13. Er läßt (seine) Gattin ihn verzehren ${ }^{424}$, (wobei sie sagt:) "Leget in (mich), o Ahnen, eine Frucht ${ }^{425}$, einen lotusbekränzten Knaben ${ }^{42 B}$, daß er unversehrt sein mögel «227

14. Ins Wasser (wirft er) die beiden anderen (Klösse).

15. Oder in das herausgenommene (Feuer ${ }^{428}$ ).

16. Wem plötzlich ${ }^{429}$ der Appetit fehlt, der esse sie.

17. Oder wer von schwerer Krankheit befallen ist, esse sie; (dann) geht er einen anderen Weg ${ }^{430}$.

18. So (vollzieht auch) derjenige, der nicht die heiligen Feuer errichtet hat, (dieses Opfer) auf dem beständigen (Feuer ${ }^{431}$ ).

19. Nachdem er (die Opferspeise) gekocht und (das Feuer) herausgenommen hat, opfere er.

20. Paarweise (erfolgt) das Wegstellen der Opfergefäße.

418 Vil. AV VI, 120, 1; TS I, 8, 5, 3; ISS IX, 6; MS I, 10, 3; IV, 14, 17; TB III, 7, 12, 4; TA II, 6, 2 .

$\$ 19$ S. Amm. 418 außer ISS.

420 Vgl, AY VII, 64, 2; 'IS I, 8, 5, 3; III, 1, 4, 3; ISS XXXVIII, 5; MS III, 11, 10; VS XX, 14; MB II, 2, 11; TB II, 6, 6, 1; III, 7, 12, 1.3-5; TA II, 3, 1 . 6, 2; Śankh IV, 17, 12; Kāty XXV, 9, 12; Ap III, 12, 1; Kaus XLIV, 17.

s21 Vgl, AV VII, 64, 2; TS I, 8, 5, 3; KS IX, 6; MS IV, 14, 17; TB III, 7, 12, 1-6; TA II, 3, 1 .

422 Vgl. TS $\mathrm{I}, 8,5,3$; TB III, 7, 12, 1.6.

429 Vgl. Ap I, 9, 12; Mān I, 1, 2, 33.

424 Hier wird also aus der Manenspende ein im alten Indien so häuffger Fruchtbarkeitszauber.

425 Vgl. VS II, 33; MB II, 3, 16; Sảnkh IV, 5, 8; Āp I, 10, 11; Män I, 1, 2, 31 ;

Kauś LXXX1X, 6; GobhGṛS IV, 3, 27.

426 Vgl. Amm. 425 außer Gobh.

s27 Vgl. RV X, 137, 5.

428 S. II, 6, 2.

$\$ 28$ Komm.: nirnimilla ohne Ursache.

430 Komm.: er wird gesund oder er stirbt!

431 Komm, : aupäsana, das ständig brennende Hausfeuer. Der Alonenkult war also auch fǜ einen anähitägni vorgeschrieben. 
21. Gras (nimmt man) als zweites, wenn (ein Gefäß) überzählig ist. ${ }^{432}$

\section{Ende der 7. kaṇ̣ikia}

II, 8

1. Wer ein Neu- und Vollmondopfer ${ }^{433}$ in Angriff nehmen will, (führe zunächst) die anvärambhanŭyā (-iș/i ${ }^{434}$ durch).

2. (Die Gotheiten sind) Agni-Viṣụu, Sarasvati, Sarasvant (und) Agni Bhagin.

3. „O Agni-Visnu, es sollen euch diese ${ }^{435}$ Sprüche eimmütigg ${ }^{46}$ begeistern ${ }^{437}$; mit Herrlichkeit und Gütern kommt herbei !438 O Agni-Vișnu, groß ist eure angenehme Stätte ${ }^{439}$; ihr genießt von der Schmelzbutter, am Verborgenen euch erfreuend ${ }^{440}$; in jedes Haus kommt eure schöne Lobpreisung ${ }^{441}$. Hinan zu der Schmelzbutter möge sich eure Zunge bewegen. ${ }^{442}$ Die strahlende Sarasvatī unser ....43; die blitzgeborene Tochter von ausgezeichneter Lebenskraft ${ }^{44}$. - Sarasvants schwellenden ...445. Den himmlischen, schöngeflügelten Vogel, den großen . . . ${ }^{440}$ - Herbei als den Anreger Savitars $\ldots{ }^{447}$ Als solcher bring uns Gaben herbei ${ }^{448},{ }^{449}$

$\$ 32$ Die sütras 19-21 enthalten die Besonderheiten, die ein anāhitägni bein pinda-Opfer za beachten hat. - Hiermit schließt die Bchandlung des pindapitryajina.

4a3 Ausfithrlich dargestellt in adhyāyă I. 23,4 .

${ }^{495}$ Diese wird im Folgenden als selbständiger Ritus behandelt. Vgl. Ãp V,

435 Die Worttremung sajoșase $m a ̈$ ist aufzuheben.

${ }_{436}$ Vgl. TS IV, 7, 1, 1; V, 7, 3, 2; MS IV, 10, 1. 11, 2; TB III, 11, 3, 1; Ap XVII, 17, 8; Mān V, 1, 1, 33.

437 Vgl. TS und 'TB lt. Amm. 436; ferner MS IV, 10, 1.

$438 \mathrm{Vgl}$. Anm, 437.

439 Vgl. AV VII, 29, 2; TS I, 8, 22, 1; ISS IV, 16; MS IV, 10, 1. 11,2; ÁsV V, 19, 3; Śănkh II, 4, 3; Mān V, 1, 6, 26.

440 S. Anm. 439 außer MS IV, 11, 2; Aśv V und Măn.

44 S. Anm. 439 außer MS IV, 10, 1; Asv V und Mān.

413 S. Anm. 439 auBer MS IV, 11, 2; Āśv V und Mãn; zzgl. KB VII, 2.

4*3 RV I, 3, 10.

14 RiV VI, 49, 7.

45. RV VII, 96, 6.

46 RV I, 164, 52.

447 RV VIII, $102,6$. 
4. Wenn man nach der Errichtung (der heiligen Feuer) krank wird oder wenn sich die Besitztümer vermindern, (erfolgt) bei der erneuten Errichtung ${ }^{450}$ eine $i s ̦ t i$.

5. Bei dieser soll er ${ }^{461}$ die Voropfer ${ }^{452}$ und Nachopfer ${ }^{463}$ mit den Kasus ${ }^{454}$ darbringen:

6. „Die Brennhölzer, die Brennhölzer - o Agni455 - o Agni, sollen von dem Opferschmalz genieß3en ${ }^{456}$. Tanünapăt ${ }^{457}$ den Agni ${ }^{458}$ - o Agni, soll von dem Opferschmalz genießen ${ }^{459}$. Die Opferspenden - durch den $\mathrm{Agni}^{460}$ - o Agni, sollen von dem Opferschmalz geniessen ${ }^{461}$. Die Opferstreu - der Agni ${ }^{462}$ - o Agni, soll von dem Opferschmalz genießen ${ }^{463} . "$

7. "Mit Brennholz dem Agni huldigt ${ }^{404}$; komm her, sogleich will ich für dich rezitieren ${ }^{465} \ll$, so (lauten) die beiden auf Agni bezüglichen äjyabhägata6 (-anuwãkyāis ${ }^{467}$ ).

448 RV VII, 15, 11.

449 Es werden jeweils die pratikas einer yäjya und einer anwakya gegeben (Komm.), und zwar richten sich die Sprüche in Amm. 435 bis 442 an Agni-Vişņu, in Amm. 443 bis 444 an Sarasvati, in Anm, 445 bis 446 an Sarasvant, und in Anm. 447 bis 448 an Agni Bhagin. - Hiernit schliebt die Darstellung der anvärambhanilya-işil.

4so Vor diesem sütra hätte clne kạdikä-'Teilung erfolgen kömnen, denn es kommt nunmehr eine neuc Zeremonie zur Erörterung, deren Platz zweckmäßigerweise nach II, 1, 36 gewesen wäre; vgl. Āp V, 26 f.

451 Der holar.

45. S. ZMR Anm. 448.

453 S. ZMR Anm. 164.

454 Gemeint ist (s. auch das folgende sūtra) die Einschiebung des Götternamens Agni in verschiedenen Kasus; vgl. $\lambda_{p} V, 28,8$.

455 1. vibhakti (Vok.).

456 Vgl. Săn̉kh I, 7, 1; Mān V, 1, 2, 6.

457 Eine Form des Agnt.

458 2, vibhakl (Akk.).

459 In dieser Form nur hier.

$\$ 60$ 3, vibhakti (Instr.).

461 S. Anm. 459.

463 4. vibhaklt (Nom.).

463 S. Anm, 459.

464 RV VIII, 44, 1. Im T'ext ist duhaspate hyạsu in duvasyata ehy $\bar{u}$ șt zu ändern.

465 RV VI, 16, 16.

486 S. ZMR, Amm. 86 zu I, 3, 8.

${ }_{467}$ Komm, - S. ZMR Amm. 195 zil I, 5, 29. 
8. Als "weise« und "somatropfend" werden die beiden bezeichnet.

9. Entsprechend ist die Gültigkeit (der Regel in den nigamas ${ }^{488}$ ).

10. Und in der $y \bar{a} j y \bar{a}^{469}$ (erfolgt ebenfalls die Nennung der Eigenschaften der Gottheiten ${ }^{470}$ ).

11. Obligatorisch (ist) im ersten (äjyabhäga Agni die alleinige

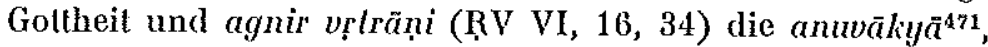
so sagen) die Anubrähmanin (-Lehrer).

12. »O Agni, du läuterst die Leben ${ }^{472}$ ist beim zweiten (äjyabhăga die anuvāky $\left.\bar{a}^{473}\right)$.

13. Obligatorisch ist aber im zweiten (yajjyamantra ${ }^{474}$ ) das Wort havis ${ }^{475}$.

14. (Denn) Agni-artig ist das havis. "Denn, o Agni, des edlen Willens ... « ${ }^{478} ;$ »Dich mit diesen Liedern heute preisend « ${ }^{477}$; - »Durch diese unsere Preisungen ... «478; »O Agni, dies (Opfer) heute wie ein Roß mit Lobsprüchen . . . « ${ }^{\text {d79 }}$, - dies sind die beiden sainyäjyäs ${ }^{480}$. "Gott Barhis - des Agni ${ }^{481}$ genieße für reichlichen Erhalt der Reichtumsgabe ${ }^{\$ 82}$; Gott Naräśaniosa - bei Agni $^{483}$ - genieße für reichlichen Erhalt der Reichtumsgabe ${ }^{484} \cdot «^{485}$

Ende der 8. kaṇḍika

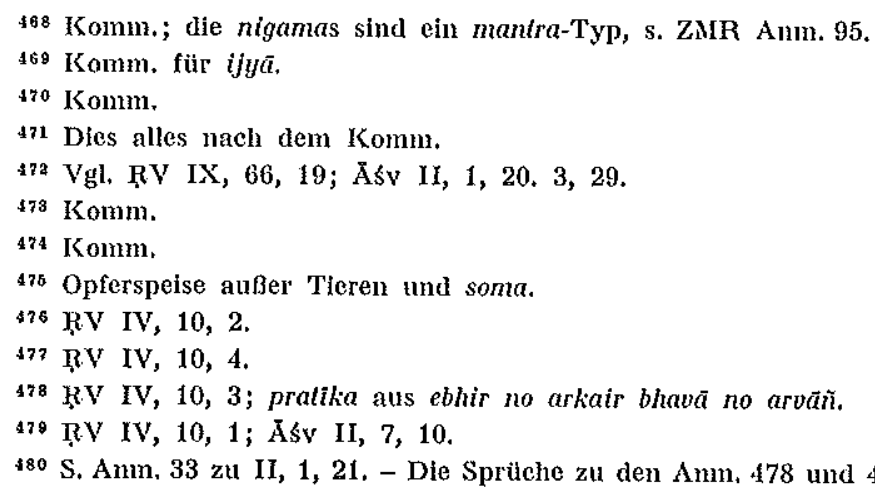
Agni Svișțakṛt gewidmet; die beiden vorhergehenden sind die yäjyānuvākyā an Agni.

481 vibhakti (Gen.), s. Anm. 454 zu II, 8, 5.

482 Vgl. Säinklı II, 5, 19 und ZMR Anm. 281.

483 vibhakli (Lok.).

484 Vgl. Sảnikh II, 5, 19 und ZMR Anm. 282.

483 Die letzten beiden Spriche sind durch die vibhakits als anuyajas gekemzeichnet. - Hiermit schliebt die Behandlung des punarädheya. 
II, 9

1. Das Erstopfer (von Feldfüchten bestehe jeweils) aus Reis, Hirse $^{488}$ und Gerste ${ }^{487}$.

2. Von der (neuen) Ernte esse man nicht, ohne das agnihotra geopfert zu haben ${ }^{488}$.

3. Wenn (dic Erde) vom Regen gesättigt ist, dann opfere man mit der Erstlingsfrueht ${ }^{489}$.

4. Selbst die Götter nämlich sprachen: "Darum soll man, gesättigt vom Regen, mit dem ägrayana opfern. ${ }^{490}$ - Oder man füttere $^{491}$ die agnihotr $a$-Kuh mit diesen (Feldfrüchten) und opfere mit ihrer Milch.

5. Ferner (erfolgt das Opfer) mit Gerste oder (es geht) ohne (diese) Zeremonie ${ }^{492}$.

6. Das Opfer (mit Gerste ist) aber (die Pflicht) eines Königs.

7. Und (es ist eine Pflichl) für alle, (meinen) manche (Lehrer).

8. Beim Hirseopfer (wird) ein Soma gehörender Reisbrei (dargebracht).

9. „O Soma deine erquickenden . . . ${ }^{493}$; deine Wohnstätten im Himmel und auf der Erde .... $\wedge^{494}$ - (rezitiert der holar). Zur aväntared $a^{895}$ spricht er die übliche geflüsterte Formel ${ }^{496}$, nimmt (die avāntaredă) in die linke Hand und berühre (sie) mit der anderen (wobei er spricht:) "Für Prajāpati zur Annahme ergreife ich dich, mir zum Glïck, mir zum Ruhm, mir zur Nahrung. « ${ }^{497}$

486 Panicum frumentacetm.

487 Mit diesem sütra beginnt die Darstellung eines neuen Opfertyps, der ägrayaṇești; Vğl. Äp VI, 20-31; Kāty IV, 6; BhārŚrs VI, 15-18; SBE XII, S. 369; B. Lindner im FestgruB für Böhtlingk, S. 79 ff.

488 Komm.: mit Opferspeise aus der neuen Ernte.

489 Komm.: mit Reis zur Herbstzeit.

490 Elne brálmana-älmbliche Passage.

491 Die im Text enthatlene Worttrennung zwischen val und nänadayitva ist. aufzuheben; bei Auflösung des Sandhi ist vă enân adayitvā zu lesen.

‘sz D. h, man hat hier freie Wahl. Im Text ist väkriyä (= vä akriyá) stall vã kriyă zu lesen.

s93 RV I, 91, 9; vgl. Áś IV, 4, 4; X, 6, 6.

40 RV I, 91, 4; vgl. Ảsv III, 7, 7; IV, 3, 2 .

495 S. ZMR Anm. 243.

406 Konm.: iḍe bhăgam; vgl, TB III, 7, 5, 7; Ӑp III, 2, 11; Åsv I, 7, 8.

497 Der Spruch erseheint sonst nur noch SāṅhhG̣̣S III, 8, 2. 
10. "Vom Guten habt ihr uns zum Besseren geführt, o Götter «498. "Durch dich als Hilfe möchten wir dich erlangen ${ }^{499}$. "In unsere Nahrung tritt erquickend hier ein $\left\lfloor «^{500} »\right.$ Sei ein Segen für uns, den Zweifüßler, Segen für den Vierfüßler!«501 (Danach) verzehre er (die abāntarcọă), späle (den Mund) aus, berühre (seinen) Nabel (und spreche:) »Ein Ungestüm bist du, o Atem, diese VVahrheit spreche ich aus, ${ }^{602}$ Ein Ungestium bist du, in alle (Wesen) eingetreten ${ }^{503}$. Der du Alter und Krankheit von meinem Körper verbannt hast ${ }^{504}$, komm heim zu mir, vergiß uns nicht, o Indral« ${ }^{505}$

11. Mit diesem (mantra sollen) die Speisen Verzehrenden ${ }^{606}$ uberall bei der neu (geernteten) Speise (vorgehen).

12. Die bei der (Opferung von) Reis und Gerste eingeschobenen Verse ${ }^{507}$ sind (metrisch) vir $j^{508}$.

13. (Die Gotheiten dieses Opfers sind) Agni-Indra oder IndraAgni, die Allgötter; Soma, wenn es sich um das Hirseopler handelt; (und) Dyävāpṛthivī.

14. (Der holar rezitiert:) »Herbei die das Feuer entzinden« ${ }^{509}$; »Die gute Werke Verrichtenden, schön Strahlenden, (andere) Götter Verehrenden . . . « ${ }^{510}$. - $\gg$ O Allgötter, kommt herbei« ${ }^{511}$. "Und die, welehe auf der Erde als mächtige, vielgestaltige $\ldots \ll^{512}$ - »Dic beiden Mächtigen, Himmel und Erde, ums

408 Vgl. TS V, 7, 2, 4; KS XIII, 15; MB II, 1, 13; TB II, 4, 8, 7; А̄p VI, 30, 8; Män I, 6, 4, 25; Śänihgịs III, 8, 3; PūrGṛS III, 1, 4; Kaus LXXIV, 19.

199 S. Anm, 498 auber $\bar{\lambda}$.

500 S. Anm, 498 außer KS, $\lambda_{p}$, Kauś.

501 RV VII, 54, $1 ; x, 85,43-44$.

so2 Vgl, MB II, 1, 14; SảnkhGṛS III, 8, 4; Iauś IXXIV, 20.

503 Vgl. Katus LXXIV, 20.

so: S. Anm. 502.

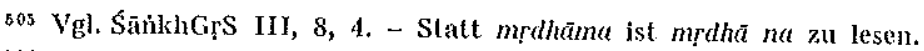

sos Gilt natiurlieh nur für elnen hotar.

${ }^{807}$ dhāyyā; vgl. Āśv II, 1, 27.33; Āp VI, 31, 18; XIX, 18, 3.

${ }^{508}$ Ein Metrum mit meist 3 zelm-, seltener elfsilbigen pădas.

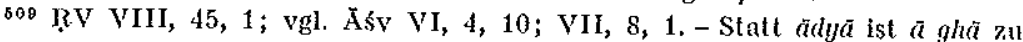
lesen.

510 RV IV, 2, 17.

s11 RV I, 3, 7; II, 41, 13; VI, 52, 7.

s12 RV VI, 52, 15; vgl. Åsv III, 7, 10. - Statt jmämahi no ist jmā mahino zu lesen.

$0 *$ 
... $\ll^{513} »$ Die in der Vorzeit geborenen Eltern mit ganz neuen $\ldots \ll^{514}$.

Ende der 9. kaṇdikă

II, 10

1. Nun (werden die) auf (die Erfüllung) eines Wunsches ${ }^{515}$ bezüglichen ( $\mathrm{O}_{\mathrm{p} f e r}$ besprochen).

2. Die in dem WVunschopfer für (langes) Leben (gebrauchten beiden äjyabhäga-anuvākyäs ${ }^{516}$ heißen) »Leben enthaltend«.

3. (Sie lauten:) $\gg \mathrm{Zu}$ uns, o Agni, mit Wohlwollen ... $«^{517}$ (und) "Du, o Soma, dem Bejahrten Glück ...." ${ }^{518}$ (Die angerufenen Gotheiten sind) Agni als äyușmant, Indra als trătar.

4. „Leben möge dir von allen Seiten geben ${ }^{519}$ dieser Agni, der herrliche ${ }^{520}$; dein Atem soll wiederkehren ${ }^{521}$; hinweg treibe ich dein kachektisches Leiden ${ }^{522}$. Leben spendend, o Agni, an der Opferspende (dich) erfreuend ${ }^{523}$, sei schmelzbuttergesichtig mit einem Schmelzbutter-Schoß ${ }^{524}$. Nachdem du Schmelzbutter getrunken hast, den angenehmen, von der Kuh stammenden Honig ${ }^{525}$, beschütze wie ein Vater den

${ }^{513}$ RV I, 22, 13; vgl, Ass I1, 16, 2; III, 10, 24. 11, 20; VI, 5, 18; VIII, 10, 2.

s14 RV VII, 53, 2, - Es handelt sich hier durchweg um die vom hotar zu rezilierenden anuuākyās und yäjyās, vou denen das erste Paar an Agni-Indra, das zwelte an die Visve Devăh (Allgötter), das dritte an Himmel und Erde (Dyãvãpṛthivi) gerichtet ist. - Hiermit schliebt die Besprechung der ägrayaneşi.

615 Es handelt sich hierbei im Gegensatz zu den bisher besprochenen Riten um aperiodische Wunschopfer, die ohne scharfe Grenze in eigentliche Zauberhandlungen itbergehen; vgl. Āp XIX, 18-27.

516 So erklärt es die Pray.

s17 RV I, 79, 9.

518 RV $\mathrm{I}, 91,7$.

s19 Vgl, TS I, 3, 14, 4; TA II, 5, 1. 7, 1; Д̆p XIX, 24, 9; HirGṛ I, 5, 15; II, 4, 19; BaudhDhS III, 7, 10.16 .

s20 Vgl, AV VII, 53, 6; TS I, 3, 14, 4; TA II, 5, 1; SảnihhGṛS I, 25, 7.

621 Vgl, TS I, 3, 14, 4; TA IX, 5, 1 .

${ }^{322}$ RV X, 137, 4. Dle Übersetzung von yakșma ist nur in dieser allgemeinen Form möglich.

s23 Vgl. TS I, 3, 14, 4; III, 3, 8, 1; TB I, 2, 1, 11; TA II, 5, 1; Аp XIII, 19, 10 ; S̊ảnkhGṛS I, 25, 7.

624 Vgl. 'TS I, 3, 14, 4; III, 3, 8, 1; VS XXXV, 17; SB XIII, 8, 4, 9; TB I, 2,

1, 11; TA und SānkhGṛs wie in Anm, 523.

625 Vgl. AV II, 13, 1; sonst wie Anm. 524. 
Sohn diesen ${ }^{526}$ (Opferveranstalter). - Indra den Retter, Indra den Helfer . . .527. Nicht, o Mächtiger, in dieser Bedrängnis . . . ${ }^{528}$ - Beschüitze uns, o Agni, mit unanfhörlichen Hilfeleistungen ${ }^{629}$; o Agni, beschütze du uns auf's Neue ${ }^{630} \ll$, so (lauten) die beiden sainyājyäs $\mathrm{s}^{531}$.

5. Bei einem Opfer um die Erlangung von Segen (heißen die beiden äjyabhăga-anuvākyă(s ${ }^{532}$ ) »schützend«.

6. (Sic lauten:) $» A g n i$, schütze uns vor Bedrängnis! $\aleph^{533}$ (und) "Du uns, o Soma, von allen Seiten ....(«34

7. 'Segen uns vom Himmel, o Agni, von der Erde $\ldots{ }^{535}$; fern von uns die Not, fern die Bedrängnis ${ }^{536}$. Die sainyājyās sind die oben erwähnten.

8. Bei einem Sohneswunschopfer ist Agni Putrin (die Gottheit).

9. «Dem Frommen, o Jãtavedas $\left\langle^{537}\right.$; $\gg A n$ dich mit lobsingendem Herzen denkend « ${ }^{538}$ (sind hierbei anwāalyā und yajjyä). Die beiden mit "Agni einen hochberühmten ... « ${ }^{639}$ (beginnenden Verse) sind die sainyājyās.

10. Auf Agni ${ }^{540}$ bezogen (sind) die beiden folgenden ${ }^{541}$ (Opfer).

526 S. Amm. 525.

${ }^{527}$ R.V VI, 47, 11; vgl. Åśv VI, 9, 5 ,

598 RV VII, $19,7$.

s20 RV I, 189, 4; vgl. Áśv III, 7, 5.

${ }^{530}$ RV I, 189, 2.

s91 Die Sprüche von Anm. 519 bis 522 sind die anuväkyä, von 523 bis 526 dle yajya an Agni Ayuşmint; Anm. 527 ist antwākyā, Anm. 528 yājyä an Indra Trătar. 529 und 530 sind die analogen Sprüche an Agni Sviștakṛt; s. Amm. 33 zu II, 1, 21 .

332 S. Anm. 516 zu II, 10, 2.

533 RV VII, 15, 13.

sง: RV I, 91, 8; $\mathrm{X}, 25,7$.

${ }^{635}$ RV X, 7, 1; antwäkyä beim eigentlichen Opfer.

s36 RV IV, 11, 6; yäjyä ebenda.

sง? RV V, 4, 11.

338 RV V , 4, 10.

s39 RV V, 25, 5-6. Statt agnistu viśravastamam ist agnis tuviśravastamam zu lesely.

540 Und zwar nach Komm. und Pray. in den Aspekten des Agni Müdhanvant und Agnl Kāma.

541 Wetchen Zwecken diese dienen sollen, sagt $A s ́$ vicht, kamn aber aus Paralle\}stellen, besonders Sannkh III, 4, 10; BaudnŚrS XIII, 5; Hir XXII, 2, 14, erschlossen werden: es landelt sich um die Realisierung eines bisher nicht erfülten Wunsches. 
11. Die beiden obligatorischen (Verse ${ }^{542}$ ) enthalten ${ }^{543}$ clas Wort "Haupt!«

12. "Zu dir diejenigen, o bester Angiras . . . $\ll^{544}$; $»$ Erlangen wollen wir diesen Wunsch, o Agni, mit deiner Hilfe ${ }^{545}$ - clies (sind anubākyā und yăjyā) zur Wunscherfüllung ${ }^{546}$.

13. Auf Indra ${ }^{547}$ bezogen (sind) die beiden folgenden ${ }^{548}$ (Opfer).

14. "Zerschlage, o Indra, unsere Feinde ${ }^{529}$; "Wie cin wildes Tier, gewaltig schreitend, im Gebirge hatsend . . . $\ll^{550}$. »Den ${ }^{551}$ Verliebten ${ }^{552}$, o Indra, coniunctum cum ciaculatione seminis $^{553}$, fluentem ${ }^{554}$, ad eam, cuius vulva humorem stillat ${ }^{555}$, zum goldglänzenden Mädchen führe uns $!^{556}$ Ut penem immittens inter femora ${ }^{557}$, zerschlage, o Indra, unsere Feinde $\left.\right|^{558}$ canikhudad $^{559}$ futuens $^{560}$. Zu guter Preisung geleite uns! ${ }^{561}$

\$12 Nach Komm. und Pray, sind dies s\gni, das Haupt, des Himmels Gipfol (RV VIII, 44, 16) und „Der Erde, des Opfers und des Luftraums Fihrer' (RVX, 8, 6). ${ }_{513}$ D. h. bei demjenigen der beitlen Opfer, das Agni Mūrdhanvant zur Got heil hal.

s4s RV VIII, 43, 18; vgl. Áśv IIII, 10, 4.

${ }_{513}$ RV VI, 5, 7. Es ist atsyama tam statt aślamantam zu lesen.

546 Hier ist also Agni Kãma die Gottheit.

517 vaimplha ist ein Aspekt des Gottes Indra.

s4s Sie dienen nach dem Komm. dem Gedeihen (puşi), doch dem Zusammenhang nach eher der Abwehr von Feinden.

810 RV $\mathrm{X}, 152,4$.

550 RV 1, 154, 2; X, 180, 2. Die Quelle für $\ddot{X} 5 \mathrm{~V}$ ist hier $X, 180,2$, da sich die erstgenannte Stelle an Vişn, wendet.

${ }^{551}$ In dieser Form nur hier, - Es beginnt her eine z. T. mil TB II, 4, 6, 5. 5, 3, 1 ubereinstimmende, nur versuchsweise ibersetzte Stelle, die stark verderbt, möglicherweise aber auch absichtlich dunkel gehalten, jedenfalls inlerpoliert ist. Sie stellt einen Liebeszauber dar, der von dem eigentlichen Charakter des Opfers völlig abweicht.

${ }^{652}$ Mit. TB liest man besser sakulim statt sadyulim.

${ }_{563}$ Nach dem ISomm. zu MS II, 7, 12.

sst TB hat sacyulim für pracyutim.

555 Nach dem kleinen PV.

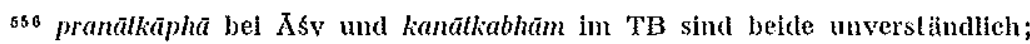
die obige Ubersetzung stiltzt sieh auf den Komm, zum TB.

557 TB II, 4, 6, 5 .

558 S. Anm. 549.

559 Ebensowenig deutbar wie das kanikhunad im TB; die Zurückführung auf die Wz. khan im Komm, zu 'TB ist ganz wllkürlich. Eher denklsar wäre ein Intensivpartizip der Wz, khud identldem futuense. 
15. Für Indra, den Geber oder den Wiedergeber (opfere, wer $\ldots{ }^{562}$ )

16. "Die unsere Besitztümer sind ${ }^{563}$, zornig bringst du uns um diese voller Grimm ${ }^{564}$. O Indra, laß uns ihrer habhaft werden $^{565}$ durch diese Opfergabe wieder ${ }^{506}$. Wieder soll uns Indra, der Gabenreiche, (sie) geben ${ }^{567}$, dic Besilztümer, der Starke $^{508}$, der Schätzereiche ${ }^{569}$, der Freigebige ${ }^{5 \% 0}$. Uns zugewandt möge der Erwünschte (seinen) Sinn gestalten ${ }^{5 n 1}$. Gern möge Indra durch unsere Opfergabe (uns) vergessen ${ }^{572}$ «

17. Oder (man opfere ${ }^{573}$ ) den Hütern der Himmelsrichtungen ${ }^{574}$.

18. "Den Hütern der Himmelsrichtungen ${ }^{575}$, den vier Unsterblichen $^{570}$, den Aufsehern der Schöpfung ${ }^{577}$, wollen wir dies als Opfergabe verehren ${ }^{578}$. - Alle Himmelsrichtungen mit Honig ich vereinige ${ }^{579}$. Gesund ${ }^{580}$ sollen Wasser und Ge-

soo Nach dem TB, das sapayan hat.

so1 TB II, 4, 6, 5 .

${ }_{562}$ Welchem Zweek das Opfer dient, wird nicht gesagl; oflensichtlich handelt es sich um den Erhalt von Gaben; nach BaudhSrS um den Empfang von Gaben seitens der Untertanen (eines Königs).

${ }^{663}$ In dieser Form nur hier.

s6t S. Anm, 563 .

563 S. Anm. 563.

s8B Vgl. TB II, 5, 3, 1 .

$367 \mathrm{Vgl}$ AB VII, 21, 2; TB II, 5, 3, 1 .

568 sakra $=$ Indra.

${ }^{569}$ dhanyah nach TB ist besser als dhanih bei $\bar{A} s \mathrm{v}$.

570 TB II, $5,3,1$.

s71 S. Amm. 503.

${ }^{572}$ Der Sim ist vielleicht: er möge sich iber dem Verzehr der Opfergaben nicht nehr mit dem ülsrigen Besitzlum der Bitlenden befassen. Da aber die TBParallelstelle hier stärker abweicht, scheint mydhäli doch verderbt zu sein.

573 S. $\Lambda$ nm. 562 zu II, $10,15$.

sis Der Komm. faßt ăsá bzw. die ăsiapaaläh als Gotheit dieses Opfers, doch ist das weder grammatisch noch mythologisch gerechtfertigt.

$678 \mathrm{Vgl}$. AV I, 31, 1; TB II, 5, 3, 3; III, 7, 5, 8; Ӓp IV, 11, 1; VII, 16, 7 .

536 S. Anm, 575 außer Ap VII.

577 S. Anm. 576.

578 Vgl. AV I, 31, 1; VI, 41, 1-2; 'TS IV, 2, 9, 2; KS XVI, 16; MS II, 7, 15; VS XIII, 21; TB II, 5, 3, 3; III, 7, 5, 9; SB VII, 4, 2, 15; TA X, 1, 8 ; А IV, 11, 1. Bis hier die anuväkyä.

579 Vgl. TB II, 5, 3, 3; Äp VII, 16, 7.

s80 Es ist anamivã statt anami vã zu lesen. 
wächse alle $\operatorname{sein}^{581}$. Dieser Opferveranstalter soll (seine) Feinde zerstreuen ${ }^{582}$; nicht geraubt soll all sein Vieh $\operatorname{sein}^{587}$."

19. Das Weltopfer (folgt num ${ }^{684}$ ).

20. Die Erde, der Luftraum (und) der Himmel sind die Gottheiten.

21. „Die Erde, die Mutler Erde ${ }^{585}$, den Luftraum rede ich an ${ }^{586}$. Zu Hilfe den hohen Himmel ${ }^{587}$ Alles erhält die Erde ${ }^{588}$. Den Luftraum hat sie sich ausgebreite $t^{589}$. Es läßt der hohe Himmel Milch strömen ${ }^{690}$. Schutzwehr (sei) mir die Erde, das Land ${ }^{591}$, dem Luftraum zum Heil ${ }^{592}$. Der Himmel sei mein Schulz, groß (mein) Ansehen ${ }^{593}$ « Diese drei (Verse gehören jewcils) zu den drei (Gottheiten) $)^{694}$.

22. Die ersten beiden (Verse gehören) dem ersten ${ }^{595}$, die letzlen beiden dem mittleren $^{596}$.

Ende der 10. kaụ̣̣ikā

II, 11

1. Das Freundgewimnungs(-opfer) ist eine große mit dem viräjMetrum verbundene (Zeremonie).

681 S. Anm. 579 sowle HirGṛS II, 5, 3.

582 S. Anm. 579 .

ss3 S. Anm, 579. Bis hier die yäjyā.

58! S. Anm. 562 zu II, 10, 15.

585 Vgl. TB II, 4, 6, 8.

586 Vgl. TB II, 4, 6, 8.

887 Vgl. TB II, 4, 6, 8.

$588 \mathrm{Vgl}$. TB II, 4, 6, 8.

s80 Vgl. TB II, 4, 6, 9.

$500 \mathrm{Vgl}$ TB II, 4, 6, 9.

$50 x$ Nur an dieser Stelle.

522 Nur an dleser Stelle.

593 Nur an dieser Stelle.

594 Die Pray. zeigt, wie dies za verstehen ist: Die Sprüche in Anm, 585 bis 587 gelten als erster, die in Anm, 588 bis 590 als zweiter, die in Amm. 591 bis 593 als dritter Vers. Die ersten beiden sind anuwäkyā und yājyā für die Erde, der zweite und der dritte für den Luftraum, der dritte und der erste für den Himmel. Dies zeigt angedeutet auch das folgende sütra.

593 Also der Erde.

s96 Also dem Luftraum. - Es bleibt demnach fir den Himmel nur eine Konbination der Verse 1 und 3. Ein großartiges Beispiel für die Prägnanz der Sraulasūtras! 
2. Agnni, Soma, Varuṇa, Mitra, Indra, Bp̣haspati, Savitar, Pūșan, Sarasvati (und) Tvaștar sind Empfänger einer einheitlichen Gabe.

3. (Die anuvāliyă lautet:) Indra ${ }^{698}$, Bịhaspati, Savitar, der tausendfach gewähr ${ }^{599}-$ Pŭșan soll uns mit Rindern, mit Förderung600 Sarasvatín ${ }^{601}$, Tvaștar mit Gestalt ausstatten zum Opfer ${ }^{602}$.

4. Er opfere ${ }^{603}$, nachdem er (die Gotheiten) in ungekehrter Reihenfolge aufgeführt hat: $" W i r$, die wir opfern ${ }^{604}$ für Tvaștar, Sarasvatī, Püșan, Savitar, Ḅ̣haspati, Indra, Mitra, Varuṇa, Soma, Agni. - Tvaștar ${ }^{605}$ soll uns Gestaltungen, die freigebige Sarasvatī ${ }^{600}$, Pūşan und Savitar Wohlstand ${ }^{607}$, Bṛhaspati und Indra mögen tausend(-fachen Wohlstand) geben ${ }^{608}$, Mitra der Geber, Varuma, Soma, Agni ${ }^{609}$."

5. Die acht (mit "Auf Agni bezogen (sind) die beiden folgenden (Opfer) $«^{810}$ beginnenden Zeremonien ${ }^{611}$ ) haben (lurchlaufend das virä $j$-Metrum.

6. Von diesen haben die ersten sechs eine einheitliche Opfersubstanz.

7. Mit dem "Schwiegertochter-Schwiegervater-Opfer" opfere (jemand, (ter einen Feind ${ }^{612}$ ) behext ${ }^{613}$.

\footnotetext{
597 Komm,

${ }^{598}$ Vgl. 'IB II, 5, 3, 3; SB XI, 4, 3, 6; Śankh III, 7, 4; Kāty V, 12, 20.

590 S. $\Lambda \mathrm{mm}, 598$.

600 Man beachte den symmetrischen Satzbau; ähnlich: "Sic sinkt mil euch. Mit euch wird sie sich hebens (Schiller).

Bo1 S. Anm. 598.

602 In dieser Form nur hier.

${ }^{803}$ Es muß yajel heißen.

$60 \%$ S. ZMR Aim. 174.

b05 Hier beginnt nach dem Komm. die yäjyä.

606 Vgl. TB II, 5, 3, 3; SB XI, 4, 3, 7; Sãủkh III, 7, 4; Kāty V, 12, 21.

${ }_{807}$ In dieser Form nur hier.

608 A. Anm. 606.

809 Vgl. TB II, 5, 3, 3 .

${ }^{610}$ S. II, 10, 10.

b11 Komm.

612 Komm.

413 Komm. hat gar märayami (ich töte).
} 
8. "Indra, der Held, durchquerte die Luftäume ${ }^{614}$. Die Schwiegertochter ist die Feindin ${ }^{615}$, ich bin der Schwiegervater ${ }^{616}$. Ich besiege die Feinde, mich schr begeisternd ${ }^{617}$. Ich gewinne den Preis im WVettkampf ${ }^{\beta 18}$. Indra, der Held, (war) als erster der Allwirkende ${ }^{619}$. Lr, der die Maruts als Gefolge hat, soll ${ }^{620}$ Scharenherr sein mit Edelgeborenen ${ }^{621}$. Meine Schwiegertochter soll als Feindin auf Befehl ${ }^{622}$ des Schwiegervater's $^{623}$ (dessen) Rede mit dem Geist ${ }^{624}$ abwarten ${ }^{625}$."

9. »Als angenehmer Hausfreund ... $\ll^{626}$ (und) $» O$ Agni, erstarke zu großer Herrlichkeit $\ll^{627}$ (sind) die beiden sainyaujyàs.

10. Zur Erzielung eines Einverständnisses mit Andersgesinnten (vollziehe man) das Eimmütigkeitsopfer.

11. Agni der von der Vasus Begleitete, Soma der von den Rudras Begleitete, Indra der von den Maruts Begleitete, Varuna der von den Ädityas Begleitete erhalten eine gemeinschaftliche Opfergabe.

12. "Möge Agni als erster mit den Vasus uns schützen ${ }^{628}$; Soma soll mit den Rudras (uns) recht beschülzen ${ }^{629}$; Indra soll

G14 Vgl, 'TB II, 4, 6, 12.

615 Hier beginnt eine Reihe von Zaubersprichen, deren Sinn im System der altindischen patriarchalischen Großfamille begründet liegt, das $u$. a. eine völlige Unterwerfung der Schwiegerlochter unter den Schwiegervater vorsieht. Der als Schwiegertochter deklarierte Feind ist damit der Unterwerfung ansgeliefert; vgl. AB III, 22, 7.

ete S. Anm, 614.

617 In dieser Form nur hter.

as In dieser Form nur hier.

619 Nur an dieser Stelle.

620 Es ist astu statt asru zu lesen.

621 In dieser Form nur hier.

${ }^{622}$ Es ist praśiștau statt praviștau zu lesen.

\&23 S. Anm, 621.

62: Nach der TB-Parallelstelle, die manas $\bar{a}$ hat.

${ }^{623}$ S. Anm. 614. - Auf anuvakyā und yäjyä folgen nun noch die suiștakrt. Sprüche.

626 pralika aus jușlo damūna atilhir durọna RV V, 4, 5; Āś II, 12, 5. 18, 17.

627 RV V, 28, 3; Āsv II, 18, 17.

628 Vgl. TS II, 1, 11, 2; KS X, 12; MS IV, 12, 2; Sānkh III, 6, 2.

B20 S. Anm. 628 . 
mit den Maruts ordnungsgemäß handeln ${ }^{630}$; mit den Ãdityas möge uns Varụ̣a Schutz gewähren ${ }^{631}$. Möge Agni zusammen mit den Vasus uns schützen ${ }^{632}$; Soma mit seinen RudraScharen ${ }^{833}$, Indra, der zum Opfer bereite, zusammen mit den Maruts ${ }^{034}$, zusammen mit den Ädityas der allwissende Vartua ${ }^{635}$ «

13. Das Indra-Marut-Opfer (darbringen sollen dicjenigen,) die Spaltung ${ }^{636}$ wünschen ${ }^{637}$.

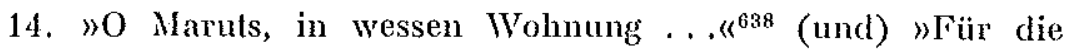
Marut-Schar, die selbstleuchtende . . . ${ }^{639}$

15. Nachdem er die Indra-antwākyä gesprochen hat, spreche er die Marut- $y a \overline{a j} y a \tilde{a}$; nachdem er die Marut-anubäkyä gesprochen hat, spreche er die Indra-yajy $\bar{a}^{040}$. In den nigamas ${ }^{041}$ (cr'wähne er) Indra zuerst oder die Maruts.

16. Oder (er nennt zuerst) Indra (vor dem Hauptopfer); nach dem Hauptopfer die Mauts ${ }^{6: 2}$.

17. Nach der Grundregel (mögen diesen beiden Gollheiten ${ }^{643}$ diejenigen opfern,) die gemeinsamen Wohlstand ${ }^{644}$ wollen, und ein Einigkeits(-opfer anschließen).

18. Das Indra-Bṛhaspati- $\left(\mathrm{O}_{\mathrm{P}} \mathrm{fer}\right.$ möge darbringen), wer (von Feinden $\left.{ }^{845}\right)$ angegriffen wird.

630 S. Amm. 628.

631 S. Anm. 628.

${ }^{632} \mathrm{~S}$, Anm. 628 außer TS.

633 S. Anm. 632 .

${ }^{634}$ S. Anm, 632.

o3s S. Anm, 632 .

${ }^{6 a}$ Komm.; zwischen dem König tnd den Unterlanen (viśs).

637 Man ersieht hieraus, daß das altindische Opferritual zur Staatsangelegenheit werden komnte

68. R.V I, 86, 1; vgl. Aśv II, 17, 15; V, 5, 18.

${ }^{639}$ RV V, 54, 1. - Bloomfleld hat in der Vedic Concordance irrig svabhänavah statt suabhänave. - Diese beiden Spriche sind nach der Pray. anuvälyä und yäjya für die Maruts; für Indra werden dort andere Sprùche aufgeführt.

aso So durch den Komm. verdentlicht.

o11 S. ZMIR Anm. 95.

${ }_{612}$ S. Anm, 640.

o43 Komm.

ous Komm,: von König und Untertanen (viś).

als Komm. 
19. „Her zu unserem, o Indra-Bṛhaspati... $\ll^{646}$ (und) »Für uns, o Indra-Ḅ̣haspati... $\ll^{647}$ (sind die Sprüche), wenn auch (die adhvary $u^{048}$-Priester ${ }^{649}$ allein) für Indra aufrufen.

Ende der 11. kaụdikā

II, 12

1. Beim Läuterungsopfer - 050

Ende der 12. kạ̣dikā

II, 13

1. Das Opfer eincs, der Regen wünscht ${ }^{651}$, (heißt) kärärï (-Opfer).

2. In diesem sind $" \mathrm{Z} n$ diesem wohlgefälligen Opferfest . . «\$52 (und) "Ich preise Agni, den guten Schutz Gewährenden, mit Verneigungen « ${ }^{653}$ die beiden dhäyyās ${ }^{654}$.

3. Bei einem jeglichen Regenopfer (enthalten) die beiden (äjyabhägaanuväkyās ${ }^{655}$ die Worte) sin den Wassern«:

4. "In den Wassern, o Agni, ist dein Sitz« ${ }^{656}$ (und) $» I n$ den Wassern, hat mir Soma erzählt . . « $\ll^{057}$

5. Agni der (seine) Wohnstatt Verhüllende, die Maruts (und) Sūrya (sind die Gottheiten) ${ }^{658}$.

6ะ6 RV IV, 49, 3. Es handelt sich um die anuvākyā.

${ }^{617}$ RV IV, 49, 4. Es handelt sich um die yäjyä.

${ }^{648}$ Die Gruppe der die eigentliche Opferhandlung ausfithrenden und liturgiseh mit dem Yajurveda arbeitenden Priester.

"49 So der Komm.

650 Dieses Opfer wird im Originaltext nicht weiter behandelt, wohl aber in der Pray. Es ist ein Entsthmungsritus für mehrere Generationen, der auch die Wiedergeburt vermeiden läßt. Ausführlichste Quelle - auch fiur die von hotar zu rezitierenden Sprïche - ist BaudhŚS XXVIII, 2.

651 Es handelt sich also um einen Regenzauber.

652 RV I, 19, 1.

6s3 R.V V, 60, 1.

654 Die in die sämidhenis eingeschobenen Verse; s. II, 1, 26.33.

655 Pray.

${ }^{656}$ RV VIII, 43, 9; vgl. Assv II, 13, 12 ,

657 R.V I, 23, 20; X, 9, 6.

658 Pray.: oder ersterer allein. 
6. Und als nächste (werden) drei Mehlklösse ${ }^{659}$ (geopfert) ${ }^{660}$.

7. Die mit $»$ Der Goldhaarige inmitten der Atmosphäre . . «661 begimnenden beiden Verse ${ }^{662} \ldots{ }^{663}-1$ Du selbst unerschütterliche ... « ${ }^{664}$ (und) $»$ Auf deiner Stäte beruht die ganze Welt $\ll^{665} \ldots{ }^{666}$. $-»$ Wie eine Kuh den Blitz anbrüllt . . « ${ }^{667}$ (und) "Wenn auch mächtig und groß, hat das Gebirge sich gefürchtet ${ }^{668} \ldots{ }^{669}-»$ Sie entsenden den Strahl mit Macht ${ }^{670}$ (und) "Mit den auf's Beste ziehenden (Rossen) kommst du, ausspannend das Gewebe $\ll^{671} \ldots$. $^{672}$. — $\gg$ Maruts, vom Meer her erhebt ihr euch «673 (und) "Voran, o Maruts, Kräftige, Wasser Ausströmende, euer ... «674 $\ldots{ }^{675}$. - $\gg$ Herwärts die Helden, die schön Träufelnden, dem, der verehrt hat $\ldots{ }^{670}$ (und) $\gg$ Die funkelnden Glanz habenden Helden, deren Geschosse Steine sind . . ${ }^{677} \ldots{ }^{678}$. - Schwarz sind die abwärts (führenden) Wege, feuerfarben die (göttlichen) Rosse «"79 (und) »Die mit cinem Vielgespann

659 Komm. und Pray.; den Maruts.

${ }^{680}$ Dies ist nur cine Zwischenbemerkung, da die Obliegenheiten des adhvaryu im hautra naturgemäß nur gestreift werden.

66: RV I, 79, 1.

${ }^{6 \varepsilon 2}$ Also RV I, 79, 1-2.

${ }^{603}$ Diese sind nach dem Komm. dann zu gebrauchen, wemn Agni allein die Gottheit ist.

664 KV VI, 2, 9. Slatt tvantyä ist besser toam tyā zt lesen.

665 RV IV, 58, 11.

680 Diese sind nach dem Komm. anuväkyā und yäjyä, wenn Agní Dhämacchad die Gotheit ist.

$667 \mathrm{RV} \mathrm{I}, 38,8$.

668 RV V, 60, 3.

${ }^{669}$ Diese beiden Verse sind an die Maruts gerichtet.

870 RV VIII, 7, 8 .

o7. RV IV, 13, 4.

67a Diese beiden Verse sind an Sŭrya gerichtet.

673 RV V, 55, 5 .

674 RV V, 54, 2.

${ }^{675}$ Diese beiden Verse sind naclı der Pray, anuvākyā und yäjyã zum ersten Klößcopfer.

${ }^{620} \mathrm{RV} \mathrm{V}, 53,6$.

677 RV V, 54, 3.

678 Verse zum zweiten KlöBcopter.

$679 \mathrm{RV} \mathrm{I}, 164,47$. 
Versehenen, wie Helden einen Trupp Besiegenden . . « ${ }^{680}$ ...681

8. $„ \mathrm{O}$ Agni, treibe hinweg die Feinde, hinweģ die Gefahren ${ }^{082}$ (und) $»$ Den dich Devăpi, der ausgezeichmete, o Agni . . « ${ }^{683}$ sind die beiden samyajyjass. - Einige (Lehrer) opfern, nachdem (lie Verse rezitier ${ }^{684}$ wurden, mit yajus (-Sprïchen) ${ }^{685}$.

9. Wenn (das Opfer) vollendet ist, verehre er alle Himmelsrichtungen mit den mit $" Z u$ (lem Mächtigen ${ }^{680}$ rede mit diesen Gesängen $\aleph^{887}$ (beginnenden) vier (Versen) oder jede einzelne Himmelsrichtung mit einer ganzen Hymne ${ }^{688}$.

\section{Ende der 13. kạ̣ḍikä}

II, 14

1. Hierauf (werden) die ayan $a^{659}$ (genamten) iștis ${ }^{690}$ (besprochen), -

2. (zunächst) die, die sich (auf den Ablauf eines ganzen) Jahres beziehen.

3. Deren Durchführung (beginnt) zum Vollmond des (Monats) Phälgun ${ }^{601}$ oder (des Monats) Caitra ${ }^{692}$.

680 RV V $54,8$.

681 Verse zum dritlen Klößcopfer.

${ }_{682} \mathrm{RV} \mathrm{X}, 98,12$. Statt durgrahä ist durgaha zu lesen.

${ }_{683} \mathrm{RY} \mathrm{X}, 98,8$.

${ }^{681}$ Als anuväkya (Komm.).

${ }_{685}$ Die somit die Stelle der yajyäs einnehmen wïrden. Fitr einen hotar ist eine solche Verfahrensweise jedoch ungebräuchlich.

686 Parjanya, dem Gott des Regens.

B87 RV V, 83, 1.

Q88 Diese Alternallve wurde nach dem Komm, gedleutet. - Hiermit schließt. die Besprechung der eigentlichen Wunschopfer.

oso = Gang, Weg.

690 Es handell sich hier also um ayanas von iş/is, während die bekanntesten ayanas, etwa das gavām ayana, dem Somaritual angehören. Im Folgenden werden die in irgendeiner Form das darsapürnanäsa-Ritual ergänzenten ayanas behandelt. Einfliisse des Somakultes zeigt etwa sütra 5.

601 Name eines Monats, galt als Beginn des Fruhlings und damit des Jahres; vgl. TS VII, 4, 8, 1-2; KB IV, 4; V, 1 ; JB I, 81; TB I, 1, 2, 8. 4, 7, 5-6; PB V, $9,7-9 ;$ SB IV, $5,10,2 ; \mathrm{VI}, 2,2,18$.

602 Nane eines Frühlingsmonats, lag später als der in Anm. 691 genannte (März-April); vgl. KB XIX, 3; TB III, 1, 4, 12. 
4. (Nun folgi) der luräyana (-Ritus).

5. Agni, Indra (und) die Allgötter (sind die Gotheiten). Die Opfer (erfolgen) einzeln entsprechend der Somapressung 603 , 'Tag für Tag.

6. Oder einmal ${ }^{694}$ mit drei Opfergaben.

7. Beim däkșäyana (-Ritus) ${ }^{695}$ bringe man zwei Vollmond- (und) zwei Neumondopfer dar.

8. Beim ersten (der beiden Vollmondopfer gelten) die üblichen beiden (Gollheiten) ${ }^{696}$, beim (ersten der beiden) Neumondopfer (diejenigen) wie bei einem, der keinen sämnäyya $a^{697}$ darbringt ${ }^{698}$.

9. Von den beiden Zweit (-opfern) gehört Indra am Vollmondstag die zweile (Spende), -

10. Mitra-Yarupa am Neumondstag.

11. "(Mögen) Mitra-Varuna unsere ... «"99; ;Den festesten (Schutz), nicht zu durchbohren, o ihr beiden schön 'Гräufelnden ... $\ll^{700}$ - Prajäpation zugehörig ist das ịlädadha (-Opfer).

12. „O Prajāpati, kein anderer als du (hält) diese ... «" ${ }^{702}$, Dir (gehören) diese Welten, die Weltgegenden und die Himmelsrichtungen ${ }^{703}$, die Fernen, die Unter- und die Himmelswelten $^{704}$. O Prajāpati, Allschöpfer (bist du), den Lebe-

${ }_{\text {B93 }}$ D. h. dreimal tiglich. Pray.: morgens für Agni, miltags für Indra, nachmittags für die Allgötter.

oof Komm.: in diesen Falle morgens.

695 Er dient mach $\AA_{p}$ III, 17, 4 zur Erreichung des Himmels und ist also eine kaimyești.

696 Pray.: Agni und Agni-Soma.

697 Eine Mischung aus süßer und saurer Milch; vgl. TS II, 5, 3, 3. 5, 1; VII, 5, 6, 4; АВ VII, 2.4; PB XXV, 10, 3; 'TB III, 2, 3, 11; SB I, 6, 2, 6; II, 4, 4, 8.20; $X I, 1,7,2.2,6,6 ; A p ~ I, 6,13.11,4 ; 11,19,1$; Uăty IV, 2, 33.

008 Pray.: Agni und Indra-Agni.

soo RV III, 62, 16; vgl. ASV V, 10, 28; VII, 2, 2. 5, 9. Dieser und der folgende Vers sind anuvākya und yäjyã für Mitra-Varmua.

$200 \mathrm{RV} \mathrm{V}, 62,9 ;$ vgl, Xsv III, 8, 1.

301 Vor diesem Wort lätte besser eine sütra-Trennung erfolgen sollen.

${ }_{702} \mathrm{RV} \mathrm{X}, 121,10$; vgl. Aśv III, 10, 23. Dieser Vers ist die anubäkya.

${ }^{203}$ Vgl. MS IV, 14, 1; 'ТВ II, 8, 1, 3; $\AA$ śv X, 9, 5; $\mathrm{p}_{\mathrm{p}} \mathrm{XX}, 20,9$.

704 Vgl. TB II, 8, 1, 4 einschl. des dorligen Kommentars; sonst wie in Anm. 703 ohme Asv X. 
wesen Schätze bringend ${ }^{705}$. Diese unsere Opfergabe, o Gott, nimm entgegen! $\ll^{706}$ - (Dieses Opfer heißt auch) der Weg von Himmel und Erde.

13. Mit den Vollmondopfer (opfere man täglich ${ }^{707}$ ) bis zum Neumondstag, mit dem Neumondopfer bis zum Vollmondslag.

14. Bei den (hier) nicht aufgezählten (iș is tritt) wegen des (veränderten) Zweckes eine Veränderung der Grundregel ${ }^{708}$ ein.

15. Wie der adhvaryu $u^{709}$ fürwahr verfahre man ${ }^{710}$.

16. Das viräj-Metrum aber (herrscht bei denjenigen $i s ̦\left(i \mathrm{~s}^{711}\right.$ ), wo Feuer durch Bohren (erzengt wird).

17. Nur die beiden dhăyyäs (Zusalzverse) (stehen im viräjMetrum, meinen) aber einige (Lehrer).

18. Die die Charakterisierung von Gottheiten enthaltenden (Verse ${ }^{712}$ heißen) anuvälyya und yäjyä.

19. Der in gãyalrit-Metrum ${ }^{713}$ stehende, (die Worte) wherbei « ${ }^{714}$, "gerufen « ${ }^{715}$ (und) »aufgerufen « ${ }^{716}$ (und) das Merkmal (der Gottheit) in der crsten Hälfte ${ }^{717}$ enthaltende (Vers heißt) anuāakyă.

20. Der im triștubh-Metrum ${ }^{718}$ stehende, (die Worte) »erquickt «119 (und) »erfreut« ${ }^{720}$ (und) das Merkmal (der Gottheit) in der

705 S. Amm. 704.

700 S. Ann. 704.

707 Nach dem Komm., der hinzufilgt, daß man so ein Jahr lang verfahren mïsse. - Im Text muB es paurnamāsena statt pärṇamāsena helßen.

${ }^{708}$ ISomm.: sie weicht dann vom Volmondopfer ab.

700 S. Anm. 648 zu II, 11, 19.

30 D. l. man folge dem Y'ajurveda (Komm.).

711 Komm. - Man beachte, daß vi-rajj auch strahleno bedeutet.

712 Komm.: $r k s ̧ t$, also Verse aus dem RV. Vgl. hierzu Anm. 685.

${ }^{713}$ Die gâyalri besteht aus $3 \times 8$ Silben mit einer Zäsur nach den ersten beiden päalas.

$714 \ddot{a}$.

715 hüla.

716 upokla.

717 Komm.

718 Die triș/ubh besteht aus $4 \times 11$ Silben mit einer Zäsur nach den ersten beiden pădas.

719 vila.

7 a jușla. 
zweiten Hälfte enthaltende (Vers heißt) yäjya. (Anuwäkya und $y \bar{a} j y a ̄ a ̈$ könen) auch einem anderen Metrum angehören.

21. Aber die yājya ist nie kürzer (als die antwākyā).

22. Weder die uṣnihih ${ }^{721}$ noch die bḷhali ${ }^{722}$ (kommen als Metren für dic yājya in Betracht).

23. (Verse, die die Worte) "angebrannt ${ }^{723}$, "umgekommen « ${ }^{724}$, "getötet «25 (oder) "verbrannt ${ }^{726}$ (enthalten), vermeide man aber ${ }^{72 ?}$.

24. Ist nur das zu einer Gottheit gehörende (Kennzeichen) oflenkundig, (verfahre man) ebenso ${ }^{728}$.

25. Oder auch beim Fehlen eines solchen Kennzeichens ${ }^{729}$.

26. Findet man keine (entsprechenden Verse, berïcksichtige man den Veda) insgesamt.

27. Findet man (auch da) keine (passenden, opfere man) mit solchen, die zu Agni gehören.

28. Oder mit den vyahṛtis ${ }^{730}$.

29. Nachdem man die Gotheil genannt hat ${ }^{731}$, murmele ${ }^{732}$ und opfere man.

30. Oder (man verwende ${ }^{733}$ ) die beiden $» V e r n e i g u n g s v e r s e \aleph^{734}$.

31. "Höre auf (liesen Ruf ${ }^{735}$ ) mit Liedern rufen wir dich ${ }^{730}$. Auf dieser unserer Opferstreu la $\beta$ dich nieder ${ }^{737} 1$ « (Und:)

721 Die uşuih besteht aus $2 \times 8+1 \times 12$ Silben.

${ }^{22}$ Die brhati besteht aus $2 \times 8+1 \times 12+1 \times 8$ Stlben.

$72 s$ kșāmu.

724 nașla.

725 hata.

226 dagdha.

${ }^{227} \mathrm{Komm} .:$ in antwäkyäs und yajyās.

728 D. h. man verwende einen solchen Vers wic oben.

${ }^{720}$ Komm.: damn sind das gäyatri-Metrum und die anderen Charakteristika zt berücksichtigen.

730 S. ZMR Amm, 414.

731 Komm.: im zweiten Kasus.

${ }^{92}$ Komm.: bhär bluvah svar om. - Dies beim Gebrauch als anuväkyã. Für de Verwendung als yäjua gibt der Komm, weitere Anweisungen.

${ }^{233}$ Als anuväkyā und yäjyä.

734 Der Komm. fuhrt allerdings nämra auf nämadheya zurỉck statt auf die Wz. nam.

735 Vgl, Sänkh I, 17, 19.

730 Vgl. Sājkh I, 17, 19.

737 Variiert aus RV II, 41, 13; VI, 52, 7.

10 Acta Orientalia, XXXIV 
»Begebt euch der Reihe nach auf die ausgebreitete Opferstreu $^{738}$. Zu uns, die wir (dich) hier anflehen, komm heute ${ }^{739}$. Mit nicht zürnendem Geist erfreue dich an dieser (Opfergabe $)^{740}$. Empfange meine Opfergabe, die dargebotene, geopferte ${ }^{741}$. (Dies sind) dic beiden $" V$ erneigungsverse ${ }^{742}$.

32. (Wenn auch diese) beiden Verse keine (Gottheit) bezeichnen, gehören sie (doch) zu Agni.

Ende der 14. kaṇḍikā

\section{II, 15}

1. Wer die Tertialopfer ${ }^{743}$ durchführen will, (begimne) am Tage zuvor mit einem (Opfer) an (Agni) Vaiśvānara und Parjanya.

2. "Agni Vaiśvānara schuf ${ }^{744}$ uns einen ncuen Heilsgedanken ${ }^{745}$, auf der Erde wachsend mit Macht ${ }^{246}$. Begehrt im Himmel, begehrt (ist) Agni auf der Erde ${ }^{74}$. Dem Parjanya singel! ${ }^{78}$ Vorwärts die Winde wehen, es fallen die Blitze ${ }^{749} .4$ - Vom agnyädheya an bis zu diesem (Opfer erfolgen) dic Opfergaben als upāinśu ${ }^{750}$.

3. (So ${ }^{751}$ auch) bei don zum Somaritual gehörenden (ișlis).

${ }^{798} \mathrm{~S}$. Amm. 735. Es ist nicht klar, ob a sadeta upedănã oder asad etad uped̦anā zu lesen ist. Auffallend ist auch das änușak, das dem sonst durchgehenden Imper. Sg. widerspricht.

739 S. Anm. 735.

740 S. Anm. 735.

741 S. Anm. 735.

${ }_{733}$ Der Inhalt dieser mantras zeigt also dentlich, daB sie im Tone demutsvoller Verneigung gehalten sind und mit Namengebung nichts zu tun haben.

${ }^{733}$ călurmāsyāni. - Nachidem von Ende der 9. kauḍikã an hauptsächlich kleinere, meist aperiodische Riten besprochen wurden, folgt num bis zum Schluß des II. adhyāya das hautra eines sehr bedeutsamen haviryajna; vgl. A. Hillebrandl, Ritual-Litteratur, S. 115-119.

${ }_{744}$ Vgl. AB V, 17, 13; VII, 9, 1; ISB XXVI, 13; Xsv VIII, 9, 7; Sänikh X, $10,8$.

745 Vgl. Śānkh X, 10,8.

746 Vgl. VS XXXIII, 92; KB und Sánklı wie in Anm. 744. - Bis hier geht die anuvăkyã.

${ }_{747}$ RV I, 98, 2. Dies ist die yäjyă.

$7 * 8$ RV VII, 102, 1. Dies ist die antuākyā fiùr Parjanya,

$738 \mathrm{KV}$ V, 83, 4. Dies ist die yajjyā für Parjanya.

750 D. h. die Rezitationen erfolgen mit leiser Stimme.

751 Nämlich ats upāinsus. 
4. (Ebenso) bei den zum Sühmeritual gehörenden (iștis).

5. (Ebenso beim Vorhandensein) zusätzlicher (Gottheiten sowie bei Opfern) auf éiner Tonschüssel.

6. Durchweg (sind Opfer) an Varupa ausgenommen.

7. (Opfer) an Savitar bei den Tertialopfern (erfolgen als upāmśu $\left.{ }^{752}\right)$.

8. Die Hauptspenden ${ }^{763}$ auch, (sagen) einige (Lchrer).

9. Die Manenopferdienste erfolgen (ebenfalls) in dieser Art, -

10. die auf die Wiederanlegung der heiligen Feuer ${ }^{754}$ bezüglichen (erfolgen als upāmśu) bis hin zum letzten Nachopfer ${ }^{755}$ (-sprueh).

11. Oder (alle ${ }^{750}$ diese Stellen können) auch im wohllautenden Tiefton (ausgeführt werden).

12. Der ăgur-Ruf ${ }^{757}$, das $O m$ und der voșat-Ruf (erfolgen) allenthalben mit lauter (Stimme).

13. So (auch) beim Feldfrucht-Erstlingsopfer zu Beginn ${ }^{758}$.

14. Das $O m$ ist mit der puronubāky $\bar{a}^{759} \mathrm{zu}$ verbinden und in éinem Atem (zu rezilieren).

15. So (geschieht auch die Verbindung von) ägur- und vașațRư mit der yājyã.

16. Die lauten (Wörter ${ }^{760}$ ) in einem upämśu-Ritus (haben) die Tonhöhe der (diesen Ritus betreffenden) Sprüche.

17. In den upāmśu-Riten (sind die laut zu sprechenden Wörter ${ }^{761}$ ) in tiefer Tonlage (zu sprechen) ${ }^{762}$.

Ende der 15. kạ̣ḍikã

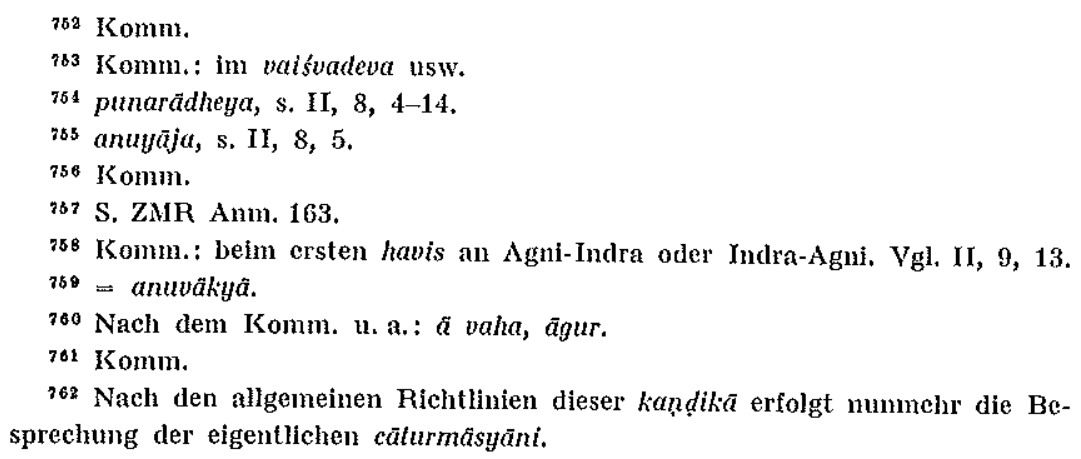


II, 16

1. Morgens, wenn das vaiśvadeva-O pfer ${ }^{783}$ dargebracht wird, soll $e^{764}$, nachdem er die Feuerbohrverse hergesagt, sich in der Entfernung eines Fußes westlich des Platzes (zum Rezitieren) der sämidhenti. Verse ${ }^{765}$ aufgestellt und him gesagt hat, (rezitieren :)

2. "Zu dir, o Golt Savitar ... «706; »Die beiden Mächtigen, Himmel und Erde, uns . . $\ll^{767}$ und die drei mit $»$ Dich, o Agni, von der Lotusblüle her . . « ${ }^{768}$ beginnenden (Verse). Nachdem er den Halbvers ${ }^{769}$ rezitiert hat, halte er inne bis zu einer besonderen Aufforderung ${ }^{770}$ (zum Fortfahren).

3. Ist sonst am Ende des (Halb-)verses ein Anhalten bewirkt, (verharre er) bei diesem Ruhepunkt.

4. „O Agni, du schlägst die Verschlinger nieder«"7n; (diese) Hymne füge er immer und immer wieder ein bis zum Entstehen (des Feuers).

5. Wenn er gehört hat, daß es entstanden ist, fahre er alsbald mit dem Om-Laut in der Rezitation fort.

6. Wenn er es während der Rezitation (erfährt, schlage er die letzte Vershälfte) dem folgenden (Vers ${ }^{772} \mathrm{zu}$ ).

7. "Und die Menschen sollen sprechen « ${ }^{773} ; "$ Den an der Hand wie eine Spange . . « (774 $^{7}$ nachdem er (hiervon) den Halbvers (gesprochen hat), halte er an. (Dam spreche er) die mit "Vorwärts den Gott zum Göttermahl«775 beginnenden beiden

703 Das erste Tertialopfer.

281 Der holar.

265 S. $1,2,7$.

768 RV I, 24, 3; vgl. Asv IV, 7, 4; V, 12, 9; VIII, 9, 5.

${ }^{767}$ S. Anm, 513 zu II, 9, 14 .

$76 \mathrm{~g}$ RV VI, 16, 13.

769 Komm.: den (ersten) Halbvers des letzten der mit toām agne begimnenden drei Verse.

"7o sampraișa; geht gewöhnlich vom adhuaryu aus; vgl. Āsv III, 2, 2; Kāty

I, 3, 11.

77 RV X, 118, 1; vgl. Asv IV, 13, 7; VIII, 12, 7.

778 Komm.

73 LV 1, 74, 3; vgl, Asv II, 18, 15.

774 RV VI, 16, 40.

775 RV VI, 16, 41-42. 
(Verse). Mit $»$ Durch Agni wird Agni entlammt ${ }^{726} ; "$ Denn du, o Agni, durch Agni ... «"n7; "Den Weisen schmücken sie ${ }^{778}$; "Durch das Opfer opferten die Götter das Opfer « ${ }^{779}$ vollendet ${ }^{780} \mathrm{er}$ (die Rezitation).

8. Überall kenne man den letzten (Vers) unter der Bezeichnung paridhănüyă ${ }^{981}$.

9. Die beiden eingeschobenen Verse (stehen) im viraj-Metrum. (Es gibt) neun prayäjas ${ }^{782}$. Vor dem letzten schiebe er die vier (Sprïche) ein: »Es sollen die Tore, o Agni, vom Opferschmalz annehmen ${ }^{783}$; es sollen Morgenröte und Nacht, o Agni, vom Opferschmalz annehmen ${ }^{784}$; es sollen die beiden göttlichen hotars, o Agni, vom Opferschmalz annehmen ${ }^{785}$; es sollen die drei Göttinnen, o Agni, vom Opferschmalz anmehmen ${ }^{786}$ «

10. (Die Gotheiten sind:) Agni, Soma, Savitar, Sarasvatī, Pūșan, die Selbststarken Maruts, die Allgötter (und) Dyãvāpṛthivĩ.

11. "Den Allgott, den Herrn des Guten ....4787; "Wohlstand heute, o Savitar, Wohlstand morgen . . « ${ }^{788}$ (sind die Verse an Savitar). "O Pūṣan, unter deiner Obhut wir ...«" "Hell ist deine eine (Form), verehrungswïrdig deine andere ${ }^{790}$ (sind die Verse an Pūșan). »Hier und dort, o ihr Selbststarken ....4701; "Ein prächtiges Preislied dem singenden, ungestïmen ... $\ll^{792}$ (sind die Verse an clie Maruts).

778 RV I, 12, 6; Ygl, Aśv III, 13, 12.

"7R RY VIII, 43, 14; vgl. Xsv III, 13, 12.

${ }^{738}$ RY VIII, 84, 8. Es ist tam marjayanta statt tamarjayanta zu lesen.

$779 \mathrm{XPV}$ I, 164, 50; X, 90, 16.

${ }^{280}$ So umschreibt der Komm, paridadhyät.

781 Vgl. $\lambda s_{v} V, 1,1.14,24 ; A p$ II, 12, 6.

782 Voropfer-Sprüche; s. Amm. 452 zu II, 8, 5.

${ }^{783}$ Vgl. MS IV, 10, 3; KS XX, 15; S̄̄nkh III, 13, 20.

784 S. $\Lambda$ m. 783.

785 S. Anm. 783.

${ }^{286}$ S. Amm. 783.

${ }^{787}$ RV V, 82, 7; vgl. Aśv IV, 3, 2. 11, 6; VII, 6, 6,

788 RQV VI, 71, 6.

$789 \mathrm{RV}$ VI, 54, 9.

790 RiV VI, 58, 1; vgl. Ås III, 7, 8; IV, 6,3 .

$721 \mathrm{RV}$ VII, 59, 11.

792 RV VI, 66, 9; vgl. Aśv III, 7, 12. 
12. (Es gibt) neun anūyäjas ${ }^{793}$. Nach dem ersten (rezitiere er die folgenden) sechs: "Die götllichen Tore sollen zur Reichtumsgewährung die Reichtumsgabe annehmen. ${ }^{794}$ Die göttliche Morgenröte und Nacht sollen zur Reichtumsgewährung die Reichtumsgabe annehmen ${ }^{795}$. Die göllliche Begehrerin soll zur Reichtumsgewährung die Reichtumsgabe annehmen ${ }^{796}$. Die göttliche Nahrung und Opferspende sollen zur Reichtumsgewährung die Reichtumsgabe annehmen. ${ }^{797}$ Die Götter, die beiden götllichen hotars, sollen zur Reichtums gewährung die Reichtumsgabe annehmen ${ }^{798}$. Die Göttimnen drei, die drei Göllinnen sollen zur Reichtumsgewährung die Reichtumsgabe annehmen ${ }^{799}$."

13. Nach den anūyăjas bzw, dem sūktavāka $a^{800}$ oder dem samyu$v a ̈ k a^{801}$ (vollziehe er) für die Väjins, die er (vorher) nicht eingeladen hat, die väjina-Aufforderung ${ }^{802}$ :

14. "Segen sollen uns die Văjins sein bei den Anrufungen ${ }^{803}$; In cinem jeden Kampf helft, o Väjins, uns! ${ }^{804}$ - Mit aufwärts gerichteten Knien, ohne Atem zu holen, (rezitiert er) die yăjyă.

15. »O Agni, nimm an . . « ${ }^{805}$ (ist) die anuvașa ${ }^{806}$-Formel oder „Die Molkenspeise, o Agni, nimm an! ${ }^{807}$ Wo bei einer Auf-

${ }^{293}$ Nachopfer-Spritiche, s. Anm, 453 zu II, 8, 5.

701 Vgl. MS IV, 10, 3; IV, 13, 8; IS XX, 15; TB III, 6, 14, 1; Şảnkh III, 13, 27.

795 S. Anm. 794 auBer MS IV, 13, 8.

208 S. Anm. 794.

797 S. Anm. 795.

708 S. Anm. 794.

709 S. Anm. 795.

800 S. ZMR Anm. 291.

801 S. ZMR AHm. 191.

${ }^{802} \mathrm{v}$, ist eine Hischung aus heißer, frischer, süßer Milch mit saurer; vgl, TS I, 6, 3, 10; VS XIX, 21.23; VSK XXI, 2, 10.12; AB I, 13. 22; TB I, 6, 2, 5; SB II, 4, 4, 21-22; III, 3, 3, 2; IX, 5, 1, 57; \$BK I, 3, 4, 12-13; Ap VIII, 2, 1.6.

803 RV VII, 38, 7.

804 RV VII, 38, 8.

${ }_{803}$ Vgl. AB I, 22, 4-5; ŚB II, 4, 4, 23; ג约 III, 9, 4; IV, 7, 4; V, 13, 6; Mãn $\mathrm{V}, 1,3,11$.

${ }^{800}$ S. Āp VIII, 3, 8.10. Es handelt sich um eine das svistakpt-Opfer begleitende Formel.

${ }^{807}$ Vgl, Săảnkh III, 8, 24; Åp VIII, 3, 10. 
forderung zwei vaşal-Rufe sind, dort spreche er über diese zusammen zweimal die Schlußfomel.

16. Nicht (erfolgt) die ägur-Formel beim zweiten (vașal-Ruf).

17. Nachdem er die Molkenspeise wie eine ị̣ă $\ddot{c}^{808}$ ergriflen hat, wünsche er die Einladung (mit:)

18. $" \mathrm{O}$ adhvaryu, lade ein $!^{809} ;$ o brahman ${ }^{810}$, lade ein! ${ }^{811} ; 0$ agnīlh ${ }^{812}$, lade ein $\left.\right|^{813}$

19. "Mit dem Samen, der mir vergossen wird ${ }^{814}$, oder der in mich eingeht ${ }^{815}$ oder der wieder entsteht $t^{816}$, damit tritt in mich zum Heile ein ${ }^{81 ?}$, damit mache mich zur Molkenspeise! ${ }^{818}$ Von dir, dem von den Vājins getrunkenen, eingeladenen genieße ich als Eingeladener ${ }^{819}$." (Damit) genieße er eine Atemspeise ${ }^{820}$.

20. Ebenso $^{821}$ (tum es) adhvaryu, brahman ${ }^{822}$, agnìdhra ${ }^{823}$.

21. Der Opferveranstalter (aber ißt) ausdrücklich, und (ebenso) andere (zam sattra ${ }^{824}$ ) Geweihte.

808 S. ZMR Anm, 242 ,

${ }^{800} \mathrm{Vgl.} \mathrm{ȘB} \mathrm{II,} \mathrm{5.7;} \mathrm{SB} \mathrm{IV,} \mathrm{4,} \mathrm{2,} \mathrm{16;} \mathrm{Āsv} \mathrm{V,} \mathrm{6,} \mathrm{2.14.}$

810 Der vierte mahartvij, der die Opferhandlung zu ïberwachen und Ritualfehler zeremonfell zu sühnen hat.

811 Vgl. ŞB II, 5-7.

${ }^{812}$ Identlsch mit ägnïlhra, nach der Rilualtheorie Gehilfe des brahman, in Whklichkeit des adlwaryu, ist mit dem Anzinden des Opferfeuers beanftragt; vgl. TS VI, 5, 8, 5; VS VII, 15; AB V, 25; IKB X, 3; XXX, 1; PB XVIII, 9, 14; $\mathrm{XX}, 15,14$; XXV, 15, 3. 18, 4; TB I, $1,6,9.8,2,5$; III, 3, 7, 3. 8, 1.8.11. 7, 2, 6; SB I, 2, 4, 13.18; SBIK I, 3, 4, 14; GB II, 2, 9.16. 3, 18. 4, 5; 'TA II, 2, 1. 3, 1. 5,$1 ; \mathrm{IV}, 4,1 ; \mathrm{X}, 64,1$.

813 Erscheint nur hier.

814 Vgl. TA I, 30, 1; Śảnh III, 8, 27; Lāṭ IV, 12, 16; Vait VIlI, 16.

815 S. Anm. 814 ohne TA und Sāilkh.

816 S. Anm. 815.

812 S. Alum. 815.

818 S. Anm. 815.

819 S. Amm. 815.

820 D. h. ex rieche nur daran.

821 Komm, : die Atemspeise genieBen.

822 S. Anm. 810 zu II, 16, 18.

823 S. Anm, 812.

${ }^{824}$ Komm. - Der Begrifi sattra wird bei der Behandlung des Somarituals erläutert werden. 
22. Nachdem er ein Vollmondopfer dargebracht hat, unterziehe er sich den Tertialopfer-Gelübden.

23. Die Kopfhaare beseitige er.

24. Die Barthatre lasse er abrasieren. Er schlafe auf der Erde. Er meide Honig, Fleisch, Salz, Weib (und) Haarkämmen.

25. Der Gattin möge er sich während der Menses nähern.

26. Das Rasieren (erfolgt) bei sämtlichen Einzel-Terlialopfern ${ }^{825}$.

27. Oder nur beim ersten und beim letzten.

Ende der 16. kaṇḍikã

II, 17

1. Am fünften Vollmondstag (feiert man) mit den Varunapraghäsa $^{826}$-Riten.

2. Nachdem er ${ }^{827}$ sich westlich von dem nach Art des Neuund Vollmondopfers (errichteten) Altars niedergelassen hat, respondiere er nach Aufforderung mit den (Versen) zum Vorantragen des Feuers:

3. (Zunächst) die drei mit »Vorwärts den Gott mit göttlicher Andacht ${ }^{828}$ (beginnenden Verse, damn) $»$ Dich an der Labetrunkergießung Stätte wir ... « ${ }^{829}$. Am Halbvers von $» O$ Agni, schön Aussehender, mit allen Göttern « ${ }^{830}$ verharre er. Als Sitzender rezitiert er den ersten (Vers), leise, mit dem Om-Laut.

4. Wo ein Übergang von einer Stimmlage ${ }^{831}$ in eine (andere) Stimmlage (erfolgt), beende er (den Vers) mit $\mathrm{Om}$ und rezitiere den nächsten, ohne Luft zu holen.

5. So ist ununterbrochen Atem vorhanden, das ist bekannt.

6. Dem Feuer nach Norden nachschreitend, (rezitiere er) die anderen (Verse).

825 Von denen das vorstehend behandelle vaisuadeva das crste ist.

8a Name des zweiten parvan der Tertialopfer.

827 Der hotar.

s98 R.V X, 176, 2.

820 RV III, 29, 4.

830 RV VI, 15, 16. - Es ist iḍăyãs tvã pade vayam statt iḍayāstoāpa deva yamagne za tremmen.

B31 sthāna. Aufstellungen derselben geben Assv I, 5, 25; IV, 13, 6. 15, 10; Sănikh

I, 14, 22; $\bar{A}_{P} \times, 4,11 ; \times X I V, 1,12$. 
7. »Dem Mächtigen, in der Versammlung Hervorragenden, dieses Preislied ... (832 $^{83 z w .)} » D i e s e r$ (Agni) hier wird als erster eingesetzt von den (das Opfer) anordnenden (Priestern) $\varkappa^{833}$ aber ist jeweils der erste (Ver's) für cinen räjanya ${ }^{834}$ bzw, einen vaiśya $a^{835}$.

8. Westlich des Hochaltars ${ }^{830}$ sich aufhaltend, (vollende er die Rezitation ${ }^{837}$ ).

9. (Westlich) des Hochaltars auch bei Somaopfern.

10. Nachdem das Feuer niedergelegt wurde, (rezitiere er die) zwei (Verse ${ }^{838}$ ) ) "Setz dich, o hotar, an ehen deinen eigenen Platz, beobachtend $\wedge^{839}$ (und) "Nieder als hotar auf dem hotar-Sitz sich eingefunden habend ....(1840. Nachdem er den Schlußvers gesprochen hat, erhebe er, an eben derselben Stelle sitzend, die Stimme mit "bhūr bhwahl svaḥ «"1.

11. Bei anderer Gelegenheit möge er, während er rezitiert, (dem adhvaryu ${ }^{842}$ ) folgen.

12. Steht er bei den Aufforderungen, dann (erfolgt) $\mathrm{so}^{843}$ die Erhebung der Stimme.

13. Das Feuerbohren und die folgenden (Riten) sind mit dem vaiśvadeva-Ritual identisch.

14. An die Stelle (der Gotheiten) bei der sechsten und den folgenden havis-Spenden (treten jedoch) Indrāgnì, die Maruts, Varuṇa und $\mathrm{Ka}^{844}$.

15. "O Indra-Agni, kommt herbei mit Labung«815 (und) »Es

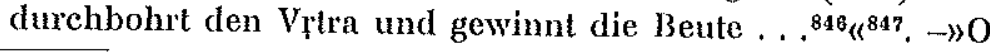

\footnotetext{
832 R.V III, 54, 1 .

${ }^{893}$ R V IV, 7, 1.

834 S. Allm. 4 zu II, 1, 3.

sas Angehöriger des dritten varna.

836 ultara vedi; vgl. Ap VII, 3, 13; XVI, 13, 11; SBE XII, S. 392.

837 Konmm.

${ }^{838}$ In den beiden folgenden Versen wird der Gott Agni als hotar bezeichnet.

839 RV III, $29,8$.

$840 \mathrm{RV}$ II, 9, 1.

841 Vgl. $\not$ śv II, 3, 27. 5, 13.

812 Dies geht aus dem sampraişa des folgenden sintra hervor.

843 Also stehend.

844 Wörtlich der oVer? ein Name des Schöpfergottes Prajāpati.

8 เి R.V VII, 94, 7; vgl. Áśv I, 6, 1.

${ }^{816}$ R.V VI, 60, 1 .
} 
Maruls, in wessen Wohnung . . $4^{848}$ (und) Wie Radspeichen, von denen keine die letzte ist, wie Tage . . ${ }^{84} \iota^{850}$. - Diesen meinen (Ruf), o Varuṇa, höre $«^{851}$ (und) »Darum gehe ich dich an, mit Gebet verehrend ${ }^{852}\left\langle^{853}\right.$. - ${ }^{8 \text { Mit welcher (Hilfe) }}$ steht er als Strahlender uns bei ${ }^{854}$ (und) $\gg$ Goldkcim ${ }^{855}$ entstand zu Anfang ${ }^{850} \ll^{857}$. -

Der pratiprasthatar ${ }^{858}$ ist bei der Molkenspeise der dritte.

16. Ist (die Opferhandlung) vollendet, begibt man sich zum Reinigungsbad ${ }^{859}$.

17. Dort wird die Badezeremonie ausgeführt oder nicht ausgeführt.

18. Diese werden wir später ${ }^{860}$ erlklären. Nach $^{861}$ zwei Monaten (vollziehe man) ein an Indra-Agni (gerichtetes) Tieropfer.

Ende der 17. kạ̣dikã

II, 18

1. Ëbensoviel ${ }^{862}$ danach $^{863}$ (erfolgen) die săkamedha $a^{864}$-Riten.

8\$7 Dies sind diese Verse für Indra-Agni.

848 S. Anm. 638 zu II, 11, 14.

${ }^{840}$ RV V $, 58,5$; vgl. Åsv III, 7, 12. Es ist ived acaramä stalt ivedán caramá zu lesen.

850 Dies sind die Verse für die Maruts.

851 RV I, 25, 19.

${ }^{832}$ RV I, 24, 11; vgl. Aśv III, 7, 15.

833 Dies sind die Verse für Varuna.

Bs4 R̦V IV, 31, 1; Vgl. Ảsv V, 16, 1; VII, 4, 2; VIII, 12, 18. 14, 18.

s5s hiranyagarbha, ebenfalls ein Aspekt des Gottes Prajāpati.

8s6 RV X, 121, 1; vgl. Ásv III, 8, 1.

857 Dies sind die Verse für die Aspekte des Prajāpati.

858 Name des ersten Gehilfen des adhvaryu; vgl. TS VI, 5, 3, 4; KS XII, 10; KIKS XLII, 5-6; MS I, 10, 13; IV, 9, 2; AB I, 29; VII, 1; PB XXV, 4, 5; TB I, 6, 5, 2; SB III, 5, 2, 2. 3, 13.22; XI, 7, 2, 6; SBK I, 5, 1, 17.26.30.34-35.38; GB I, 4, 5; TA IV, 4, 1; А̄p VII, 18, 1. 21, 3; XII, 3, 15. 21, 21; XV, 3, 11 .

${ }^{859}$ avabhrtha; vgl. TS II, $3,12,2$; III, $3,8,1$; KS XXI, 11; MS III, 4, 1; VS VIII, 27; AB I, 3; VII, 17; KB XIX, 3; PB IX, 5, 12. 9, 15; ȘB III, 1; JB II, 67; TB I, 6, 5, 5; SB II, 4, 3, 4; XIII, 3, 6, 5; GB I, 4, 7; AA V. 3, 2; TA II, 13, 1; CU III, 17, 5; Ap VIII, 7, 14; XIII, 19, 1; XIX, 4, 6; XX, 22, 5.

860 Asv VI, 13, $1 \mathrm{ff}$.

861 Komm.

862 Also zwel Monate.

${ }^{863}$ Nach dem genannten Tieropfer (Komm.).

865 Name des dritten parvan der Tertialopfer. 
2. Am Tage zuvor (erfolgen) drei iștis entsprechend der Somapressung $^{865}$.

3. Bei der ersten (iști ist) Agni Anïkavant (die Gottheit. Für diese rezitiert der hotar:) »Den (das Opfer zum) Antlitz habenden ${ }^{860}$, den Agni rufen wir mit Liedern ${ }^{807}$ « (und) $» \mathrm{Er}$ möge uns hinwegführen über die Feinde ${ }^{808}$; möge er sie mit dem Antlitz als Gabemreicher uns ... «869. Bei der zweiten (iști erscheinen) die beiden vrdhanvant $\left(-\overline{a j} y a b h a \overline{g a s}{ }^{870}\right)$. Die Marutal Sănitapanăl (sind die Gottheiten. Für diese rezitiert er:) $) \mathrm{O}$ ihr wärmcliebenden Maruts, diese Opferspeise $\ldots «^{871}$ (und) $» O$ Maruts, der uns heftig züint . . « ${ }^{872}$ Für die das Hausopfer darbringenden Maruts beginnt die folgende $^{873}$ iṣli mit den äjyabhăgas und endet mit der iça.

4. (Hier lauten die beiden Sprüche:) »Dic ihr an Hausopfer Anteil habt, kommt herbeil« ${ }^{874}$ (und) »In die Tiefe dringen eure Herrlichkeiten vor ${ }^{875}$.

5. Die beiden sainyäjyās sind "gedeihenbringend", stehen im viräj-Metrum und enthalten keinen nigada ${ }^{876}$.

6. (Letzteres ${ }^{877}$ gilt) auch (dann), wenn es anderswo bei einer Nichteinladung (der Götter) bleibt.

7. Ferner bei einer Einladung im pitrya-Ritual und beim Tieropfer.

8. Und viele mögen in dieser Nacht Speise genießen.

9. Bei Tagesanbruch möge man das Vollöffel-Opfer darbringen, -

865 S. Anm. 693 zu II, 14, 5.

${ }^{868}$ BVWh XXIV (X, 187, 1).

${ }^{867}$ RV VIII, 11, 6; X, 141, 3; RVIKh wie in Anm. 866.

${ }^{868}$ ĶV X, 187, 1-5; RVIKh wie in Anm, 866. Dies ist die anuväkyă (Pray.).

${ }^{860} \mathrm{RV} \mathrm{X}, 9,6$. Dies ist die yajjyā (Pray.).

870 Pray.

871 RV VII, 59, 9.

878 R.V VII, $59,8$.

873 Also die dritte.

874 RV VII, 59, 10.

$875 \mathrm{RV}$ VII, 56, 14.

${ }^{870}$ So heiBen bestimmte, mit latter Stimme rezttierte yajus-Formeln; vgl, KB II, 2.5-6; V, 6; VIII, 8; XII, 1; XXVI, 5; XXVIII, 1.5; SB XI, 2, 1, 6; AA II, 3, 6; Käty I, 3, 1 .

877 Kolmm. 
10. wenn ein Stier brüllt, -

11. oder wenn es dommert.

12. Einige veranlassen den ägnüdhr $a^{878}$ zum Brüillen, (ihn) als Brahmanensohn ansprechend.

13. Wenn $\operatorname{sie}^{879}$ den hotar auffordern, (dann lauten) seine anuvälyyă und yăjyă: »Gefüllt, o Löffel, fliege hinweg80, gut gefüllt fliege wieder her ${ }^{881}$. Wie eine Ware wollen wir beide ${ }^{882}$ Trank und Nahrung tauschen ${ }^{883}$, o Hundertkräftigerl« ${ }^{884}$ (bzw.) "Gib mir, (damn) gebe ich dir $^{885}$; ïbergib mir, (dann) ïbergebe ich dir $^{886}$. Als ein Äquivalent vergilt (mir) $!^{887} \mathrm{Wer}$, ach, mag einem, der nichts (dafür) gibt, (etwas) geben $«^{888}$

14. Für die Tändelnden Maruts ist die nächste (isti).

15. "Und die Menschen sollen sprechen ${ }^{889}$ (und) "Dieser, tüchlig, unbezwingbar . . . ${ }^{800}$ (sind) versteckt auf den Vịtratöter bezogen ${ }^{891}$.

16. (Die eigentlichen anuvākyā und yäjyă sind:) »Spielend eure Marutschar ... $\ll^{892}$ (und) »Wie Rosse die Maruts gewandt $\ldots \ll^{893}$

17. »Als angenehmer Hausfreund ... «894 (und) $» O$ Agni, erstarke zu großer Herrlichkeit ${ }^{895}$ sind die beiden samyäjyăs. -

${ }^{878}$ S. Anm. 823 zu II, 16, 20.

870 Komm.: die adhvaryus.

s80 Vgl, $A V$ III, 10, 7; TS I, 8, 4, 1; ISS IX, 5; MS I, 10, 2; VS III, 19; SB II, 5, 3, 17; Ap VIII, 11, 19.

881 S. Anm, 880 außer $\mathrm{Ap}$.

B8z S. Anm, 881 .

B\$3 RV VIII, 93, 28.

B84 Beiname des Gottes Indra.

8s5 SB II, 5, 3, 19; sonst wie in Anm. 881. Dieser Sprueh ist die Generaldevise des gesamten Opfersystems I

886 S. Anm, 885.

${ }_{887} \mathrm{KS}$ IX, 5; MS I, 10, 2. apäm itham ist jedenfalls verderbt tund apämilyam zul lesen.

888 MS I, 10, 2. Statt ambã dadate ist ambadadate (aus amba + adadate) zu lesen.

${ }^{889} \mathrm{~S}$. Anm, 773 zu II, 16, 7.

890 RV VIII, 79, 1.

B9k Der holar reztiert diese Verse zu den beiden äjyabhägas (Komm.).

892 RV I, 37, 1.

893 RV VII, 56, 16.

894 S. Amm, 626 zu II, 11, 9.

895 S. Anm. 627. 
Mit Ausnahme der Molkenspende und des Reinigungsbades ist das Māhendra-Ritual (schon) beim varunapraghäsa beschrieben worden.

18. Aber an die Stelle der siebenten und der folgenden havisSpenden (tritt als Gottheit) Indra, Indra der Vỵtratöter ${ }^{896}$ oder der Große Indra ${ }^{897}$, und Viśvakarman ${ }^{898}$.

19. "Zu uns, o vịtatötender Indra ..." ${ }^{899}$ (und) »Dir wurde gegeben zu großer Indra-Macht ... ${ }^{900} «^{901}, » O$ Viśvakarman, durch die Opfergabe gewachsen . . . $\ll^{802}$ (und) »Deine höch" sten (und) deine niedrigsten Stätten ${ }^{003} «^{004}$.

Ende der 18. kaṇdikā

II, 19

1. Nachdem man aus dem Südfener Feuer herbeigeholt hat, wird ein Manenopfer ${ }^{905}$ (auf diesem ${ }^{806}$ ) bereitet.

2. Dieses hat am Ende einen Heilsspruch.

3. Die geflüsterten (Formeln) werden weggelassen; ausgenommen sind (die Formel) $»$ Den holar erwähltest du«, las dem vașal-Ruf folgende mantra und der him-Laut.

4. Die (mit dem Antlitz) nach Osten gerichteten Riten (erfolgen) bei diesem (Manenopfer mil dem Blick) nach Süden ${ }^{90 ?}$.

5. Entsprechend diesem Zusammenhango08 (sind) die anderen (Himmelsrichtungen einzurichten).

6. „Bereitwillig setzen wir dich nieder $\ll^{809}$, diesen (Vers rezi-

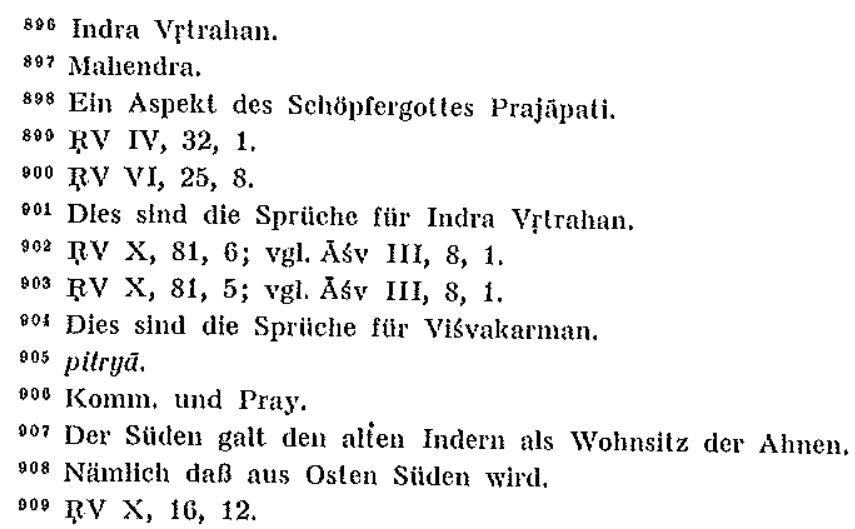


tiert der hotar) dreimal, ohne Luft zu holen. Dies sind die $»$ Entflammungsverse ${ }^{910}$.

7. Nit dem letzten $O m$ derselben (verbinde er die Formel:) "Führe die Götter (und) Ahnen dem Opferveranstalter zu! « ${ }^{911}$; dies ist das (rechte) Mittel ${ }^{912}$.

8. Statt »Den Agni zum holar'Ant führe her ${ }^{913}$, (deine) eigene Größe führe her! « ${ }^{914}$ (zu rezitieren), lasse er den Agni Kavyavāhana herbeiführen.

9. Und diesen schiebe er (auch beim letzten Voropfer vor den »Buttertrink-Versen $^{915}$ ein.

10. Im süktavăka ${ }^{910}$ (tritt ersterer) an die Stelle von "Agni (hat) durch sein hotar-Amt . . . «117

11. Hier (liegt) der Unterarm nicht (auf der Erde) ${ }^{918}$.

12. An Barhis ${ }^{919}$ (erfolgen) keine Vor- und Nachopfer. - (Ferner) kein Verzehren der ida.

13. (Und also auch) kein Abwischen.

14. Beim süktavãka (erfolgt) keine Nennung des Namens (des Opferveranstalters).

15. Angeschaut (und mit) $»$ Setz dich, hotar $1 «^{920}$ angeredet, lasse er sich nieder.

16. (Die beiden äjyabhägas ${ }^{921}$ heißen) »Leben bringend $\aleph^{922}$. Mit dem linken über (den rechten geschlagenen) Schenkel, die Brahmanenschnur tragend ${ }^{923}$ tragend, vollziehen sie die Opfergaben.

\footnotetext{
910 samidhenī; s. Anm. 51 zu II, 1, 26.

911 Das mantra erscheint in dieser Form nur hier.

913 Der Komm. erkłärt dazu, welche Verse hier zu vermeiden sind.

${ }_{013}$ Vgl. SB I, 4, 2, 17; II, 6, 1, 22; TB III, 5, 3, 2; Aś I, 3, 22; Sănkh I, 5, 5.

914 S. Anm. 913.

015 Vgl. Åsv I, 5, 24 .

916 S. Anm. 800 zu II, 16, 13.

${ }^{212}$ pratika aus agnir hotrenedam havir ajușata; vgl. MS IV, 13, 9; SB I, 9, 1, 10; SănkhSrS I, 14, 15.

$018 \mathrm{Vgl,}$ zu dieser Vorschrift Āsv I, 3, 22.

019 Die personifizierte Opferstreu.

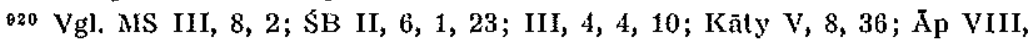
14, 22; XI, 3, 8; Măn II, 2, 1, 32 .

921. Pray.

922 Es ist jivätumantau statt jivānumantau zu lesen.

923 S. Anm. 173 zu II, 3, 21.
} 
17. Nach Süden (gewandt ist) der agnïdhra, nach Norden der adhvaryu. (Es giht) jeweils zwei anubākyās. (Er rezitiere) anderthalb ${ }^{924}$, ohne Atem zu holen.

18. „Om svadhă « $\ll^{925}$ (lautet) die Aufforderung ${ }^{926}$. „Die Manenspende soll sein $\ll^{927}$ (lautet) die Antwort ${ }^{928}$. "Anu svadhā«, »svadhā $\ll^{929}$ ist die Aullorderung ${ }^{930}$.

19. Der ăgur-Ruf (lautet:) »Ye svadhä« ${ }^{331}$ oder »Die wir svadhä rufen ${ }^{932}$. - \Svadhā namah $\ll^{933}$ ist (hier) der vașat-Ruf.

20. Die üblichen Plutierungen ${ }^{934}$ (finden auch hier statt).

21. (Die Gottheiten sind:) die Somabesitzenden Ahnen oder der von den Ahnen Begleitete Soma, die auf der Opferstreu Sitzen(len Ahnen, die vom Feuer Vexzehrten Ahnen (und) Yama.

22. "Erheben sollen sich die unteren (und) die höchsten . . «\$95 (und) »Durch dich unsere Ahnen, o Soma, einstmals . . «" ${ }^{936}$ (sind die beiden anuväkyās ${ }^{937}$ ). »Herbeigerufen sind die somaliebenden Ahnen $\iota^{938}$ (ist die $\left.y \bar{a} j y \bar{a}\right)^{939}$. - $\gg \mathrm{Du}$, o Soma, bist hervorragend an Weisheit $\ll^{940}$ (und) $\gg$ Soma (gibt) eine Milchkuh, Soma (gibt) ein schnelles Roß« ${ }^{941}$ (sind die anuvăleyās). »Du, o Soma, vereint mit den Ahnen . . . $\ll^{942}$ (ist die

${ }^{924}$ Also von der zweiten anubākyā nur die crste Hälfte.

${ }_{925}$ Vgl. SB II, 6, 1, 24; GB II, 1, 24; Mān I, 7, 6, 32; Kāty V, 9, 11; Āsv GṛS IV, 7,30 .

826 äsrävana; geht vom adhvaryu an den ăgnialhra; vgl. Āp VIII, 15, 10.

027 Vgl. Anm. 925 sowie 'TB I, 6, 9, 5; Vait IX, 11-12; $\AA_{p}$ VIII, 15, 11.

228 pratyaśrávana; vgl. $\lambda \mathrm{p}$ XXIV, 1, 10.

820 Erscheint nur hier.

930 Des adhuaryu an den hotar.

931 Erscheint nur hier'.

032 Vgl. Sảnkh III, 16, 15; $\AA_{p}$ VIII, 15, 11; Mãı I, 1, 2, 24.

${ }_{993}$ Vgl. IS XXXVI, 16; MS I, 10, 18; TB I, 6, 9, 5; SB II, 6, 1, 24; Sānkh

III, 16, 15; Āp VIII, 15, 11; Măn I, 7, 6, 34; Kāty V, 9, 12; ẢsvGṛS IV, 7, 11;

HirGṛS II, 10, 7. 11, 1. 14, 4. 15, 2.7.9.

93: Vokaldehnungen.

935 RV X, 15, 1; vgl. $\lambda s ́ \mathrm{v} \mathrm{V}, 20,6$.

936 RV IX, 96, 11.

937 S. Āsv II, 19, 17.

$938 \mathrm{RV} \mathrm{X}, 15,5$.

${ }^{939}$ Fur die Somabesitzenden Ahnen,

จง0 RV I, 91, 1; vgl. Assv III, 7, 7; IV, 3, 2.

941 RV I, 91, 20.

812 RุV VIII, 48, 13; vgl. Asv V, 19, 1 . 
$y(\bar{a} j y \bar{a})^{9: 3}$. - „O ihr auf der Opferstreu Sitzenden Ahnen, zu

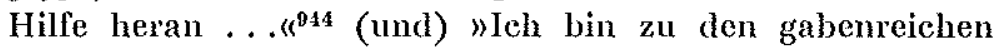
Vätern gelangt « ${ }^{945}$ (sind die anuvăkyās). »Diese Verehrung soll den Ahnen heute sein $\ll^{946}$ (ist die $\left.y \ddot{a j} y \tilde{a}\right)^{947}$. - O ihr vom Feuer Verzehrten Ahnen, kommt hierher $\star^{948}$ (und) »Die Ahnen, die hier und die nicht hier sind . . " ${ }^{949}$ (sind die anubākyās), „Die rom Feuer verbrannt und die nicht vom Feuer verbrannt sind . . . $\|^{950}$ (ist die $\left.y \bar{a} j y \bar{a}\right)^{951}$. - Die mit »Auf diese Grasstreu, o Yama, setz dich her $\ll^{962}$ beginnenden beiden (Verse sind die anwäkyă). "Den, der dahingegangen ist zu den großen Höhen . . . $\ll^{953}$ (ist die $\left.y a \tilde{j} j y \tilde{a}\right)^{954}$

23. Wenn dem Vaivasvata ${ }^{955}$ (geopfert wird), ist der mittlere $\left(\right.$ Vers) ${ }^{956}$ die $y \tilde{a} j y \bar{a}^{05 ?}$.

24. (Beim sviștakṛt ${ }^{058}$ lauten die beiden anuvākyăs:) $» D i e$ inmitten der Götter lechzend dürsteten $\ll^{959}$ (und) $» V o n$ dir, o Agni, (stammen) Erkenntnis und Gebete ${ }^{960}$; (die yajjyä:) »Er, nach alter Art durch Kraft erzeugt . . . «861

25. Agni Sviștakṛt ist (hier = Agni) Kavyavāhana.

26. Weiter ${ }^{902}$ (geht es) nach der Grundregel.

943 Fir den von den Ahmen Begleiteten Soma,

$914 \mathrm{RV} \mathrm{X}, 15,4$.

$945 \mathrm{RV} \mathrm{X}, 15,3 ;$ vgl. Aśv V, 20, 6.

Q16 KV X, 15, 2; vgl. Aśv V, 20, 6.

947 Für die auf der Opferstreu Sitzenden Ahmen.

$948 \mathrm{RV} X, 15,11$.

019 RV $X, 15,13$.

850 RV X, 15, 14.

951 Für die vom Fener Verzehrten Ahnen.

252 RV X, 14, 4; vgl, Aśv V, 20, 6.

oss RV $X, 14,1$.

05s Fir Yama.

955 Hier ein Aspekt des Yama.

950 Bei den letzten drei.

257 Also sind in diesem Falle der erste und der dritte Vers die beiden anuväkyäs.

Dies wird auch durch den Komm. bestätigh.

958 Komm.

$059 \mathrm{RV} \times, 15,9$.

960 RV IV, 11, 3.

${ }^{261}$ RQV I, 96, 1. Es ist pratnathá statt pratnayä zu lesen.

๑63 Komm.: nach dem Sviștakṛl; es wird dann nicht das linke Bein thergeschlagen usw. 
27. Oder (er führe) ferner die vașatkara $a^{903}-Z_{\text {eremonie }}^{904}$ bei den beiden äjyabhăgas (aus). (In beiden Fällen bleibt) ${ }^{985} \mathrm{der}$ mantra-Ausfall ${ }^{966}$.

28. Dann (gibt es nur) jeweils éine anuvākyā.

29. "Agni, der die Leichname fortführt . ... $\ll^{967}$ (und) $» D u, o$ Agni Wesenskenner, angefleht . . « $\ll^{908}$ (sind dann) die beiden sainyājyäs.

30. Nachdem sie beim Verzehren Atemspeise verzehrt haben ${ }^{969}$, mögen sie (die $i \grave{l}(\bar{a})$ auf die Opferstreu werfen. Wenn (alles) vollzogen ist oder vor den beiden anuyäjas verehren sie, nach Süden gewandt, das Südfeuer.970.

31. Nicht (nach Süden) gewandt, wenn kein Transport entnommenen Feuers ${ }^{371}$ (stattgefunden hat).

32. $" \mathrm{Mit}^{972}$ dieser Ausbreitung, die Taten erzengt ${ }^{973}$, ist er eine feurige, weite Bahn zur ${ }^{974}$ Ausdehnung ${ }^{975}$. Er stieg die Stütze empor bis zum Gipfel des Süßen ${ }^{976}$. Seinen eigenen Körper trieb er hin zum Selbst ${ }^{97 z^{\prime}}$.

33. ( $\mathrm{Er}$ verehre aber dorthin) gewandt die beiden anderen (Feuer).

${ }^{963}$ D. h. er vollzieht den vașal-Ruf.

oos Komm.: an Stelle des suadhä-Rufes.

${ }^{965}$ So deutet die Pray. das hier besonders schwer verständliche sülra.

060 S. II, 19,3 .

987 RV X, 16, 11. Die Deutung von kravyavahana, das RV tund Aśv haben, folgt Grassmann. Die Parallelstellen in VS und KS sowie Pray. weisen allerdings die hier plausiblere Form kavyavāhana auf.

968 RV X, 15, 12.

809 D. h. an der $i d a$ gerochen haben.

970 Fortsetzung in sülra 32.

a71 S. II, 19, 1 .

072 Fortsetzung aus sütra 30.

973 Vgl. AV VII, 3, 1; TS I, 7, 12, 2; KS IX, 6; XIV, 3; XXXIII, 4; XXXVI, 13; MS I, 10, 3; Sãnkh III, 17, 1; $\lambda_{p}$ VIII, 16, 5; XVIII, 2, 3; Mãn I, 1, 2, 15; Käty XXV, 6, 10; Sānh GṛS V, 8, 2.

974 Oder: zum Gewinn.

975 S. Anm. 973 ohne KS XXXIII; XXXVI; Ap; Măn; Sãn̉khGṛS.

976 S. Anm. 975.

277 Vgl. MS I, 10, 3; AA I, 3, 4; Sāńkh III, 17, 1. - Das ganze, der atharvanischen Mystik entstammende mantra ist offenbar absichtlich dunkel gehalten.

11 Acta Orientalla, XXXIV 
34. Den ähavanūya mit der parkti978: $" \mathrm{Dich}^{979}$, den einen schönen Anblick Gewährenden . . « $\ll^{980}$

35. Den gärhapatya (mit:) $\gg A g n i$, den ehre ich ... «881 (und zwar) mit dem Einzelvers, nicht mit der (ganzen) Hymne.

36. Dann gehen sie rund ${ }^{982} \mathrm{um}$ diesen $^{983}$, wobei sie flüstern: "Nicht wollen wir verlassen ... ${ }^{984}$ (und) $» O$ Agni, (sei) du unser .... $\ll^{985}$

37. Nachdem sie östlich (vom gärhapatya ${ }^{980}$ ) die beiden gärhapatya-Hymnen vollendet haben, wenden sie sich nach links und begeben sich zu den tryambakas ${ }^{987}$.

38. (Was) dafür die adhvaryus als Ritus (lehren, das) lernt ${ }^{988}$ man.

39. Nachdem sie zurückgekehrt sind, opfem sie für Aditi ${ }^{989}$.

40. (Hier sind die beiden äjyabhägas ${ }^{900}$ ) "gedeihenbringend «, und die beiden Zusatzverse (stehen) im viräj-Metrum.

Ende der 19. kaṇḍikā

II, 20

1. Am fünften Vollmondstag (opfert man) mit dem sunäsïrïy $a^{991}$ (-Ritual).

${ }^{\text {87 }}$ Ein Metrum aus $5 \times 8$ Silben.

079 Angeredet ist Indra.

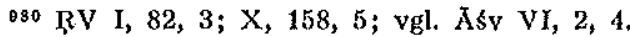

Q81 RV V, 6, 1; vgl, Aśv IV, 13, 7; VII, 8, 1; X, 10, 2.

Q83 KSomm.: mach rechts.

983 den gärhapalya.

${ }^{84}$ S. Anm. $256 \mathrm{zu}$ II, 5, 4. - Es ist mä pra gāma statt mã prama ma zu lesen.

Q85 RV V, 24, 1; vgl. Āsv VIII, 2, 3.

$O B B$ Konm.

${ }^{887}$ Dies sind drel dem Gotte Rudra gewlchte Kuchen; vgl. TS III, 2, 2, 3; KS XXXVI, 14; VS XXIV, 18; VSK XXVI, 4, 3 ; KB V, 7 ; JB I, 40; TB I, $4,10,9.6,8,1.10,5$; Ś II, 6, 2, 1.9.11-12.14; SBK I, 6, 2, 1.5-7; Ăp VIIl, 17-19.

888 Komm. : tad asmadiyair api kartayyam das ist auch von den Unsrigen (dem hotar etc. und dem Opferveranstalter) zu machen.

989 Komm, ausdrüicklich: adilir atra devatä nädilyah.

900 Pray.

-91 Wörtlich: auf Schar und Pflug bezogen, Name des vierten parvan; für ein Tertialopfer naturllch ein Widerspruch in sich; das $s$. ist daher als spätere Zutat 
2. Oder vor jenem Ereignis.

3. Mit Ausnahme der Molkenspende (-riten sind die hier vorgeschriebenen Riten mit denen) vom vaiśvadeva (-parvan) identisch. Aber an die Stelle der sechsten und der folgenden Opferspenden (treten) der mit einem Vielgespann Versehene Vāyu oder Vāyu, Schar und Pflug ${ }^{992}$ oder Indra mit Schar und Pllug oder Indra mit Schar, Sūrya als letzter.

4. „O Vāyu, Trinker des klaren (Saftes), wende dich uns zu $1^{903}$ Mit denen du dich zum Frommen begibst . . .994 - Du uns, o Gott, im Geiste . . .955 Wer dem Herrn, (ver) dir Opfergabe bringt ... ${ }^{896}$. - O Schar und Pflug, erfreut euch an dieser Stimme ${ }^{097}$ Zum Gedeihen sollen unsere Pflugscharen das Land umpflügen ${ }^{098}$. - $O$ Indra mit Schar und Pflug, wir rufen (dich) auf diese meine Seite ${ }^{999}$. Er möge uns in den Kämpfen voranhelfen ${ }^{1000}$. Begierig nach Pferden, begierig nach Rindern, begierig nach Beute ... 1001. - Wir wollen rufen den gabenreichen Indra mit der Schar ${ }^{1002}$. Begierig nach Pferden, begierig nach Rindern, begierig nach Beute $\ldots{ }^{1003}$. - Rasch vorwärtsdringend, überall sichtbar . . 1004

zum Opferritual zu betrachten und gitt als symbolische Aneignung des 13. (Schalt-) Monats. Vgl. auch MS IV, 3, 3; VS XXIV, 19; VSK XXVI, 4, 4; KB V, 8; VI, 15; PB XXV, 4, 1; TB I, 4, 10, 2-3.9; SB II, 6, 3, 2.5.10-11.13; V, 2, 4, 4; XI, $5,2,6.9$; ŚBK I, 6, 3, 2.5; GB II, 1, 26; Ӑp VIII, 20-21.

082 Im Anschluß an die Aufassung R. v. Roths.

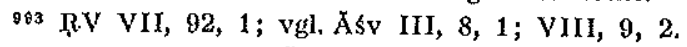

994 RV VII, 92, 3; Xśv wie soeben. - Dle beiden Verse sind anuväkya und yajyä für den mit chinem Vlelgespann Versehenen Vãyu.

005 RV VIII, 26, 25.

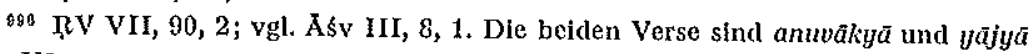
für Vāyu.

297 RV IV, 57, 5.

898 RV IV, 57, 8. Die belden Verse sind anuvakya und yajyã für Sunäsirat.

800 Erscheint nur hier.

1000 RV I, 81, 1. Dlese Verse sind antuākyä für Indra mit Schar und Pfug. $1001 \mathrm{RV} X, 160,5$. Dies ist de entsprechende yajyā.

1002 RV III, $30,22.31,22.32,17.34,11.35,11,36,11.38,10.39,9.43,8$. $48,5.40,5,50,5 ; \mathrm{x}, 89,18,104,11$.

1003 RV X, 160, 5. Die beiden Verse sind antwākya und yajyă für Indra mit der Schar,

1004 RV I, 50, 4; vgl, Asv IX, 8, 3. 
Das strahlende Antlitz der Götter ist aufgegangen ${ }^{1005 . 《 ~ S o ~}$ (lauten) die yājyās und anuvăkyās.

5. Nachdem (dies) vollendet ist, bringe man ein Somaopfer dar; wenn man es nicht kann, ein 'Tieropfer.

6. Oder ement die 'Tertialopfer, (ja) oder erneut die Tertialopfer.

Ende der 20. kaṇdikã

\section{ENDE DES II. ADHYÄYA}

1005 RV I, 115, 1; vgl. Xśv III, 8, 1; IX, 8, 3. Diese beiden Verse sind anuvakyă und yajyã fúr Sưrya. - Hiermit schließt die Behandlung der cālarmāsyāni und damit des gesamten havis-Rituals ab. 


\title{
A GRITIGAL STUDY OF THE TAMIL BRAHMI INSGRIPTIONS
}

BY

\author{
R. PANNEERSELVAM
}

\section{Brahmi inscriptions:}

\section{Introduction}

Inscriptions found written in Brahmi script are labelled as Brahmi inscriptions. Beginning from the period of Asoka and in successive periods inscriptions in Prakuit language were written in Brahmi seript in northern India, and the inscriptions in Tamil language written in this script were found in the natural caves of Tamilnad. The great distance between these two geographical divisions-Tamilnad in the south and the Asokan empire in the north-and the addition of new letters in each division to satisfy linguistic requirements forced scholars to consider two different varieties of script, the northern and the southern. Palaeography of these inscriptions has been done in Indian Epigraphy and South Indian scripts. ${ }^{1}$

The major distinction between the two varieties is that the northern has additional letters for voiced and aspirated sounds, and the script is more angular. On the other hand, the southern has no signs for voiced and aspirated sounds with the exception of $d h$ and $s$, but there are extra letters for the sounds $11 \mathrm{n}$ ! and $i$ which are not common in the northern variety ${ }^{2}$ and the script as a whole is more cursive in character. Inscriptions in southern variety are taken up here for critical study.

Inscriptions found in Tamilnad are comparatively short ranging from one line of six letters (no. 20), to four lines of 61

1 C. Sivaramamurtiy.

* K. V. Subramaniya Aiyar. 
letters (no. 38). These inscriptions date to the period between the second century B.C. and the fifth century A.D. ${ }^{3}$ These dates have been determined on the basis of palaeography. The historical evidences and the socio-cultural relations known from the contemporary inscriptions found elsewhere also help to confirm this dating.

\section{Prakrit and Tamil:}

The languages underlying the Brahmi script in these inscriptions are Prakrit and Tamil, and there is evidence of translation from Prakrit into Tamil. For instance most of the legends relating to Jain and Buddhist monks are considered to be translations from Prakrit. This problem has been discussed in my article 'Further light on the bilingual coin of Satavahanas'. 'The legends of the coin run as follows:

Prakrit: vasitiputasa siri satakaṇisa raaño

Tamil: vacittimakanku tiru catakaṇiku aracanku

(the coin of Vasitiputa siri Satakani king). If we compare both legends, we shall find that the Tamil legend is a word by word translation of the Prakrit legend. In it the genitive case endings -sa and -o of Prakrit legend are translated as -ku in Tamil. The proper names are merely transcribed in a Tamil form. A detailed analysis of all the available Brahmi inseriptions both in Prakrit and Tamil will give further good results on this problem.

\section{Decipherment and earlier studies:}

The history of the decipherment of the Tamil Brahmi inseriptions has been discussed by the late cminent scholar and epigraphist K. V. Subramaniya Aiyar in The earliest monuments of the Pandya country and their inscriptions, ${ }^{5}$ who first published the readings of these inseriptions, and Kamil Zvelebil in the introduction to his article The Brahmi hybrid Tamil inscriptions, ${ }^{6}$

\footnotetext{
8. Mahadevan.

4 R. Panneerselvam.

- KK. V. Subramaniya Aiyar.

- Isamil Zvelebil.
} 
in which he has made a formal study. The details of the decipherment of these inscriptions and their publications are given by I. Mahadevan, in his Corpus of the Tamil Brahmi inscriptions.? And therefore it is needless to repeat them here.

As far as the linguistic study of these inscriptions is concerned the works of T. P. Meenakshisundaram ${ }^{8}$ and Kamil Zvelebil ${ }^{9}$ should be mentioned here. But their analyses are based on earlier readings, chiefly those of $K$. V. Subramaniya Airar, which now have been considerably changed in the Corpus of 1968. The present decipherment offers some new readings which if accepted, will necessitate more formal analysis.

\section{Corpus for the present critical sludy:}

The material taken here for the critical study is from the 'Corpus of the Tamil Brahmi inscriptions' published as one of the articles in Seminar on Inscriptions. ${ }^{10}$ So far 76 inscriptions have been deciphered by I. Mahadevan, now the managing director of Modern Bakeries India, New Delhi, who has taken much pain to take his own estampages of these inscriptions.

Of these 76 inscriptions 46 were published with facsimiles. These facsimiles constitute the basis of our corpus for critical study. They are given with my own serial numbers and a table is provided for the reader wishing to refer to the Corpus at the end of the paper.

\section{Emendations:}

These facsimiles were read by me independently of other existing readings. Even if the reading of Mahadevan is substantially correct, I have made a few emendations here and there and some word divisions which have consequent effects on the translation.

For instance, in the first inscription I have divided the phrase 'nanta-a siriykuvan' as 'nanta-a siriy kuvan' considering 'kuvañ' as a personal name (cf. 2 and 3 ), while Mahadevan has taken

\footnotetext{
? I. Mahadevan.

8 T. P. Meenakshisundaram.

- Kamil Zvelebll.

10 I. Mahadevan.
} 
the phrase as 'nanta asiriyka uvan' and translated it as 'teacher' (living) 'yonder'.

In the Kilavalavu inscription the eighth letter stands for 'tu' and not for 'ti' and I have suggested 'tonțu' (duty/service) and the following word as 'ulavan' (possessor) both to mean devotee.

The inscriptions 33 and 34 of his Corpus are taken as one single inscription (25). In inscription 35 the 'iila' is a caste name (tree climbers), while Mahadevan has taken it as a place name, Ceylon. But in personal discussions Mahadevan agreed that the suggestion of 'ilia' as a caste name was possible.

Another notable difference in my reading is in the Pukalur inseription. 'There I read 'kurummakal' (and not 'kurummakkal' $=$ sons) and translate it as granddaughter because the granddaughter is called 'kiiran noori' (Noori the daughter of Kiiran). Kiiran, who is mentioned in the preceding epigraph as 'Kiiran korra', is described as the daughter of Pitantai. These conjunctures apparently lead us to translate the phrase 'Pifantai kurummakal' as granddaughter of Piţantai, and Noori as a female personage. I divided the compound as 'kiiran noori' in 41 and 'vaanilkan natti' in 42 , differing from 'kiiran oori' and 'vaanikan atti'.

The first word of inscription 43 is only partially extant, and we guess it to be [ven]naakan, as found in the early Tamil works. ${ }^{11}$

11 Early Tamil works generally are called Canka ilakkiyam, consisting of elght anthologies and ten idylls: Narrinai, Kurunlokai, Ainkurumuru, Patirruppattu, Parfpaafal, Kalittokai, Akanaanuuru, and Puranaanuuru: and Tirumurukaarruppatai, Porunaraarruppaţal, Cirupaanaarruppaţai, Perumpaanaarruppatai, Mullaippaațț, Neţunalvaațai, Kurincippaațįu, Maturaikkaańcl, Pạținappaalai, and Malaipațukațaam.

Tańkaal porkollan veṇnaakanaar (Veṇnakanaar, the goklsmith of Tankaal) Akam-48, 108, 355: IKuruntokal - 217: Narrinai - 313.

Maturai kollan veṇiakanaar (Veṇnakanaar, the smith of Maturai) Akam-363. Ilańkiiran - Akam - 3, 225, 239, 289, 299, 361, 371, 395, 399: Kuruntokat - 116: Narrinai $-\overrightarrow{3}, 62,113,269,308,346$.

İankitrantai (father of Ilanktiran), Kurontokai - 148.

Ifankilirantal may be identified with [ven]paakan of the inscription (no. 43). In that inseription Ilankiliran was described as the son of [ven]naakan ([ven] naakan makan lankilran): he is a goldsmith and a native of Maturai according to the literature: and he is residing in Karur since his name is found in the inscription found in Pukalur. 
The reading of the Araccalur epigraph is very difficult, and we make here some tentative suggestions. R. Nagaswamy, the Director of the Department of Archacology, Madras, has read the beginning of the lines as 'eluttu puparuttaan' (and interpreted it as, he who has composed the letter's/rhythm) and has discussed it elaborately in the Tamil magazine 'Kalaimakal'. ${ }^{12}$ But his reading involves some assumptions about the text that do not seem to be proved. As far as the first line of the inscription is concerned the reading of Mahadevan (elu taanam panvittaan = seven beds (charities) caused to be made) is not satisfactory either, and I suggest my reading as 'elutu nampan vattan' and interpret it as 'the writing of Nampan vattan'13 with some hesitation.

\section{Historical importance of these inscriplions:}

Of all the Brahmi inscriptions the Pukahr epigraph is the most interesting. In it, we find a complete genealogy of the kings who ruled from Karur, an ancient capital of the Chera kings, who ruled under the family name 'Irumporai'. This particular inscription was elaborately discussed by me, with reference to the literary sources in 'An important Brahmi Tamil inscription: a reconstruction of the genealogy of the Chera kings'.14

The inscriptions 58,59 , and 65 as given in the Corpus, speak of Pitantai and 'Kiiran korra', and remind us of a 'vaanavan maravan pițan' and 'Pittan korran' (a chief under the Chera kings) mentioned in the early Tamil works. (Akam-143: Puram168,170 ).

The Mankulam inseription (no. 1) speaks of a Pandya king Nef̧uncaliyan and his servant called 'kafalan valuttiy'. This name Kațalan is also found in the early Tamil works (Akam-81) as 'maa van katalañ' (the charitable kaţalan) as an epithet of the Pandyan king Neţuñceliyañ.

12 R. Nagaswamy.

13 In an inscription of 8 th century A.D. we flnd the name 'Nampan antai' (I.P.S. No. 241): In the Velurpalayam grant an offcer who is executing the royal order had the name 'Nampan': it belongs to the 9 th century A.D. (S.I.I. vol, 2-5, No. 98).

is $\mathrm{R}$. Panneerselyam. 
Identification of place names in early Tamil works:

The place names found in the inscriptions to some extent help to identify or to confirm certain places mentioned in the early 'Tamil works. The most important are Nalluur (Pattuppaatfu3), Kumuliñ̃aalal (Akam-160), and Teemutukuñ ram (Akam-197).

Nalluur is described as located in Itaikalinaatu and the place of the poet Nattattan. This can be equated with Nalliyuur found in the inscription of Pukalur near Karur. Hence this place may be found somewhere around Karur. The place names 'Nallipaalayam' and 'Nalliyampaalayam' of recent times which are found distributed around this region also help us to fix Nalluur in Karur.

Kumul-turr, which is found in the inseription 22, is another place which can be identified with Kumuliñaalal, a place from where the poet Nappacalai hails. This Kumulinaalal may be located in the Pudukkoltai division of Tiruchirapalli district since the epigraph in which the name Kumul-uur occurs is found in Cittamnavacal, a place in Pudukkottai.

The name Teenuur is found in the Mamantur epigraph. Mamantur is in the Chingleput district and very near to the Telugu region. This place may be identified with Teemutukungam of the Tamil works. Teemutukun ñam belonged to a certain chief Kanpun elini. The normal tendency of the poets describing the sufferings of the heroine on parting from the hero, is that they used to say even if he crossed over the country of the Vadugas (the Telugus), he would not stay longer and he would come back soon. Hence, Teenutukungram so described will be considered as a place in the border region of Tamilnad, and that the epigraph found in such a region is a confirmation of the identification.

\section{Linguistic peculiarities:}

These inscriptions, apart from historical and sociological interest, have some linguistic importance. The change of $s$ into $y$ and $c$ in Tamil is worthy of mention here. In the Mankulam inscription (no. 1), this $s$ is still written as $s$; it belongs to 2-1 century B.C. In the Pukalur inscription (no. 38) we find it written as $y$ in the word 'cenkaayapan'. On the coin of Sata- 
vahanas referred above, ${ }^{15}$ we find $c$ in the place of $s$ in the word 'vacit!i'.

The dot over the primary consonants which is a characteristic feature of the Tamil script, is not found in these inscriptions, while it is found on the coin mentioned above. The use of a dot to show the short variety of $e$ is found in the Araccalur inscription, which is dated to a later period. The rules are to be found in the earliest Tamil grammar, Tolkaappiyam, which dates back to the pre-Christian era, 'meyyin iyarkai pulliyolu nilaiiyal' (the nature of the consonants is to have the dot), and 'ekara okarattu iyarkaiyum argec' (the nature of the letters ' $e$ ' and ' $o$ ' is the same).

Distribution of the inscriptions:

These inscriptions are distributed geographically as follows:

$\begin{array}{lclr}\text { Araccalur } & -1 & \text { Tiruvatavur } & -2 \\ \text { Alakarmalai } & -10 & \text { Pillaiyarpatti } & -1 \\ \text { Anaimalai } & -1 & \text { Pukalur } & -6 \\ \text { Karunkalakuti } & -1 & \text { Mankulam } & -5 \\ \text { Kilavalavu } & -1 & \text { Mamantur } & -1 \\ \text { Konkarpuliyankulam } & -3 & \text { Muttuppatti } & -1 \\ \text { Cittannavacal } & -1 & \text { Mettuppatti } & -8 \\ \text { Tirupparankunram } & -2 & \text { Vikkiramankalam } & -2\end{array}$

\section{Summary:}

This critical study consists of four parts.

1. Lexicon, in which all the words are given with their English equiralents and grammatical notes, followed by the number of the inscription in which the particular word occurs. The words are segmented to the smallest possible unit, and also given in compounds as they occur in the inscription.

2. Classification of names and culture-bearing words. This will help the reader to compare names in the early Tamil works, as well as give information about social and cultural affairs of that period.

\footnotetext{
1s R. Pannecrselvam.
} 
3. Grammar, in which is given a sketch of the main functional elements.

4. Text, consisting of transliteration, translation, and the facsimiles of the Tamil Brahmi inscriptions.

\section{Acknouledgement:}

I acknowledge with thanks the kind permission of the Director of the Department of Archaeology, Madras, and I. Mahadevan, friend and promoter of my research in this study of Brahmi inscriptions to reproduce the facsimiles. The facsimiles were arranged so as to come out clearly, and slightly differently from the order found in the 'Corpus'. I also thank Eric Grinstead, of the Scandinavian Institute of Asian Studies, for his fruitful suggestions in making this paper more readable.

\section{System of transliteration:}

[Except in the place names given in the introduction and elsewhere.]

Modern Tamil script is given first and next is the Brahmi script available in the facsimiles: scripts for [ai] and [au] are not available in them; and there is no distinctive script found for [o] and [oo].

There are two non-Tamil scripts listed at the end of the table.

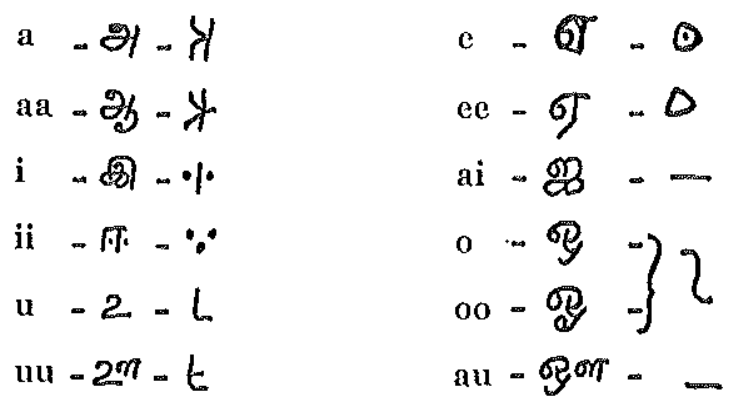


A CRITICAL STUDY OF THE TAML BRAIMI INSCRIPTIONS

$$
\begin{aligned}
& \text { k }-\infty-t \\
& \mathrm{y}-w-w
\end{aligned}
$$

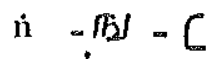

$$
\begin{aligned}
& r-g-1 \\
& \text { c }-d-d \\
& 1-\sigma-V \\
& \text { n }-\sigma-n \\
& v-a-b \\
& !-1-C \\
& 1-5-9 \\
& \text { 1) }-0007-I \\
& 1 \text { - orp - V } \\
& t-\$-k \\
& n-b-1 \\
& r-g-\delta \\
& p-u-l \\
& n-\sigma r-c \\
& m-10-t \\
& s-o n-\alpha \\
& \mathrm{dh}-\omega=D
\end{aligned}
$$

\section{List of abbreviations:}

Adj. B. - Adjective Base

Akan. - Akanaanuuru

Ap. - Appositional

Cau. - Causative

C.N. - Conjugated Noun

'Corpus' - Corpus of the Tamil Brahmi Inscriptions

D.N. - Derived Noun

F.V. - Finite Verb

I.A.T.R. - International Association of Tamil Research

I.I.J. - Indo Iranian Journal

Inf. - Infinilive

I.P.S. - Inscriptions of Pudukkoltai State

N. - Noun

Pn. - Pronoun

Pnag. - Person, Number, and Gender

Puran - Puranaanuuru

R.P. - Relative Participle

S.I.I. - South Indian Inscriptions

St. - Stem

T.M. - Tense Marker

V.N. - Verbal Noun 


$$
\begin{array}{ll}
\varnothing & - \text { Zcro } \\
- & - \text { Optional } \\
+ & - \text { Obligatory }
\end{array}
$$

\begin{tabular}{|c|c|}
\hline a & - excrement, 31 \\
\hline altuvaayi & - personal name, N. 37 \\
\hline atitfaanam & - abode, N. 42 \\
\hline atiţaañam & - abode, N. 22 \\
\hline atai & - sage/father, N. 20 \\
\hline antuvan & - personal name, N. 34 \\
\hline antai & - sage/father, N. $3,14,15,16,17,20$ \\
\hline antaiy & - sage/father, N. 12 \\
\hline $\operatorname{aman} .$. & - amaṇnañ N. 39 \\
\hline amanuman & - Jain monk, N. 38, 39 \\
\hline aralta & - personal name, N. 37 \\
\hline ari-itan & - personal name, N. 6 \\
\hline $\operatorname{aritan}$ & - personal name, N. 37 \\
\hline aritin & - personal name, N. 23 \\
\hline ariyli & $\rightarrow$ personal name, N. 14 \\
\hline ava-um & - they and, Pn. 32 \\
\hline arutta & - which (was caused to be) cut, R.P. 38 \\
\hline arupita & - which was caused to be cut, R.P. 39 \\
\hline aruvai & - eloth, V.N. 33 \\
\hline asutan & - personal name, N. 3 \\
\hline aaka & - while becoming, Inf. 38,39 \\
\hline aatan & - personal name, N. 38,39 \\
\hline aatan-een & - Aatan I, Ap. 9 \\
\hline aatana-a & - Aatan's, N. 24 \\
\hline aatanceellirumpurai & - personal name, N. 39 \\
\hline aatanceellirumporai & - personal name, N. 38 \\
\hline aay & - a dynasty, N. 35 \\
\hline itta & - this, Pn, 10 \\
\hline itta-a & - this, Pn. 1 \\
\hline ita & - this, Pn. 2 \\
\hline iravaatan & - personal name, N. 15 \\
\hline
\end{tabular}

\section{Lexicon}

* hyphen stands for hiatus or to show the pre-sandhi forms. 


\begin{tabular}{|c|c|}
\hline irumpurai & - a dynasty, N. 39 \\
\hline irumporai & - a dynasty, N. 38 \\
\hline iḷańkał̣unkoo & - personal name, N. 38,39 \\
\hline ilankiiran & - personal name, N. 43 \\
\hline ilankoo & - prince, N. 38,39 \\
\hline iḷancaṭikan & - personal name, N. 2 \\
\hline ilam & - young, Adj. B. $2,38,38,39,39,43$ \\
\hline ilay & - young, Adj. B. 22 \\
\hline iḷayar & - servants, N. 22 \\
\hline iiva & - these, Pn. 37 \\
\hline iila & - a caste (tree climbers), N. 35 \\
\hline ulavan & - he who exists, C.N. 11 \\
\hline uraiy & - abode, N. 38 \\
\hline uraiyul & - residence, N. 37 \\
\hline utparuvan & - personal name, N. 8 \\
\hline uupaacan & - devotee, N. 7 \\
\hline uupaaca-an & - devotee, N. 8, 11 \\
\hline иuрu & - salt, N. 25 \\
\hline uur & - village $N, 13$ \\
\hline unarai & - abode, N. 7 \\
\hline $\mathrm{em}$ & - our, Pn. 13 \\
\hline eri & - personal name, N. 37 \\
\hline erukaat!uuru & - place name, N. 46 \\
\hline erukaațur & - place name, N. 35 \\
\hline elutu & - writing, V.N. 45 \\
\hline ela & - young, Adj. B. 28 \\
\hline ela-a & - young, Adj. B. 33 \\
\hline eḷ-a afan & - personal name, N. 33 \\
\hline ela cantan & - personal name, N. 28 \\
\hline ce-umi & - place name, N. 22 \\
\hline eelai-uur & - place name, N. 23 \\
\hline kațalañ & - personal name, N. 1 \\
\hline katunkoon & - personal name, N. 39 \\
\hline kanatikan & - personal name, N. 25 \\
\hline kaṇi-i & - Jain teacher, N. 3 \\
\hline kaniy & - Jain teacher, N. 1 \\
\hline kaṇimaan & - personal name, N. 44 \\
\hline karu-uur & - place name, N. 42 \\
\hline
\end{tabular}


kal

kalumaaran

kaayipan

kaaviti-iy

kaaviy

kaavuți-i

kaalitika

kaasapan

kiiran

kiiran korra

kiiran noori

ku-an

ku-ittavan

kuţupitoon

kuṭumpikan

kumul-uur

kuva

kuvan-ke

kuvira

kura

kurum

kurummakal

kuñratuu

kunru

ko!tuupitta-a

koṭuupitoon

koţala

koti-oor

koțu

kolupitavan

koțupitoon

koțuupitoon

kolavan

kolı

korra

koo

koon

cațikan
- stonc/rock, B. 38,39

- personal name, N. 29

- personal name, N. 37

- merchant chief, N. 3

- abode, N. 36

- merchant chief, N. 22

- to Kaaliti, N. 3

- personal name, N. 31

- personal name, N. $40,41,43,46$

- personal name, N. 40

- personal name, N. 41

- personal name, N. 2

- he who thatched, C.N. 9

- he who caused to give, C.N. 32

- husband, man, D.N. 35

- place name, N. 22

- personal namc, N. 3

- to Kuvan, N. 1

- personal name, N. 21

- roof, N. 8, 9

- young, Adj. 5.41

- granddaughter, N. 41

- place name, N. 37

- hill, N. 44

- which was caused to be given, R.P. 1

- he who caused to be given, C.N. 6, 7

- mat/roof, N. 9

- they who gave, C.N. 5

- (that which was) given, R.P. 11

- he who caused to give, C.N. 8, 30, 34

- he who caused to give, C.N. 3

- he who caused to give, C.N. 4

- family-he/smith, N. 24

- ploughshare, N. 28

- personal name, N. 40

- king, N. 38, 39

- king, N. 44, 46

- personal name, N. 2 


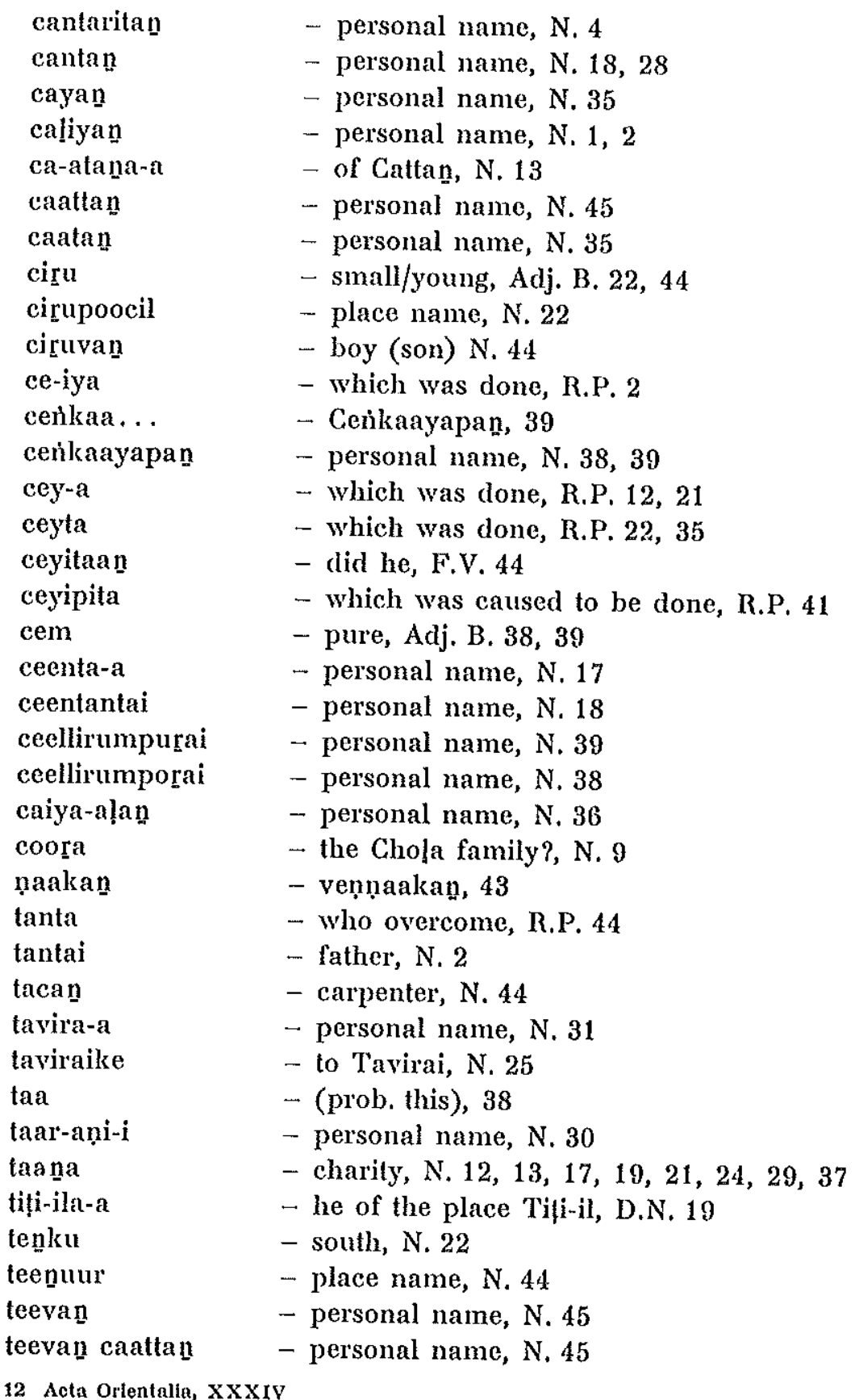




\begin{tabular}{|c|c|}
\hline toụtu & - duty/service, N. 11 \\
\hline toṇlulavan & - he who has duty (devotec), N. 11 \\
\hline natti & - personal name, N. 42 \\
\hline nata & - spiritual preceptor, N. 3 \\
\hline atan & - lord/chief, N. 37 \\
\hline nanta & - spiritual preceptor, N. 2 \\
\hline hanta-a & - spiritual preceptor, N. 1 \\
\hline ampan & $-\operatorname{good} \operatorname{man} /$ friend, N. 45 \\
\hline nalli-uura-a & - of Nalli-uur he, D.N. \\
\hline nalliyi-uur-an & - of Nalli-uur he, D.N. 40 \\
\hline laaţu & - country, N. 22 \\
\hline nikamatuu & - of merchantile guild, N. 3 \\
\hline nikamatoor & - of merchantile guild they, D.N. 5 \\
\hline neṭim & - great/elder, Adj. B. 2 \\
\hline nęincaliyan & - personal name, N. 2 \\
\hline nețu & - great/elder, Adj. B. 27, 35 \\
\hline nețucaatan & - personal name, N. 35 \\
\hline nețuñealıyan ñ & - personal name, N. 1 \\
\hline neţum & - great/elder, Adj. B. 1 \\
\hline nețumalaan & - personal name, N. 27 \\
\hline noori & - personal name, N. 41 \\
\hline paṇa-an & - servant, N. 1 \\
\hline patina...r & - unclear, 20 \\
\hline para-acuu & - personal name, N.7 \\
\hline pali & - abode, N. 23, 41 \\
\hline pali-i & - abode, N. 11 \\
\hline pali-iy & - abode, N. 1 \\
\hline paliy & - abode, N. 2 \\
\hline paakañ-uur & - place name, N. 10 \\
\hline paańlkaața & - place name, N. 6 \\
\hline paanita & - candy, N. 27 \\
\hline paata & - food, N. 37 \\
\hline paatanatan & - personal name, N. 37 \\
\hline pikan & - personal name, N. 12 \\
\hline piţantai & - personal name, N. 40 \\
\hline pițan & - personal name, N. 10,41 \\
\hline nava & - personal name, N. 3 \\
\hline iranta & - who was born, R.P. 22 \\
\hline
\end{tabular}




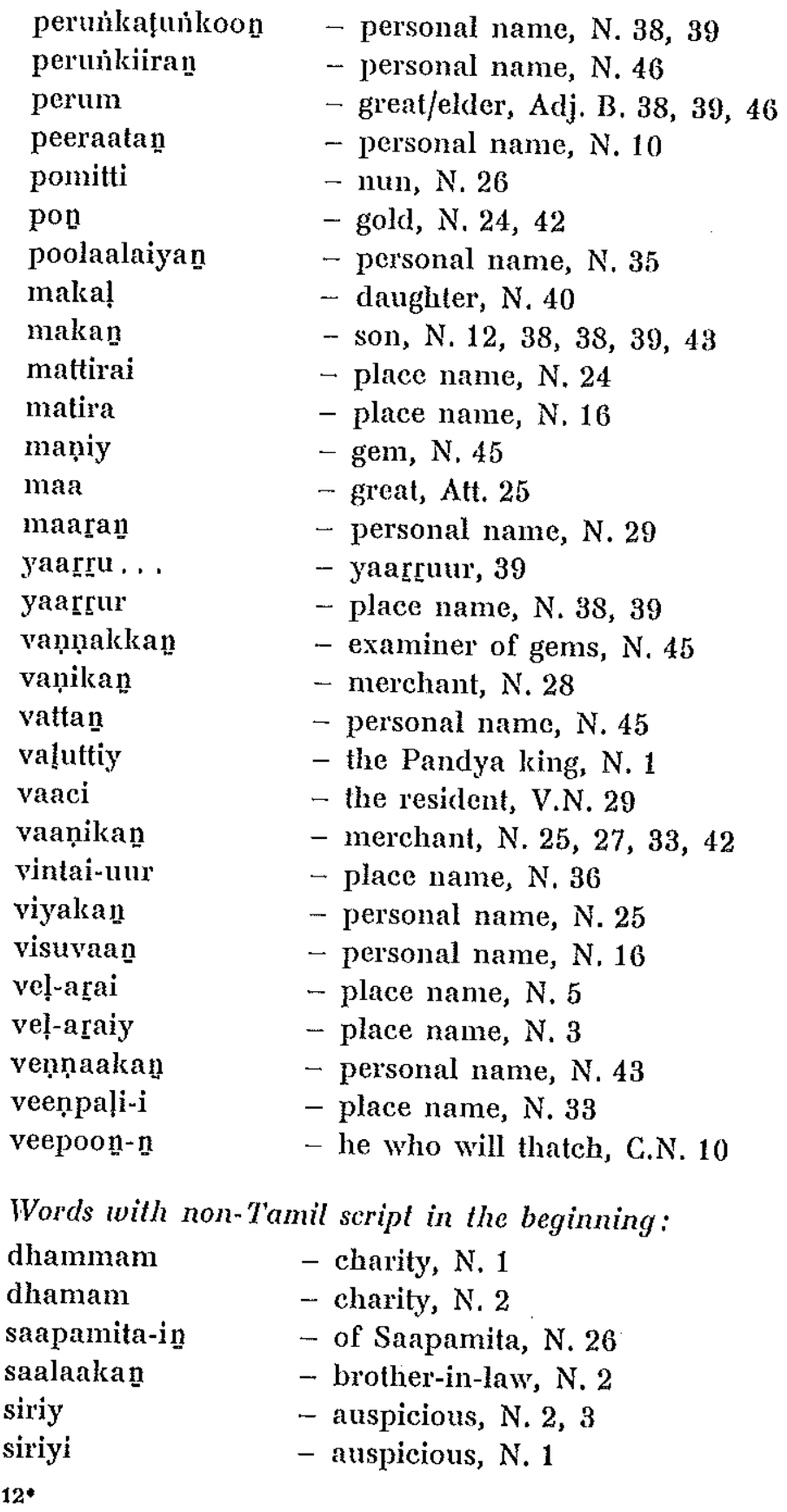


1. Place names:

\section{Classifications of names}

a. suffix: $-u r /$ aur $u, u u r=$ village erukaatur, erukaaţ̧uuru, eelai-uur, karu-uur, kumul-uur, kunnatuu, teenuur, nalli-uur, nalli-yi-uur, paakañ-uur, yaarruur, vintai-uur, veẹppali-i

b. suffix: $-i l=$ house, locative form cirupoocil, titi-il

c. suffic: -arai $=$ room, plot vel-arai, vel-araiy

d. suffix: -pali $(-p a l l i)=$ monastery, temple of Jains/Buddhists veeupali-i

e. suffix: -kaata $(k a a t u)=$ forest/dry land paankaaţa

f. common form:

mattirai, matira (Maturai) ee-umi

2. Personal names:

a. isolated forms:

attuvaayi, antuvan, aratfa, aritan, ari-itan, ariyti, asutan, aatan, uuparuvan, eri, kał̧alan, kaayipan, kaasapañ, kaaliti, kuvan, ku-an, kuva, kuvira, ca-atan, caattan, caatan, caf̧ikan, cantan, cantaritan, cayan, ceenta, caiya-alan, tavira, tavirai, taar-ani, natti, nanta, nanta-a, para-acuu, pikan, pițan, pinava, vattan, viyakan, visuvaan, saapamita

b. compound forms:

b 1. Adj. + Noun

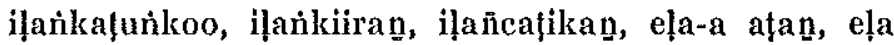
cantañ, kaţuńkoo, ceńkaayapan, nețiñcaliyañ,

nețucaatañ, nețuñcaliyan, nețumalaan,

perunkafuńkoon, peruńkiiran

b 2. Noun + Noun

kiiran korrra, kiiran nooți, teevan caattan 
b 3. Noun + antai

ceentantai (ceentan + antai)

pițantai (pitan + antai)

b 4 . Noun + alatan

iravaatan (ira + aatann)

peeraatan (peer + aatan)

b 5. other compounds

aatanceellirumporai, aatannceellirumpurai, kanatikann, kalumaaran, cantaritan, paatanatan

3. Groups of related people:

a. cał̧ikañ, ilañcațikan, nełiñcalìan

b. aatancellirumporai, peruñkaţunkooñ, iḷankałuńkoo

c. pilantai, kiiran korra, kiiran noori

d. . naakañ, ilañkiiran

4. Kinship terms:

tanlai = father; makan $=$ son; ciruvan $=$ son; makal = daughter;

kurummakal $=$ granddaughter; saalaakan $=$ brother-in-law

5. Names of dynasties:

aay, irumporai, cecl (ceeral), coora, valuttiy, callyañ, maaran

6. Royal tilles:

koo = king, koon = king, kaavuti $/$ kaavuḷ $=$ merchant chief

7. Professional names:

aruvai vaanikan - cloth merchant

uupu vaanikan - salt merchant

kolu vaanikan - ploughshare seller

paanita vaanikan - candy seller

pon kolavan $\quad-$ goldsmith

mani vanuakkan - examiner of gems

tacan - carpenter

ilayar - attendant

pana-an - servant 
8. Relgious terms:

$\begin{array}{ll}\text { amaụan } & - \text { Jain } \\ \text { upaacan } & - \text { devotee } \\ \text { tavirai } & - \text { lady disciple/devotee } \\ \text { antai } & - \text { great man/sage }\end{array}$

9. Things:

aruvai - cloth; uupu - salt; koțala - mat; kolu - ploughshare;

paanita - candy; pon - gold; maṇi - gem

10. Adjectives:

ilam, kuru, ciru $\quad \Rightarrow$ younger, small

kaţum, nețum, peru = clder, big

4. Grammar

1. Verb and Conjugation:

\begin{tabular}{|c|c|}
\hline $\begin{array}{l}\text { aru } \\
\text { arutta } \\
\text { arupita } \\
\text { aruvai }\end{array}$ & $\begin{array}{l}\text { - to cut } \\
\text { - which was cut } \\
\text { - which was caused to be cut } \\
\text { - cloth (that which was cut) }\end{array}$ \\
\hline aa & $\begin{array}{l}\text { - to become } \\
\text { - while becoming }\end{array}$ \\
\hline $\begin{array}{l}\text { urai } \\
\text { uraiy } \\
\text { unaiyul }\end{array}$ & $\begin{array}{l}\text { - to live } \\
\text { - abode } \\
\text { - residence }\end{array}$ \\
\hline $\begin{array}{l}\text { elutu } \\
\text { elutu }\end{array}$ & $\begin{array}{l}\text { - to write } \\
\text { - writing }\end{array}$ \\
\hline $\begin{array}{l}\text { kaṇ } \\
\text { kani }\end{array}$ & $\begin{array}{l}\text { - to think } \\
\text { - sage/monk, astrologer }\end{array}$ \\
\hline $\begin{array}{l}\text { kuțu } \\
\text { kuţupitooy }\end{array}$ & $\begin{array}{l}\text { - to give } \\
\text { - he who caused to give }\end{array}$ \\
\hline $\begin{array}{l}\text { ku(y) } \\
\quad \text { ku-ittavan }\end{array}$ & $\begin{array}{l}\text { - to thatch } \\
- \text { he who thatched }\end{array}$ \\
\hline $\begin{array}{l}\text { koțu } \\
\text { kotfuupitta-a }\end{array}$ & $\begin{array}{l}\text { - to give } \\
\text { - which was caused to be give }\end{array}$ \\
\hline
\end{tabular}




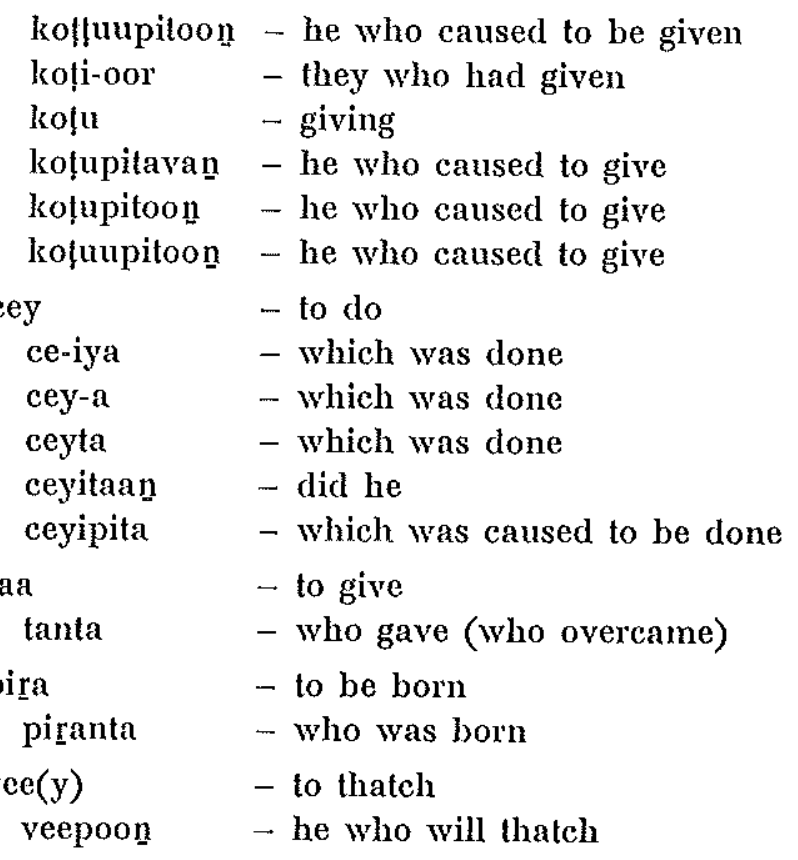

2. Grammatical elements (verbs):

1. Causative: -pi- St. + -pi-

Eg. arupita

2, Tenses:

1. Past: - $t-,-t t_{-},-n t-,-i-$

2. Future: -p- St. \pm Cau. + T.M.

Eg. ceyta

arutta

koţupitoon

koḷuupittoon

tanta

veepoon

3. Person, Number, and Gender marker:

1. Singular: -an, aan, -oon St. \pm Cau. + T.M. + Pnag.

Eg. ku-ittavañ

ceyitaan

kofupitoon 
2. Plural: -oor

Eg. koṭi-oor

4. Participle:

1. Infinitive: -a- St. \pm Cau. \pm T.M. + Inf.

Eg. aaka

2. Relational: St. \pm Cau. + T.M. + R.P.

Eg. tanta

piranta

3. Declension of Nouns: Cases:

1. Accusative: -a (ai)

Eg. itta

2. Dative: -ka, (in genetive sense); -ke (ku)

Eg. kaalitika (to Kaaliti)

taviraike

3. Genitive: $-a, \emptyset$,

Eg. ca-atana-a

piţanokurummaka!

kooño kungru

4. Constructional Types:

1. Nominal:

Eg. matira antai visuvaan antai ceenta-a taaña

paaṇita vaạ̣ikañ nețumalaan

2. Finite:

Eg. antuvan koḷupitavan nikamatoor koți-oor

3. Participial :

Eg, kuvira antai cey-a taana

teenuur tanta koon. . . ceyitaan

kumul-uur piranta kaavuți-i

ilańkoo aaka arutta kal 
5. Concord rules:

1. Number concord:

1. Singular:

Eg. pitan ..... veepoon

vaṇikan elacantan

2. Plural:

Eg. nikamatoor koṭi-oor

2. Gender concord:

Eg. ceyitaan tacan ciruvan

kurummakal kiiran noori

5. Text and translation of the inscriplions

\section{Mankulam}

1. kaniy nanta-a siriy-i

kuvanke dhammam i

tta-a neluñcaliyan pa

na-an katala-an valutti

y kolfuupitta-a pali-iy

Charity (given to) the auspicious Kuvan the great Jain monk. This abode was caused to be given by Katalan valutliy, a servant of Nețuncaliyan.

2. kaṇiy nanta siriy ku-an

dhamam ita nețiñcaliyan saalaakan

ilañcatikan tantai cațikan

ce-iya paliy

Charity of auspicious Ku-an, the great Jain monk. This abode was done by Cațikan the father of Ilañcațikan and brother-in-law of Netiñcaliyan.

3. kaṇi-i nata siriy kuva

vel-araiy nikamatu

kaaviti-iy kaalitika antai

asutan pinava koțpitoon

This/here is the auspicious Kuva the great Jain monk. Asutan pinava the father of Kaaliti the merchant chief of the guild from Vel-araiy caused to give. 
4. cantaritan koțupitoon

Cantaritan, who caused to give.

5. vel-arai nikamatoor koṭi-oor

Those who gave were the merchantile guild of Velarai.

\section{Tiruvatavur}

6. paanklkata ari-itan kottuupitoon

This was caused to be given by Aritan of Paankaatu.

7. uupaacan para-acuu

uurai koṭuupitoon

The abode was caused to be given by the devotee Paraacuu.

\section{Konkarpuliyankulam}

8. kura koṭupitavan uupare-an uuparuvan

The roof was caused to be given by the devotee Uuparuvan.

9. kura koțala ku-ittavan coora aatan-een

He who thatched the mat for the roof was I, Coora antan.

10. paakan-uur peeraatan pilan ilta veepoon-n

Peeraatan pitan of Paakanuur is he who will thatch this.

11. uupaaca-an toṇțu

\section{Kilavalavu}

lavañ koṭu pạ̣i-i

The abode was given by the devotee who has duties.

\section{Vikkiramankalam}

12. antaiy pikan makan cey-a taaña

Charity was done by the son of the great man Pikan.

13. em uur ca-atana-a taaña

Charity of Caattan of our village.

14. antai ariyti

\section{Mettupatti}

The great man Ariyti. 
15. antai iravaatan

The great man Iravaatan.

16. matira antai visuvaan

The great man Visuvaan of Matira.

17. antai ceenta-a taana

Charity of great man Ceenta.

18. ceentantai cantan

Cantan (son of) the great man Ceenta.

19. tịi-il-a taana

Charity of him who is of Tifi-il.

20. patina..r atai

The great man of Patina.. r.

21. kuvira antai cey-a taaña

Kuvira, the great man, who has done the charity.

\section{Cillannavacal}

22. ee-umi naaţu kumul-uur piranta kaavu|̣i-i tenku cirupoocil ilayar ceyta atị̧a-anam

The merchant chief, who was born at Kumul-uur in the country Ee-umi, (for him) the abode was done by the attendants of south Cirupoocil.

\section{Karunkalakuti}

23, eelaiy uur aritin pali

The abode of Aritin of Eelai-uur.

\section{Alakarmalai}

24. mattiraiy pon kolavan aatan-a taana

Charity of Aatan the goldsmith of Mattiraiy.

25. maa taviraike uupu vaaṇikan viyakan kanatikan

To the great lady devotee/disciple, by the salt merchant Viyakan kaụatikan. 
26. saapamila-in pomitti

(To) Saapamita the num.

27. paanita vaaṇikan neţumalaan

'The candy seller Nefumalaan.

28. kolu vạ̣ikan elacantan

The ploughshare merchant (smith) Elacantan.

29. vaaci kalumaaran taana

The resident, Kalumaaran's charity.

30. taar-aṇi-i kolupita-avan

Taar-ani, who caused to give.

31. kaasapan-a tavira-a

Kaasapan, the spiritual teacher.

32. avaruum kutupitoo

He also caused to give.

33. veenpali-i aruvai vaaṇikan ela-a ațan

Ela atan, the cloth merchant of Veeupali.

\section{Tirupparankunram}

34. antuvan koţupitavan

Antuvan who caused to give.

35. erukaațur iịa kutumpikan

poolaalaiyan ceyta aay cayan netucaatan

This was done by Poolaalaiyan, a husband/man of the tree-climber caste of Erukaatur. (The occupants) Cayan, the Aay, Netucaatan.

\section{Mutlupalli}

36. vintai-uur caiya-alan kaaviy

The abode of Caiyalan of Vintai-uur.

\section{Anaimalai}

37. iiva kuñratuu-uraiyul paatanatan taaña eri aritan attuvaayi arața kaayipan 
These are the charities of Paatanatan the resident of Kungratuur. (The occupants) Eri, Aritan, Attuvaayi, Arața, Kaayipan.

\section{Pukalur}

38. taa amạnan yaarruur ceñkaayapañ uraiy

koo aatan ceellirumporai makan

perunkałunkoon makan[i]lan

katunkoo[i]lańkoo aaka arutta kal

(This) is the abode of Cenkaayapan, a Jain monk of Yaarruur. This stone was cut while Ilankatunkoo, the son of Perunkatunkoon, the son of the King Aatan Cee[ra]llirumporai, was becoming the heir apparent (prince).

39. aman... yaarịu . cenkaa.....

koo aa... .llirumpurai makan perun

kaţuńkoon makañ kațunkooñ[i]lankaţuń

koo[i]lankoo aaka arupita kal

This stone was caused to be cut while Katunkoon Ilankatunkoo, the son of Perunkatunkoon, the son of King Aa[tan Cee[ra]]llirumpurai, was becoming the heir apparent.

40. nalliyi-uur-aa piţantai makal kiiran korna

Kiiran korra the daughter of Pitantai of Nalliyuur.

41. nalli-uur-a pitan kurummakal kïran noori ceyipita pali

The abode was caused to be done by Kiiran Noori, the granddaughter of Pitan of Nalliyuur.

42. kartu-uur pon vaanikan natti atiflaanam

(This is) the abode of Natti, the goldsmith of Kartu-uur.

43. ... ṇaakan makan ilankiiran

Ilanikiiran son of . . . naakan. 
44. kaṇimaan

\section{Mamantur}

teenuur tanta koon kuñru

ceyitaan tacan ciruvan

The great Jain. 'The hill of the lord who overcame 'Teenuur.

Son of a carpenter did it.

\section{Araccalur}

45. elutu nampaṇ vattan maṇiy vaṇnakkan teevan caattan

(This is) the writing (of) the good man (friend) Vattan; Teevan caattan.

\section{Pillaiyarpatli}

46. erukaatfuruk koon perunkiiran

Perunkiiran, the lord of Erukaalfuru.

6. Tamil Brahmi inscriptions

Maańkulam

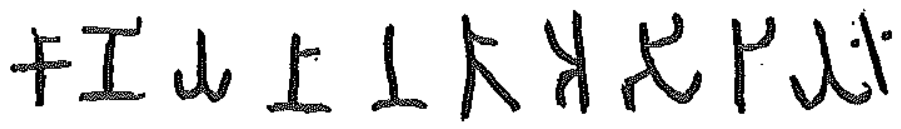

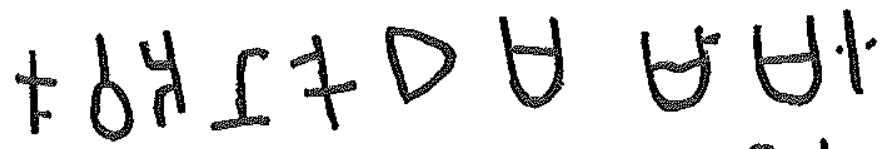

1

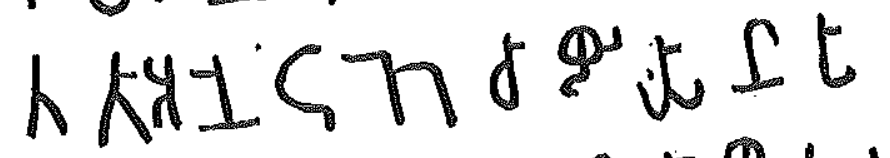

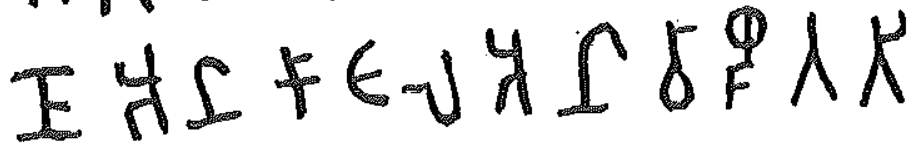

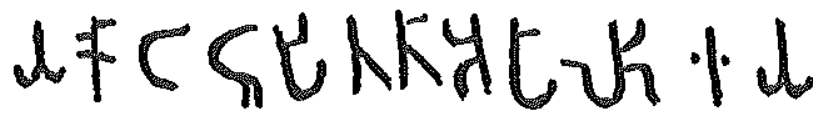


A CRITICAL STUdY OF THE TAMIL BRAHMI INSCRIPTIONS 189

扛上此仙山拄卫

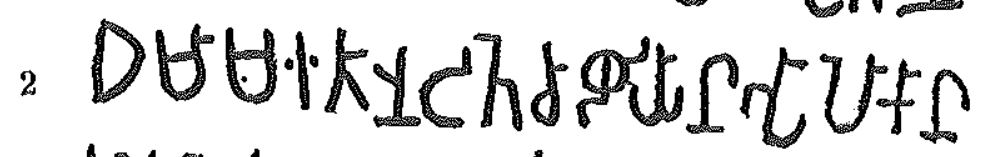

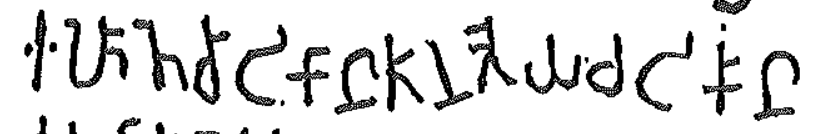
d小utron

fINKexutb

bvysuif

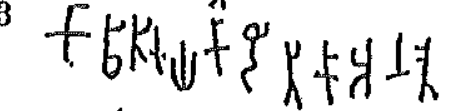

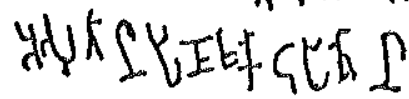

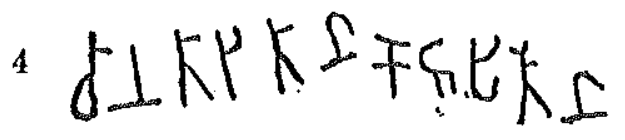

5 tน

Tiruvaatavuur

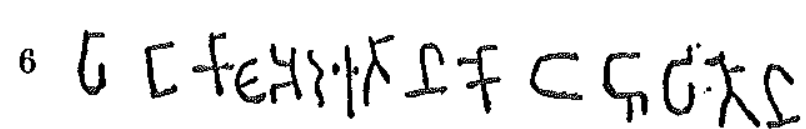


190

Etidecind

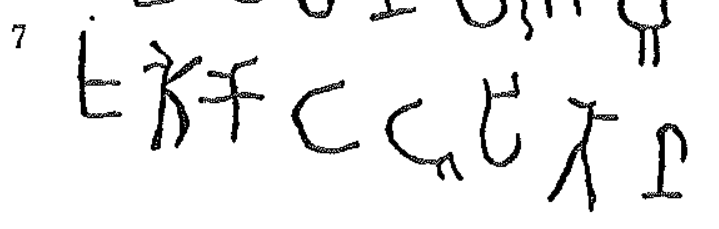

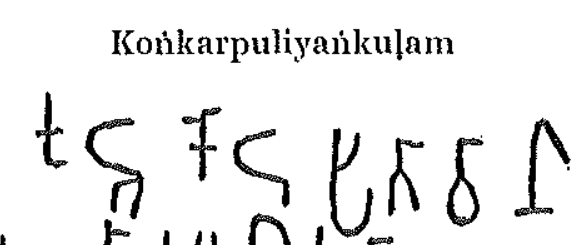

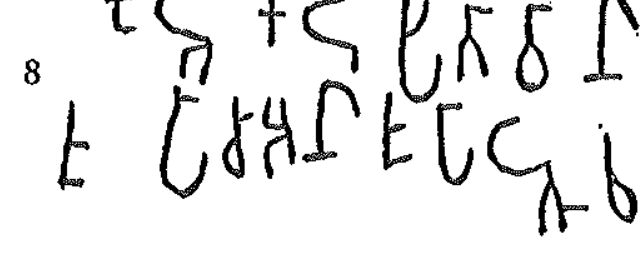

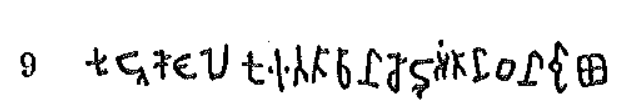

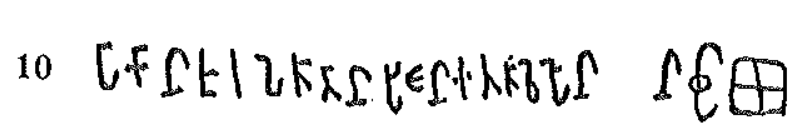

Riillavalavu

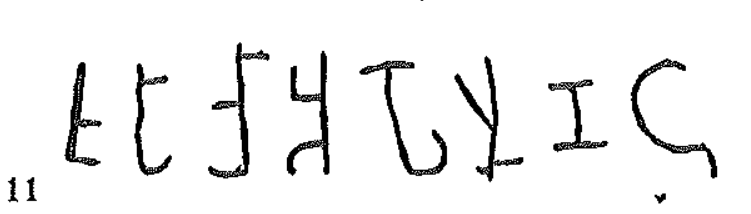

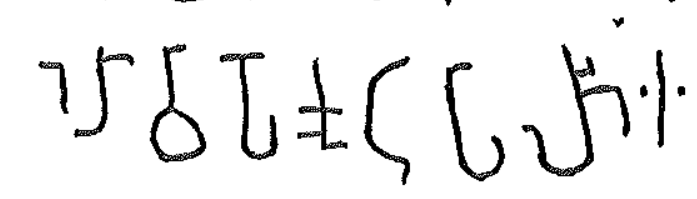


A CRITICAL STUDY OF THE TAML BRAHMI INSCRIPTIONS

Vikkiramankalam

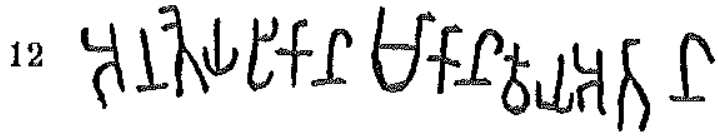

13

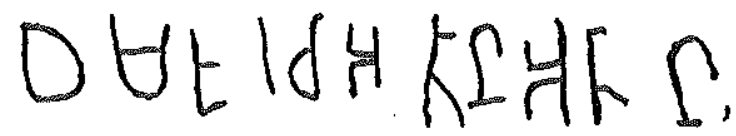

Meețuppaṭ̣i

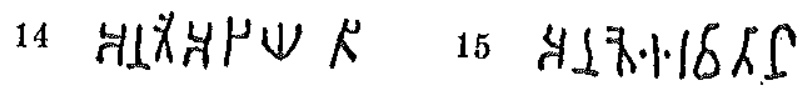

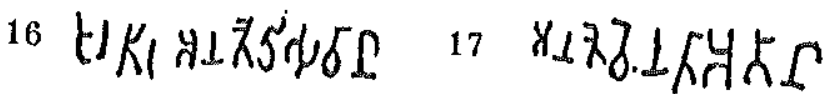

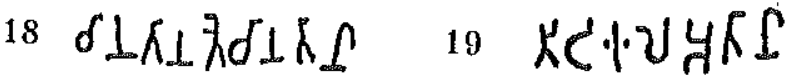

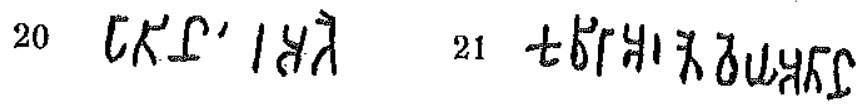

Cittannavaacal

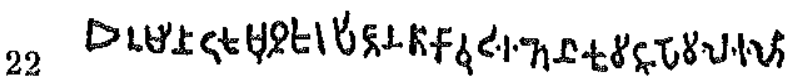

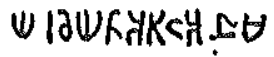

13 Acta Orientalla, XXXIV 
Karuńkaalakkuṭi

$230 \%$.

Alakarmalai

$24 \forall K K$ U.

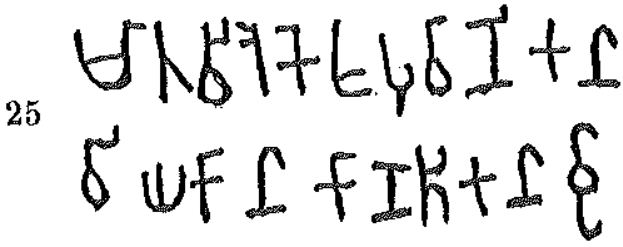

26 AUHK०II

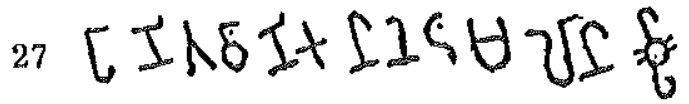

$28+8, I+P D$ und 1 S

29 atophan 
CRITICAL STUDY OF 'THE TAMLL BRAHM INSGRIPTIONS 193

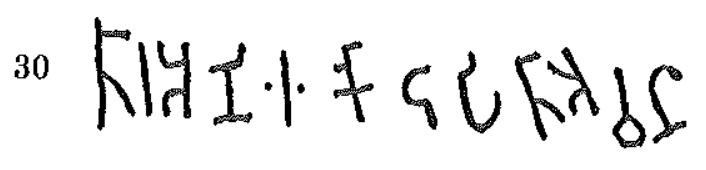

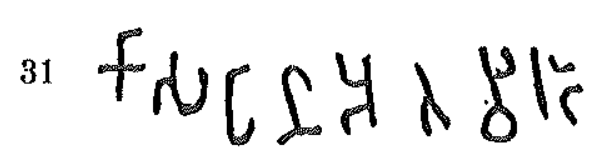

32 4640 t5

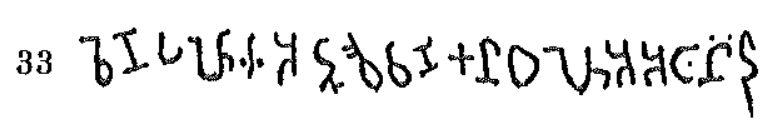

'Tirupparan̉kuñram

34 4IL6IF50 65

${ }^{35} \frac{\Delta+f(1=-g t c \theta \tau+\rho}{2 \tau}$

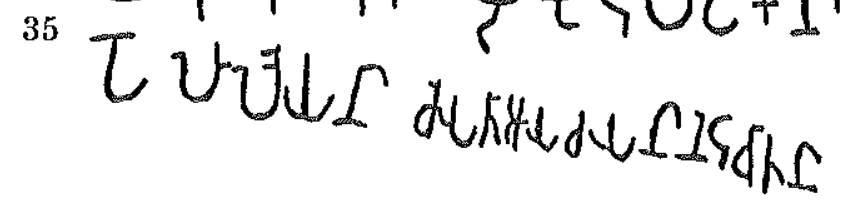

as 61 Re 
194

R. PANNETERELYAM

Aanaimalai

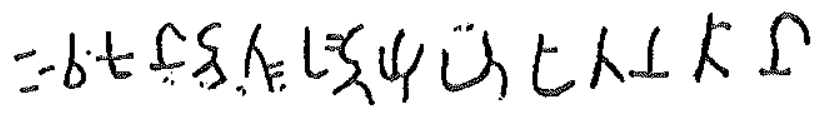

37

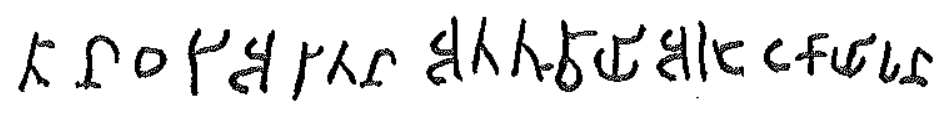

Pukajuur

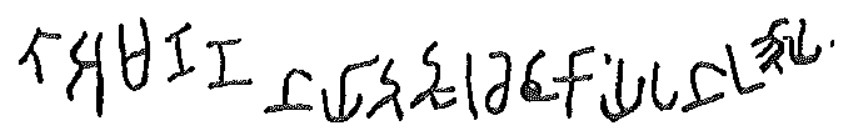

38

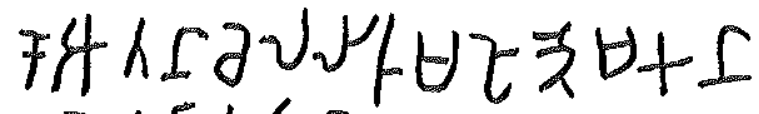

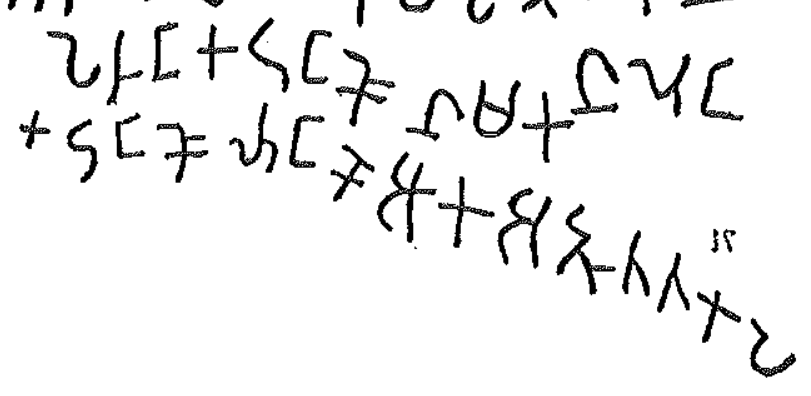

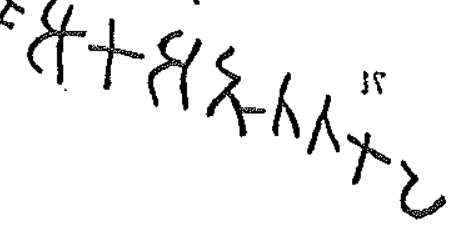

A甘T

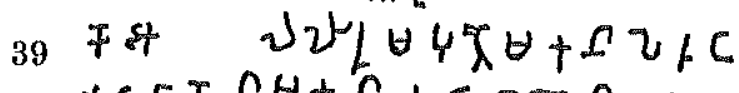

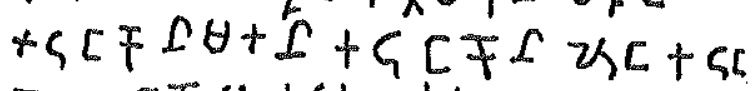

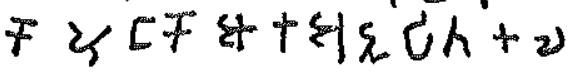

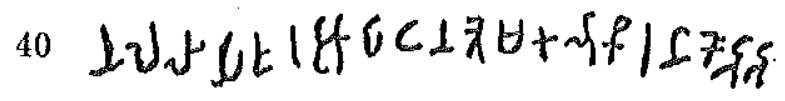




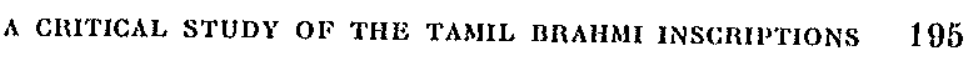

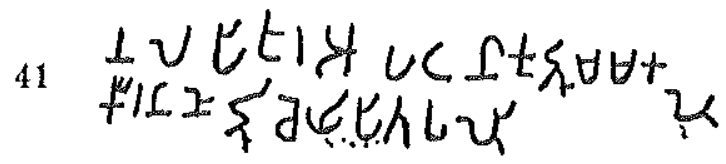

$+H-1 T \Omega 5 I+\infty$
INKSKRCESH

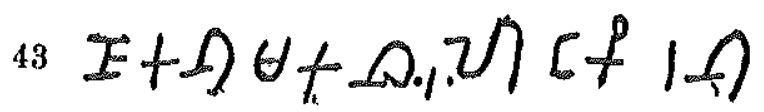

$* T \gamma=$

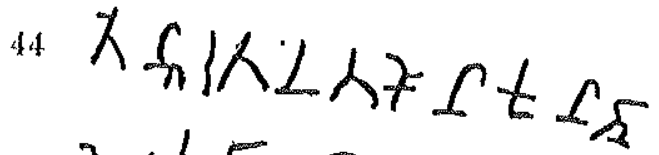

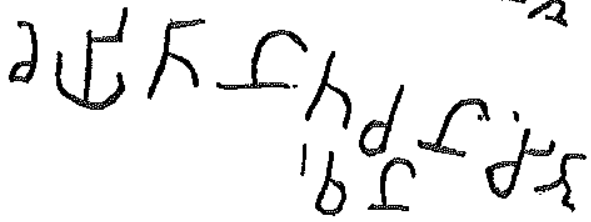

Araccaluur

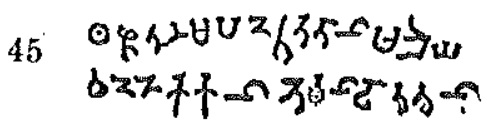


Pillaiyaarpatti

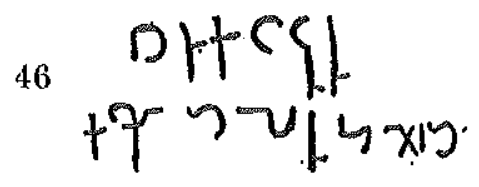

Conversion table:

$\begin{array}{cccc}\text { R.P.S. } & \text { I.M. } & \text { R.P.S. } & \text { I.M } \\ 1 & 1 & 27 & 37 \\ 2 & 2 & 28 & 38 \\ 3 & 3 & 29 & 39 \\ 4 & 5 & 30 & 40 \\ 5 & 6 & 31 & 41 \\ 6 & 7 & 32 & 42 \\ 7 & 8 & 33 & 43 \\ 8 & 10 & 34 & 48 \\ 9 & 11 & 35 & 51 \\ 10 & 12 & 36 & 52 \\ 11 & 9 & 37 & 55 \\ 12 & 13 & 38 & 56 \\ 13 & 17 & 39 & 57 \\ 14-21 & 18-24 & 40 & 58 \\ 22 & 27 & 41 & 59 \\ 23 & 28 & 42 & 66 \\ 24 & 30 & 43 & 73 \\ 25 & 33+34 & 44 & 71 \\ 26 & 36 & 45 & 72 \\ & & 46 & 75\end{array}$

R.P.S. - R. Panneerselvam. I.M. = Iravatham Mahadevan,

Select bibliography

Mahadevan, I. 'Corpus of the Tamil Brahmi Inscriptions', Seminar on Inscriplions, Madras, 1968.

Mcenakshisundaram, T. P. A History of Tamil Language, Poona, 1965. Panneerselvam, R. 'An Important Brahmi Tamil Inscription: a re- 
PAINTED IN DENMARK DY BIANCO LUNO A/S 


\section{TABLE OF GON'TENTS}

Hans Jürgen KonnrumpF: Das dänische Konsulat in Smyrna. Eine osmanische Urkunde aus dem Jahre $1889 \ldots \ldots \ldots$. 5

Stig Wikander: B $\alpha p z o x \alpha p \alpha \ldots \ldots \ldots \ldots \ldots \ldots \ldots \ldots$

Schuylen Jones: Demuta, Folk-Hero of Nisheigrom: Myth and Social Structure.................. 17

Lennart Enelberg: Some Paruni Myths and Hymns..... 31

Klaus Mylıus: Der zweite Adhyāya des Āśvalāyana-Śrautasūtra, erstmalig vollständig übersetzt und erläutert . . . . 95

R. Pannegrselvam: A Critical Study of the Tamil Brahmi Inscriptions $\ldots \ldots \ldots \ldots \ldots \ldots \ldots \ldots \ldots \ldots \ldots \ldots \ldots \ldots$

W. W. SchuнмлснеR : Beiträge zur Synchronie und Diachronie austronesischer Sprachen ...................... 199

1) Zu verdeckten Kategorien der Bälau-Sprache ......

2) Der Samoanische Reflex auf Urpolynesisch/ptk? mny/

3) Fijian dua 'one': rua 'two' .................

4) 'Lizard' in Bellonese ...................

5) Uraustronesisch $s-$ ein neues Phonem ..........

Book Reviews ...................... 213

Books sent to the Editor . . . . . . . . . . . . . . 229 


\section{DAS DÄNISGHE KONSULAT IN SMYRNA}

Eine osmanische Urkunde aus dem Jahre 1889

VON

\section{HANS-JÜRGEN KORNRUMPF}

Im Privatbesitz der Hamburger Bankierfamilie Brinckmamn (Brinckmann, Wirtz u. Co.) befindet sich eine Urkunde des osmanischen Sultans 'Abd ul-Hamid II, aus dem Jahre 1889 mit dem Exequatur von Carl Christian Brinckmann, dem Grossvater des gegenwärtigen Firmeninhabers, als Kgl. Dänischer Konsul in Smyrna (Izmir).

Carl Christian Brinckmann (geb, am 23.3.1853) war Staatsangehöriger des Deutschen Reiches und bereits vor 1889 als Kaufmann in Smyrna ansässig gewesen, Ev übte seine konsularische Funktion bis zu seinem Tode am 18.3.1898 aus, wurde zunächst in Smyrna beigesetzt und seine sterblichen Überreste in den dreissiger Jahren dieses Jahrhunderts nach der Familiengrabstätte in Aumühle bei Hamburg überführt ${ }^{1}$.

Seine Vorgänger in Smyrna waren holländische Staatsangehörige gewesen: von Januar 1856 bis Mai 1889 John Robert de Jongh, vor ihm sein Vater Jean de Jongh. Der Sohn von John Robert, John Atkinson de Jongh, wurde 1877 zum dänischen Vizekonsul in Smyrna ernannt. Er war ebenfalls holländischer Staatsangehöriger, aber dänischer Protégé. ${ }^{2}$

1 Die persönlichen Angaben verdanke ich Herrn Dr. C. Brinckmamn.

${ }^{2}$ Mitteilung des Kgl. Dänischen Aussenministeriums an mlch vom 28.8.1969. - Wie mir das Kgl. Niederlandische Aussenministerium (Archivar J. A. H. Nifhof) unter dem 27.11.1969 schrieb, hatten die Niederlande eine elgene konsularische Vertretung in Smyrna: Generalkonstul J. van Lennep von 1825 bis 1855 , Konsul R. J. van Lennep von 1855 bis etwa 1891 . 
Die Urkunde selbst hat ein Format von ca. $56 \mathrm{zu} 103 \mathrm{~cm}$ und ist auf starkem hellbraunem Papier in Dīvāni-Ğelī-Schrift geschrieben, wobei die Zeilen mit schwarzer, goldener und roter Tinte abwechseln. Ihre äussere Form ist die des traditionellen Fermans, da sie die Tugirã (nit roter Tinte geschrieben), Einleitungsformel, Narratio (ohne ibläg), Dispositio, Sanctio, Angabe der Beglaubigungsmittel sowie Datierung und Ortsangabe (letztere kaum leserlich) enthält; eine Invocatio über der Tugiga fehlt. ${ }^{3}$ Der Wortlaut folgt eng ähnlichen Urkunden. ${ }^{4}$

\section{Translipiption :}

'Abd ül-Hamĩd bãn bin 'Abd ül-Meğĩd muẓaffer dă’imã

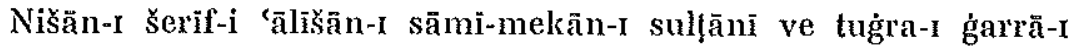
ğihān-sitān-ı bāḳänì hüükmü oldur ki :

Danimarka devleti tüğğār ve teba'asından Izmire gelüb gidenleriñ ümūr ve buşūşāt-I văḳı'a-r tiğāretlerini rü’yet eylemek üzere konsolos bulunan Yān dö Yonganñ̄ vuḳū vefātına mebnī yerine bu kere Almanya devleti teba'asmdan išbu rãfici tevkị -i

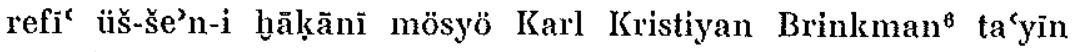
olundığı beyāniyle

s Nach Zajaczkowski-Reychman: Zarys dyplomatyki osmańsko-turecklej (Warszawa 1955), S. 73 ff.; M. Guboglu: Paleografia şi diplomatica turco-osmană (Bucureşli 1958), S. 53 fr.; F. Kraelitz: Osmanische Urkunden in tirkischer Sprache aus der zweiten Hälfte des 15. Jahrhunderts (Wien 1921), vor allem S. $11 \mathrm{fl}$. Dle vorliegende Urkunde bezelchnet sich selbst als nțān-1 hümäyūn. Ebenso verhält es sich mit dem Exequatur für den österreichisch-ungartschen Generalkonsul in Alexandria 1877, von Z. Veselá-Pfenostlová im Archiv Orientálni 37,1 (Praha 1969), S. 12-18 veröfientlicht. Dass dle Invocatio dort abgeschitten worden ist, scheint mir fraglich, da sie auch bei dem hier besprochenen vollständigen Dokument fehlt, und M. Guboghu, op. cit. eine Rethe von Urkunden mit Tugraa und olne Invocatio vorstellt (vgl. Facs. 159, 160, 175, 187, 192, 196, 200).

- Vgl, etwa den eben erwähnten Aufsatz: Le décret Impérial (nişâni hümâyûn) délivéé pour le consul général de l'Autriche-Hongrie à Alexandrie von $Z$. VeseláPrenosilová.

s Der Name ist am Anfang verschrieben: ه 4 grosilj; vgl. a. das Osmanische Statistische Jahrbuch (Sāmāme) für 1302/1884f, S. 615.

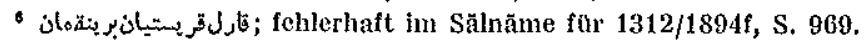




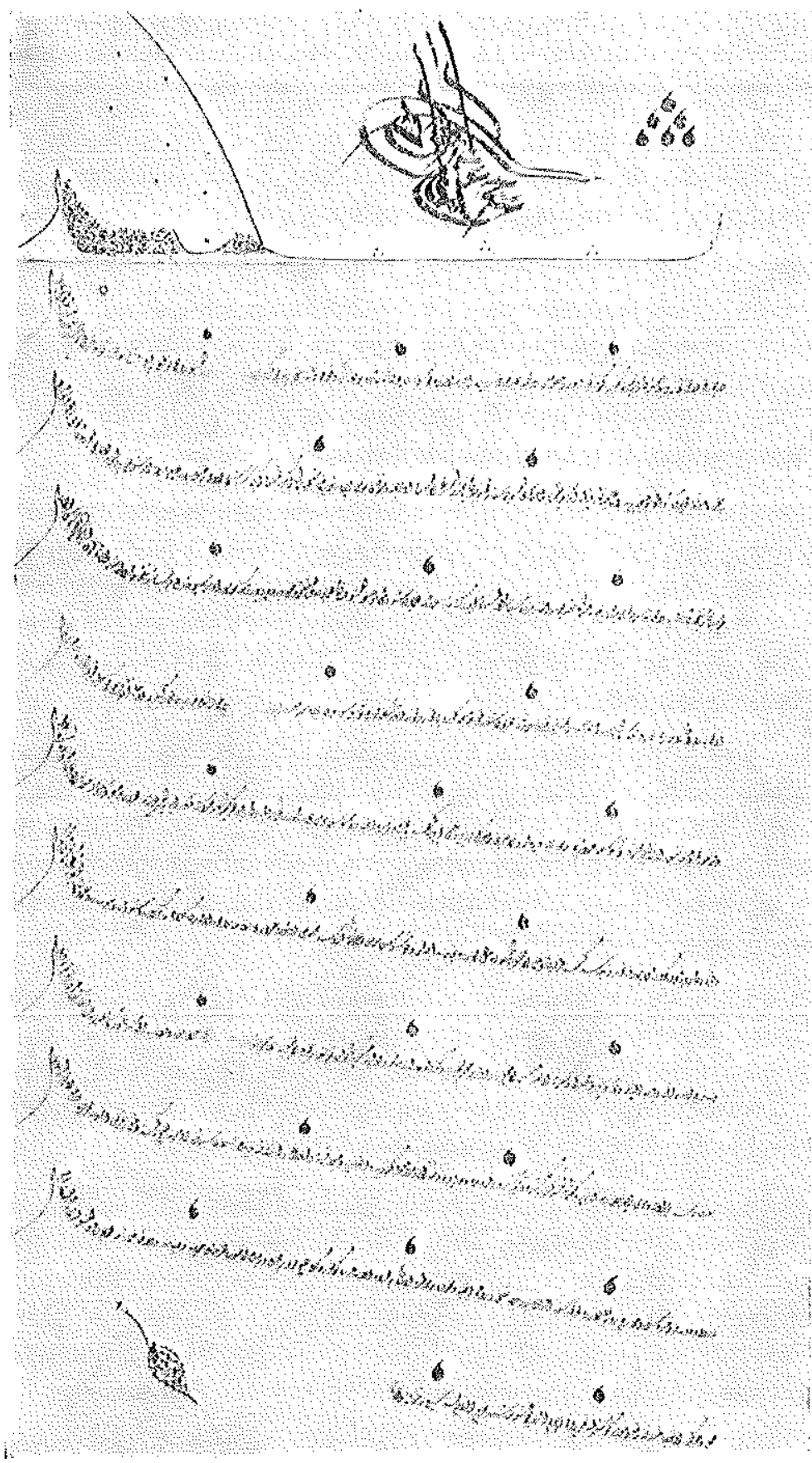


sābrḳı ve 'ahdnäme-i hümāyūn šürütı mūğibinğe yedine berät-ı

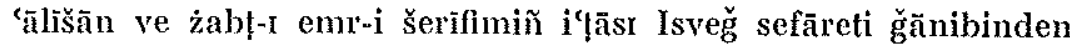
bä-taḳrïr istid ‘ă olummuš oldiğma ve mütevefä-I mümā-ileyhe me’mŭrīyetini mutażammm biñ ikiyüz yetmiš iki senesi evā'il-i Reğebinde berñt-ı 'ālĩ ve żabt-I emr-i šerîf verilmiš oldığ kuyüddan ve mümä-ileyh mösyö Karl

Kristiyan Brinkman Almanya devleti teba'asmdan oldığı tābi'îyet ḳaleminiñ taşdị ve išăretinden müstebăn olmuš ediğine bină'en 'ahdnāme-i hümāyūn šürūị mūğibinğe išbu nišăn-r hümāyūn-r mevhibet-makrnūnumu verdim ve buyurdum ki: Mümā-ileyh mösyö Karl Kristiyan Brinkman devlet-i mǚšãrileyhe tarafmdan Izmirde konsolos olub

ol ğănibe varan Danimarka tüğğăr ve teba'asmıñ ümūr ve

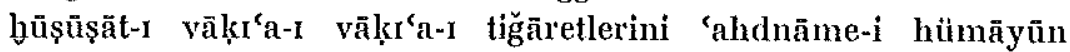
šürūṭ mūğibinğe görüb tüğğår-r merḳūma ümūr-r müškilelerinde konsolos-r mümä-ileyhe mürñğa 'at edeler, ve Danimarka bayraġ altunda yürüyen tüğğăr gemileriyle dāḩil oldrğı gibi ‘ãdet-i kadime üzere meta'larmiñ

defter șüreti mūğibinğe ta'yin olunan elğileriñ ve konsolosluk 'avä'idini mümā-ileyhe vereler', ve adamlarm ahariñ borğ ve töhmeti ičün kimesne renği̊de ve remïde eylemiye, ve konsolos-r mümā-ileyden dört biñ akceden ziyāde her kimiñ da'väsı zuhür eder ise 'ahdnäme-i hümăyün müğibinğe Der-Sa'adetime havāle olunub gayrì yerde istimā' olunmrya,

ve karadan ve deryãdan gelen Danimarka tüğğăr ve teba'asma kimesne tarafindan dabl olunmayub āsüde-hăl ïzere tiğäret eyliyeler, ve konsolos-I mümä-ileyhiñ kendüsine malıșūş olub miḳdāri niẓămına muvāfı̣̣ olarak gelen me'kūlāt ve mešrūbāt ve melbūsăt ve mefrūšătı iskeleye gelür ise gümrük

ve sã’ir nām ile bir akče ve bir habbe almryalar, ve konsolos elğileriñ vekilleri olmaggla habs olunmayub ve evleri mühürlenmeyib ve gezülerin aramryalar, ve evlerine 'askerīden konak konulmag̉la renğîle olunmayub og̉ullarr ve müste'min bidmetkärlar 'avāxrż ve ḷașşābīye akčesinden ve resm-i mașdarīyeden ve tekälîf-i ‘örfĩyeniñ ğümlesinden 
mưăf ve sālim ola, ve mümā-ileyh ba`ż-r zamānda bì yere gitmek istedilkde gelišde ve gidišde ve karada ve deryāda ve menāzil ve merālịilde geziiye ve eșvāb ve hayvānlarına ve emvãl ve erzālunna ve yammda olan müste'min adamlarma hič bir kimesne dabl ve ta‘arruż etmeyib her kanda dābil olur ise zäd

ii zevãde ve sä’ir zabīresini dahi narb-r rūzì üzere akčesiyle aldrkda bir veğhile ta'allül ve nizā' etmiye, ve mahüf ve mubātara olan yerlerde älāt-I ḷarb götürdükde hïč ferd renğide ve remìde etmeyib her liălde himāyet ve şıānet ve dä'imã 'ahdnāme-i hümāyün ve išbu nišān-I 'ālišānm müğibinğe

'amel ve hareket olunub bुilăfma riżã ve ğevãz gösterilmiye. Taḥrỉen fỉ 'l-yevm ir-rābi' 'ašrr min šehri Muḷarrem il-ḥarām sene seb $^{c}$ ve selās-miye ve elf

Bi-makăm Ḳostaṇ̣inìye il-maḷrüse il-maḥmiye

\section{Übersetzung:}

'Abd ul-Hamid Hăn, Sohn des 'Abd ül-Meğĩd, immer siegreich. Der Befehl des ehrwürdigen, erhabenen und erlauchten sultanischen Dekrets (nišăn) und der glänzenden, welterobernden Tuğrā ist:

Vonseiten der schwedischen Botschaft wurde mitgeteilt, dass Jean de Jongh gestorben ist, der Konsul gewesen war, um sich der anfallenden geschäftlichen Probleme der dänischen Kaufleute und Untertanen, die Izmir besuchen, anzunehmen, und dass nunmehr an seiner Stelle der deutsche Untertan Herr Carl Christian Brinckmann, der Inhaber dieses erhabenen kaiserlichen Handzeichens (tevkin ), ernannt wurde. Es wurde durch eine schriftliche Note ersucht, ihm gemäss seinem Vorgänger und den Bedingungen des kaiserlichen Gesetzes" Meinen erhabenen

\footnotetext{
" Das erste Handelsabkommen zwischen dem Osmanischen Reich und Dänemark(-Norwegen) geht auf den 14.10 .1756 (frz. Text bel G. Noradounghian: Recuell d'actes internationaux de l'Emplre ottoman I (Paris etc. 1897), S. $308 \mathrm{ff}$.) zurack und enthält auch die einschägigen Bestimmungen tber dänische Gesandte und Konsuln in der Tirkel, die von den mit anderen Staaten abgeschlossenen
} 
Berat und das kaiserliche Exequatur zu gewähren. Aus den Registern erweist sich, dass dem genannten Verstorbenen in der ersten Dekade des Reǧeb $1272^{8}$ ein erhabener Berat und ein kaiserliches Exequatur über sein Amt ausgestellt worden waren. Vom Ausländeramt ${ }^{2}$ wurde beglaubigt und angezeigt, dass der erwähnte Herr Carl Christian Brinckmann deutscher Untertan ist.

Deshalb erteilte Ich gemäss den Vorschriften des kaiserlichen Gesetzes dieses mit Meiner kaiserlichen Grossmut verbundene Dekret und befahl:

Der genannte Herr Carl Christian Brinckmann sei in Izmir Konsul des erwähnten Staates. Er nehme sich der anfallenden geschäftlichen Probleme der dorthin kommenden dänischen Kaufleute und Untertanen nach den Bedingungen des kaiserlichen Gesetzes an, und die besagten Kaufleute sollen sich mit ihren Schwierigkeiten an den genannten Konsul wenden.

Unter Einbeziehung der unter dänischer Flagge fahrenden Handelsschiffe sollen sie nach altem Brauch gemäss der Abschrift ihrer Warenbächer die fesigesetzten Abgaben für die Botschafter und das Konsulat dem Genannten geben.

Niemand darf seine Leute wegen der finanziellen oder sonstigen Schtld eines anderen behelligen.

Wenn sich bei dem erwähnten Konsul ein Prozess von irgend jemandem mit mehr als 4000 Akče (Streitwert) ${ }^{10}$ ergibt, wird er nach dem kaiserlichen Gesetz an Meine Glückliche Pforte überwiesen und darf an anderer Stelle nicht verhandelt werden.

Kapitulationen kaum abweichen. Vgl, auch I. H. Uzunçarşılı: Osmanlı tarihi IV, 2 (Ankara 1959), S. 244. Z. Veselá-Prenosilová konnte Grtndelemente des Textes fitr thre Urkunde in dem bei Ferīūn: Münse’āt üs-setāṭin II, $473 \mathrm{ff}$. der 2. Auflage (Istanbul $1275 / 1858 \mathrm{f}$; II, $381 \mathrm{ff}$. der 1. Auflage Istanbul 1265/1848 f.) abgedruckten 'Ahdnăme des Sultans für die britlsche Könlgin Elisabeth aus dem Jahre 1010/1601 $f$. nachweisen, was natürlich auch für unser Dokument gill.

${ }^{8}$ Entspricht dem 8.-17.3.1856.

* Täbi'iyet ḳalemi = Amt für Staatsangehörigkeit; Ausländeramt beim osmanischen Aussenministerfum; M, Z. Pakalm: Osmanlı tarih deyimleri ve terimleri sözlìğï (Istanbul 1946-55).

10,4000 Akče Streftwert waren die ubliche Grenze in den Kapitulationen. Her wie auch im folgenden trägt der Urkundentext den tatsächllchen Gegebenheiten in der zweiten Hälfte des 19. Jahrhunderts (Konsulargerichte, gemischte Handelsgertchte, andere Währung, geändertes Steuersystem usw.) kaum Rechmung. 
Niemand darf die zu Land und zu Wasser kommenden dänischen Kaufleute und Untertanen bedrängen, und sie sollen ungestört Handel treiben.

Wenn für den genannten Konsul selbst bestimmte und mengenmässig ordnungsgemäss eintreffende Esswaren, Getränke, Bekleidung und Möbel an die Anlegestelle kommen, darf man keinen Akče und nicht den geringsten Betrag als Zoll oder unter anderem Namen erheben.

Die Konsuln sind Vertreter der Botschafter; sie dürfen deshalb nicht inhaftiert, ihre Häuser nicht versiegelt und ihre Ausflüge nicht kontrolliert werden.

Sie dürfen nicht durch die Einquartierung von Soldaten in ihren Häusern belästigt werden, und ihre Söhne und schutzbefohlenen Bediensteten sollen von der Kriegssteuer, ${ }^{11} \mathrm{dem}$ Schlachtgeld ${ }^{12}$, der Zusatzabgabe ${ }^{13}$ und den anderen Sondersteuern's befreit sein.

Wenn der Genannte gelegentlich einen Ort besuchen will, darf niemand auf der Hin- oder Rückreise, zu Land oder zu Wasser, bei den Halteplätzen und Poststationen seinen Reiseweg, seine Waren, Tiere, Besitztümer, Vorräte und die bei ihm befindlichen schutzbefohlenen Bediensteten bedrängen oder belästigen.

Wohin er auch immer kommt, man darf in keiner Weise Vorwände suchen oder mit ihm streiten, wenn er seine Wegzehrung und andere Vorräte nach dem Tageskurs bar bezahlt.

Niemand darf ihn behelligen, wenn er an gefährlichen Orten Kriegsgerät mit sich führt.

\footnotetext{
11 'Avărı̌, auch 'avärı̇̇-ı divānìye: eine Sonderstewer, vor allem Kriegssteuter, dle im Laufe der Zeit zu einer ordentlichen Steuer wurde; M. Z. Pakalı, op. cit.; vgl, auch A. Sućeska: Die Entwicklung der Besteuerung durch dle 'Avâriz-i divanifye und die Tekâlif-i 'orfíye im Osmanischen Reich während des 17. und 18. Jahrhunderts, in: Suddost-Forschungen 27 (Munchen 1968), S. 89-130.

12 Kaşşăbiye akčesl: Schlachtgeld der Metzger, eine traktatwidrige Umlage auf Häute; J. Th. Zenker: Türklsch-arabisch-persisches Handwörterbuch (Lelpzig 1866-76).

1s Resm-1 maşdariye: eineinhalbprozentige Zusatzsteuer auf den Zoll in Konstantinopel für bestimmte Waren [Bianchi-Kieffer: Dictionnaire turc-françals (Parls 21850; danach J. Th. Zenker, op. eit.), besonders alkoholische Getränke;

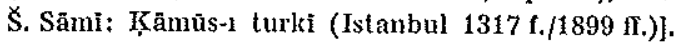

14 Tekāliff-i 'örfìye: in Kriegszeiten zulässige Sondersteuern, die 97 an Zahl erreichten; M. Z. Pakalın, op. cit.; vgl, auch A. Sućeska, op. eit.
} 
Er soll in jedem Falle geschützt und bewahrt, es soll immer nach dem kaiserlichen Gesetz und diesem Meinem erhabenen Dekret verfahren und keinerlei Zuwiderhandlung zugelassen werden.

Geschrieben am 14. 'Tage des heiligen Muharrem im Jahre $1307 . .^{15}$

In dex geschützten und behïteten Stadt Konstantinopel.

1s Entspricht dem 10.9 .1889 . 


\section{BAPZOXAPA}

PAR

\section{STIG WIKANDER}

Dans Anatolian Studies vol, XVII (1967) p. 193, Richard P. Harper vient de publier une inscription grecque de l'époque impériale, dédiée à la déesse Anaitis. Elle a été trouvée à Ortaköy, non loin de Niğde, done dans l'anciemne Cappadoce. Il doit s'agir de l'inscription greeque la plus orientale qui mentionne Anaitis. Elle est mentionnée dans plusieurs inseriptions de l'Asie Mineure, presque toutes de la partie occidentale du pays, notamment de l'ancienne Lydie.

Cette inscription fait connaître une épithète inconnue jusqu'ici. On y lit:

\section{OEA METIITH ANAEITIDI BAPZOXAPA}

Les épithètes des divinités dans les inscriptions de l'Asie Mineure contiennent souvent des adjectifs géographiques. Ainsi, Artemis, souvent identifiée à Anailis, est appellée

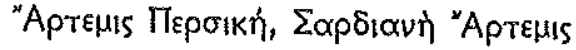

Pour expliquer $\beta \alpha \rho \zeta \circ \chi \alpha \rho \alpha$, on pense donc d'abord à Barzos, mentionné comme une ville de l'Orient romain, objet des attaques des Parthes:

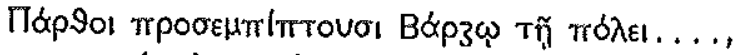

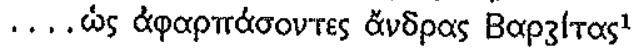

Il est vrai que l'action du roman byzantin, que nous venons de citer, se refère à un passé lontain, à peu près l'époque où

\footnotetext{
1 Niketas Eugenlanos, I, vv. 6 et 14, Erotici scriptores graecl recognovit Hercher
} II, 437. 
fut redigée notre inscription ${ }^{2}$. Cependant, la désinence $-\chi \propto \rho \alpha$ ne saurait s'interpréter comme un suffixe de dérivation dans aucune langue qui pourrait être actuelle ici. Barzos, aux resonances iraniennes et arméniennes, mais inconnu par ailleurs, doit être l'invention de l'auteur pour donner un peu de couleur locale à son roman.

On songera plutôt à un nom de personne composé, bien attesté dans la forme greeque Barzapharnēs. ${ }^{3}$ C'est le nom d'un satrape à l'époque parthe. Plus tard, il est attesté dans les traditions epico-historiques de l'Iran comme nom d'un des grands héros de l'antiquité, fils de Kai Kāüs et oncle de Kai Chosrau. TTabarì donne la forme Borzāifāh, forme déformée: l'éditeur de ce volume de Tabari, Noeldeke, a bien vu l'identité avec Ferỉborz mais donne une fausse étymologie (Țabarī I, 605 avec note f). La forme correcte se trouve ailleurs, p. ex. Moğmal al-tawārīh wa-I-qișaș (Tehran 1318) p. 29 Borzfarrī. L'auteur anonyme de cet ouvrage remarque que c'est là la forme originale que Ferdosī a changé en Feriborz pour des raisons métriques: en effet, le mutaqãrib, mètre préféré de l'épopée iranienne, est dominé par un pied qui n'admets pas la structure métrique de Borzfarrī $(--)$, pendant que Ferīborz $\left(u_{-}\right)$s'y conforme naturellement.

Le Shãhnāmch emploie souvent les deux mots farr et borz, qui forment ensemble le composé Borzfarri, composé qui continue la forme vieux-perse Barzapharnēs. Persan farr continue donc vieux-perse farnah, ancien mot qui désigne le symbol de la dynastie légitime de l'Iran, une sorte de gloire ou auréole. Le mot correspondant en avestique, $x^{v} a r \check{r}_{n} h$, fait part de plusieurs épithètes divines; on dit d'Anähitã qu'elle 'possède tant $\boldsymbol{x}^{v}$ arenah que toutes les eaux ensemble' (Yasht V, 96).

Mais une forme grecque $\beta \alpha \rho 3 \circ \chi \alpha \rho \alpha$ peut-elle correspondre aux formes iraniemmes que nous venons de discuter?

Nous avons a faire avec une inscription de Cappadoce, non loin du domaine linguistique de l'arménien. Or, dans le panthéon de l'ancienne Arménie pré-chrétienne, la divinité la plus importante est la déesse Anahit, empruntée à la mythologie iranienne,

2 'Eine ziemlich versehwommene heidnisch-hellenische Vergangenhelt' dit Krumbacher, Gesch, d. byz. Literatur 763 du milieu de Niketas.

${ }^{3}$ Flave-Josephe, Antlquites XIV, 23 suiv, Bellum judaicum I, 11. 
la même qui se trouve souvent comme Anaitis dans les inscriptions grecques de l'Asie Mineure.

En analysant la forme $\beta \propto \rho 3 \circ \chi \alpha \rho \alpha$, on peut discuter deux alternatives: qu'elle représente une forme arménienne ou que l'arménien, qui a reçu tant d'emprunts de l'iranien, nous aidera a donner une iclée des différentes formes dialectales iraniennes que peuvent montrer les cmprunts faits à l'iranien par beaucoup de langues du Proche Orient.

Or, en arménien, le phonème vieil-iranien $x^{v} a-/ f a$ - se présente de trois manières diverses:

1. vieux-perse farnah donne le nom phar-kh 'gloire' et le nom de personne Barzaphran (grec Barzapharnës), la labiale sourde aspirée se substituant à la fricative labiale;

2. $x^{*} a$ - initial en avestique a donné $x o$-dans toute une série de noms de personne; 5

3 . Entre voyelles, avestique $-x^{v} a$ - a donné arménien $x a$, donc kaxard de kaxvarétha 'sorcier'. ${ }^{6}$

Grec - $\chi \propto \rho \alpha$ peut donc rendre un $x^{v} a r r$ moyen-iranien avec la désinence grecque $-\alpha$.

Quant au premier membre du composé, son étude demande une analyse de tous les noms de personne en -farnah, étude que j'espère donner sous peu. Elle montrera que presque tous présentent comme premier membre un mot avec le sens 'lumière éclat' etc. Barzo- doit donc représenter une forme iranienne du várcas védique, splendeur, éclat', signification qui persiste quoique souvent méconnue -, en avestique varěcah, pehlevi varc, persan varğ: une variante se retrouve dans les noms propres comme Borzfarli-Feriborz et n'a rien à faire avec borz-buland 'haut'.

(Dans KZ 84, 1970, 207 suiv. M. Rüdiger Schmitt vient de proposer une autre explication qui n'emporte guère la conviction: il y voit le nom de la montagne Alborz, avestique Harä běrĕzaitī, déformée d'une facon singulière. Mais l'ordre des deux mots Harā-berezaitī n'est jamais r'enversé).

\footnotetext{
s Hübchmann, Armentsclie Grammatik, 89-90, 254.

s Hubschmann, Armenische Grammatik, 42-44.

- Hubschmam, Armenische Grammatik, 162 no. 291.
} 


\title{
DEMUTA, FOLK-HERO OF NISHEIGROM: MYTH AND SOGIAL STRUGTURE
}

\author{
BY
}

\author{
SCHUYLER JONES
}

I

Nuristan ('land of light') is the name that was given to Kafiristan ('land of infidels') by late 19th century Afghan conquerors. Nuristan is a region of high mountains and deep valleys on the southern slopes of the Hindu Kush in Afghanistan. Nisheigrom is one of nine villages in Waigal Valley, the valley itself being one of the five major valley regions of Nuristan. Together, the nine villages in this valley form a single political community, the members of which speak a common language-Waigali.

According to genealogies obtained in the field, ${ }^{1}$ Demuta lived in the village of Nisheigrom eighteen generations ago. ${ }^{2}$ The story of his birth and death is given here in translation from Waigali, together with a discussion intended to show why this particular story has persisted in this particular socicty and also to attempt to account for certain features which the story presents.

1 I carried oul anthropological fleldwork in Waigal Valley in the summer of 1966 , the winter of 1966-67, the summer of 1967, and again in the winter of 196768. My carlier fleldwork in Nuristan had been confined to the Ashkun-speaking peoples of western Nuristan (winter 1960-61) and the Bashgal Valley of NE Nurlstan (summer 1960, spring 1966, spring 1967). In 1967 I also spent a short time among the Kalasl Kafirs of the Rumbtur, Bomboret, and Berir Valleys of Chitral. This article was wrtten and accepted for publication in 1969.

I If we arbitrarily reckon a generation as twenty years and accept the genealogy as given, then Demuta lived some 360 years ago. My personal opinion is that he probably lived much earlier, partly because there is a tendency for genealogies to become compressed as unimportant links are forgotten, partly for reasons explained in the text. 


\section{The Birth of Demuta}

\section{II}

Pătūl had seven daughters. Six of them were married. One, the youngest daughter, still lived with her father. She was not married.

One day she went with Pătūl to the mountain pastures. When they came to a resting place on the mountainside, the girl told her father that she had to do something. She went into the bushes and $P \bar{a} t u ̄ l$ went on ahead. When he was gone, a large bird (called $p \bar{a} \bar{i})^{3}$ flew down. Its wing brushed the girl's shoulder.

After three months, the girl knew that she was pregnant. She went to her father and said, "Please find a husband for me." pātūl was angry. "You are not married," he said, "and yet you are with child. Why have you done this bad thing?"

Pătūl would not listen to his daughter's explanation. He sent her away.

When the influential elders (dïstii-diištii) of the village heard that Pätūl had sent his youngest daughter from his house, a few of them went to talk to him as mediators (dinwr̈ãi i).

"Why did you send your daughter away?" they asked. "You have gotten husbands for your other daughters. What has happened?" Pātūl told them that his daughter was with child.

The mediators went to see the girl to hear her story. She told them about the $p \bar{a} \bar{\jmath}$ and what had happened on the mountainside. When the mediators heard this they at once returned to tell Pătül. He heard them, but he refused to accept the story. Again, the mediators went to see the girl. "What shall we do?" they asked. "Your father does not believe the story."

"Wait until my son is born," she told them. "If he speaks before drinking milk, then you will know that my story is true. If my son cannot speak at once when he is born, then my father can do as he likes about me."

When the mediators told this to Patūl he said, "I do not want to see her in my house until this problem is settled."

Because Pătül would not let her live in his house, the girl had to go to the sawarl ama [segregated birth and menstration house]

\footnotetext{
s Prof. Georg Morgenstlerne, in his book on the Waigall Language (Norsk Tidsskrift for Sprogvidenskap, vol. 17, pp. 146-324, Oslo, 1954) translates päji as 'falcon, hawk, ktte', but I had thought from my informants that it was an eagle.
} 


\section{WAIGAL VALLEY, NURISTAN}

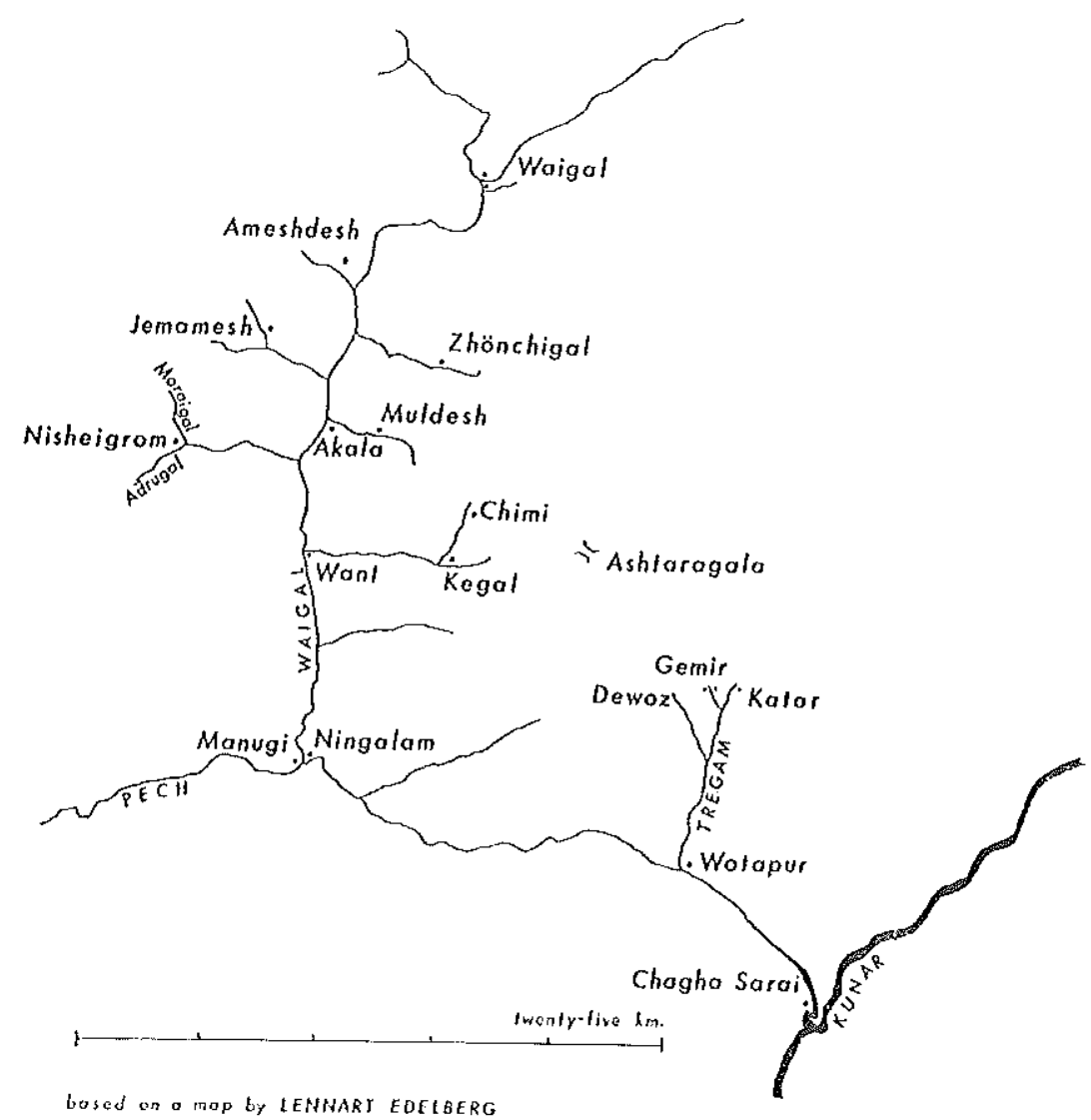

before the usual time. There she stayed until her son was born. As soon as the child was born, Pătull's daughter told the sawarti [woman in charge of the sawarl ama-a kind of midwife] to hurry and fetch the influential elders of the village.

The woman brought them and they stood at a distance while she went into the savart ama. "Take my son to them," said the girl. When the savarti carried the new-born boy out to show the elders, he spoke to them, saying, "Bring me a bow and arrows." One of the elders sent to his house for a bow and arrow. 
These were given to the infant. The boy took the bow and shot an arrow high into the air. It came down on top of digastīn [the great rock, representing the creator Imra, that dominates Nisheigrom].

At once the elders went to the house of Pătül to tell him what had taken place. "I am pleased that I was wrong," said Pătūl. "The boy's name will be Demuta."

When forty days had passed after the birth of her son, Pătūl's daughter could leave the šavar! ama. She took her son home to her father's house. There Demuta again asked for a bow and arrow. He took these and climbed to the top of diyastian. From there he shot an arrow high into the air. It fell in that part of the village called Demučem [also, sometimes Sunaratčem] where the houses of the lineage (māta) of Sunarat are today. Demuta asked the people to build a house for him there where the arrow fell, ${ }^{4}$

Demuta was a poor boy, because he had no father. His grandfather (Pātŭl) gave him some goats. Demuta worked very hard. He became a rich man. He killed many men and he gave many feasts. He was famous all over the Kalashum.

Demuta had one son. His name was Ăstān. From that Āstān all the people of the clan of Āstān are descended.

\section{III}

I first heard this story when I asked an informant to describe the genealogical relationship between the two clans of the village. Later, over a period of months, I heard it several times from different informants in Nisheigrom. In each case the story was not only substantially the same, but there was remarkable agreement in regard to details. The story as given here was tape recorded when Nurulla Khan of the Kusugdari, a descendant of Demuta, told me the story. Later, with the help of Persian speak-

\footnotetext{
- The house is sthll there. It is called Demuta kõt or Kantar kôt. It is said to be the oldest house in Nisheigrom. I believe that, in Kafr times, it served as a kind of clan shrine, the splrit or delly of the clan of Astân being A yan pränäa. This house was probably identical in function to the clan houses of Parun discusscd by Lemart Edelberg in A Kafr Goddess (Arts Aslatiques, vol. XVIII, Paris, 1968).
} 
ing informants from Nishcigrom, including Nurulla, I was able to complete my translation of the tale in Kabul,

In the course of studying the social, economic, political, or religious institutions of a society the investigator may find himself on occasion listening to long, repetitious, and sometimes tiresome stories of days gone by. No matter how astounding the details of such stories may be, they are always presented by informants as solemn fact. Unless one is particularly interested in mythology these tales may come as unwelcome interuptions in the middle of an otherwise satisfactory interview about inheritance, land tenure, or kinship terminology. As more is learned about the society, however, these stories often take on a decper significance. They no longer appear as a kind of entertainment that has evolved in the absence of literature, or the form which history has taken in a pre-literate society. Most of them, like the stories we have inherited from an earlier age in Europe, contain morals and the student of society can learn much about societal values from them. It is, however, in the realm of social relations that many myths serve their purpose in a society and are therefore of particular interest to the social anthropologist. It is commonplace to point out that myths are remembered and told and re-told down through the generations, not for their entertainment value, but because they explain something that needs explaining and cannot otherwise be explained. The story itself is a vehicle for an idea or moral and implicitly indicates correct behaviour and attitudes, thus reflecting cultural values.

In many cases the original meaning and purpose of European folk stories has been lost-only the stories remain. We may speculate on moral themes but in the absence of contemporary comments it remains speculation. In pre-literate societies we have the opportunity not only to record folk stories uninfluenced by written forms, but also the opportunity to record the comments of those who relate the stories. I suggest that the comments are more important than the stories themselves. If the story has socio-political significance then it would be well to attempt to discover that significance in the field, just as we investigate other social phenomena. The story itself is relatively unimportant, being merely an interesting and attractive package for a social 
theme. Unfortunately it is difficult if not impossible to distinguish what is wrapping and what is content without a thorough understanding of the society which gave birth to the story. After all, we are dealing with another culture; aspects of a folk story which attract our interest and excite our curiosity may be considered irrelevant by those who tell the story.

The story of Demuta, 'the man who had no father', is a story that has socio-political significance in Nisheigrom. By itself it is an interesting example of a folktale from an isolated and remote region in Afghanistan, but to understand the story's function in its indigenous setting and therefore the significance of the story to those who tell it, we need to know something of the social structure of the community where the story was recorded.

There is much in the literature of social anthropology to show that in some societies myths exist in order to account for relationships that are not accounted for in the genealogies. Genealogical knowledge is often not only insufficient, but is frequently in the hands of a few specialists such as chiefs, headmen, members of a royal family, or royal advisors and the biological realities of descent can then more easily be manipulated to serve sociopolitical ends. On evidence which is presented here I suggest that the same phenomenon-myth creation-also occurs where genealogical knowledge is extensive both with regard to generational depth and with regard to the number of people possessing such knowledge. In such cases the myth is necessary because the genealogical reality known to so many either does not agree with social reality or it does not agree with some desired social reality. How the myth comes into being and who creates it is quite another question. The important point is that the myth has a function in the society, hence its existence. ${ }^{5}$ When it was created and who created it are irrelevant considerations, though it would be interesting to observe and record the process. In the case of the myth which is presented here, it may have already existed from an earlier period and the hero of it was subsequently given a place in the genealogies to serve a particular purpose.

A factually correct genealogy is two things at once: a record

s Cf. Bronislaw Malinowski. Myth in Primitive Psychology, in Magic, Science and Religion. 1926. 
of biological descent and a charter granting rights and obligations to a specific group of people. The first is biological, the second is social. Since the latter is more important than the former, the accuracy of the record of biological descent may be sacrificed in the interests of socio-political ends. It is often extremely diffcult to investigate this process or even to perceive that it has taken place. In view of this it is more useful at the ontset to regard genealogies as social phenomena rather than biological history. In any case, it is in the social realm that they are significant in every day aflairs. ${ }^{8}$

The population of Nisheigrom, like the other villages of Waigal Valley, is divided into social classes. The socially, politically, and numerically dominant class, atrožan, is divided into clans and lineages. Skilled and unskilled craftsmen and workers (bari and setvala) make up the socially disadvantaged classes; they are distinguished from the politically significant atrožan by their modes of livelihood and by numerous negative social sanctions which effectively maintain the status quo.

In Nisheigrom all atrožan belong either to the clan of Pātūl (Pätüldari) of the clan of Āstān (Āständari). Descent is traced through the male line and a man of Nisheigrom can reckon his exact genealogical relationship not only in respect to every man in his lineage, but every man, woman, and child in his entire clan. Elders can recite genealogies by the hour. As far as I have been able to determine, none of the few literate men in Waigal Valley have actually written their genealogies down except for those which I encouraged a student to record. ${ }^{7}$ In Waigal Valley it is not uncommon for a man to be able to name twenty generations of his ancestor's, though none of my informants there could seriously rival those elders in the Bashgal Valley who provided me with the names of up to 45 generations of their ancestors. ${ }^{8}$

- Sce $A$ Genealogical Charter by Laura Bohamman, Africa, vol. 22, No. 4, 1952

- In Kamdesh and Baragamatal in the Bashgal Valley I encountered a few men who, on their own initiative, had written their genealogies, but it is still very
much the exception in Nuristan.

${ }^{8}$ Cf. Jones, Schuyler. The Political Organization of the Kam Kafirs. Kgl. Dan, Vid. Selsk. Hist. Filos. Medd, vol, 42, No. 2, 1967. Copenhagen. In 1929 Georg Morgenstierne obtained a genealogy of 54 generations from an old IKafir in Chitral. Cf. AEtlctradisjon hos Kafirene $i$ Hindukusj. Maal og Mimme, Osto, 1950, pp. 155-162. 
These genealogies are not mercly lists of agnates, they ramify widely and I have spent entire aftermoons recording the various branches of elan and lineage from a single informant.

Approximately half the alrožan of Nisheigrom belong to the clan of Āstān-those who trace descent from Āstăn-while the other half belong to the clan of Pãtul by virtue of the fact that they trace descent from Pātūl. Since Nuristani villages are extremely compact, physically isolated from each other, and traditionally politically autonomous, it is not only desirable but essential that a high degree of social solidarity exist within the village. The political danger is that a dispute between members of one clan and those of another could divide the village into two opposing camps-one clan against the other. One way of forging a bond between the two clans is to show a genealogical relation. ship between Pātül and Āstăn and appeal to the principle that kinsmen should not fight with one another. I suggest that in a society where genealogical knowledge is less extensive the problem might be solved by general acceptance of the fiction that Pătül and Āstān were brothers. No one would be able to contradict the story because the gencalogical evidence would have been forgotten. In Nisheigrom, however, there are many men who can trace descent from the founders of the village down through pātūl or Āstann to their own particular lineage segment. Such knowledge prevents acceptance of the obvious solution to the problem of village solidarity. Everyone knows that Pătul and Āstăn were not brothers. The myth must take a different form. The form it takes in this case is that while the hero's mother is known, his father is not. With Demuta's father out of the way several socio-political ends are attained:

(i) the genealogical connection between the clan of Pătūl and the clan of Ästann is established without fear of contradiction (not knowing the father, descent can only be traced through the mother),

(ii) the virtue of Pătūl's daughter is preserved, as the story makes clear,

(iii) genealogical expertise is not offended. 


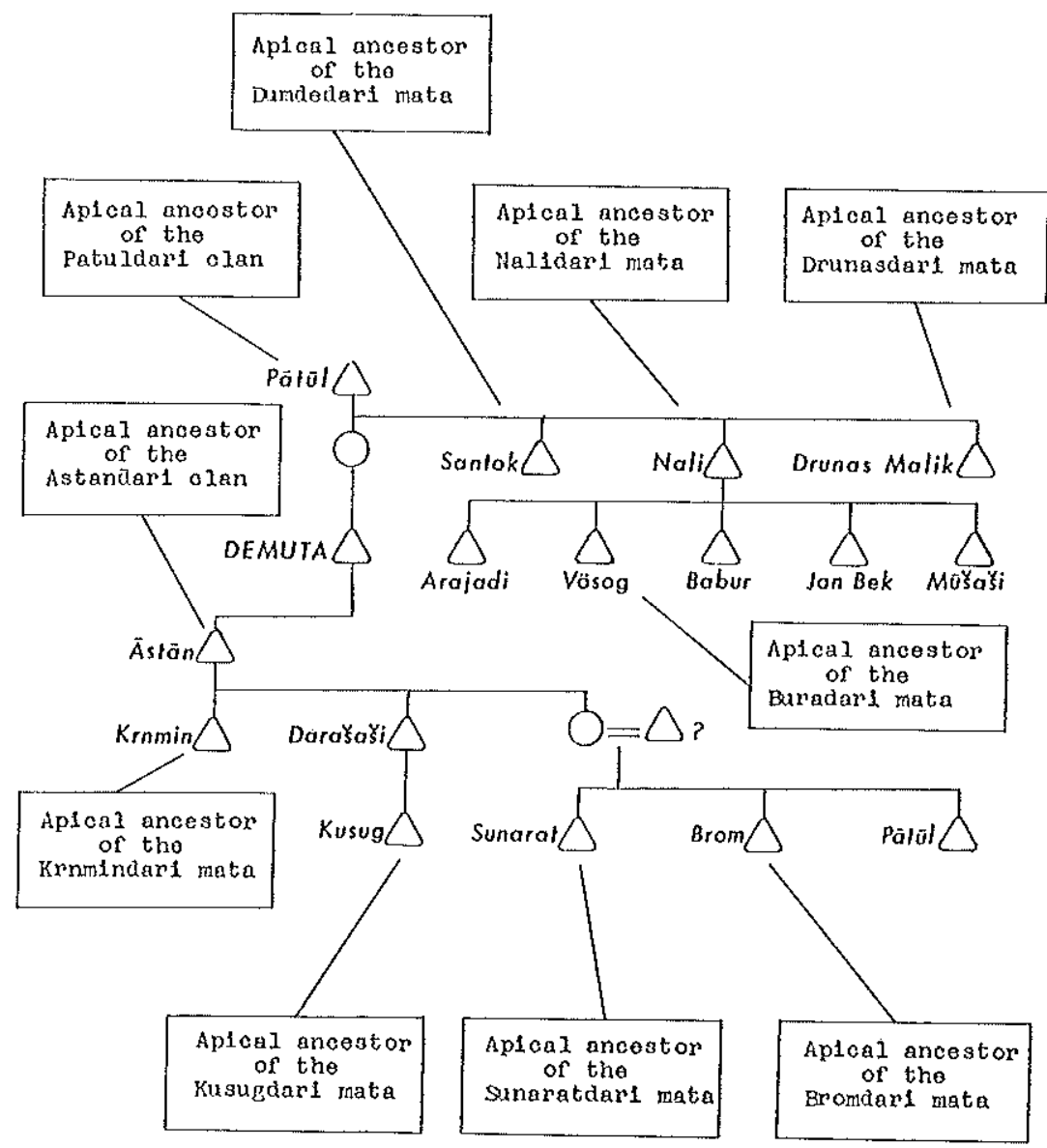

mata $=$ lineage

IV

Clans and lineages usually derive their names from the apical ancestor, but there are exceptions: Santol is the apical ancestor of the Dumdedari lineage but the lineage gets its name from the fact that Santok's wife was from Dumgal. There is a long story that tells how Santok came to get a wife from the Afghan Safis of Dumgal. The Buradari lineage is so named because the founder, Vøsog, was bur-a deaf-mute.

Although descent reckoning in Nuristan is patrilineal, use is 
occasionally made of female links. We have noted that there is a female link between the two clans of Nisheigrom. Furthermore the lineages ( $m \bar{a} t a$ ) of Brom and Sunarat are grafted onto the clan of Astann by the genealogical knowledge that Āstān's daughter had three sons: Pātūl, Brom, and Sunarat. The name and lineage of Āstān's daughter's husband is not known and therefore is not important. 'The point is that Astann is the grandfather of Brom and Sunarat-from this they and their descendants derive their social identity. It is known that both lineages originally belonged to the clan of Patul and that the lineage of Sunarat was adopted (eken-loy) by the clan of Astann. At the same time, the lineage of Drunas was also adopted, though in that case there has been no corresponding adjustment of the genealogies to accommodate the new social fact.

The adoption of the two lineages came about in this way: Nisheigrom is located at the confluence of two streams which drain the valleys of Moraigal and Adrugal. The people of the clan of Pătül own all the land in Moraigal; the people of the clan of Astan own all the land in Adrugal. This arrangement caused problems, as one informant explained: "The Āstanndari had many fields, but few people. The Pantūldari had many people, but little arable land. The elders solved the problem in this way: the Pătūldari agreed to give two lineages to the Āstăndari. They gave the lineages of Sumarat and Drunas. These two lineages joined the clan of Āstān. 'The problem was solved."

\section{$\mathrm{V}$}

I suggest that there is some ground for considering the story of Demuta, as Pătūl's grandson and Āstān's father, to be a fabrication. It may well be that there was an ancient folk-hero of Nisheigrom named Demuta who did remarkable things and lived so long ago that he had no place in the genealogies. It subsequently became convenient to place him in the genealogies as a link between Pătāl and Astān for the reasons given above. My reason for suggesting this is not complicated. If descent had been traced from Demuta all along, one would expect a descent group bearing his name to have evolved. Yet the clan is named 
after Demuta's son (Āstān). This in itself is an anomaly, particularly as the son was in no way as distinguished as the father. As it is, it seems that the clan name Āstann had already become established long before it became useful to regard Demuta as Ästān's father. This was only possible if Āstān's father had been forgotten.

In Nuristan descent is normally traced through the male line, but use is made of female links whenever it is expedient to do so. Acceptance of the female link in this case means that every atrožan in Nisheigrom can trace descent from Pãtūl. In this light, the story of Demuta is a kind of mandate for maintaining social solidarity in this village.

\section{The Death of Demuta}

Demuta had a soli from Gemir. ${ }^{9}$ Demuta had made solibar [brotherhood] with this man because he had asked it. Demuta's soli had no son. He went to the seer (wřễ̌) to talk to him about not having a son. The seer told him, "You have a famous soli. Let him sleep with your wife. Then you will have a son." The man went home and thought about it. Then talked to his wife, telling her what the seer had suggested. He told his wife to do it.

When Demuta next came to his soli's house in Gemir, the man greeted him and said that he had to go away for a day or two, but Demuta should stay in his house while he was gone. Demuta agreed and when the husband had left, the wife tried to get Demuta to do what the seer had suggested. Demuta refused, saying, "My soli is like my brother. Thercfore you are like my sister. I cannot do what you ask."

Two days later the husband returned to Gemir and at once asked his wife what had happened. She told him that nothing had happened; Demuta had refused.

\footnotetext{
- Two men may become 'brothers' by participating in a ceremony in which a goat is sacriflced and the kidneys are removed and cooked. A witness then formally announces to the men that "as these kidneys are a pair, so you will be a pair throughout your lives". One man then eats one kilney and his friend eats the other. Solibar establishes a relationship identical to that between men who have the same father, though blological brothers call each other brä.
} 
The man was suddenly afraid that Demuta might return to Nisheigrom and tell someone what his wife had asked him to do. Demuta's soli watehed his chance and put poison in Demuta's food. When he had eaten, Demuta began to feel ill and decided to go home. He set out at once from Gemir, crossed the Aštaragala Pass and started down into the Waigal Valley. When he reached Chimi he was so ill that he could not walk further. 'The men of Chimi carried him to the edge of Nishei territory where they met people from Nisheigrom who took Demuta from there.

On the trail they suddenly found a huge boulder blocking the way. They could not carry Demuta by this place because the mountainside was very steep. Demuta told them, "Put me down and give me my waš $l i k .^{10}$ He could not stand, so, lying on the ground, he struck the boulder and it broke so that they could pass. ${ }^{11}$

Demuta was tired. He told his friends, "Let us rest." From his resting place on the ground, Demuta saw bees round a honey tree. He asked a man to make a fire and prepare a bowl of warm honey for him. When he had eaten this, Demuta became quite well and walked on to Nisheigrom without help.

As he walked, he thought about what had happened. By the time he reached Nisheigrom, Demuta was angry with his soli. The next day he made preparations and set out for Gemir to kill the man who had poisoned him.

Reaching Gemir, Demuta went directly to his soli's house and shot an arrow through the open door. From inside, the soli shouted, "Forgive me. I will do whatever you ask, but don't kill me."

The soli's wife snatched the big iron griddle (kiiliis) from the tripod at the freplace and held it in front of her husband like a shield. Outside, Demuta said nothing. He shot another arrow into the house.

With his wife protecting him with the griddle, the man reached

\footnotetext{
10 was'lik is a special ceremonial axe-not used for cutting wood-but carried like a swagger stick by all men of high rank in Kaflr times. In Nisheigrom I was told that Demuta's was'lik still existed, but $I$ did not see it. One member of Demuta's family said that a foreigner had taken it; others disagreed.

11 'Today this place is called Demuta was'lik vina.
} 
back to get his bow and arrows. Then he shot an arrow and Demuta fell before the door and died there.

The people of Gemir were afraid. For a long time they did not go near the body and Demuta lay where he had fallen. After a time his body began to swell up. It became very big. Finally one of the men of Gemir approached the body and struck it with an axe. As soon as he had done this there came from Demuta's body thousands of locusts. They spread out over the village and ate everything. It was nearly harvest time. The locusts destroyed all of the crops and there was famine in the village. Never had the people of Gemir seen such a plague of locusts.

When the men of Nisheigrom heard that Demuta was dead they went to Gemir and killed the people; they destroyed the village and took away the goats and cattle. Later, when those men of Gemir who had fled to the mountains returned, they found their village in ruins. "We must sacrifice a cow for Demuta," they said,

Every year they sacrifice a cow for Demuta just before harvest time. If they do not do it, the locusts come and destroy the crops. They still do it today. If you go to Gemir before harvest you will see them sacrifice a cow for Demuta. ${ }^{12}$

\section{BIBLIOGRAPHY}

Bohannan, Laura. A Genealogical Charler. Africa, vol, 22, No. 4.

Edelberg, Lennart. Nuristanske Solvpokaler. With a 1:250,000 map of Nuristan. KUML (Yearbook for the Jutland Archaeological Society, Aarhus, Denmark) pp. 153-201. 1965.

Edelberg, Lennart. A. Kafir Goddess. Arts Asiatiques, vol, XVIII, Paris, 1968.

Edelberg, Lennart. Some Paruni Myths and Hymns. Acta Orientalia, vol. XXXIV; Copenhagen 1972.

Jones, Schuyler. An Annolated Bibliography of Nuristan (Kafiristan) and the Kalash Kafirs of Chitral, Part One. With a Map by Lennart Edelberg. Hist. Filos. Medd. Dan. Vid. Selsk. vol, 41, No. 3, Copenhagen, 1966.

\footnotetext{
12 Since the signifleance of Demuta's story is sociological rather than historical It is perhaps irrelevant to add that the people of Gemir appear never to lave heard of Demuta and deny that they sacrifice a cow before harvest.
} 
Jones, Sehuyler. The Political Organization of the Kam Kafirs: A Preliminary Analysis. Hist. Filos. Medd. Dan. Vid. Selsk, vol, 42, No. 2, Copenhagen, 1967.

Jones, Schuyler. A Bibliography of Nuristan (Kafiristan) and the Kalash Kafirs of Chitral, Part Two, Selected Documents from the Secret and Political Records, 1885-1900. Hist. Filos. Medd. Dan. Vid. Selsk. vol. 43, No. 1. Copenhagen, 1969.

Malinowski, Bronislaw. Myth in Primitive Psychology, in Magic, Science, and Religion (1926).

Morgenstierne, Georg. AEttetradisjon hos Kafirene i Hindukusj. Maal og Mime. pp. 155-162, Oslo, 1950.

Morgenstierne, Georg. Some Kati Mylhs and Hymns. Acta Orientalia vol. 21, pt. 3, pp. 161-189, Copenhagen, 1951.

Morgenstieme, Georg. The Waigali Language. Norsk Tidsskrift for Sprogvidenskap, vol. XVII, pp. 146-324, Oslo, 1954. 


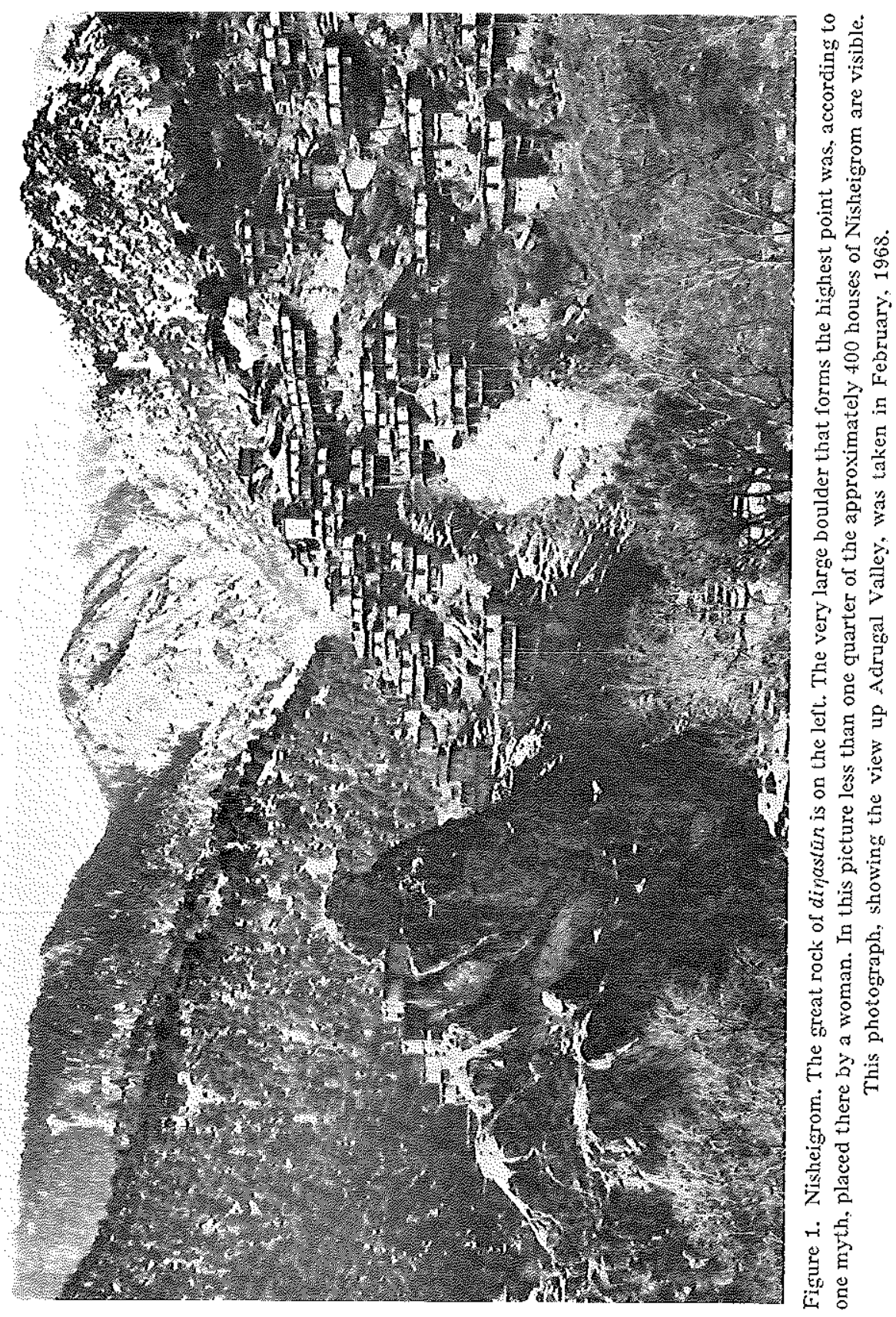




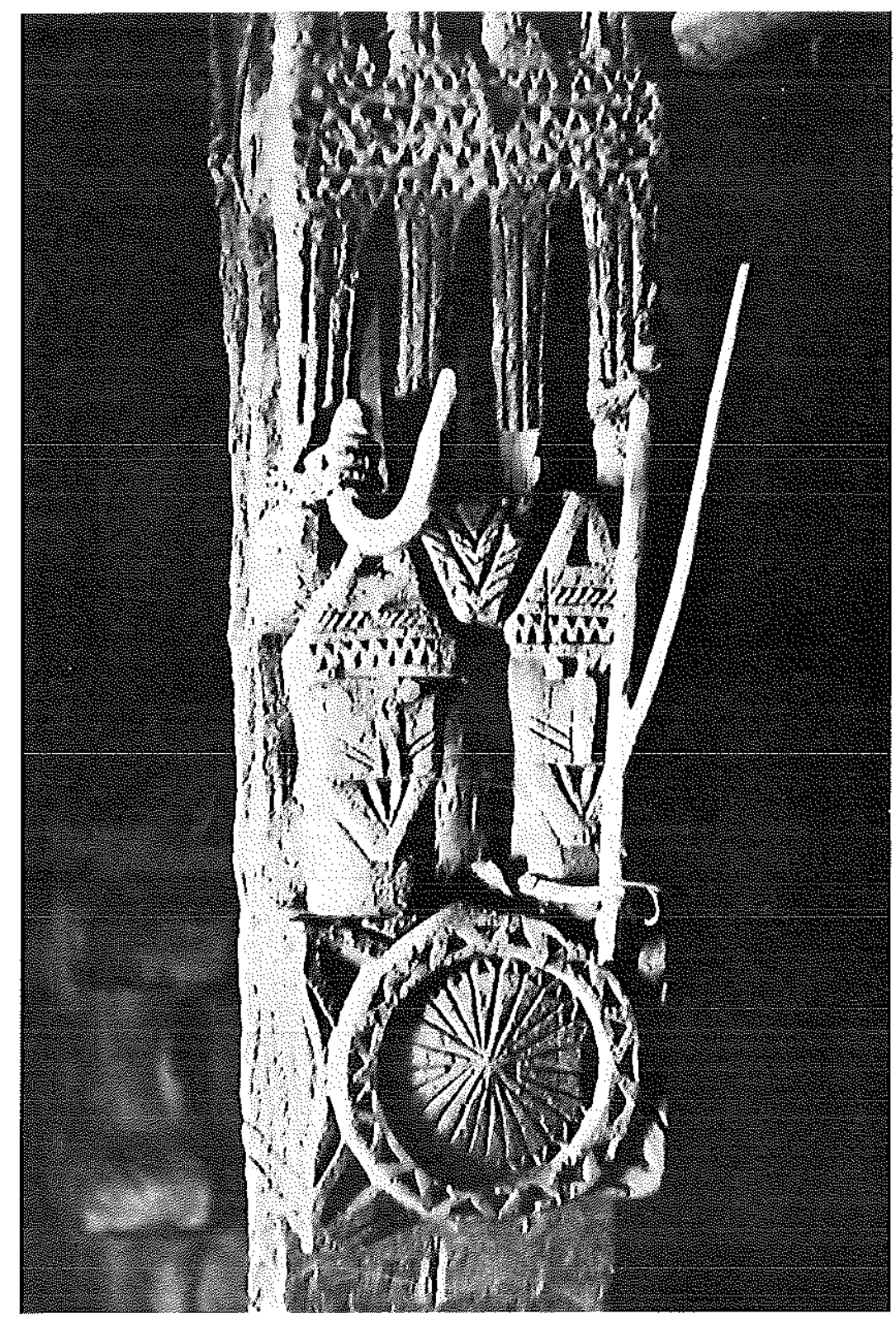

Figure 2. Section of one of the four columns in the main room (ama) of Demuta's house in Nisheiglom showing human faces and, below, symbols of rank. Across the face on the right and above that on the left are wonden hooks for clothing. 'The face on the left has been partitly destroyed by knife or axe-possibly an expression of Muslim dislike for anything resembling an idol. Photograph Laken in Mareh, 1967. 


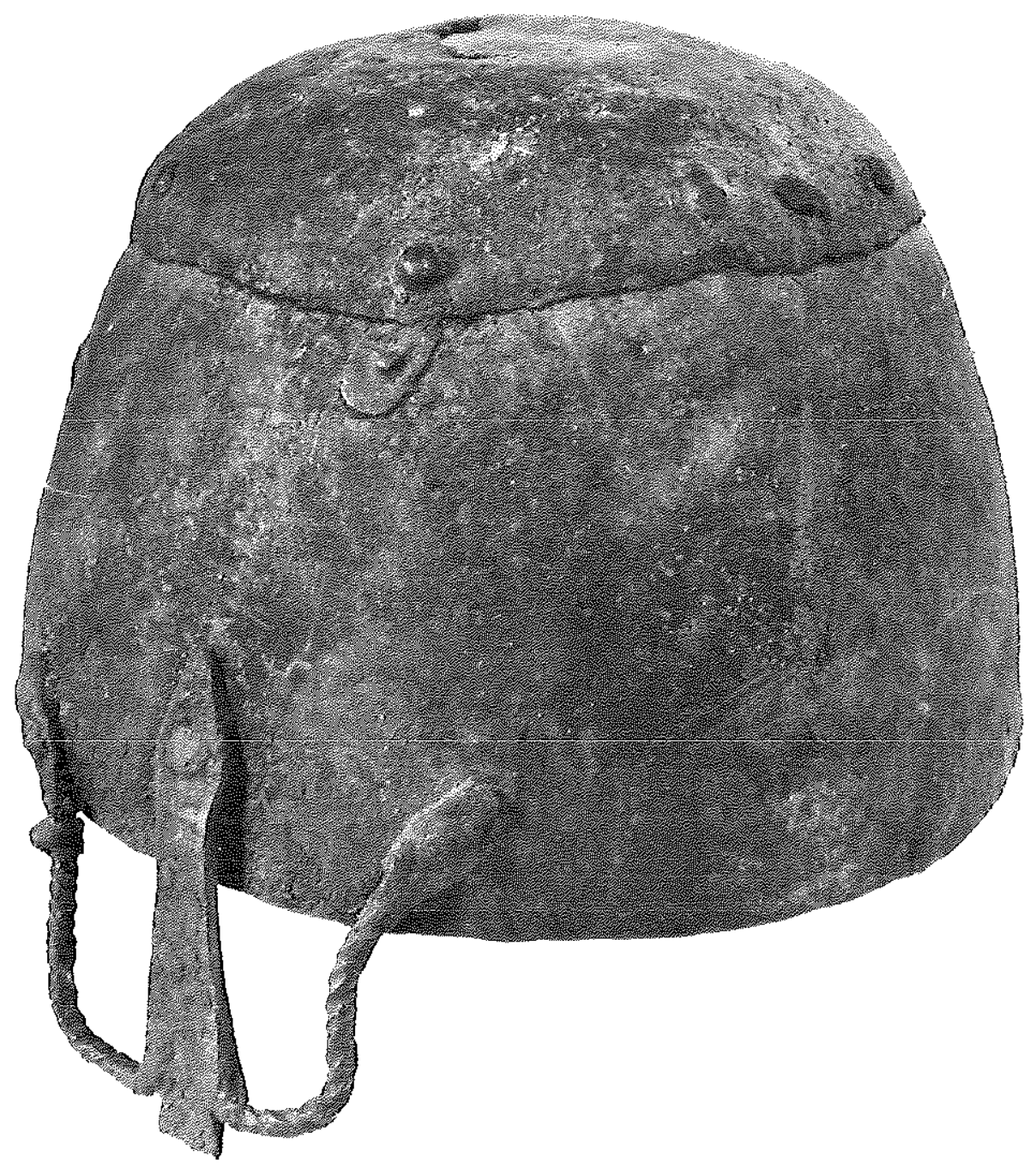

Figure 3. 'Demuta's helmet', pictured above, was found hanging at the top of a column in his house in Nisheigrom. Informants referred to the helmet as milcou sei tapi, 'fighting head hat'. Demuta's descendants atlached Jitte importance to the helmet and it was purchased from the present owmer of the house. It is now on loan with the Kafir collection at Moesgaard pr. Hojbjerg, Denmark. 


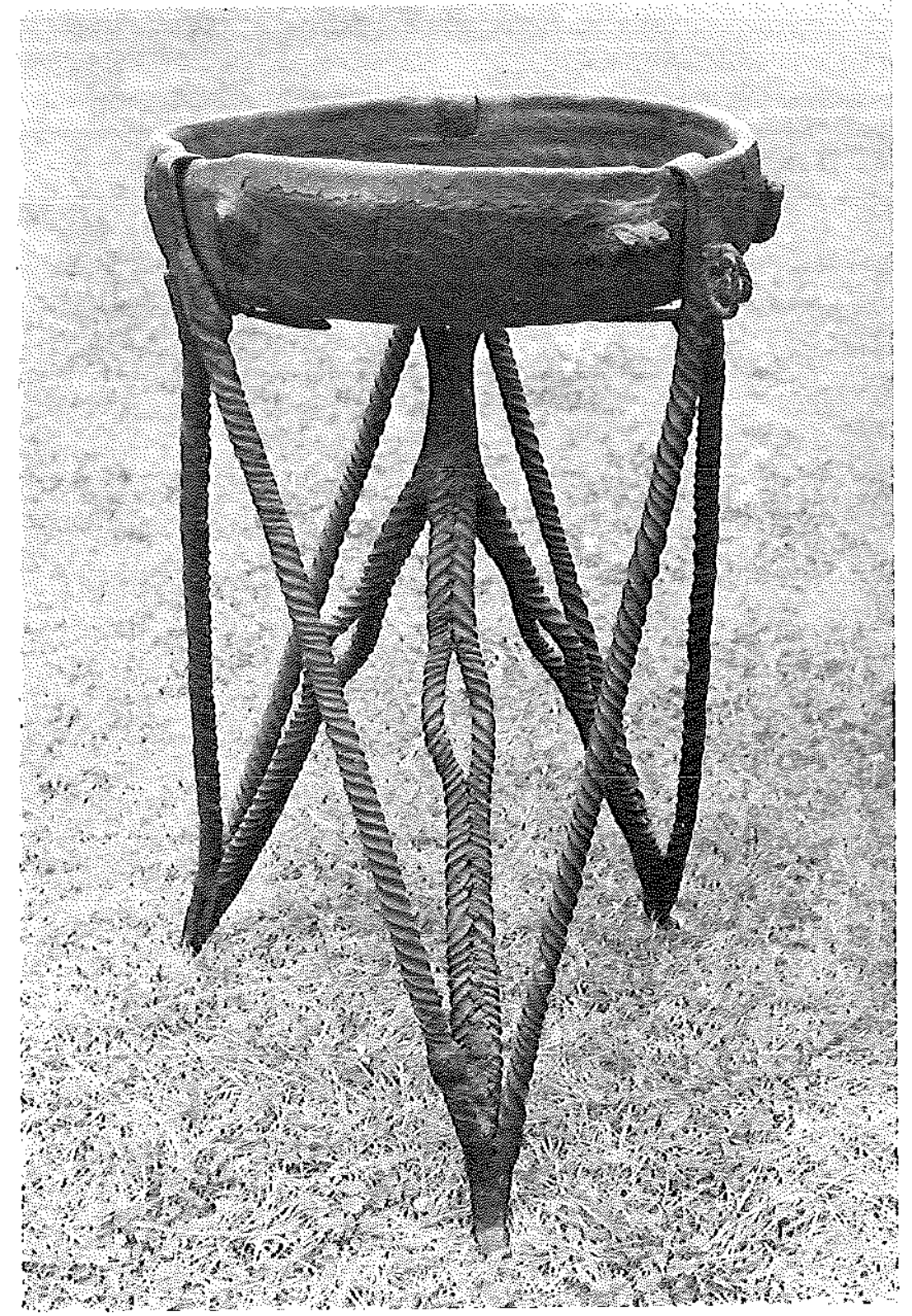

Figure 4, 'Pātül's bäspe', an example of an early type of Waigali lable which gets its name from the fact that the wooden bowl is supported by 12 heavy rods of twisted wrought iron (bäs, 12). This particular $b \bar{a} s \bar{p} p$, said to have belonged to Pätal, is the only known example of an early type characterized by a wooden bowl with rounded sides fecorated with the carved heads of bulls, supported by very heavy iron legs. (Height: $54 \mathrm{~cm}$; diameter of bowl: $30 \mathrm{~cm}$.) Compare with the more common type of bāspe pictured on p. 187 of Lennart Edelberg's article on Nuristanske Solppokaler (KUML, 1965). 


\section{SOME PARUNI MYTHS AND HYMNS ${ }^{1}$}

BY

\section{LENNART EDELBERG}

\section{Introduction.}

From 1947-49 I was a botanical specialist with the 3rd Danish Expedition to Central Asia under the leadership of the Danish explorer Henning Haslund-Christensen. In the course of that expedition in Afghanistan and later, on the Danish Scientific Mission to Afghanistan 1953-54, and in 1964, I visited the remote Parun Valley in Nuristan (Kafiristan) on several occasions.

The myths and hymns published here were recorded then, but under variable and-from a scientific point of view-mostly unsatisfactory conditions. One condition was invariably regrettable: I am neither a phonetician nor a linguist.

The ideal thing would of course have been to have had a tape-recording, a transcription in phonetics, a word-for-word translation, and a free rendering of the text and its meaning in some common language. We are hardly ever so fortunate. However, these texts may show that remmants from the pagan (Kafir) time do still exist, and thus may induce some scholar to go there.

The Parun Valley (Robertson 1896: Presungul) is situated between the eastern and western Kati area. Its geographical features are described in the text to my map, where most of the holy places I was shown are indicated. The small population of the Parun Valley belongs to the so-called Safed Posh, the whiteclad, contrary to the Siah Posh, the black-clad Kates, but it

1 Morgenstierne 1949 has published a single Paruni hymı to the goddess Sənjẵa (informant: W'odl from Pronz, whom I met in 1953 and 1954; Morg. 1949 p. 308, VI) and 1951: Some Kati Myths and Hymns. In 1967 Morgenstierne furthermore published: Some Folk-Songs from Nuristan, and in 1968: Mylhological Texts from the Kates of Nuristan. My own article was accepted for publication in 1969.

3 Acta Orientalla, $\mathrm{x} \times \mathrm{XIV}$ 
would carry us too far to give an ethnographical description of the Parun tribe here.

Sir George Scott Robertson says in his outstanding book about The Káfirs of the Hindu-Kush that "In Presungul there is a distinct atmosphere of religion." (p. 379), and that "The purest form of the Kálir religion is probably to be found in the Presungul." (p. 379). Furthermore that "In Presungul every village is supposed to be under the care of one special god ..." (p. 377), and that "'The chief temple to Imrá is at Presungul, at Kstigigrom," which is undoubtedly the most sacred village in the whole of Káfiristán." (p. 389).

This atmosphere of religion is still felt, but I cannot define it. I think, however, it must be the background for the mulla of Kushteki being honoured as the chief mulla of all Nuristan.

It is therefore rather strange that the other Nuristani definitely do not look up to the Paruni. One of my informants in Kushtos thus voiced the opinion that "The Paruni are sheep! They sleep on their stomachs!"

This, however, is in accord with Rob. 1896 p. 161: "In the beginning of the world God created a race of devils. He soon afterwards regretted having done so, but felt Himself unable to destroy all those He had so recently endowed with breath. But Moni (sometimes called Muhammad by Káfirs, under the impression that prophet and Muhammad are synonymous terms), grieving at the terrible state of affairs, at length obtained a sword from Imrá, and was given permission to destroy all the devils. He killed very many, but seven, the ancestors of the Presuns of to-day, managed to escape him".3

Robertson writes (p. 396): "In Presungul the idol-houses are much more carved and ornamented than in the Bashgul Valley, while the god is often shown seated under a wedge-shaped roof, and sometimes engaged in playing a musical instrument ..."

2 Kushteki (Kushtegi).

3 One must invariably ask: Is there any connection between these seven devils and the seven villages of Parun? And posstbly the seven bad sons of Purdi (Myth no. $6 \mathrm{~A} \& \mathrm{~B}$ ), who lived in Pronz, and the Purron, seven golden brothers, mentioned by Elphinstone 1815 p. 621 . 
"Occasionally the shrine is placed on the top of a village tower in Presungul, a plan I have seen in no other district."

And about Dizane's [Disni's] temple at Kamdesh in the Bashgul Valley, Robertson writes: "It was built by men brought from Presungul for the purpose. It is covered with carving, and has the wedge-shaped roof so common in Presungul, ... Along both sides of the base of the sloping roof poles are fixed, and support wooden images of birds, said to be pigeons. This is really a very pretty little temple." (fig. 1).

Apparently the Paruni had their shrines well-kept in 1891 and when 1 arrived in Parm in 1948 the village towers were still in good repair, many of them with four long horns of the wild markhor on poles on their roofs. In 1964 only the village tower of Shtiwe was in good repair (fig. 2).

The Kafirs were known as quarrelsome, but the Paruni maintain that they never fought with each other. In fact the Parun Valley is one community, and many religious feast were common for all the valley.

The reader will soon discover that it will be valuable to have Robertson 1896 (especially p. 376-411), Morgenstieme 1951, Buddruss $19600^{4}$ and Snoy $1962^{5}$ to hand when studying the myths and hymns recorded here.

The numbers of the tape-recordings refer to one catalogue comprising 1947-49 \& 1953-54 made by Thomas Alvad and another comprising 1964-recordings (both manuscripts in the library of the Danish Folklore Archives, Copenhagen, where the tapes are also to be found).

I want to express my sincere thanks to Klaus Ferdinand, Moesgård, and Ahmad Ali Motamedi, General Director of the Antiquities of Afghanistan, for generous permission to make use of their

4 Unfortunately Professor Georg Buddruss has not yet published his book: Die Sprache der Prasun-Kafiren, which is founded on his fleld-work in the Parun Valley in the spring of 1956.-But "while the grass grows, the steed starves!" As the same argument can be used against me, I publish now my modest material, which was mostly collected in 1948-54.

5 Snoy is rendering many of the Parun myths collected by Professor Buddruss in 1956. It is much to be hoped that the texts themselves will be published, so
that a comparative study can be made. 
diaries from our common wonderful journey in Nuristan 1953 , to Professor Georg Morgenstierne, Professor Georg Buddruss, ${ }^{6}$ Professor, dr. phil. Jes Asmussen, Faridun Vahman, and to Schuyler Jones for valuable help and good advice, to Babamorad Feraghi, of the Kabul Museum, and to Aage Andersen for their excellent map, and to Jill Glenstrup, Jytte Thomsen, Lis Gramstrup, and UIf Timmermann (U. T.) for their help with the manuscript.

Last, but not least, I want to thank the Carlslerg Foundation which has always generously supported my investigations.

\section{Mylhological Map of Parun.}

Cf. fig. 34.

The Parun Valley was formed during the Ice Age by glaciation; probably a glacier from the Ichemgol and another from the Wüäsgol (Wezgal). 'This glacier scems to have gone as far as the ravine a little below Pashki. The valley to that point is therefore U-shaped, while the valley further down, including its continuation in the Pech valley, is V-shaped.

Avalanches from the sides of the valley will sometimes even reach the river bed here and there, and when this happens the snow lies so deep that it does not melt until mid-summer.

The slopes at Pashki (altitude of Pashki 2,500 metres) are covered by coniferous forests, but these do not reach further north than Dewa, and at Shtiwe (altitude 2,800 metres) the slopes are at the most covered by thickets here and there.

The base of the slopes on the left bank of the river at Pashki are covered by groves of hazel-trees (Corylus colurna). In the bottom of the valley we find the cultivated fields: slightly sloping terraces irrigated from the small side-streams.

Along the river-side or at the side-streams near the villages there are rows of water-mills; small mills with a vertical axle and horizontal millstones. ${ }^{8}$

\footnotetext{
- Professor Georg Buddruss has most kindly read through my manuseript and made valuable corrections and suggestions, which I have marked 'G.B.'.

7 gal, gol, gul means valley.

8 Lennart Edelberg 1960 b.
} 
The spring ploughing takes place in $\mathrm{May},{ }^{9}$ and the threshing is finished in the last week of October, when the flocks and herds will be brought from mountain pastures to the villages.

The seven villages are marked on the list with Roman figures, no. I (Pashki) being the lowest. No. VII (Düröshwa) was deserted even in Kafir times.

Pashki and Zumu are built on the slopes; Kushteki at the foot of an almost vertical eliff; Dewa, Pronz, and Shtiwe in the middle of the valley. The last three villages form rcal têpes (fig. 3). 'The narrow lanes between the two-storied houses have been filled up with rubbish so that the houses to-day are chiefly unclerground and their rooms, therefore, very smoky. Archaeological diggings in the narrow lanes of these villages would no doubt give interesting and extraordinary results. The têpes are presumably not so very old, and the finds may have a direct bearing on the evidence of oral traditions,

Pashki, Zumu, Dewa, Pronz, and Shtiwe had one or more towers each in 1948 when I first stayed in the valley. In 1964 only Shtive had a well-kept tower.

On the map 8 means "tower", 垈 "mosque", and $\square$ Kafir temple or other holy building or sacred stone.

Ajetokwö beneath the peak of Azei:

Robertson 1896 P. 65 (and 399): "The point of junction [of the Parun and Kantiwo rivers] is a very sacred place in the Káfir imagination. On the narrow tongue of land, which separates the rivers jusl before they mingle, there is a rocky ridge, where the gods were wont to assemble, and a peculiar sacred stone, placed there by Imrá."

I. Pashki (Paruni: Uș'iiil); 100 houses (fig. 4):

1. Disni-temple (fig. 5).

2. Moni-temple.

3. Two towers comnected by walls (Ahmad Ali Motamedi, diary 1953 p. 201 : Ram-yu).

4. Blacksmith's part of the village.

\footnotetext{
Ahmad Ali Motamedi \& Lemart Edelberg 1968 and Lemnart Edelberg 1968.
} 
5. Goal-pen 1948 belonging to the blacksmith Amir Shah. According to Myth no. 1 the house of the ancestors of the blacksmiths moved from Shtive to this place (cf. fig. 24).

6. Stone on which salt is given to the flocks and herds.

7. Platform.

Opposite the village the valley of Bar-Gal (barigal? = the valley of craftsmen) opens into the Parun Valley. At the right side of the upper end of Bar-Gal the mountain pastures of the blacksmiths are located (cf. Myth no. 2).

In the neighbourhood of Pashki a rock rises from the river bed. ${ }^{10}$ Its name is Manis.. ${ }^{11}$ Those Kafirs who claimed to have killed enemies during the summer season without having brought the heads of their victims to their own valley, went to this cliff. If they passed it safely, they were trusted. If they had told a lie, they would fall into the river.

Those who had passed the test were honoured by the prominant people and went up through the valley to all the villages and thus got a distinguished reputation among their people.

II. Zumu ${ }^{12}$ (Paruni: $\left.Z u m{ }^{\prime} \bar{u}\right) ; 16$ houses:

The bridge of Zumu does not lead to the village itself, but to the winter-stables that are situated near the river-bed. The bridge is furnished with a remarkable gate (fig. 6), which can be locked.

III. Kushleki (Paruni: Üs'äil; according to Barrington 1960: Imragān. Waigali: Yamraidesh); 24 houses (just after conversion 50) (fig. 7):

Rob. 1896 p. 389 : "Kstigigrom, which is undoubtedly the most sacred village in the whole of Káfristán"13:

10 The rock has not been shown to me. The informant was from Pronz.

11 My diary has: Manteš. G.B.: Man-t-iș is the localive case.

12 Map in Deutsche im Hindukusel 1937 has: Tusum. G.B.: tu-Zum is the locative case.

18 Klatus Ferdinand, diary 1953: If a quarrel has arisen between two villages 
1. The ruins of the chief temple to Imra (Rob. 1896 p. 389) (lig. 8). ${ }^{14}$

2. Stronghold above the village (fig. 9).

3. "Imrá's water" (Barrington 1960).

4. Wash-place, where the women assemble when washing.

5. Cemetery (Deulsche im Hindukusch, $1937 \mathrm{Abb}, 112$ and p. 256) (fig. 10).

6. Pit leading to Yurdesh, the nether world (Rob. 1896 p. 380 and 393). The spot, but not the actual hole, has been pointed out to me by the local mulla in $1964 .^{15}$

Opposite the village is seen the beautiful peak of Sïskömiint from which the sum is said to rise on the shortest day of the year (Edelberg, Lemnart, A. Schäfer, W. Lentz, 1959). Actually the sun seems to rise a little further towards the South on the day of winter-solstice.

IV. Dewa (Paruni: Ǘ' $\left.\dot{c}^{\prime} l\right) ; 58$ houses:

Rob. 1896 p. 396: "At Deogrom [Dewa] there is a Monitán (Moni ${ }^{16}$ place i.e., shrine) where the "prophet" is made of an extraordinary shape. $\mathrm{He}$ is furnished with large circular eyes with a dot in the middle; he has cat-like moustaches, and appears to be holding his head in his hands, the face peering out between the points of long horns, which, starting from below, cross and recross each other till they reach the god's chin."

Cf. furthermore Rob. 1896 p. 400: "two small patches of glacier ... called Moni's marks."

(in Parum), they go to Kushteki to have it settled. If this brings no result, they go to the Kusum-tadba in Shtiwe (probably to Zāmann cf. No. 11 A).

14 In this temple was a big god sitting on a horse (= KK 17, cf. Lennart Edelberg 1960a) and a small goddess (Paruni: banigya diz) sitting on a taboret. The god was Măra (Mareira) and the goddess Pusāsi or Disni (Dizeile). The goddess was placed at the left side of the god.

16 In Kafr time the hole had a stone cover. A horse from Badakshan was sacrificed here every year. The blood was poured into the hole by a man [probably a priest] who turned his face away. When the mullas removed the stone cover nothing was found or seen (informant: Zāmăn, Kusum-tadba, Shtiwe).

16 Morgenstierne 1949 p. 283: M'än-dĭ; (Kati: Mön). 
Same page: "an isolated fragment of gneiss" (at Dewa). This stone has connection with Moni, too.

Rob. 1896 p. 410: "Nong and Shomde" are deities peculiar to the Presungul. 'The latter is the tutelary deity of the village of Diogrom."

In the middle of the village (fig. 12) is a house of assembly called amal (fig. 13), a square-roofed building with a central pillar and benches along the four sides. The room is walled to the north, west and south and open to the east; at the opening a flat stone has been placed on which the speaker stands (fig. 14).

Outside the amal, to the eastern side, is an open place of the same size as the amal ( $4 \times 4$ metres). This place is called uštimsagam.

At the north-eastern corner of the ušïm-sagam is a house belonging to a man of Pedei (Pod'a)-tadba. This house is probably one of the village temples (Lennart Edelberg: Nuristani Buildings (in preparation)).

South of the village is a little grove. According to Jan Mohammad (Kafir name: Čimgal; information given the 24th Oct. 1953) a male god by the name of $W_{\text {thom }}{ }^{18}$ was standing here at the east end of a rectangular site girdled by a row of stones. At the eastem end now only a ruined heap of stones is to be seen.

The idol was naked, the penis visible. People from all the villages in Parun came here to sacrifice animals in the month of Wușum-ust $\tilde{u}$, at which time there was still some snow. A cow or a big male goat was sacrificed. Blood was thrown on the idol. The meat was divided and carried away to every house. The people prayed that the flocks and herds might increase and that milk might become plenty. If a woman was childless she might pray for a child.

I would propose as an obiter dictum that KK 11 might be identical with Wuşum (cf. Lennart Edelberg 1960a, p. 278 and Ahmad Ali Motamedi and Lennart Edelberg 1968 p. 5, footnote 1).

17 Morgenstierne 1949 p. 283: 'Ușum = (Kati) Šandē.

18 My notes lave 'užum' (cf. map). G.B.: Wușum,. 
V. Pronz (Paruni: S'äici); 46 houses (fig. 15):

Rob. 1896 p. 411 : "Saranji is the tutelary deity of the village of Pontzgrom [Pronz]. She has a little shrine on the top of the village tower, and a second near the mouth of the Pontzgul" (probably Pes-Gol on the map here).

Morgenstierne 1949 p. 283 and 1951 p. 163 identifies Saranji with the Parun goddess Sïlm'eč.

Abul Gheni in Pronz, who was a boy of twelve years at the time of the conquest of Kafiristan in 1896, gave the following information to me in 1953: Silmix is a goddess in Pronz. Her wooden statue was not bigger than a man could lift. Her breasts were visible. The statue was situated in the lower floor of the village tower (this tower was called Silmix, too). The upper floor was empty. The statue was in the milldle of the room. Around it were stone seats. They brought meat to the statue and threw blood on it. They sang in the room and played the drum (dad) and a double flute ( 2 persons were playing logether). But they did not dance inside the room, only outside.

There is a little uncertainly as to whether the existing tower is identical with the Silmič-tower. But as far as I can make ont, the reason for this is that the tower has been renewed between 1948 (fig. 15) and 1953 (fig. 16). In 1964 the upper floor had been removed and the lower floor had become the private property of a man who used it as living quarters.

1. The stone of Ashpegra (G. B.: Espereg-rā), now used by the mulla when calling to prayers (fig. 17).

2. The stone of Guduza ${ }^{19}$ outside the winter stables (fig. 16).

3. A tiny stone house built over a little brook (probably now used for bathing). It is said to be identical with the house in which Ashpegra met the old woman and her seven sons.

4. Gumra-kot (fig. 18), a stone on which oflerings of wheat and butter (the mixture is called: $e s ̌ r ̌ s)$ are placed, when threshing is finished on the last day of the month of ki-la.

1ө Morgenstierne 1949 has: g'ūtu = "cow" and zõ = "cow's dung". 
VI. Shtive (Paruni: Șup'ī); 80 houses (fig. 19):

1. The house of Kusum-ladba = the temple of Marra (fig. 20 and 21).

2. The house of Wäci-tadba = the temple of Disni (cf. Ahmad Ali Motamedi and Lennart Edelberg 1968: A Kafir Goddess).

3. Diibu-i.e. the house for birth and menstruation,

4. Irrigation channel made by a "dewa" (fig. 19) (cf. Rob. 1896 p. 410 about Dizane [Disni]).

w. Water mills.

In Shtiwe there were eight clan houses (actually lineage houses). I have only had time to locate two of them.

Rob. 1896 p. 380 "Large tracts of fertile lands [in the Partu Valley] lie undisturbed by the plough, because they are consecrated to Imrá."

Ahmad Ali Motamedi, diary 1953 p. 92: Outside the village of Shtiwe is a special field called sacii consecrated to the god [Māra?]. The harvest from this field is brought to the "leader" of the village, who is considered to be the son of the god [cf. myth no. 11]. On certain occasions and in connection with processions the harvest is thereafter distributed among the poor people (Persian: kheirāt).

Farther up the valley are different ruins of holy places:

Siĭlum iiyü (Persian: "bury bud").

Disni kslu which had no roof, but had two stone towers.

Zivud where myth no. 14 appears to have taken place.

Urtā, the junction of Ichemgol and Wiiäsgol. Here was a holy place for Māra. The grove there is probably still holy.

Düžü-Mãra is to-day only a heap of stones. The Kafirs sacrificed cattle here.

The lake Kermang Sur on the way up to Minjan has connection with the god Māndē.

'The girl Suga or Sämsuga (Simsuga), the daughter of the first Kusum, is connected with the Ichemgol. In the upper part of this valley is the holy lake Chening Sur (placed on my map according to oral information). 
VII. Dïröshwa is, as pointed out by Professor Georg Buddruss, the seventh, but now deserted, village of the Parun community. It is indicated on my map according to the oral information I received about it. The inhabitants, it is said, had moved from there before the conversion to Islam in 1896 for climatic reasons. The snow-fall was very great and the thaw started too late in the spring, so that the growing season was too short to support the population. Therefore they moved to "Aspatal" in Chitral.

In the upper part of the Wüäsgol lies the very important holy lake Sijyum Sur, near the watershed.

\section{Myths \& Hymns 1-22.}

No. 1.

Informant: Amir Shah (fig. 22).

Locality: Pashki.

Date: 25th of May 1948.

About the informant: Amir Shah was one of the blacksmiths living on the south-eastern edge of Pashki. He was black-clad (Siah Posh) and had three wives, one from Kamdesh, one from Wama, and one from an unverified place, but in any case not from Parun, from where he was not allowed to take a wife, because he was a "bari" (craftsman). ${ }^{20}$ - The other blacksmith was Gul Mohammad. They both belonged to the Zikia tadba.

This story was told in Persian to my German-speaking interpreter:

"Many years ago a father and his son, both blacksmiths, went from Kamdesh to Shtiwe. Here they lived in a tower-like building $^{21}$ and the slag from their iron smelting is still visible in Shtive. People were afraid of them and never took the manufactured ironware directly from their hands. The smiths had to lay out the ironware on the ground and sprinkle it with earth or dust. The recipients sprinkled it with earth, too, and not till then did

${ }^{20}$ Cf, Jones 1967, pp. 39-44.

21 'This butding was situated a litte farther up the valley than the vllage. 
they take the axe, the knife, or whatever it might be. ${ }^{22}$ One night the son woke up and told his father that there was plenty of clust, but his father said that he thought it was a mouse rummaging about. This repeated itself for three nights. In the morning, after the third night when they woke up, the house had moved down to Pashki (cf. fig. 24 and map, Pashki no. 5, where the blacksmith has a goatpen today). From these two men the blacksmiths are descended." ${ }^{23}$

No. 2.

Informant: Amir Shah (cf. no. 1).

Locality: Pashki.

Dale: 25 th of May 1948.

Told in Persian to my German-speaking interpreter:

"When the blacksmiths in Pashki had increased in number (up to 80?), they were destroyed. ${ }^{24}$ At dance feasts the blacksmiths beat the big drum. The blacksmiths had no land. But in the mountain northeast of Bargal ${ }^{25}$ they had gone and built some pens and cultivated some land.

Finally there was only one blacksmith left, and he was in the mountain pastures to watch the bull, the cows and the goats.

Now they were going to have a feast for Imo in Pashki. But for the dance they missed the drummer. "Where is he?" asked Imra. "In the mountain pastures!" was the answer. Then Imra went up there and asked the blacksmith why he did not beat the drum. Well, if the god would look around he could see that he, the blacksmith, had to watch the cows and flocks. Then

\footnotetext{
${ }^{22}$ Cf. Rob. 1806 , p. 385 , myth (3): "... At the bottom of the shallow hole made by the goat a knife was revealed."

${ }^{23}$ The scholar who eventually goes to Parum, should try to trace the genealogies of the blacksmiths, and in any case the names of the father and his son (G.B.: The names of father and son were known to none of my jnformants).

24 Whether they were destroyed by supernatural forces or by other people, $I$ did not find out.

${ }^{25}$ Cf. map. Bargal is perhaps Barigal. The craftsmen of Nuristan are called "barl".
} 
Imra transformed the bull into a big red stone and the cattle into smaller stones-and the blacksmith went down and beat the drum for the dance," 26

No. 3.

Informant: Abdur Rahim, Istan-tadba (fig. 25).

Locality: Pashki.

Date: 27th of October 1953.

Tape-recording: Pashki 8.

About the informant: Abdur Rahim was probably born about 1915 and is identical with A. in: Motamedi, A.A. and Lennart Edelberg 1968. Since about 1953 he has been malik of Pashki, i. e. the official spokesman of the village. He is active, agile, artistic, intelligent, and the leading figure especially during performances of music, song, and dance.

Text (according to informant not pure Paruni, ${ }^{27}$ but probably with strong influence from old-fashioned Kati):

Dangamser $=$ Calcutta

Alaser $=$ Polyatak $=$ Pul-i-Allock?

Kama = Kama northeast of Jalalabad

Närink = a village near Chiga Serai (Närai?)

Yudu = unknown

Sherkh = name of person

Dewa $=$ Dewa in the valley of Parun?

Kate $=$ Kantiwo

Rananguye $=$ Kantiwo

"Mekka Medina obya pafǔ̌

In Mekka and Medina occurred

\footnotetext{
26 The stones, it is said, can still be seen in the mountain pastures on the right side of Bargal.

27 G.B.: In 'Text nr, 3 I cannot find a single Paruni word. The text is an example for the typical gibberish of many Paruni songs. Was the language intentionally corrupted, so that it could not be understood by profane ears? In 1956 one of my informants from Dewa called this sort of speach "language of the Gods"1
} 
Mekka ete naftya papuš ${ }^{23}$

a distrust (among the people)

bimserate Damgamser

so they went to Damgamser

adiaš Damgamser

they came to Damgamser

ata naftya guš ${ }^{28}$

again they travelled(?)

bimserate Industan

and went to Hindustan

adiaš Industan

and came to Hindustan

naftya guš

distrusting (each other)

bimserate naftya guš

they went they travelled(?)

Ataser adiaš

to Ataser they came

bimserate Kama

they went to Kama

adiaš de naftya guš

they came there, too, they quarrelled (botween each other)

bimserate Närink

they went to Nārink

šio tanaš

they reached (Nārink)

as There seems to be some confusion as to the meaning of "naftya papus" and "naftya gus". 
naftya papuš

they were unhappy

Yudupase adiaš

to the mountains of Yudu they came

Šakanešata

they began to fight

naftya guš

they were not glad

bimserate ba Dewa

they went to Dewa

šu tanaš

they reached there

motan papuš

they were working there

nui brok šanus papuš

they were making butter by using the skin

Kate brok ša papuš

off Kantiwo they drove the women (to the upper parts?)

oral patašete

they went high

šiu tanaš

they reached (high)

Rananguye šio tanaš

the village of Kantiwo they reached

wošanpase brau zieš

the horse they mounted

ošanpase ganto wanaš

a horse statue(?) they made 
dumi paseda ašinta tawaneš

tail side grave (tomb) they made

pakum manu šinba (= sị b $\tilde{\bar{a}})$ tawaneš

neck-side chair (horn stool) they placed

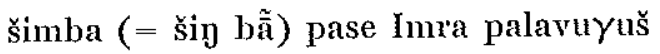

hor'n stool Imra went up sat down

No.4.

Informant : ?

Locality: Pashki.

Date: 31st of May 1954.

Tape-recording: Pashki 18.

Free rendering:

Seven to eight of the bravest Kafirs tried to reach the lake and their aim was to catch a man who lived in that lake.

After many efforts they reached the lake. Then they discovered that the lake was entirely frozen. And they saw the man sitting in the lake with ice up to his waist.

They were happy and hurried to catch the man, but the ice broke and the water came up and they all drowned. ${ }^{29}$

\section{No. 5 .}

Informant: Wodi $=$ Ali Khan.

Locality: Kushteki.

Date: 26 th of October 1953.

Tape-recording: Kushteki 8 .

20 Schuyler Jones in a private communication: "Many of my Waigali stories about urey (silver cups) involve the need to control the spirit of the lake. This is usually a calf rather than a man. The Waigall people say sorotsala. He who controls this spirit can remove treasure from the lake. All these lakes are believed to contain treasure: urey, sōnurey (golden cups) and "all the furniture for a house made of gold". But one must be able to control the spirit of the lake or one is pulled into the water and drowned. The theme of your story where the men try to catch the man in the lake and drown in the attempt, is also the theme of many Waigall stories." (Cf, no. $11 \mathrm{~A}$ and $\mathrm{B}$, no. 13 and no. 14). 
About the informant: Kafir name: Wodi, Islamic name: Ali Khan. In 1953 about 80 years old and blind. As the young men of Kushteki were laughing when he sang, he was brought inside the guest house where the tape-recording was made and where he accompanied his song by gesturing towards the imaginary statue of Imra in the north-west corner of the ruined temple (ef. fig. 26). In his youth Wodi had danced in the chief temple to Imra in Kushteki and sung this song, which was used in the summer in the months of ü-la and nila-la (nü-la).

Paruni text:

prấčok, prẫčok

kiwărok'ōroko, kiwärok'ōroko,

prî̉cok, prấčok

waleǰili kumliwari kōrokō

hač

Translation by help of Abdur Rahim, Pashki:

prấčok = bravol (when the dance was specially good, they shouted this word).

kiwärok'orroko = to play the flute

walejili $=$ (Persian) zud kun $=$ hurry up!

kumliwari $=$ (persian) Jam $=$ join together!

hač $=$ hurray $^{30}$

No. $6 \mathrm{~A}$.

Informant: Abdulla from Pronz.

Locality: Pronz.

Date: 30th of May 1954.

About the informant: Abdulla (cf. fig. 27) was in 1954 a man about 40. He died (probably of tuberculosis) some time in the decade up to 1964, when I met his son, a boy 15 years old. Abdulla told this story sitting on the riverside south-east of Pronz and accompanied his statements by pointing to different

\footnotetext{
so The older Abdur Rahim in Pashki (not the informant) used when listening to this recording the expression "tue" instead of "hat".

G.B.: 'Text 5 is not in the Paruni language.

4 Acta Orientalla, XXXIV
} 
places around us. The story was told in Persian and translated into English by student Moh. Karim Nushin, now chief physician of the Malaria Hospital in Kabul.

To help the reader I have tried to make a compilation of the persons mentioned:

Wäči (and her seven sisters), mother of Ašpegra.

Ašpegra, ${ }^{31}$ son of Wäči, married to "daughter" of "old man". Yini, mother of Guduza.

Guduza, ${ }^{32}$ god who develops the calle, son of Jini.

An "old woman" (I).

An "old man" with a "daughter", later married to Ašpegra.

An "old woman" (II) (= Purdi), mother of seven sons.

Seven sons of an "old woman" (II).

A Giant.

Imra, chief god of Parum = Yamrei. He corresponds to Māra

(cf. Morgenstierne 1949 p. 283 and Morg. 1951 p. 163).

Once there was a fairy (Wä̌i) and a jin (J̌ini). ${ }^{33}$ They were two women and lived as sisters in a house on that hill [informant pointed at the têpe of Pronz]. Both of them had a son. One day they were sitting outdoors and while they were talking of different matters, the jini said to the fairy: "We have lived and loved so long as sisters. My last wish is that if I die, you should take care of my son [Guduza] as though he were your own, and if you die, I promise that I will take care of your son [Ašpegra] as my own."

The name of the fairy was Wäči. ${ }^{34}$ WVäci died. Her son remained alive. J̆ini was training the two equally and was feeding them equally.

One day an old woman came to J̌ini. She said: "I want some

31 Cf. Snoy 1962 p. 86.

s2 Morgensticrne 1949 has: g'uṭ = 'cow' and zõ = 'cow's dung'.

s3 G.B.: Paruni fini is a special class of supernatural beings and has neither historically nor linguistically any comection with Arabic fin. The similarity of sounds is mere accident.

31 G,B.: "The name of the fairy was Wäil": This is clearly a misunderstanding of the interpreter. Wati is no proper name, but a special class of supermatural beings, similar to the "fairies". (C.., however, no. 15). 
fire." Jini was angry with the old woman and said: "I haven't got any fire. Go back to your home!"

'The old woman said: "I had a secret, a good secret, which I wanted to tell you. Well, if you don't give me fire, I am going to my home,-and you go to your home."

The old woman went quickly. Jini was surprised at the old woman's secret. She quickly took bread in one hand and fire in the other hand and went after the old woman calling. The old woman saw her and stopped, took the fire and bread and said to Jini: "What are you doing? Why are you training and feeding Wäči's son like your own son? 'Take him away and keep your own son well."

That night Jini was thinking and at last she felt that the old woman was right. "In any case this boy is not mine."

Next day she separated their dishes. She gave wheatbread to her own son and bread with ashes to the son of Wäči.

On the first day the poor boy took the ashbread and went to the grave of his mother. He put the ashbread on the grave and wepl: "Oh, my dear mother, see, they are giving your son ashes to eat."

A voice called from inside the grave: "My dear' son, don't be sorrowful. Leave these loaves here and go. Go to that cow-shed [informant pointed at the winter-cow-shed west of the mosque and near to the stone Guduza, cf. map] and there is a cow. Go to her. You will find everything."

'The boy did so. As he saw the cow in the cow-shed the cow stood on her feet, respected the boy and excreted, but it was not cowdung. ${ }^{35}$ It was lumps of cheese. The boy ate the cheese. In this way the boy grew big and well-fed.

Every day the two boys were playing near the village. They went to play at shooting arrows.

One day when they were playing, the son of Jini pointed at a

3s Schuyler Jones writes in a private communication: "This reference to the cow and cowdung is an interesting theme. Waigali myth account for the origin of the sewala (a low-status class of craftsmen) either by explaining their descent from a cow, or by saying that they emerged from cowdung. According to these myths of origin, there were two brothers: Mir Kola, apical ancestor of all sevala, and Mir Sin, apical ancestor of all Hindus."

$4^{*}$ 
white spot and said to the son of IVäci: "Come, we will shoot at that spot."

But Wäči's son refused to shoot and moreover he said to Yini's son: "Don't shoot!"

The son of Jini did not care and shot at the spot with an arrow. 'They saw handfuls of ashes, and when going there they saw the ashloaves. The son of Jini said: "What are these?"

Wäči's son said: "These are the loaves your mother has been giving to me."

Yini's son was astonished at this. He said in a sad voice: "To-day you shall sit on my chair, and I on yours. If it is true that my mother has been giving ashbread to you, I will kill my mother. If it is not true, well, then it does not matter."

They went and took their seats on the chairs, as they had said before. The mother came with the bread. She put the ashbread before her own son and the wheatbread before Wäci's son. Jini's son asked his mother: "What is this?" He jumped to his feet and hit his mother. Wäči's son seized the boy and prevented him from hitling his mother, and they went out.

The old woman came in and told the old true story to her, the story about how Wäci's son went to the grave of his mother and how he was eating the cheese from the cow and how he was growing and getting strong. "Well, I will show you what you can do. You shall pretend that you have a severe pain in your abdomen. Crouch down and when they come, say that you have this severe pain and tell them that the medicine for it is cow meat. Then say: "Go to the cow-shed and kill that cow and prepare some meat for me."

The son of Wäči said: "All right, we will bring it."

The son of Jini said: "No, we will not let the cow be killed, but let my evil mother die of this pain, so that she may see what she has done to you, and then we two brothers will live happily."

But the son of Wäci went to kill the cow. But to his surprise he saw that the cow was not there. They followed the footprints of the cow up in the valley. They went to a certain place in the valley. 'They lost the footprints of the cow. Near the bridge [informant explained: the bridge over the stream from Pesgol] they slopped. The son of Wäci went to the Pesgol valley. He found 
the footprints of the cow. He went on and on till he came to a green meadow [pasture land = Paruni: muns]. He saw the cow grazing. The cow looked angry and said nothing. Wäči's son apologized, and they became friends again, but the cow gave no more cheese. It was only cow-dung.

The cow said: "Go up the valley. There are seven sisters of your mothers who live there."

He went there and stayed some years with his aunts.

One day he said: "I want to go to the village and see how Yini and my brother are."

His aunts gave him a cummerbund [Persian : kamar-band] and said to him: "If you see that your cummerbund becomes red, then come back soon."

The aunts placed a cup of water before themselves and said: "If the water here becomes red, we will come after you."

Wäči's son went and sat down under a walnut tree refreshing himself. He saw an old man carrying a young and beautiful girl on his shoulders. The son of Wäci and the old man greeted each other.

The boy said: "Where are you going?"

The old man said: "There is a big giant. Every year we have to give him a young girl otherwise he will destroy our village."

The boy said: "I am young and strong. You are old and weak. I will bring this girl for you to the place." The old man stayed there. They (the boy and the girl) went up and came to a lake. The giant came out, the girl was afraid.

The boy said: "Have no fear!" He took an arrow and shot it into the heart of the giant. The giant fell and was dead. The boy came back with the girl. The old man admired his bravery and said: "Now you should shave the hair from your headl" And while the old man was shaving him, he cut off his head.

The old man and his daughter came to the village. They rejoiced and were respected very much by the people.

The aunts of the boy saw blood in the cup and they came and took the boy to their house and cured him and he was alive again.

When he was all right, he said: "Now I will go and take revenge. I will go and kill that old man in Pronz." He saw the old 
man sitting on a čarpoi [a bed] clad in new garments. Boys and girls were dancing before him. He saw the old man's daughter weeping and singing: "What was the fault of that young and brave boy whom you killed?"

The boy came and killed the old man, her father, with an axe. His daughter told the whole story to the village and assured the villagers that the boy had killed the giant. They respected him and placed him on the seat ${ }^{36}$ of the old man. He married the girl and lived happily in the village.

One day he saw smoke. ${ }^{37}$ He said: "What is that?"

His wife said: "That's a place from which no one returns alive."

He was surprised and went towards the smoke. There was a house and he went inside. He saw an old woman. ${ }^{38}$ She had made her knees like a tripod. Her left hand was like fire and her right as a spoon. She stirred wilh her spoon inside her stomach cooking something.

"What are you doing?" he asked. "From where have you come?" asked the old woman.

He said nothing. He went, brought a tripod and wood, made a fire, placed a stone pot on it and gave a wooden spoon to the old woman and said: "Isn't it good?"

The old woman was glad and said: "Now you have done so much for me. I would like to save you. I have seven sons, strong, bad-tempered and with bad complexions. If they see you, they will eat you, so go at once, because I am afraid."

36 I wonder whether the informant has meant a "horn"-chair (cf. fig. 33),

a From here consull Rob. 1896 p. 385 (story 3) and p. 387 (story 8).

Professor W. Lentz has proposed (oral conmunication) this myth and no. $6 \mathrm{~B}$ may be comected with the winter solstice, when Imra is saving the Sun from the evil demons. Cf. in this commection Akten des 24sten internationalen OrientalistenKongresses, Munchen 1957 p. 515 , where Lentz proposes as the function of the chief temple to Imra at Kushteki "to serve as a sun observatory in which the flrst beam of the rising sum on the winter solstice was to hit a statue of the god inside of the temple and, thus, to indicate the beginning of the New Year".-This statue appears to be the same as the statue KK 1 in Musée Guimet, Paris (cf. Edelberg $1960 \mathrm{a})$.

${ }^{28} \mathrm{Cf}$, no. $6 \mathrm{~B}$, where the old woman direetly is named "Purdi" by the Persianspeaking informant (cf. Morgenstiene 1949 p. 269). 
The boy said: "I will not go. If you leave me for them to eat or if you hurt me, it is up to you. I haven't got any place to go."

'The old woman said: "Now they are coming. Go and hicle yourself. 'They are each bringing a markhor."

The old woman said: "The eldest will come in front of all, You should say: manda na bashil And then, if he says: zinda bashi $l^{39}$ it is all right. You can come out. Bul if they say nothing, they will kill you and me."

The sons came, they went inside. The boy said: "Mainda na båshi!" 'The eldest said: "Zinda bashi!" in reply. "From where have you come?"

The boy said nothing. They ate their game and the old woman said: "He has done so much for us, he has brought a tripod, made a fire, brought a stone pot and a spoon."

They were all glad and went to sleep.

In the morning when they were leaving the house one of them said: "You can eat everything in this house. But you should not go to that wall, and you must not touch that shutter."

They went out, and the mother also went out. The boy went to the shutter and put his finger into a hole in the shutter. When he took it back it was all gold. He could not restore it.

The old woman came and saw the golden finger. "What have you done? In the evening when my sons come home, they will kill both of us."

She wrapped a piece of cloth around the finger and said: "If they ask, you should say: I have cut my finger."

In the evening when the sons came, they saw the wrapped finger and said: "What have you done to your finger"?"

The boy said: "I have cut my finger with a knife."

The next day when they went out again, the boy went to the window and put his whole forearm into the hole of the shutter, and it was all gold when he took it back.

When the old woman saw this, she said crying: "You have brought us bad luck." She wrapped a big piece of cloth round the whole forearm. In the evening when the sons came, they

s9 The common (Persian) grceting in Afghanistan = May you not be tired May you livel 
asked again: "What is the matter?" The boy said: "It is the wound of yesterday which has caused all my arm to swell up."

The next day, when all went out again and the mother too, the boy went to the shutter, forced it with his two hands and got it open. He went inside. He saw a pool. He saw a horse in the pool. On the right shoulder of the horse was the Sun, on the left the Moon.

He went and took out the horse. The old woman came. She saw it and cried loudly. But the boy rode the horse and went to the sky.

The seven sons on the mountain siles saw that the sun had risen. They were surprised and came immediately to the house. They saw that the boy had taken the horse and had gone to the sky. The sons wanted to kill the boy. The boy said something to the horse. The horse said: "Look into my left ear. If you do not find anything in my left ear, there is a sword inside its sheath in my right ear." 40

The boy look the sword and killed all the seven boys. He gave the Sun and the Moon to the world, because Imra had told him to do so.

Imra and Ašpegra were good and close friends after that.

Imra was in Kushteki.

No. $6 \mathrm{~B}$.

Informant: not stated-probably Abdulla (cf. no. $6 \mathrm{~A}$ ).

Locality: Pronz.

Date: October 1953.

No. $6 \mathrm{~B}$ was told in Persian to and written down by student Ahmad Ali Motamedi, now general director of Antiquities in Afghanistan. Translated by Moh. Karim Nushin (cf. no. $6 \mathrm{~A}$ ).

Il is remarkable that the son of Wäči is called Eshpegra (= Ašpegra) from the very beginning of the story. Purdi, which is Paruni meaning "old woman", is here used as a proper name for the old woman II.

40 Cf. Rob. 1896 p. 385-6, story 3, and p. 387, story 8. 


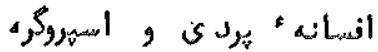

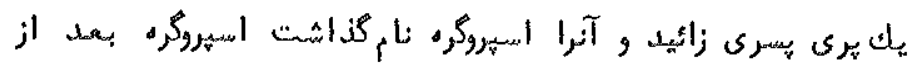

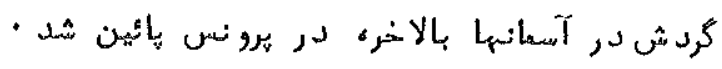

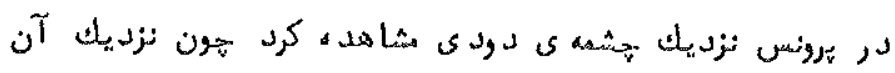

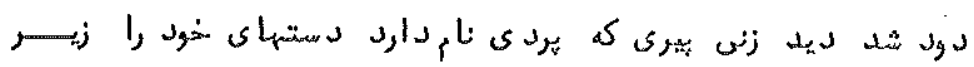

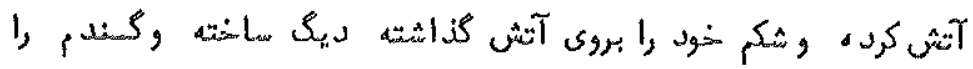

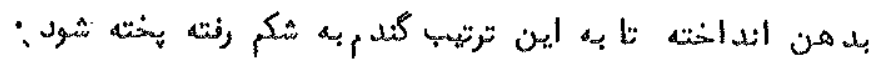

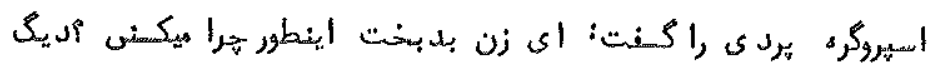

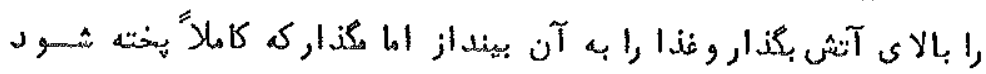

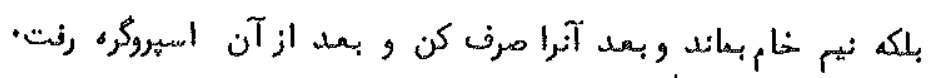

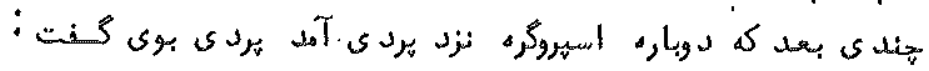

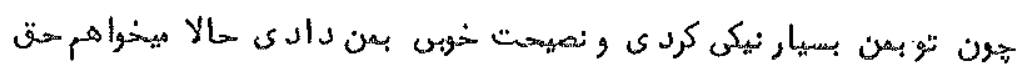

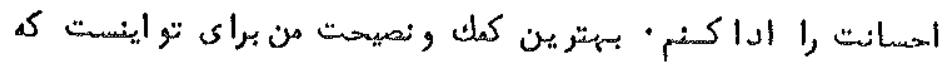

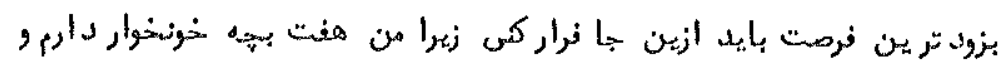

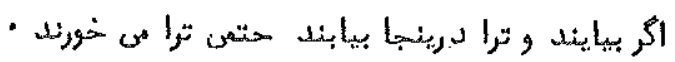

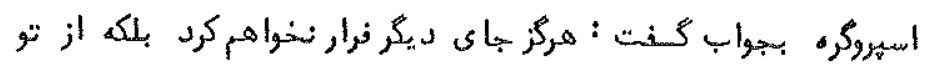

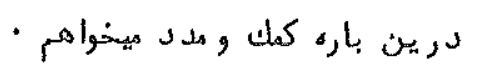

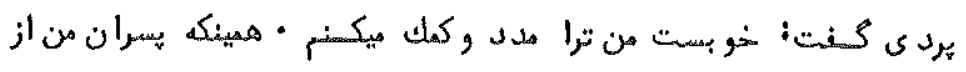

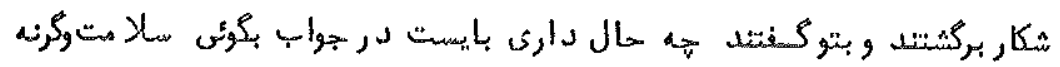

$$
\text { ترا هي-نورند }
$$

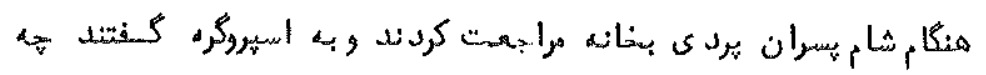

-

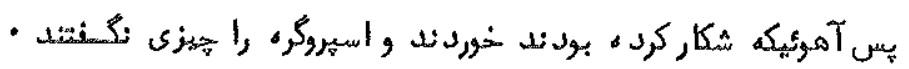




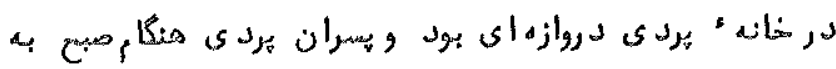

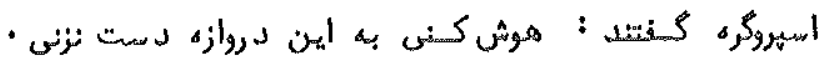

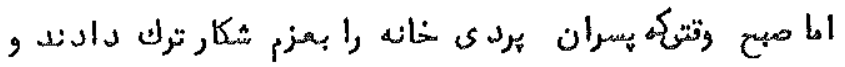

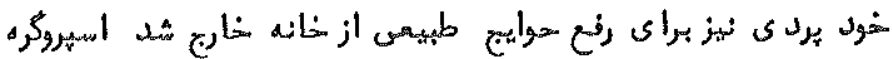

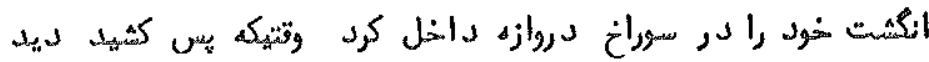

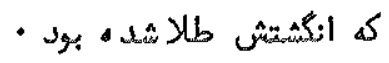

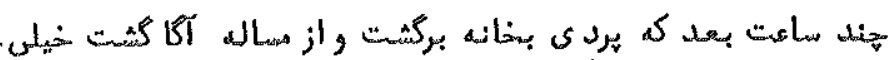

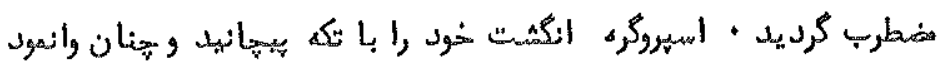

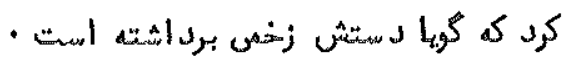

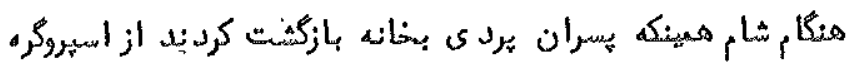

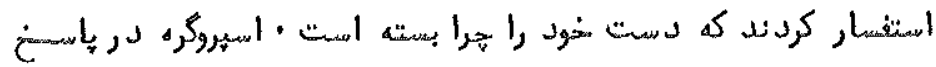

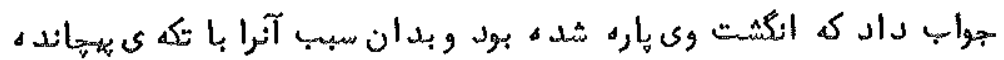
المست

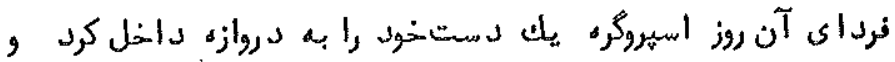

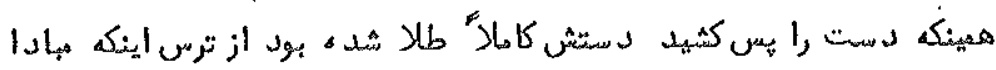

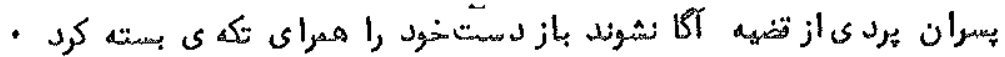

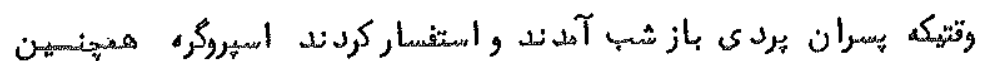

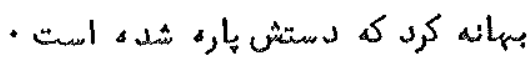

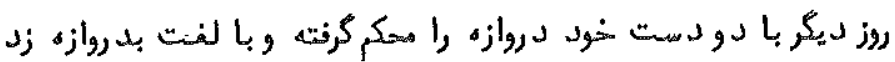

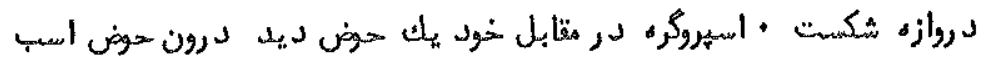

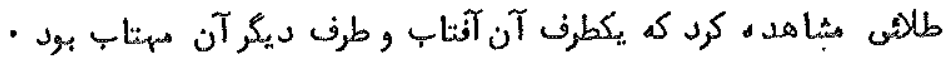

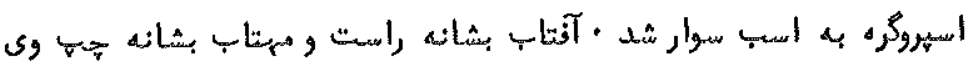
تراركرفت وتتيك هيران تردى آمدند واين هنظره را تماشا كردند متهير شده بناى 


$$
\begin{aligned}
& \text { - }
\end{aligned}
$$

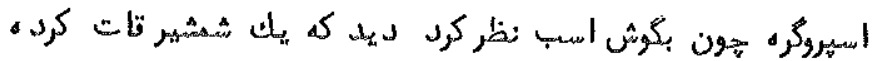

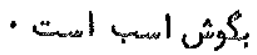

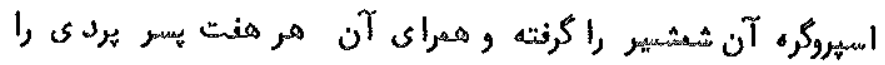

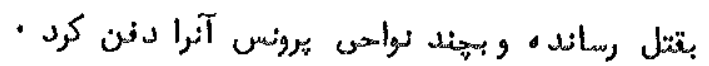

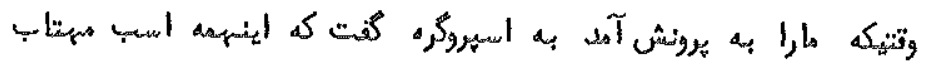

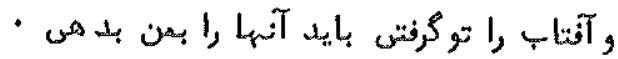

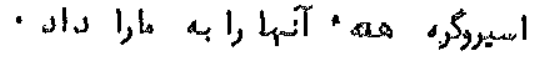

The story of Purdi and Eshpegra (Eshparogra):

A fairy delivered a son and named him Eshpegra. Eshpegra after travelling in the skies at last came down to Paronz [Pronz]. In Pronz he saw smoke near a spring. When he was near the smoke he saw an old woman named Purdi putting her hands under the fire and using her abdomen as a pot and putting wheat into her mouth to go into the stomach and be cooked there.

Eshpegra said to Purdi: "O, unfortunate woman, why are you doing that? Place a pot on the fire and put your food into it and leave it to be half cooked and then eat." Then Eshpegra went, and when he after some time came again to Purdi she told him: "As you have done good to me and have advised me so well, I want to pay the debt your goodness has incurred. $\mathrm{My}$ best alvice to you is that you should go as soon as possible from this place, because I have seven savage sons, and if they come here and see you they will eat you."

Eshpegra said: "I will never go anywhere else but I ask help from you."

Purdi said: "Well, I will help you. As soon as my sons return from hunting and if they ask you: How are you? you should say: I am all right. Otherwise they will eat you."

In the evening they came back from hunting and asked Eshpegra: "How are you?" He answered: "I am all right,"

So they ate the deer they had hunted, and did not say anything to Eshpegra. In the house of Purdi there was a gate and in 
the morning the sons of Purdi told Eshpegra that he must not touch that gate. But in the morning when the sons went out hunting, and Purdi herself went out of the house to do some necessary things ...., he put his finger into the hole of that gate and when he drew it back it [had become] gold.

Some hours later when Purdi returned home and was aware of the problem, she became agitated. Eshpegra wrapped his finger in a piece of cloth pretending that he had wounded his hand. In the evening when Purdi's sons returned home they asked him why he had tied his finger with the cloth, he told them that he had wounded his finger and that is why he had wrapped it.

The next day Eshpegra put his whole hand into that place and found it made all of gold. Out of fear for Purdi's sons he again wrapped his hand with a piece of cloth and again when they asked: "What is the matter with your hand?" he pretended the same as before, that he has wounded his hand.

The next day Eshpegra took hold of the gate with his hands and broke the door with his foot and he saw a pool in front of him. In the pool there was a golden horse and on one side of it was the Sun and on the other side the Moon. Eshpegra mounted the horse and the Sun was placed on his right shoulder, while the Moon was placed on his left shoulder. When Purdi's sons returned and saw this, they began to fight. Eshpegra saw that there was a sword hanging near the ear of the horse. He took the sword and killed all the Purdi's sons and buried them in dillerent areas of Pronz.

And when Măra" came to Pronz he told Eshpegra: "You have taken the horse, Sun and Moon; you should give them to me." Eshpegra gave them to Mära.

No. 7.

Informant: Young man.

Locality: Pronz.

Date: 21 st of October 1953.

Tape-recording: Pronz 6 ?

41 Māra corresponds to Imra (cf. Morgenstierne 1949 p. 283 and Morgenstierne 1951 p. 163$)$. 
Paruni text (noted by Ahmad Ali Motamedi in Arabic letters):

1. watma guishuk žakolem karutāa

2. aomash guishuk žakolem karuțā

3. zino guishuk žakolem karutä

4. kam guishuk sakolem karutaa

5. shabel guishuk bikelem karuła

watma $=$ Waigal, aomash $=$ Ameshdesh, zino $=$ Wama, $\mathrm{kam}$ Kamdesh, shabel = Minjan (five geographical places surrounding Partul).

guishuk = gyöş'ü = male goat.

$\check{z}$ akolem $=$ let us drive up (the valley). $\check{Z} a=$ up the valley.

sakolem $=$ let us drive to the west. bikelem = let us drive down. karuta $=$ G.B.: name of a well known hero of ancient times who among other things is said to have introduced the cult of the goddess Disni into Dewa. Disni in one story is said to have liked him especially and to have helped him in his warlike enterprises.

Translation by professor Georg Buddruss:

1. Let us drive up the valley the male goats of Waigal, o Karuta!

2. Let us drive up the valley the male goats of Ameshdesh, o Karuta!

3. Let us drive up the valley the male goats of Wama, o Karuta!

4. Let us drive to the west the male goats of Kamdesh, o Karuta!

5. Let us drive down ${ }^{42}$ the male goats of Minjan, o Karutal

Informants: Three old men.

No. 8.

Locality: Pronz.

Date: 21st of October 1953.

Tape-recording: Pronz 2.

Paruni text:43

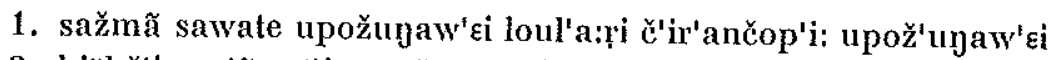

2. biubž'i saz'ãwat'è upožußaw'ei etc.

42 From the Minjan or Kamah pass.

48 I am grateful to Professor Georg Morgenstierne for his help when transcribing this song directly from the tape-recording. 
3. tišst'i saz'ãwat'è upožụ̂aw'ci etc.

4. Wuz'ul saz'ãwat'è etc.

5. 'übäc'uk (or: opošuk) sazãwatè etc.

6. tiz'i sa'zãwat'è etc.

7. didži (?) saz'ãwat'è etc.

8. telarex saz'ãwal'è etc.

9. ir'i saz'ãwal'è etc.

10. ozm'io saz'ãwal'c̀ etc.

Translation :

First word in each line = name of mountain pasture (Pashto: 'banda'). sawate, saz'ãwat'è $=$ near

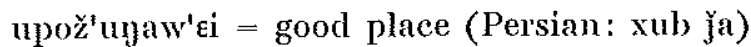
loul' $\mathrm{a}:$ ri $^{\mathrm{i}}=$ noise of rushing water from the mountains čir'ančop'i = strong noise of water; or: sound from churning butter in a goat-skin!

\section{Comments :}

1953: The song was sung in the temple of Pronz, which had been seen by one of the informants at least.

1954: The song was sung in the morning when churning butter, but is not used any more.

$$
\text { No. } 9 \text {. }
$$

Informant: Old man.

Locality: Pronz.

Date: 30th of July 1964 .

Tape-recording: $1964,81-82$.

Jan Mohammad, the malik of Shtiwe, explains the text in Persian:

amu derra dar'ūn rafta - nē eh - that valley which goes into (the mountains), you see

bãz lawistān māl mēbaran in the summer the cattle they bring 
sukun'at i banda dāian

livelihood in banda"s they have

ami jai bandara sīfat mēkunad

well - these places the 'banda' it [the song] qualifies

tar'îf mēkunad

it praises

felani jai itur xob jais(t)

felani jai itur xob jais(t)

felani jai itur xob jais(t)

that place such a good place is

ami čizi ki mēxānan "dur" "45 maid'ana mega

and, well, that thing which they sing (= call) 'dür' means pasture

felani maid'an felani maidan felani maidan

that pasture that pasture that pasture

ami namiš as $(t)$

eh, that is the name of it (the names of them)

Informant: Old man.

No. 10 .

Locality: Pronz.

Date: 30th of July 1964 .

Tape-recording: $1964,78-79$.

Jan Mohammad, malik of Shtive, explains the text in Persian: "tiz'ãn tiz'ãn" (in Persian: ura bibin)

look at that, look at that

ki "māali"

that (is) $\mathrm{Madi}^{46}$

4 banda (Pashto) = mountain pasture, sacter.

ss Morgenstieme 1949 p. 257: d'üru S. = flat. Lentz 1937 (in Deutsche in Hindukuseh) p. 322: go: d'ur = Weideplat $z$.

${ }^{40}$ Morgenstierne 1949 p. 283 : W. Mẫdé; Morgenstierne 1954 p. 319 : W. Mã̃d'è; Morgenstieme 1951 p. 164 : W. Mẫdé, Ashk. Mảdē ... His name is probably derived from *Măndē < Mahä(n)deva. 
yak pirišt'ara ki digar piš ba kufr såxtan an angle formerly in pagan time they made

pirišt'a såxtan

angle(s) they made

ura kal'ān mēbinan

that big they look at

ura mahab'ad mēbinan

that respectfully they look at

but as (t)

statue it is

ba Paski-s(t)

in Pashki it is

"tizon 'tizən" (=) ura bibinin ura bibinin

look at that, look at that

ura paraštiš kunin

worship do

ura tāzim (?) kunin

honour do

änna “tiz'ān tiz'ān”

well, look at that, look at that

ba nama alm'uz (?) ki degar

it shows (?)

u kal'ãn mēsãzan

that big they make

No. 11 A.

Informant: Noh. Alam, Pä'čog tadba and Zāmān.

Locality: Shtiwe

Date: 20 th of Oct. 1953.

Tape-recording: Shtiwe 3. 
About Zāmañn: Z., Kusum-tadba, is the younger brother of Kusum (fig. 21). Both are sons of the "Malik" von Schtive" (Deutsche im Hindukusch 1937 p. 136). Kusum is probably the formal head of the Kusum-ladba and master of the house in which the statue of Mara was situated. But Zāmăn ${ }^{47}$ appears to be a bigger man in the Parum valley than his brother.

Transcription noted directly from the tape-recording with the help of the mulla in Pronz:

are kzöm set piö lauzogla ateg listöpö undomišin towåt žizagla towåt žizama žipzi towåt silagrest šuwism dadäa užukta wourasa pseli winlara kürusum doš kür üžu pseli užuktakwarä ari šu ibzizm darä pseli warilamša atek abiyya banya bab ateg marštu atek ir atig nog telig abzumus sur irpöm atik nokso atek jomar muš üpšo"adea tilik ablia mås awo ${ }^{148}$ tilig ablia maya wari pseriraso bi iberisöm då iberi naucü läma ari walew warišir bi šilå labzi tešt labpogbazå užäzömdå zåt påzinč lapöndå atek öbiyya dübu ${ }^{49}$ såbzi sũndå dübu såbzidea žübüš låbzindå zübüčc oksåmaya ala birouloka abüča sorogost sočoromoya aso naskosim(r)då dütmüya baniabåb såbokso ai asa gal utakliji yu müsosolya iniždi nauskosinig laša laža limdå lyäma ölöra žiındöz alöra žinzama atek'ö lač wörifan ašlegså žinsameia wiš nableimdå tiisï aspeimdå waci'mi abručä ästlä bišüsömdå ästla wacï absäkså wacü absismdå labzi teš labsäkså wöz latogüsmdå

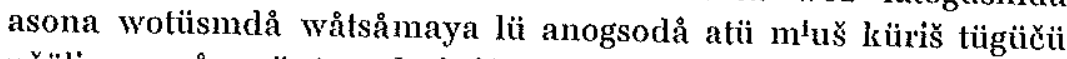
učöli asagså acü tamol tipåä sömdådäa atagagnoksodo tamol biti warga anogsodo tonusati (w)ürogså anogsodå gi ayürüisö ži'sti acä-äg tibzisö acä-äg žabsidäa ukurigčia tibsisö astla ünåsismdå istägså lyama usti lå mibi alåbizmdå alåbožibsi tak atek asurpan pebzismdå såpi pebzismdå asuranadia atek maidån pan bilårismdå iberismdå isla askusinokså sumara unzu usmdea ülüšpan tibzi obzokså tibzi obza unzusunda čiucu tibzi obzok så čiucu atek bịja oyeya čiucu alok čiucu smbok čiucu čilägså pözmazdä yu atös psöskåläšå ${ }^{50}$ masså alä usåsta päsäk äkdä

$\$$ C. Heath 1963 , Oct. $4 \& 5$.

48 Interpreter: 'awol' = to copulate.

49 dübu a deliverance house (cf. Morgenstierne 1949: zwāt $=$ birth house).
${ }^{60}$ possibly: psỏskảlała.

5 Acta Orfentalla, XXXIV 
öniš obliu limdå obliu lämaya ariwogsobi čilism ya atok sismdå ates wåsismdå åpia päzämamiš ayäya yupåsåma pir čiri lišižiö psö abliarasm ates psoblišå bašadå usåsta pesak äktä niš obliu limdå lyäma läždä äktä nägši abliagå ginåci gusåsta pägši piägäst yanan abinya üstågust ya atek išï wokså nan atek dui okså arinasira cuöri.

Translation into Persian dictated by the villagers in Shliwe to Ahmad Ali Motamedi (Oct. 1953):

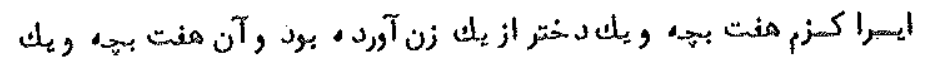

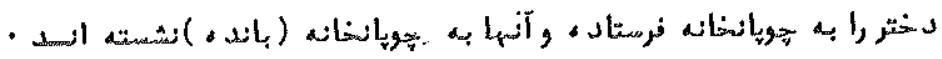

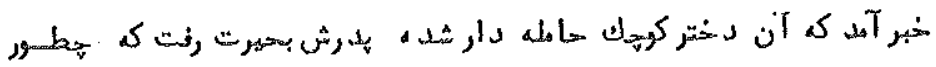

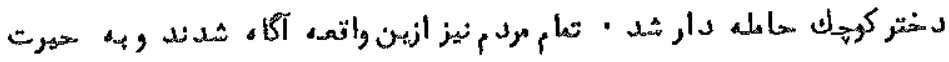

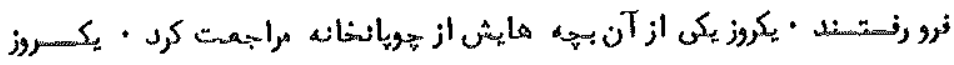

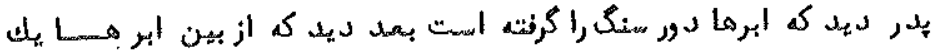

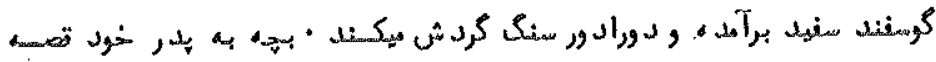

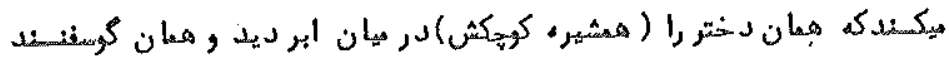

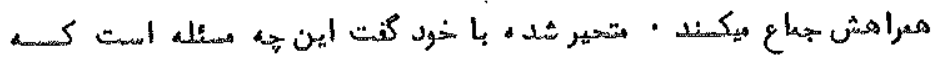

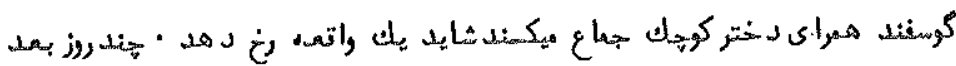

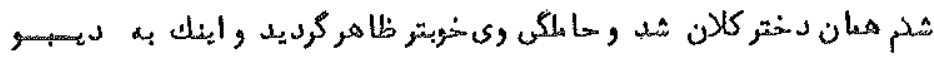

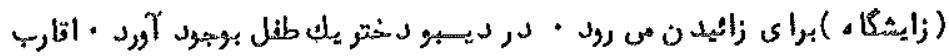

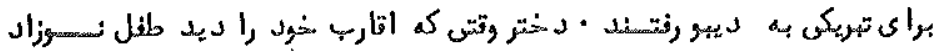

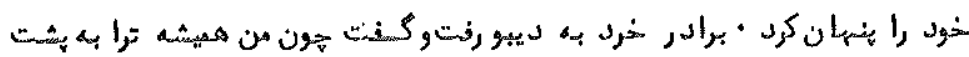

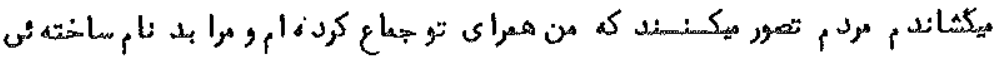

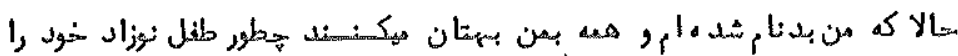

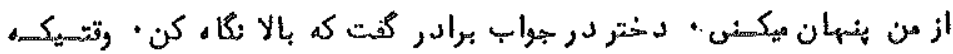

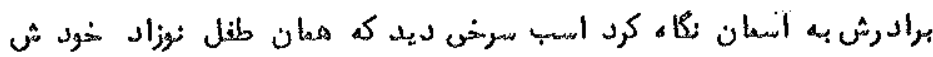




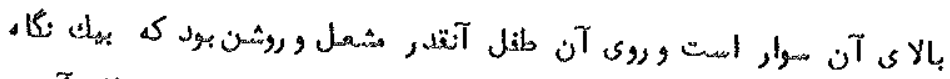

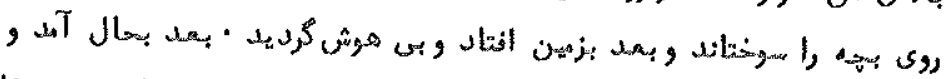

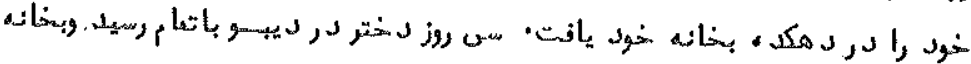

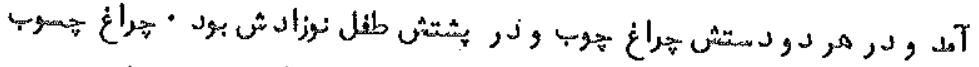

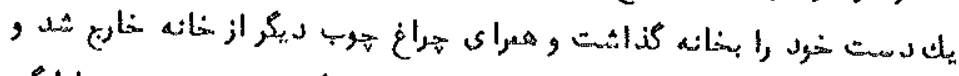

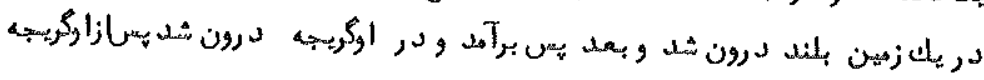

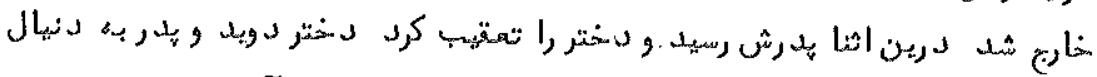

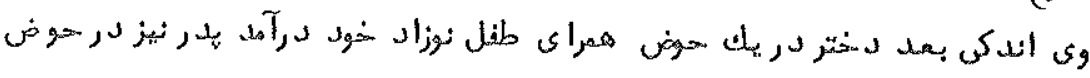

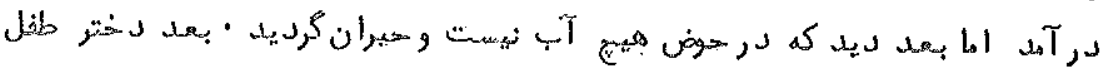

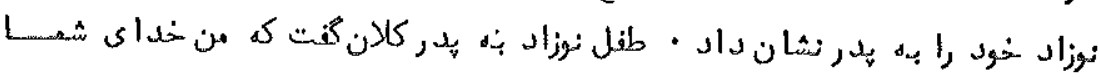

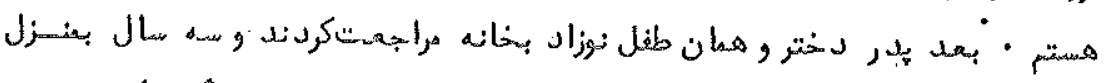

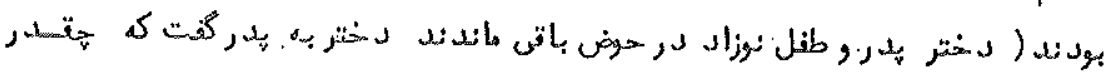

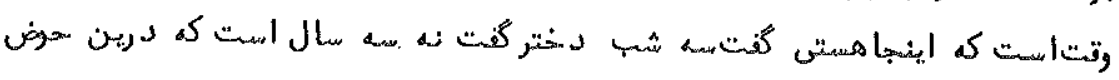

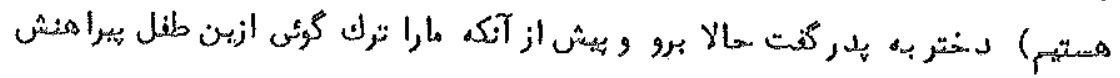

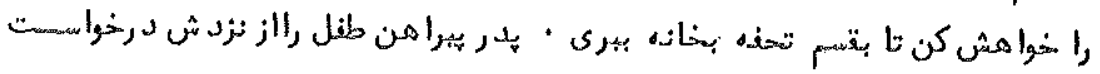

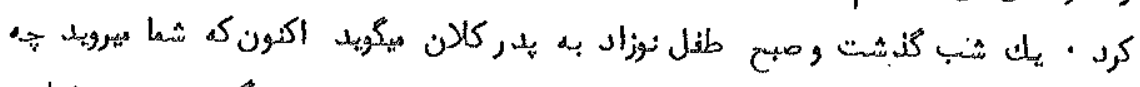

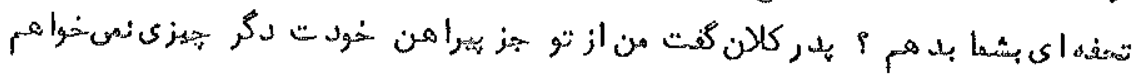

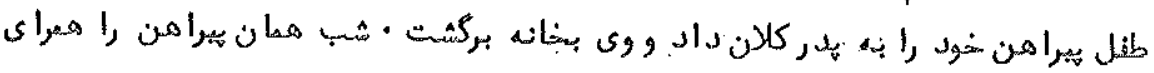

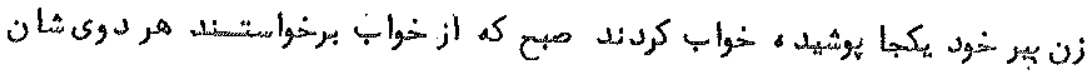

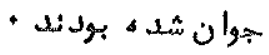

Transcription by Professor Georg Morgenstierne (wilh a few suggestions by Ulf Timmermann):

Îrā Kuzum haft bacca u yak duxtar az yak zan âvurda büd. Ū ān haft bacca u yak duxtar rā bà copān-xãna firistäd. U ānhā bi copān-xāna (bānda) nîšasta and. Xabar āmad ki ān duxtar-ikücik hămila-dār šuda. Pidar-iš bi-ḷairat raft ki cị̣ūr duxtar-i- 
kücik hạmila-dãe šud? Tamãm mardum nīz az în vāqî́a āgăh šudand, bi-ḥairal firo raftand. Yak-roz yakí az ān baccahã pas az copān-xāna murăja at kard. Yak-roz pidar dïd ki abrhā daur-i sang ră girifta ast. Båd dìd ki az hamin abrhă yak gosfand-i safed bar-āmad u daurādaur-i sang gardiš mekunad. Bacca ba pidar-i xud qișşa mekunad ki hamān duxtar ră (hamšira-yi kūcikiš) dar miyān-i abr u hamān gosfand hamrāh-iš jimāe mekunad. Mutahajyir šuda bă xud guft: În ci msl (?).. hast ki gosfand hamrä-yi duxtar-i kūcik jimă $\bar{a}^{`}$ mekunad? Šāyad yak vāqi`a rux dihad. Cand roz ba'd šikam-i hamān duxlar kalān šud, u hạmilagïyi vai xübtar zăhir gardỉd $u$ inak ba dỉbā (zãygãh) barā-yi zāidan meravad. Dar dïbū duxtar yak țifl bi-vujūd ăvurd. Aqāinib barä-yi tabarrukì ba dībü raftand. Duxtar vaqtï-ki aqărib-i xud rä dìd. . . ţifl-i nauzăda-yi xud ră pinhăn kard. Birădar-i xurd ba dỉbü raft, u guft: Cūn man hameša tură ba pušt megaštāndam, mardum taşavvur mekunand ki man hamrä-yi tü jima karda am, u marã badnām sāxta-ĩ. Hạāā ki man badnām šuda am, u hama ba man bohtãn (U.T.) mekunand, cị̣aur ṭ̂ll-i nauzāda-yi xud rã az man pinhăn mekuni? Duxtar dar javāb-i birādar guft ki: Bălä nigăh kun! U vaqtī ki birădar-iš ba āsmān nigäh kard, asb i surxi dĩd ki hamān ṭifl-i nauzāda-i xwāhariš bālă-yi ăn suvār ast. U rūy-i ān țifl ānq̣adr mušcal u raušan būd ki ba yak nigāh rŭy-i bacea rā soxtānd. U ba‘d ba zamin uftād. Be-hoš gardīd. Ba‘d ba jān āmad u xud ră clar dih-kada ba xãna-i xud yāft. Sĩ roz-i duxtar dar dībü ba elmăm (U.'T.) rasĩd. U ba xăna āmad. Har do dast-iš cirā $\gamma$-cob u dar pušt-iš fifl-i nauzād-iš būd. Cirā $\gamma$-cob-i yak dast-i xud rā ba xāna guzâăšt, u hamrā-yi cirā $\gamma$-cob i dīgar az xāna xārij šud, u dar yak zaminn-i buland darūn šud. U ba'd pas bar āmad. Dar augrija darün šud, pas az augrija xärij šud. Dar în asnā pidar-iš rasĩd u duxtar ră ta'qïb kard. Duxtar david u pidar ba dunbāl-i vai. Andaki ba`d duxtar dar yak hạau, hamrā-yi ţifl-i nauzăd-i xud dar āmad. Pidar nīz dar hạaų dar āmad. Ammā ba‘d dīd ki dar hạuz hec äb nest, u

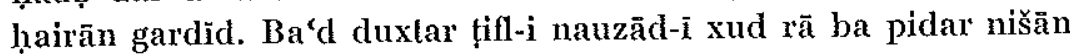
dād. Ţifl-i nauzād ba pidar-kalān guft ki man Xudāy-i šumā hastam. Ba`d pidar, duxtar u hamān tifl-ĩ nauzãd ba-xāna murāja‘at kardand, u sih sāl ba manzil būdand. (Duxtar, pidar u țifl-i nauzād dar hauž bāqī māndand. Duxtar ba pidar guft ki : 
Ciqadr vaqt ast ki injā hastĩ? Guft: Sih šab. Duxtar guft: Na, sih sãl ast ki dar in hauẓ hastĩm). Duxtar ba pidar guft: Ḥālä bu-rau, u peš az ãnki mã rā tark güī, az in ţifl pìähan-iš rā xwāhiš kun tã ba-qiism tụ̣fa ba-xãna ba-barì. Pidar pirāhan-i țifl rā az nazd-î̌ darxwãst kard. Yak šab guzašt u subh țifl-i nauzãd ba pidar"kalăn megüyad: Aknün ki šumã meravĩd, ci tụ̂fa-i ba šumā bi-diham? Pidar-kalān guft: Man az tũ juz pīrāhan-i xudat digar cìzi na mexvāham. Țiff pīrāhan-i xud rā ba pidarkalān dāe u vai ba xāna bar-gašt. Šab hamān pīrāhan rā hamrā-yi zan-i pìr-i xud yakjā pošida, xvāb kardand. Subḥ ki az xvāb bar-xvāstand, har do-ī̌sãn juvān šuda būiand.

\section{Translation from Persian by Professor Georg Morgenstierne:}

For this Kuzum ${ }^{51}$ one wife had borne seven sons and one daughter. And he sent those seven sons and the one daughter to the bända ${ }^{52}$ And they settled at the bạnda. News arrived that the small daughter had become pregnant. Her father was astonished: "How has the small girl become pregnant?" All the people also became aware of this event and they became astonished. One day one of those boys returned back from the banḍ. One day the father saw that clouds had gathered round the stone. Then he saw that a white $\mathrm{ram}^{53}$ came out of these clouds and circumambulated the stone. The boy told his father that that ram is copulating with the girl (his small sister) inside the cloud. He became perplexed and said to himself: "What wonder(?) is it that a ram is copulating with a little girl? It may be that some (strange) event will take place."

s1 I have: Are(k) Kuzum had seven sons and one daughter from one wife.

52 băṇa (Pashto) = shepherds' shelter on a mountain pasture, saeter.

s3 Georg Morgenstieme in private communication (20th of Feb. 1957) writes, that he has in his dlary from the Avicenna festival in Hamadan the following information from Mr. Breshna, the Afghan painter and cultural at taché in Teheran: "In Kamdesh a male goat is locked up for three winter months and is released at Nauruz. A young and beautiful girl in bridal dress is sent to meet it. If the male goat speeks to her, the year will become fine. If it is frightened and runs from her up into the mountains, the year will become dry".

The informants of Mr. Breshna were several persons in Kamdesh and moreover General Safar Wakil Gharzat and the wife of Shah Mahmud Khan, both ont of Nuristani families. 
Some days later the belly of that girl grew big and her pregnancy became more evident and she goes to the birth-house ${ }^{54}$ for the delivery. In the birth-house the girl brought forth a child. Her relations went to the birth-house to congratulate her. When the girl saw the relations ... she hid her newborn babe. Her brother went to the birth-house and said: "Since I always used to carry you round on my back (ef. fig. 28), people imagine that I have cohabited with you, and you have given me a bad reputation. Now when I have got a bad reputation, and they all run me down (U.T.), how (is it that) you are hiding your newborn babe from me?" The girl answered her brother: "Look upl" And when her brother looked at the sky he saw a red horse, and his sister's newborn child was riding on it. And the child's face was so bright and shining that it burnt the boy's face when he looked at it once. Afterwards he fell to the ground. He fainted. When he came to his senses again he found himself in the village, in his own house.

The girl's 30 days in the birth-house came to an end. And she came home. In both hands she held torches and on her back she carried her newborn babe. She left the torch in one of her hands in the house and she went out of the house with the other torch, and she went up into the highlands ("a high ground"). And afterwards she came up(!) again. She entered the hole, and again went out of it. In the meantime her father arrived and followed her. The girl ran, and her father after her. After a little while the girl plunged into a lake ${ }^{55}$ logether with her newborn babe. Her father did the same. But then he saw that there was no water in the lake and he got astonished. Then the girl showed her newborn babe to her father, 'The babe said to its grandfather: "I am your God." Then the father, the daughter and the babe returned home and stayed there for three years. (The girl, the father and the babe remained in the lake. The girl said to her father: "How long time have you been here?" He said: "Three nights." The girl said: "No, we have been in this lake for three years.") The girl said to her father: "Now go away, but before you leave me,

54 Paruni: dibu (cf. map).

${ }^{5 s} \mathrm{Cf}$. footnote 29, and Rob. 1896 p. 383 and Morgenstierne 1951 p. 168 about the birth of Bagisht. 
ask the child to give you its shirt, in order that you may carry it home as a gift." Her father made a request from the child for its shirt. One night passed and at dawn the babe says to its grandfather: "Now when you are going, what gift shall I give you?" The grandfather said: "I want your shirt and nothing else." The child gave him its shirt, and he returned home. At night he put on the shirt logether with his old wife, and they went to sleep. At dawn, when they rose from sleep, they both had become young again.

Informant: Zãmān.

No. $11 \mathrm{~B}$.

Locality: Shtiwe.

Date: 17 th of July 1949 .

Kusum and his wife were old. They had six sons and one daughter. The daughter and one of the sons went together to the cattle shed of the mountain pasture.

The daughter became pregnant.

The other brothers went with swords to kill their brother, but the sword could not cut. The daughter fled to a plateau with red clouds. The brothers followed and saw white goats. They understood that she had conceived by one of them.

Later she gave birth in a separate place and after much trouble she showed her child.

Eighteen colours radiated from the child. 'The brothers, who saw this, went home and told their father Kusum about it.

Kusum followed the daughter to a cave, from there to a lake. There was a sun in the lake. They went into the lake and met the god by whom she had conceived. The god gave Kusum a shirt. Clad in this shirt he slept together with his wife. Next morning they had become young again.

The daughter stayed with the god.

Kusum was the ruler of all the other Paruni. The house of Kusum was the god's house. The god had his separate place in the house (cf. fig. 21). 
Informant: not stated.

No. 11 C.

Notation: (in Persian) Ahmad Ali Motamedi (diary p. 86).

Translation: Moh. Karim Nushin.

Locality: Shtive.

Date: October 1953.

A thousand years having passed since Creation Mära said: "I will go to the Earth and make relationship with the people there." And thus it happened that he came to the Earth on green, red, and blue clouds.

He cohabited with a virgin by name Simsuga who was going alone on the green slopes of the mountains.

As a result of their copulation she gave birth to a son by name Himindu.

And Māra said that when worshipping, people should invoke his name and when praying, people should remember Suga and Himindu.

Informant: Zāmān.

$$
\text { No. } 11 \mathrm{D} \text {. }
$$

Notation: Klaus Ferdinand (diary III, 35).

Locality: Shtiwe.

Date: October 1953.

Interpreter: Ahmad Ali Motamedi.

A thousand years ago Mara, who equals Hafiz, came to the Earth and slept with a girl, So'gäd, in the open land. 'They got a son together. His name is Hemondo.

Māra said: "People must remember me when they worship, and when they pray they must remember my son and my wife."

No. 12 .

Informant: Zămān.

Locality: Shtiwe.

Date: 17 th July 1949 .

The people of Parun were attacked by the other Nuristanis and by the Minjanis and were dispersed. 
They were assembled again by a flute-player in Parun. Sixty persons were assembled who werc the ancestors of the Paruni of today.

Informant: Zămān.

No. 13.

Locality: Shtiwe.

Date: 17 th July 1949.

There was a lake with seven lights. A man went there and hit the lights [with a stick?].

From one of the lights came the golden stool ${ }^{56}$ which later came to Badakshan.

No. 14.

Informant: Abcul Aziz(?), Pošuk-tadba.

Locality: Urtā above Shtive.

Date: 1 st of August 1964.

Tape-recording: 1964, no. 86 (informant cxplains himself in Persian).

Free rendering:

The gods (lūžū) gathered on this side [of the river] and the giants on the other side. Both of them began to play. The game that was played by the giants was better than the game that was played by the gods. Then the gods talked together, discussed, and said to themselves: "Our play was not good. Why not?"

There was a woman who was called Živud. She was sent to that mountain [informant points at the mountain] to find one of the gods who was not present at the game. "If she finds that god then our play will succeed!"

She went and went until she reached the top of a high hill. She looked at the lake [up there] which was moving.

'The water was moving and in the water she(?) came out as a calf $[=$ in the water a calf appeared $],{ }^{57}$

se Cf. foolnote 29 , and Rob. 1896 p. 387 , story 7.

53 The Nuristani seem to be most fascinated by lakes, especially when the surface of the lake is moved by the wind. On the background for this cf. Buddruss 
The calf had a flame [which came] out of its mouth and that flame burned some parls (Persian: parahaiš) of Žiwud. Since she was burnt, she was not able to fly and had to sit there. The calf disappeared into the water again and emerged after a while and threw the water, [so that] the water touched the wings of Živud. 'This caused the wings to be repaired again.

Then Živud told the calf: "Come after mel" When the calf had followed her for only a few steps it became bigger. By each step the calf grew bigger and bigger until it caught up with Žiwud, when it was a big $o x$.

Then the calf gave the hill a push with its two big horns. Živud was frightened and ran away from the ox and hid herself under that stone-that stone in front of us. Ziwud entered there, and the gods who saw that strange creature were afraid. Then Mănde came to them and said to them: "Don't go away from me. I am from your elan. ${ }^{58}$,

Then they gathered again(?) and this time the play of the gods was much better than that of the giants, and the giants' play was bad and the giants were defeated. ${ }^{69}$

And this was the story.

No. 15 .

Informant: Wäci, ${ }^{60}$ Wäči- or Disni-ladba.

Localily: Shtiwe.

Date: October 1953.

Tape-recording: Shtiwe 8.

Wäci was probably the head of the Disni clan. About the temple to the goddess Disni and her statue KK 11 A cf. Motamedi, A.A. \& Lennart Edelberg 1968.

Wäci was brought to the guest house on our request for somebody to play the four-stringed guitar (urb'ã). He came staggering

1960 p. 204. Personally I think that the reflected jmage of the sun from the stirface of a lake is the background for many of these Nuristani ldeas (ct. footnote 29. About the calf see also footnote 29 ).

s8 Informant used the Kati word "dere" (Partni: tadba = clan, sib (lineage).

so Cf. Buddruss 1960 p. 204.

60 Acc. to G.B. the male name 'Wäci' has probably no commection with 'wäè' = fairy. 
in and there inside the room he played and hummed the five hymns no. 15-19 (fig. 29).

Wäči could only speak Paruni. I suppose Zämān's comment to me in 1948 alluded to him: that there were still some Kafirs in Shtiwe. They were respected on account of their honesty and good qualities-but they were teased for their Kafir faith-and their sons were Muslims. They said, however, to the young people: "You console yourselves with beholding God in the life to come, but we prefer to see him in this life" (Lennart Eclelberg 1952).

W iči seemed to be very intelligent, but his mind was somehow not present while he performed his hymus. Klaus Ferdinand murmured: "This is the most powerful I have ever witnessed!" (Lemuart Edelberg 1956).

When Wäči later heard his own voice through our tape-recorder he said that he wished he still had the voice of his youth.

Vocal solo accompanied by ' $u b^{\prime} \bar{a}^{\prime}$ (the ' $u \mathrm{r}^{\mathrm{b}} \mathrm{a}$ ' is a fourstringed guitar played with a plectrum $\left.{ }^{6 \mathrm{t}}\right)$.

Translation into Persian by the surrounding persons and noted by Ahmad Ali Motamedi :

$$
\begin{aligned}
& \text { مسرود قلديم ششوبو }
\end{aligned}
$$

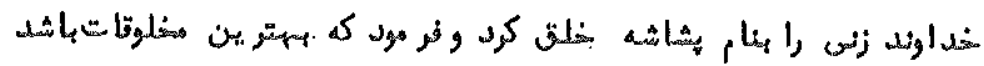

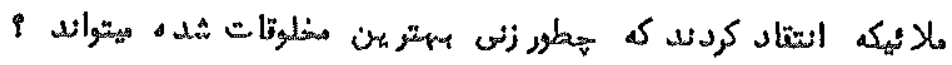

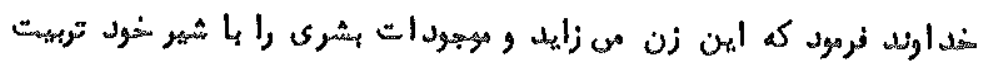

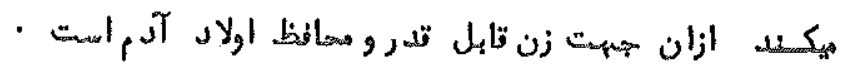

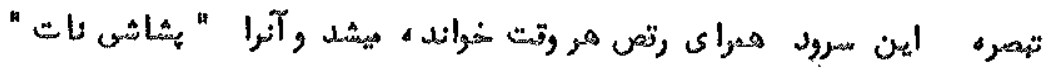

$$
\begin{aligned}
& \text { - } 1.50
\end{aligned}
$$

61 Instruments of this and other kinds from the Parun Valley are found in the Ethnographical Collections of the National Museum (now at Moesgard/University of Aarhus).

The 'urba' was not tuned up (Paruni: arcäi cuink) for no. 15. A younger man tuned il later (cf. tape recording). 
'Transcription from Persian by Professor Georg Morgenstierne:

Surōd-i qadim-i Š̄ūpū.

Xudāwand zanī rā ba năm.i Pušăši xalq kard, u farmŭd ki bihtarīn-i maxlūqāt băšad.

Malä’ika intigāa kardand ki ěi-țaur zanì bihtarīn-i maxlūquăt šuda mētuwānad?

Xudäwand farmŭd ki in zan mēzāyad u maujŭuăt-i bašarī rā bã šî̀-i xud tarbiya mēkunad.

Az ān jihat zan qābil-i qadr u muḷāfīzu-i aulād-i Ādam ast.

Tabșira :

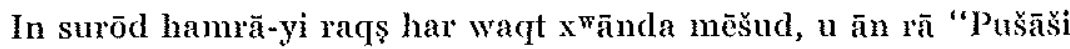
nāt" mēguftand.

'Translation from Persian by Moh. Karim Nushin:

The old song of Shupu. ${ }^{62}$

God created a woman named Pušāši ${ }^{63}$ and said that she was the best of all ereatures.

The angels criticized: How could a woman be the best of creatures?

God said, that this woman will bear a child and will develop all the humanity with her milk.

This is why woman (women?) is respected and the keeper of humanity.

Note:

'This song was sung with dances which were called "Poshāshinăt".

${ }_{62}$ Shupu = Shtiwe.

63 Cf. footnote 14. 
Informant: Wäči.

No. 16.

Locality: Shtiwe.

Date: October 1953.

Tape-recording: Shtiwe $12 .^{64}$

Performance: like no. 15.

Translation into Persian: like no. 15:

$$
\begin{aligned}
& \text { نام غئزل }
\end{aligned}
$$

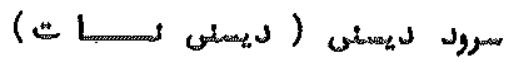

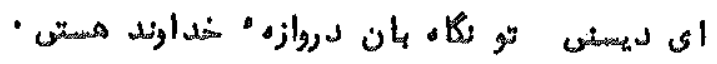

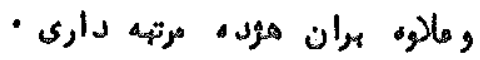

$$
\begin{aligned}
& \text { - مان } \\
& \text { - } \\
& \text { - ن }
\end{aligned}
$$

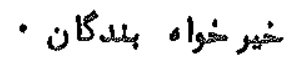

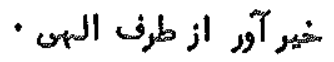

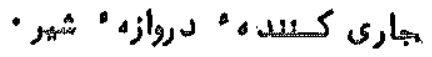

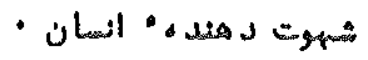

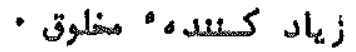

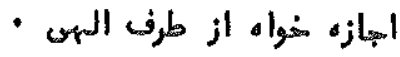

$$
\begin{aligned}
& \text { - تروازه }
\end{aligned}
$$

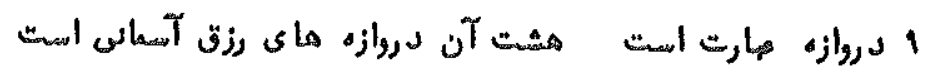

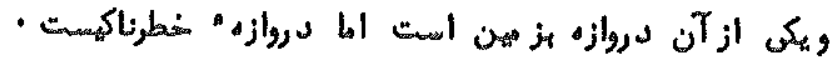

of This hymn has been published in Motamedi, Almad All \& Lemart Edelberg 1968: A Kafir Goddess. 
Transcription from Persian by Professor Georg Morgenstierne:

Näm-i Yazal: Mënōntarī.

Surōd-i Dĩsnì (Dìsnī Năt).

Ai Dīsnī. Tu nigāhbān-i darwăza-yi Xudāwand hasti. U 'alāw(a) bar ān haždah martaba dārī.

Nigăhbān-i but-xāna. Š̆̌r-clihanda-yi mauǰŭdāt-i bašarĭ. Nigăhbăn tawallud šudagăn. Xair- ${ }^{w a ̆ h}-i$ bandagān. Xairẵvar az țaraf-i Ilahī. Jãrĩ kunanda-yi darwãza-yi šĩ. Šahwat dihanda-yi

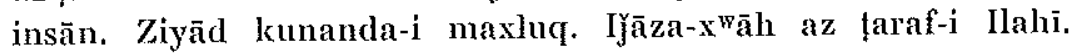
Nigāhbān-i darwãza-yi rạ̣mat.

Darwāza 'ibārat ast: Hašt ăn darwāzahānyi rizq-i āsmānī ast. U yakī az ān darwāza ba-zamin ast. Ammā darwãza-yi xaţarnākīst.

Tabşira: In surōd maxşūṣ-i Šūpū ast u ba dihkada-yi Prōns u Dîwa táalluc na dārad. Dar māh-i Nīlö (arvwal-i bahār) bară-yi yak hafta hamrä-yi atan (dance? Pashto: atạ̣?) in suröd xwānda mëšawad. Ma'ārif ba-rūzhāīst ki ramahā az dihkada

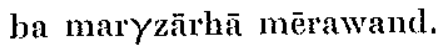

Translation from Persian by Professor Jes Asmussen:

"Oh, Disni, you are the protector of the gates of God and moreover you have eighteen grades:

Keeper of the temple,

Giver of milk to human beings,

Protector of infants,

Well-wisher of man-kind,

Bearer of wellfare from God,

You keep the door of milk flowing,

You bring sensuality to mankind,

You increase what is created,

You are the one who receives permits from God,

And you are the keeper of the nine gates of mercy." 
"The explanation of the nine gates is: Eight of them are the gates of heavenly riches, - and the ninth is on Earth, but is a dangerous gate".

\section{Note:}

This song is exclusively for Shtiwe and is not for the villages of Pronz and Dewa. It was sung in the month of Ni-la in the beginning of the spring. It is known in connection with the day when the flocks are taken from the village to the mountain pastures.

Informant: Wäči.

No. 17 .

Locality: Shtiwe.

Date: October 1953.

Tape-recording: Shtiwe 13.

Performance: like no. 15.

Translation into Persian: like no, 15.

$$
\begin{aligned}
& \text { نات مسارا } \\
& \text { ا ا }
\end{aligned}
$$

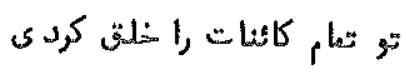

$$
\begin{aligned}
& \text { تو آل }
\end{aligned}
$$

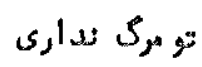

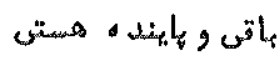

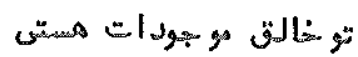

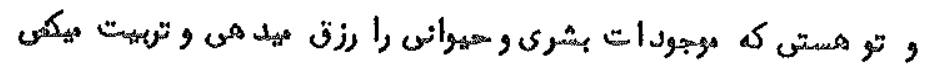

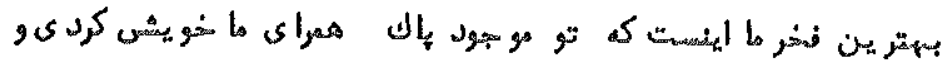

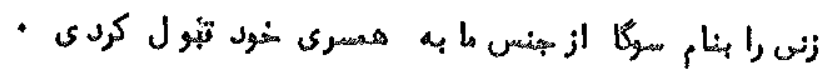


Transcription from Persian by Professor Georg Morgenstierne:

\section{Ai Mārā}

$$
\text { Nāt-i-Māră }
$$

Tu tamäm-i kāi-năt rã xalq kardī

Tu awval u axxir hasti

Tu marg na dāì

Bāciì u pāyanda hastĩ

Tu xãliç-i mauy̆ūdāt hastĩ

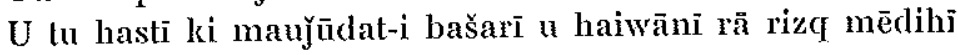

u tarbĭyat mēkuni

Bihtarĭn faxl'-i mã in ast ki tu maujŭd

hamrā-i mã xauli i kardì u zanī

ra ba-nām-i Suga az jins-i mã

ba hamsarì-yi xud qabul kardì

Translation from Persian by Moh. Karim Nushin:

Oh! Mãra

\section{Nāt-i-Māra.}

You have created all the worlds

You are the first and the last

You have no death

You are eternal and everlasting

You are the creator of all things

You are the one who feeds humans and animals and cause them to grow old

Our best pride is that you, who are a pure substance, came in relation with us and accepted a woman from our race (sex?) named Suga as your wife.

Informant: Wäči.

No. 18 .

Locality: Shliwe.

Date: October 1953.

Tape-recording: Shtive 14.

Performance: like no. 15.

Translation into Persian: like no. 15: 
I. lo $=i m \infty$

$$
\text { اي مارا }
$$

Transcription from Persian by Professor Georg Morgenstierne:

\section{Ai Mārā}

Dar șifat-i Mărã

Tū azalì büda-ĩ

Hìc kas na mēdănad asūs-i tu-rã

In butãn rā ki parastiš mēkunîm,

barä-yi ān ast ki ba-tu rasida na metuvānīm

U mēxvăhīm ba-vaș̣-i ānhã ba-tu bi-rasĩm

Pas dar haqiquat turā parastiš mekunīm, na butān xã

Translation from Persian by Moh. Karim Nushin:

Oh! Mara

$$
\text { In the praise of } \text { Mara }^{85}
$$

You are the Eternal

Nobody knows your foundations (basis, realities)

We worship these statues, because we cannot reach you

And we wish we can reach you by means of them

And so in reality we worship you and not the statues.

65 Wä̀'s favorite song. In Kafir times it was performed at any time together with a dance.

6 Acta Orientalia, $\mathrm{XXXIV}$ 
Informant: Wäči.

No. 19 .

Locality: Shtiwe.

Date: October 1953.

Tape-recording: Shtive 15.

Performance: like no. 15.

Translation into Persian: like no. 15.

$$
\begin{aligned}
& \text { ن } \\
& \text { اى sان }
\end{aligned}
$$

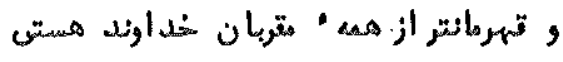

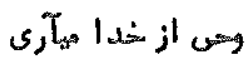

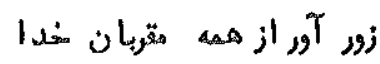

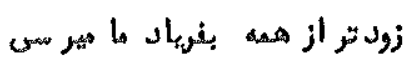

$$
\begin{aligned}
& \text { و بو قت هصيهيت بتو رسهيل ه هيتوانيم } \\
& \text { وتو شاهنهار و بالدار هستين }
\end{aligned}
$$

Transcription from Persian by Professor Georg Morgenstierne:

Dar şĩfat-ĩ Mān

Ai Mān

Tu mudāfía-i ma'îbal hastĩ

'Tu qahra män tar az hama muqarrabān-i Xudāvand haslì

Vahy az Xudā mēāri

Zör-āvar az-hama muqarrabān-i Xudā

Zūd tar az hama ba faryăd-i mä mērasĩ

Ba vaqt-i ma ibat ba tu rasîda mētuvānĩm

U tu šaxdār u bāldar hasti

Translation from Persian by Moh. Karim Nushin:

In the praise of Măn

Mān-You are the defender of Distress

You are the hero of courtiers of God 
You are bringing revelations from God

You are the mightiest of all courtiers of God

You are arriving soon to our calls

And in the time of distress we can reach to You

And You have horns and wings.

No. 20.

Informant: A man from Shtive.

Locality: Kunjeni pass.

Date: 2nd of August 1964.

Tape-recording: $1964,88 \& 89$.

Free rendering:

There is a man: Mānd $\bar{e}^{66}$

He has a kind of rope

He has brought this rope ${ }^{67}$ from the sky

He has taken the rope and given it to Giwiš

Giwiš in turn has given it to all the chiefs

And by that like a magic-like a telephone-he could inform them of all the news.

No, 21 .

Informant: A man from Shtiwe.

Locality: Kunjeni pass.

Date: 2nd of August 1964.

Tape-recording: $1964,87 \& 89$.

Free rendering:

Diwog $^{88}$ lives in the mountain in this place

He has lots of cattle

Equally lots of ghee

He has taken part in the fighting between Kafirs and Muslims

He has killed lots of people

$\mathrm{He}$ is a brave man.

\footnotetext{
of Cf. Morgenstierne 1951 p. 164.

${ }^{67} \mathrm{Cf}$. Morgenstierne 1951 p. 168-174 (the Ushe-Rope).

${ }^{8} \mathrm{Cf}$. Snoy 1962 p. 133 and 141.
} 
No. 22.

Informant: A man from Shtive.

Locality: Kunjeni pass.

Date: 2nd of August 1964 .

Tape-recording: 1964, 90 \& 91.

Free rendering:

It was a woman

Her name is $\operatorname{Kim}^{1} \mathrm{a}^{69}$

She was a woman, but like a man

She goes into the lake

She crosses it

And comes out of it.

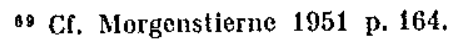


Pashki:

IV. Calendars of Parun.

Infomant: Abdur Rahim (Istan-tadba) and others.

Date: 20 th of July $1949=17$ th of Mun-lau.

"Month" Days Comments 1949 and 1953

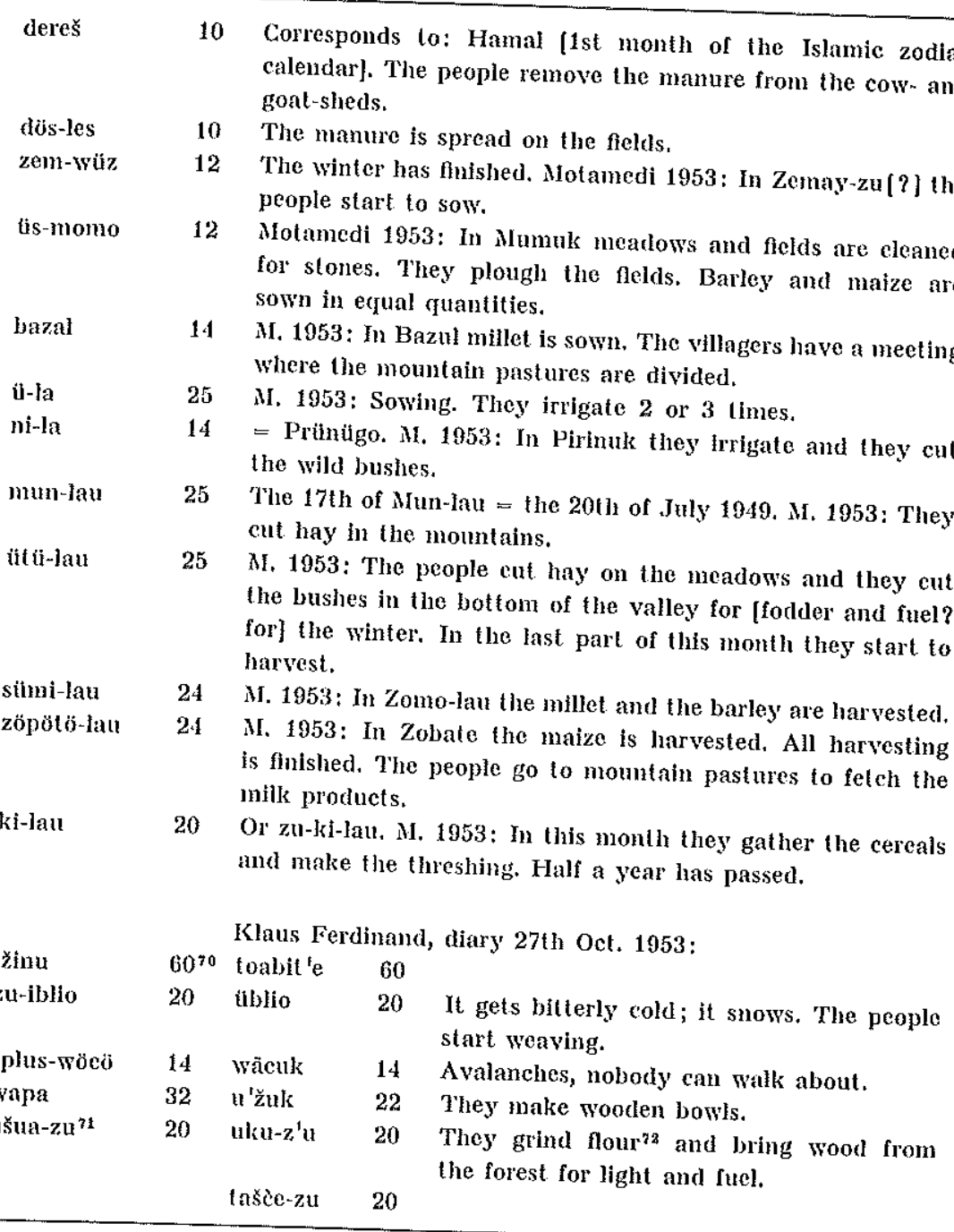

Notes see p. 84 . 
Kushleki I:

Informant: Jan Gul (fig. 30 right) (cf. Kushteki II).

Date: 18 th of July 1949 .

\begin{tabular}{|c|c|c|c|c|c|}
\hline "Period" & Days & Comments & "Month" & Days & Comments 1949 \\
\hline \multirow[t]{3}{*}{ ustäls } & \multirow[t]{3}{*}{33} & \multirow[t]{3}{*}{ Snow \& rain } & dros & 12 & Rain is coming. \\
\hline & & & numuk & 12 & $\begin{array}{l}\text { The grass comes up and } \\
\text { the flocks will eat it. }\end{array}$ \\
\hline & & & samušuk & 12 & $\begin{array}{l}\text { Goats and sheep can be } \\
\text { milked. }\end{array}$ \\
\hline \multirow[t]{7}{*}{ sumbara } & \multirow[t]{7}{*}{$147^{73}$} & \multirow[t]{7}{*}{ Happy days } & nila-lau & 24 & $\begin{array}{l}\text { Milk-products (Persian: } \\
\text { sirwar) are brought from } \\
\text { the mountain pastures. }\end{array}$ \\
\hline & & & mul-lau & 18 & The meadows are cut. \\
\hline & & & wiški-lau & 24 & $\begin{array}{l}\text { People go to the moun- } \\
\text { tains and cut grass for } \\
\text { fodder. }\end{array}$ \\
\hline & & & sumi-lau & 24 & The wool is cut. \\
\hline & & & zapato-lau & 24 & $\begin{array}{l}\text { The wheat and the barley } \\
\text { are ripe. }\end{array}$ \\
\hline & & & kil-lau & 20 & "Chcese". \\
\hline & & & wac-twa & 12 & Walnuts etc. are collecled. \\
\hline \multirow[t]{2}{*}{ izmara } & \multirow[t]{2}{*}{180} & \multirow[t]{2}{*}{$\begin{array}{l}\text { Winter, snow } \\
\text { is coming, } \\
\text { flour is brought } \\
\text { to the mill. }\end{array}$} & sires̆ & 60 & $\begin{array}{l}\text { The arrival of the winter. } \\
\text { The flocks are brought } \\
\text { from the mountain pasture } \\
\text { to the village. }\end{array}$ \\
\hline & & & lznara & 120 & $\begin{array}{l}\text { The arrival of the snow } \\
\text { and the coldness. People } \\
\text { take on boots (Parumi: } \\
\text { cantus) and two cloaks } \\
\text { and mittens. }\end{array}$ \\
\hline
\end{tabular}

70 M. 1953: The 4th of Aqrab which is the same as the 1st of Okozo(?) the people start in the morning for the mountain pastures. Before noon they return with whey, which they drink.

$" 1$ išua-zu and dereš together are called žobözll.

72 Thls information sounds peculiar; it may be that the streams are frozen so that the water-mills cannot function and the people must grind flom with hand-mills.

7s ustäiš and sumbara $=180$ days (Persian: no bist).

3 In Parun the men wear a short woollen cloak in summer and a long wollen cloak in winter; when it is very cold they may wear both. 
Kushleki II:

Informant: Palyuk, "99 years old" (cf. fig. 10 tomb), helped by his son.

Date: $19 t$ li of July 1949.

Genealogy: Gander

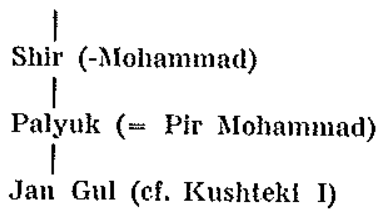

"Month" Days Comments

\begin{tabular}{|c|c|c|}
\hline dros & 10 & $\begin{array}{l}\text { Dros is also called "deres". In this month people from Kam. } \\
\text { desh and Kantiwo will come and sacriftce to god. }\end{array}$ \\
\hline dest-lis & 10 & \\
\hline mumuk & 12 & \\
\hline bazal & 12 & $\begin{array}{l}\text { On the first day of bazal there are sacriflces to the god. A } \\
\text { rich man will bring } 2-3 \text { goats. }\end{array}$ \\
\hline droskwa & 14 & $\begin{array}{l}\text { The flocks eat and we get the products, and after that we } \\
\text { bring cheese to the big god. We are living in the catlle sheds } \\
\text { near the village. }\end{array}$ \\
\hline$\ddot{\mathrm{u}}-\mathrm{la}$ & 14 & \\
\hline nü-la & 24 & $\begin{array}{l}\text { On the first day of nit-la they dance }{ }^{75} \text { Iprobably in the big } \\
\text { temple] and bring cheese [probably for sacriflce]. }\end{array}$ \\
\hline mul-la & 18 & \\
\hline wiskt-la & 24 & \\
\hline stumi-la & 24 & \\
\hline $\begin{array}{l}\text { zapötö } \\
\text { kil-la }\end{array}$ & $\begin{array}{l}24 \\
20\end{array}$ & \\
\hline $\begin{array}{l}\text { kil-la } \\
\text { wizi-wac }\end{array}$ & 12 & (The period from droš to wizi-wac is the summer). \\
\hline $\begin{array}{l}\text { wizi-wac } \\
\text { to'abitö }\end{array}$ & 60 & The grass has flished, the flocks and herds are eating leaves. \\
\hline $\begin{array}{l}\text { to'abitö } \\
\text { itplio }\end{array}$ & 20 & $\begin{array}{l}\text { On the last day of iplio people go from Pashki to Shtiwe } \\
\text { to dance and thereafter back to Kushteki. }\end{array}$ \\
\hline wacek & 12 & The cold increases. \\
\hline Žičik & 7 & They eat nuts [probably hazel nuts and walnuts]. \\
\hline opozo & 10 & They sacrifice to the dead. \\
\hline wafa & 18 & $\begin{array}{l}\text { They eat "mäst" } 78 \text { and bread. No festival. (The period from } \\
\text { to'abito to wafa is the winter). }\end{array}$ \\
\hline užum-usto & 10 & $\begin{array}{l}\text { On the last day of uzum-usto they sacriflce a goat to the big } \\
\text { god (Mareira). }\end{array}$ \\
\hline üršwa & 5 & On the last day of ürswa they sacrifice. \\
\hline disni-suto & 5 & $\begin{array}{l}\text { During all flve days of disni-suto they bring bread and cheese } \\
\text { to the temple. }\end{array}$ \\
\hline
\end{tabular}

Notes see p. 86 . 
Dewa:

Informant: not stated.

Date: 23 rd of October $1953=18 \mathrm{th}$ of $\mathrm{Ki}-\mathrm{la}$.

"Month" Days Comments

\begin{tabular}{|c|c|c|}
\hline mumuk & 12 & The field work starts. \\
\hline basāl & 12 & 'The animals are [still?] in the sheds near the village. \\
\hline wayầ & 5 & The flocks are leaving the village. \\
\hline ü-la & 20 & $\begin{array}{l}\text { The flocks are in the mountain pasture Tešatol (Tišatawod, } \\
\text { ef, map) for } 20 \text { days. }\end{array}$ \\
\hline zu-nülu & 20 & The flocks are in the mountain pasture of Tepes for 20 days. \\
\hline mum-la & 26 & The flocks are in the mountain pasture of Agok for 26 days. \\
\hline uški-la & 20 & People bring the bushes (for fire \& fuel) from the mountains. \\
\hline suminla & 23 & $\begin{array}{l}\text { The owner takes the wool. Wheat and food for animals [hay?] } \\
\text { are harvested. }\end{array}$ \\
\hline zobattä & 24 & The flocks come to the Tetot valley. \\
\hline ki-la & 23 & $\begin{array}{l}\text { When the threshing is fluished [probably the last day of ki-la] } \\
\text { a cow is killed by the mulla. }\end{array}$ \\
\hline wac-po & 12 & $\begin{array}{l}\text { The flocks are arriving to the village stables. The shepherd } \\
\text { [Paruni: u'za cf. Morg. } 1949 \text { p. } 279 \text { ] takes the wool. The } \\
\text { master takes on a new shepherd" }\end{array}$ \\
\hline toabit'e & 60 & Wood for fuel is collected from the mountains. \\
\hline üblu & 20 & \\
\hline wădsuk & 12 & \\
\hline upusu & 20 & The shepherd takes the wool. ${ }^{78}$ \\
\hline wap'a & 15 & \\
\hline wușum-ustû & 10 & $\begin{array}{l}\text { The master takes over the care of the anmals. In former } \\
\text { limes the inhabitants of Shtiwe came to Dewa in this month } \\
\text { to give a cow to be sacrifled to wuşum, the goddess of Dewa. } \\
\text { 'They sacriflced the cow by shooting an arrow into its neck. }\end{array}$ \\
\hline üršwa & 5 & New shepherds are employed ${ }^{7 \theta}$. \\
\hline dizni-sudu & 5 & \\
\hline dres & 10 & The shepherd takes the wool for the third time(?) \\
\hline das-liz. & 10 & \\
\hline$z \ddot{i} m a-\grave{i z}$ & 12 & \\
\hline
\end{tabular}

75 They had festlvals once in summer-in nila-la (nü-la) - and twice hn winter -in iplio and obor(?).

76 māst is Persian for 'coagulated sour milk'.

77 Acc. to my notes (ci. foot-note 79)).

78 If the shepherd during the winter gives food from his own store to the animals the milk and the wool belong to him.

70 Ace. to Almad Ali Motamedl's notes. 
Pronz:

Informant: not stated.

Date: 29 h of May $1954=3$ rd of sät-wayi.

"Month" Days Comments (if (1953) the comments are from 21 st of Octoler 1953)

$\begin{array}{lr}\text { üz-mumuk } & 12 \\ \text { u̇z-basal } & 12 \\ \text { sät-wayi } & 7 \\ & \\ \text { üngu-la } & 14 \\ & \\ \text { nuilö-la } & 23 \\ \text { mul-la } & 25 \\ \text { wiski-la } & 27 \\ \text { sumi-la } & 18 \\ & \\ \text { župate-la } & 24 \\ \text { ki-la } & 20\end{array}$

"To-day is the 3rd of sät-wayi because after 4 days the people will go to the mountain pastures".

4 The last day of each period is called "la" ("lau"). On that day they wlll come down to the village from the mountain pastures bringing the milk produets with them.

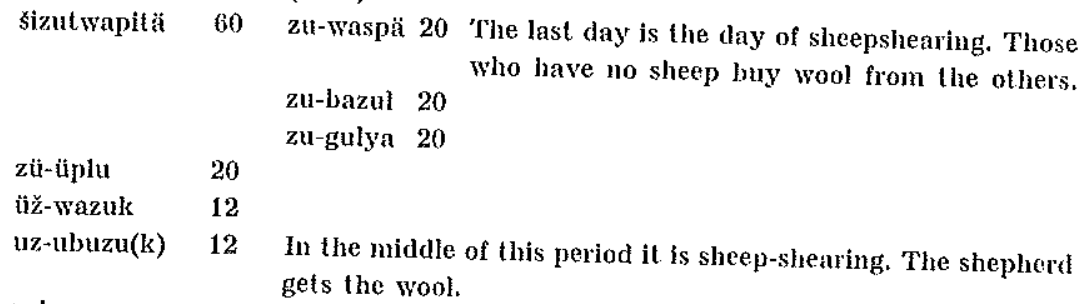

The last day is the day of sheep-shearing. The owner (master) gets the wool.

20 They have to fhish the threshing. The flocks and herds are coming down to the village and they start the feast in the temple (cf. fig. 32). They kill two goats, throw blood on the idol and divide the goat's meat. They sing special songs (1953).

\$ä

užumunustu 10

uč-uša $\quad 5$

disni-sulu 5

läs-d'res $\quad 10$

des-lis $\quad 10$

üz-sümuม $\quad 12$ 


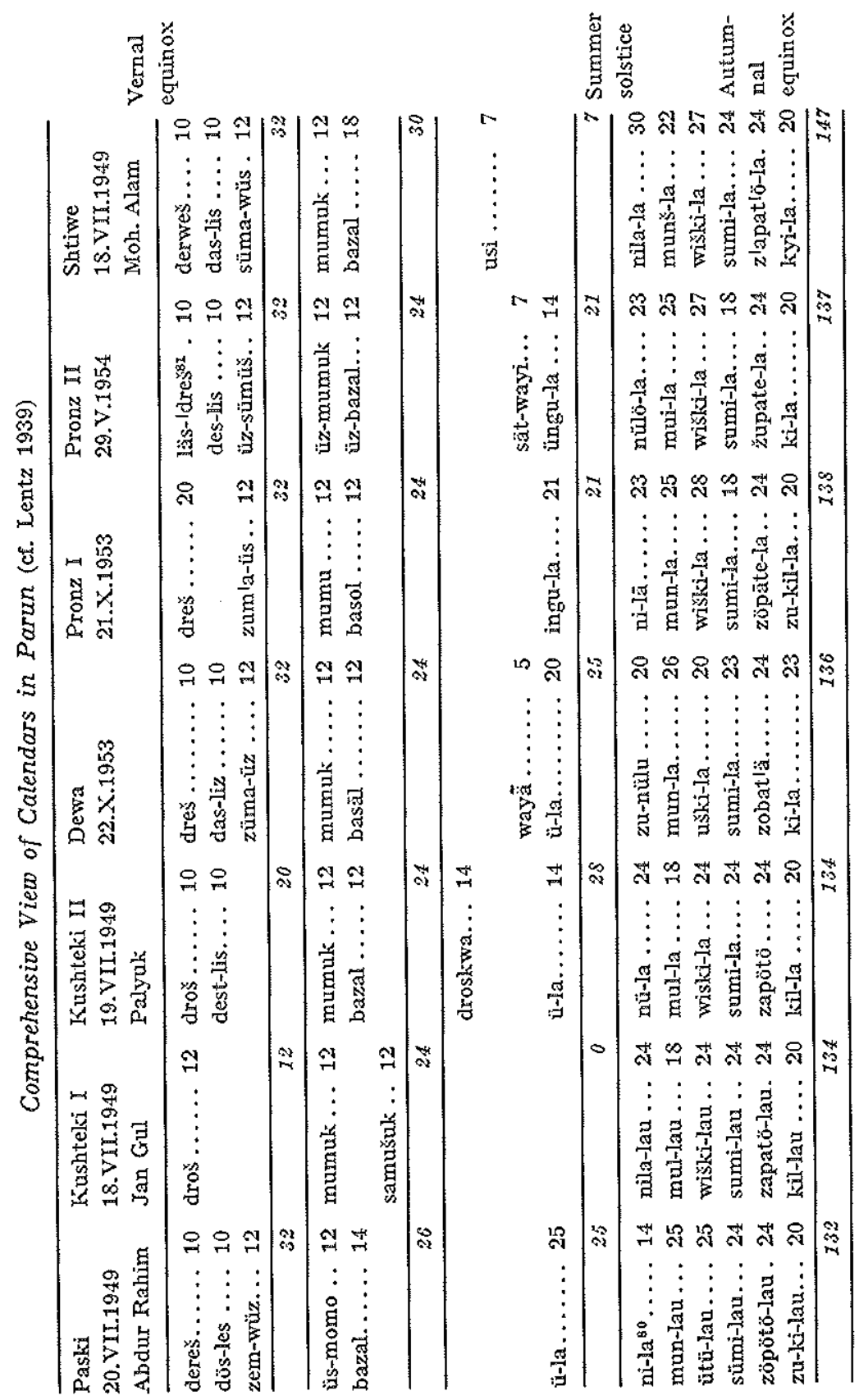


SONE PARUNI MYTHS AND HYMNS

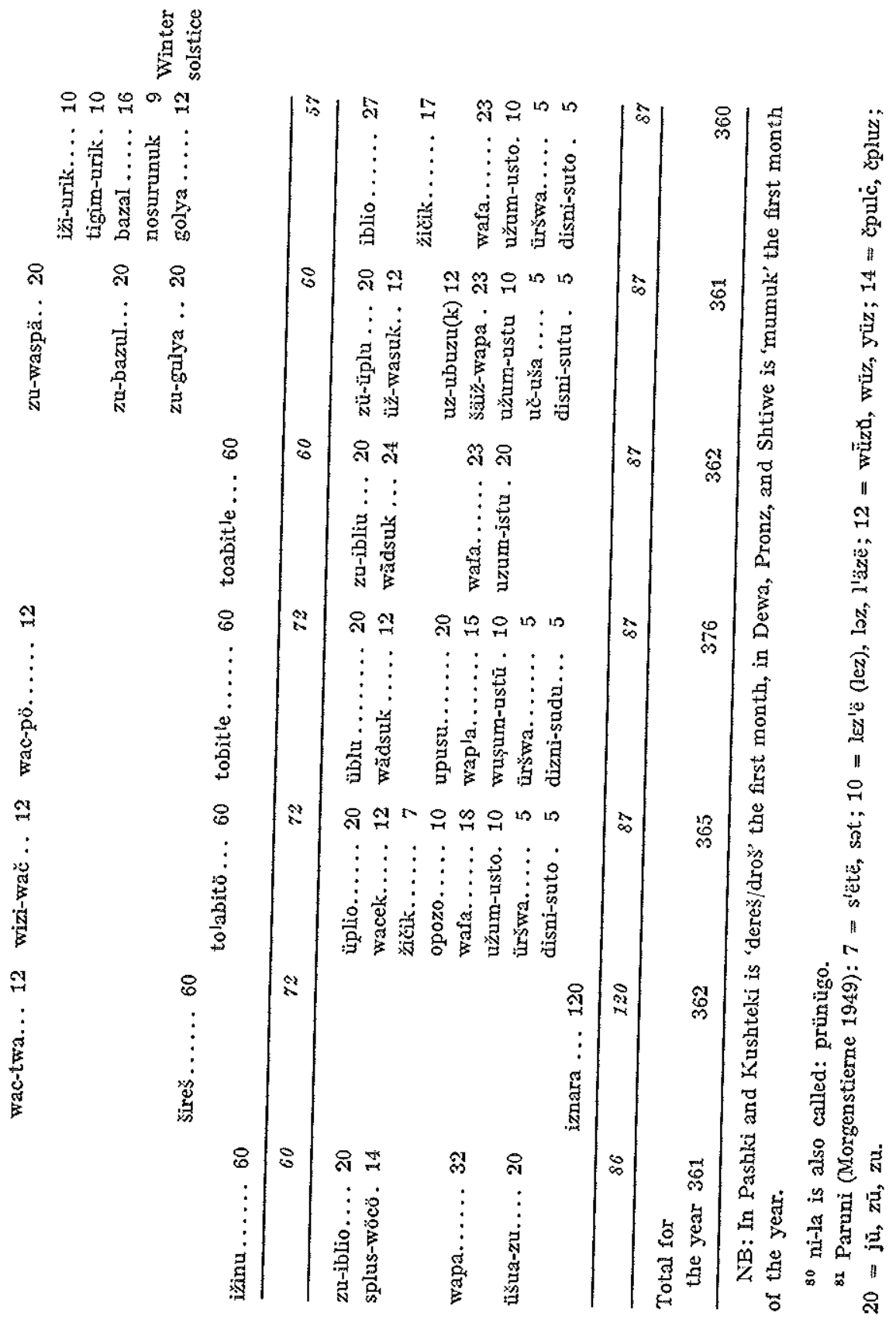


Shtiwe:

Informant: Molk. Alam (Pä'cog tadba).

Date: 18 th of Jtlly $1949=17$ th of munš-la.

"Month" Days Comments

\begin{tabular}{|c|c|c|}
\hline mumuk & 12 & Snow disappears. \\
\hline bazal & 18 & Flocks \& herds are satisfled. \\
\hline usi & 7 & Goats are owner-marked by euts. \\
\hline nila & 30 & The mountains lurn green. \\
\hline munšla & 22 & The middle of the season [probably the summer half]. \\
\hline wiškila & 27 & A thorny plant is cut for forlder [or fuel?]. \\
\hline sumila & 24 & "Sumi" means wool. They cut the wool. \\
\hline zapatelat & 24 & $\begin{array}{l}\text { Hay-making in the meadows. On the last day a cow was } \\
\text { sacrificed in the main temple of Marra in Kushteki. }\end{array}$ \\
\hline kyilla & 20 & Corn harvest. \\
\hline ižl-urik & 10 & $\begin{array}{l}\text { Means "new days" during which the flocks \& herds are } \\
\text { brought from the higher to the lower mountain pastures. }\end{array}$ \\
\hline tigin-urik & 10 & Flocks and heras are brought down to the village of Shtiwe. \\
\hline bazal & 16 & Snow falls. \\
\hline nosurunuk & 9 & $\begin{array}{l}\text { Saerifices for those who had died in the preceding time } \\
\text { [probably that summer]. }\end{array}$ \\
\hline golya & 12 & $\begin{array}{l}\text { "Gol" is the name of the valley. During these days those } \\
\text { who had a great authority walked down the valley spending } \\
\text { one night in each village. }\end{array}$ \\
\hline iblio & 27 & $\begin{array}{l}\text { After } 20 \text { days-when } 7 \text { days remained-the brave men, who had } \\
\text { killed Safi [i,e. Afghans], walked down to Pashki to dance. }\end{array}$ \\
\hline žicik & 17 & Menns fron. They threw an iron ball to show their strength. \\
\hline wafa & 23 & $\begin{array}{l}\text { The eight clans' houses, each possessing the image of a god, } \\
\text { gave bread to the other houses in Shtiwe. }\end{array}$ \\
\hline užutu-usto & 10 & $\begin{array}{l}\text { All the villagers went down to Dewa to honour the deity of } \\
\text { this village, Uzum [G. B.: Wuşum]. }\end{array}$ \\
\hline üršwa & 5 & $\begin{array}{l}\text { 'The poor [probably the shepherds] borrowed goats from the } \\
\text { rich [probably the owners], and after having cut the wool } \\
\text { they brought back the goats. }\end{array}$ \\
\hline disni-suto & 5 & $\begin{array}{l}\text { Sacrifices of goats to Disni, who was a goddess common to } \\
\text { all Kaffistan. If the goat shook itself, the sacriflce was ac- } \\
\text { cepted, if not, they lrad to bring another goat. }\end{array}$ \\
\hline derweš & 10 & Feast for the god Gis. \\
\hline daslìs & 10 & The weather gets mild. Therefore they sacrificed to all gods. \\
\hline süma- & 12 & Snow stops falling (süma $=$ snowfall). \\
\hline
\end{tabular}

82 I suggest that they may have walked down the valley to discuss with the experts in the lower villages whether their star-and/or sum-observations showed that the winter-solstice was near, so that finally-when they arrived at the chief temple in Kushtek-they could be fairly certain, that now it was the shortest day of the year. 
Pashki:

\section{Paruni Clans (tadba) ${ }^{83}$}

Istan $=$ Psni $($ Psnä, Pasni $)=$ Podmm $^{81} \ldots \ldots \ldots \ldots \ldots \ldots \ldots \ldots \ldots$ houses

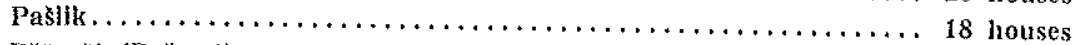

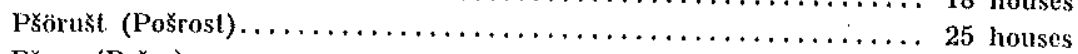

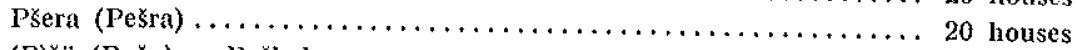

(P)šii $($ Pušu $)=\operatorname{Pošbak} \ldots \ldots \ldots \ldots \ldots \ldots \ldots \ldots \ldots \ldots \ldots \ldots \ldots, 20$ houses

Zikia ( Yema $^{85}$ i.e. the Siah Posh blacksmiths) .......... 4 houses

Zumu: ${ }^{86}$

Pubari (including Sapak) $\ldots \ldots \ldots \ldots \ldots \ldots \ldots \ldots \ldots \ldots \ldots \ldots \ldots$ houses

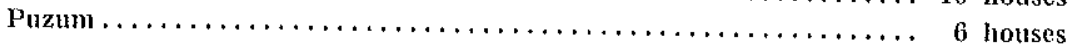

Kushteki:

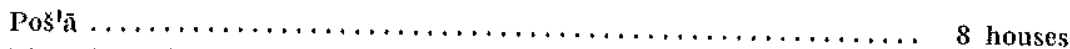

Pišta $($ Pošta $)=\operatorname{Tan}^{87} \ldots \ldots \ldots \ldots \ldots \ldots \ldots \ldots \ldots \ldots \ldots \ldots \ldots \ldots \ldots$ 15-16 houses

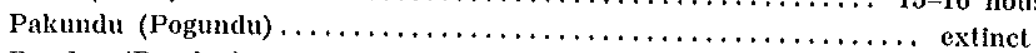

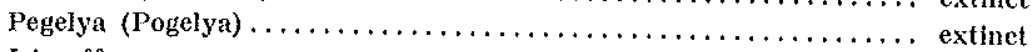

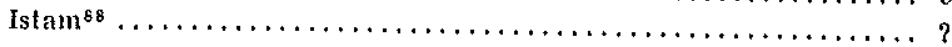

Dewa:

Zül $=$ Päzgum ${ }^{8 \theta}$.

24 houses

83 Deutsche' im Hindukusch 1937 p. 227 :

$\begin{array}{lllll}\text { Paschki: } & \text { Kuschteki: } & \text { Dewa: } & \text { Puruns: } & \text { Schtiue: } \\ \text { Ischtanderi } & \text { Pedschnlderi } & \text { Ischtanderi } & \text { IKascmderi } & \text { Kasemderi } \\ \text { Gregderi } & \text { Kulgurderl } & \text { Pasgemderi } & \text { Pomanderi } & \\ & \text { Mnerderi } & & \end{array}$

5i Malik Wali Mohammad and the wonderful artist and excellent informant Malik Abdur Rahim (cf. unprinted diartes 1948, 49, 53, 54, and 64, and Motamedi, A.A. and Lennart Edelberg 1968, Barrington 1960 and Buddruss 1960) both belonged to Istan-tadba. The malik is said always to come from this clan.

85 Morgenstferne 1949 p. 253 : iyom'a.

88 There are three water mills in Zimu, one for each clan.

87 The "Great Mulla of Nuristan" (cf. introduction) comes from this clan.

88 The Kusum- (cf. Shtiwe) and Istam- (= Istan?) tadba are said to be the ruling clans of Parun.

${ }^{89}$ Malik Nur Mohammad and Malik Bäz Mohammad belonged both to this clan. The day of sowing was always announced by Băz Mohammad from the mosque. He was according to Ahmad Ali Motamedi (diary p. 143) the most prominant man in Dewa, and according to Barringlon 1960: "... one of the holiest and richest men in the Parun valley". Barrington writes further: ". . They said he had 40 cows, more than anyone else in the valley. Mallk Nur Mohammad had $13 \ldots$..." 


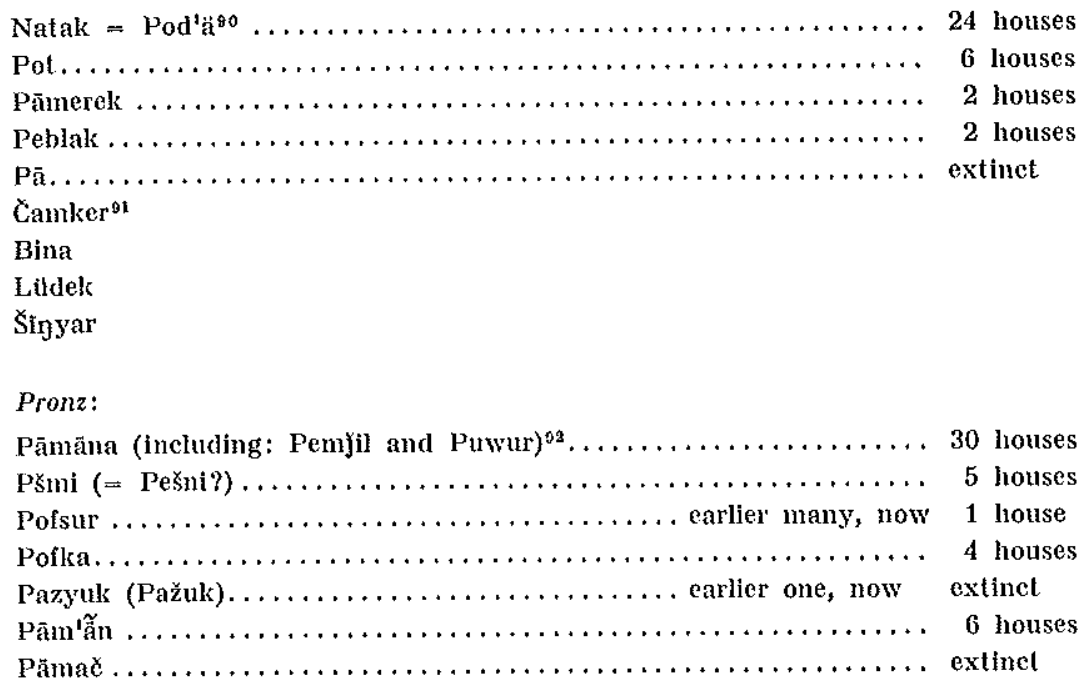

Shtiwe: ${ }^{83}$ (Total number of houses 80 )

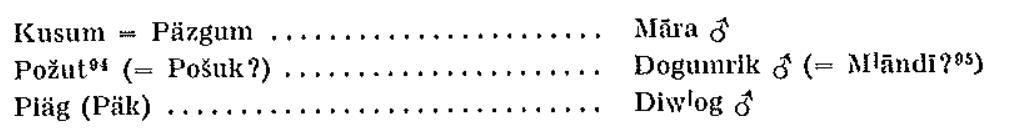

90 Malik Timur Shah belonged to Pocl'ä. His genealogy was:

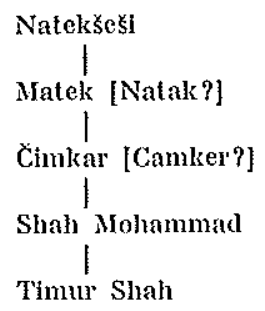

or It is likely that Camker, Bina, Liddek, and Šnyar are in some way identical with or included in Pot, Pāmerek, Peblak, or Pă. In connection with anthropometrical investigations $I$ know that at least 5 men belonged to Camker. But 1 do not know whether these 5 men had each his own house or household.

02 The malik comes from Paamāna, the ancestor of which was rich and courageous. Malik Amir Khan (1964) was, however, from Pstakok-tadba and $I$ do not know whether Pstakok is included in Pāmāna. A "hornstool" (șin-kñ) with carved designs on the backrest from his house is now in the Ethmographical Collections of Moesgård, University of Arhus.

${ }^{93}$ Cf. Motamedi, A.A, and Lennart Edelberg, 1968 p. 11. 


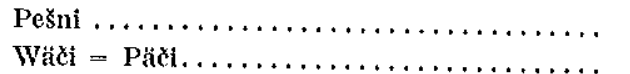

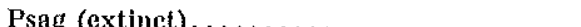

Pälčog (Pečik) $\ldots \ldots \ldots \ldots \ldots \ldots \ldots \ldots \ldots$ Jiwud (Žiwud) $o$ and Guj o

Poryiga (extinct) $\ldots \ldots \ldots \ldots \ldots \ldots \ldots \ldots$, Kimlä 우

Lapad ${ }^{\imath}, \ldots \ldots \ldots \ldots \ldots \ldots \ldots \ldots \ldots$ no idol (Persian: "but nadarad")

\section{Bibliography.}

Buddruss, Georg, 1960: Zur Mythologie der Prasum-Kafiren. Paideuma, Mitteilungen zur Kulturkunde, vol, 7, Heft 4/6. Wiesbaden.

Deutsche im Hindukusch, Anon. et al, 1937. Berlin.

Edelberg, Lemnart 1952: Træk af Landbrug og Livsform hos Bjergstammer i Hindukush. Næsgaardsbogen 1952. Nykobing Falster, Denmark.

Edelberg, Lemnart, 1956: Fra Kafirhytte til Ildtempel. Nasgaardsbogen 1956. Nykobing Falster, Denmark.

Edelberg, Lennart, Albert Schäfer, Volfgang Lentz, 1959: Imra, the Creator-God of the Kafirs and his Main 'Temple in the Parun Valley (Nuristan, South Hindu-Kush). Akten des 24sten Internationalen Orientalisten-Kongresses, München 1957. Wiesbaden 1959.

Edelberg, Lennart, 1960 a: Statues de bois rapportées du Kafiristan à Kabul ... Arts Asiatiques, vol. 7, p. 243-286. Paris.

Edelberg, Lennart, 1960 b: På sporet af skvatmollerne. Skalk 1960, 4. Aarhus.

Edelberg, Lennart, 1968: cf. Motamedi.

Edelberg, Lemart, 1968: Ard og àg i Nuristan (Ard and Yoke in Nuristan). KUML (Jutland Archaeological Society), Moesgaard, 8270 Højbjerg.

Edelberg, Lennart \& Lis Gramstrup, 1971: Synopsis of Contents \& Index to Sir George Scott Robertson: The Kafirs of the Hindu-Kush, London 1896 \& 1900. In collaboration with Kirsten Ewers Andersen \& Schuyler Jones. With a map by L. E., Jutland Archaeological Society, Moesgård,

9s Klaus Ferdinand, diary IV, 3: Požtit-mund [connected with the god] Jinimara [cf. Morgenstierne 1949: mltỉcu = wooden idol].

95 As far as I remember from 1964 Dogumrik was explained as the "wakil-1mara" and about Moni (Mlāndi) Robertson writes (1896 p. 399): In Presungul he [Moni] retains his rightful position in the Kafir Pantheon. Traditionally, he is the god always selected by Imrá [ = Mara] to carry out his orders to exterminate demons.

Qs IK. Ferdinand IV, 3: Pečak [connected with] Žuutwas [cf. Morgenstierne 1949 wëstli $=$ woman, wife]. [Could Zuutwas be $=$ Žiwudwëstli?].

Q7 A "hornstool" (sij)-kŭ) without carvings and a scarf (püjuruk) with cowrieshells, both from Lapact-tadba, are in the Ethnographical Collections of Moesgard. 
Elphinstone, Mountstuart, 1815: An Account of the Kingdom of Caubul . . ., London.

Jones, Schuyler, 1966: An Annotated Bibliography of Nuristan (Kaflristan) and the Kalash Kafirs of Chitral. Part I. With a Map by Lennart Edelberg. The Royal Danish Academy of Sciences and Letters: Hist. Filos. Medd. Dan. Vid. Selsk. 41, no. 3 (1966). Copenhagen. Jones, Schuyler, 1967: The Political Organization of the Kam Kaflrs. A Preliminary Analysis. Ibidem 42, no. 2.

Lentz, Wolfgang, 1939: Zeitrechmung in Nuristan und am Pamir. Abhandlungen der Preussischen Akademie der Wissenschaften, Jahrgang 1938. Berlin.

Morgenstierne, Georg, 1949: The Language of the Prasun Kafirs. Norsk Tidsskrift for Sprogvidenskap, vol. 15. Oslo.

Morgenstieme, Georg, 1951: Some Kati Myths and Hymns. Acta Orientalia, vol. 21, p. 161-189. Copenhagen.

Morgenstierne, Georg, 1954: The Waigali Language. Norsk Tidsskrift for Sprogvidenskap, vol. 17. Oslo.

Morgenstierne, Georg, 1967: Some Folk-Songs from Nuristan. To honor Roman Jakobson, Essays on the Occasion of his 70th Birthday, 11. Oct. 1966. Vol. 2. The Hague.

Morgenstieme, Georg, 1968: Mythological Texts from the Kates of Nuristan. Mélanges d'Indianisme à la mémoire de Louis Renou. Publication de l'institut de civilisation indienne. Paris.

Motamedi, Ahmad All and Lemart Edelberg, 1968: A Kaflr Goddess. Arts Asiatiques, vol. 18, p. 3-21. Paris.

Robertson, Sir George Scott, 1896: The Kaflrs of the Hindu-Kush, London. (2nd ed. 1900).

Snoy, Peter, 1962: Die Kaftren: Formen der Wirtschaft und Geistigen Kultur. Giessen.

Manuscripts:

Alvad, Thomas: Liste I, Fortegnelse over Nuristan optagelser 1947-49 og 1953-54 (in The Danish Folklore Archives, Copenhagen S.).

Barrington, N. J., 1960: Account of a 'Tour to Two Valleys in Nuristan by the Oriental Secretary in July, 1960. 25 pages.

Edelberg, Lennart: Tape-recordings from Nuristan 1964 (in the Danish Folklore Archives, Copenhagen S.).

Edelberg, Lemnart: Diaries $1948,-49,-53,-54$, and -64 .

Ferdinand, Klaus: Diary I-V Nuristan 1953.

Heath, J. M., 1963: Account of a Tour to Bashgul, Kantiwar, and Parun.

Motamedi, Ahmad Ali: Diary Nuristan 1953. 


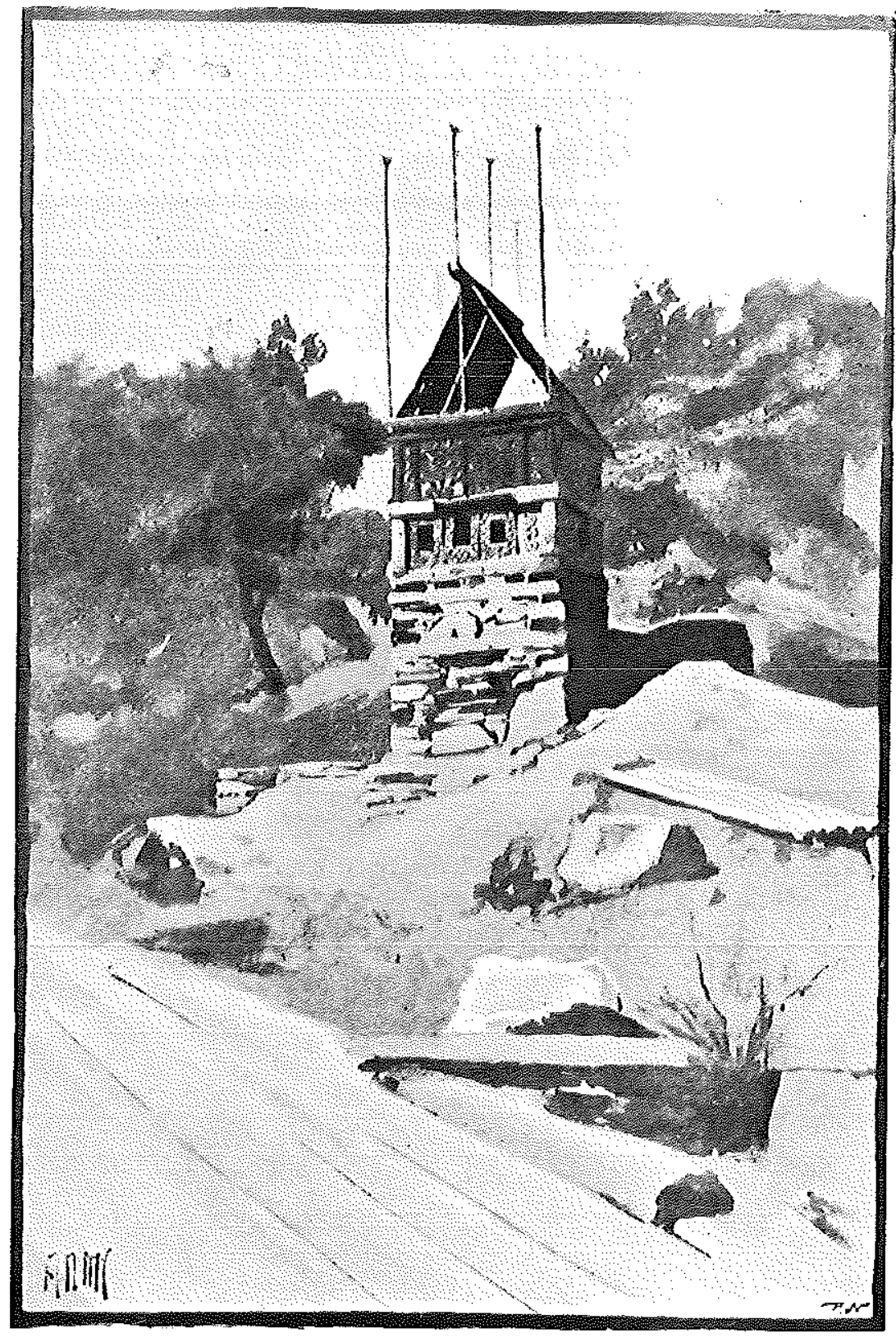

Figure 1. Dizane's temple at Kamdesh. (After Robertson 1896 p. 396). 


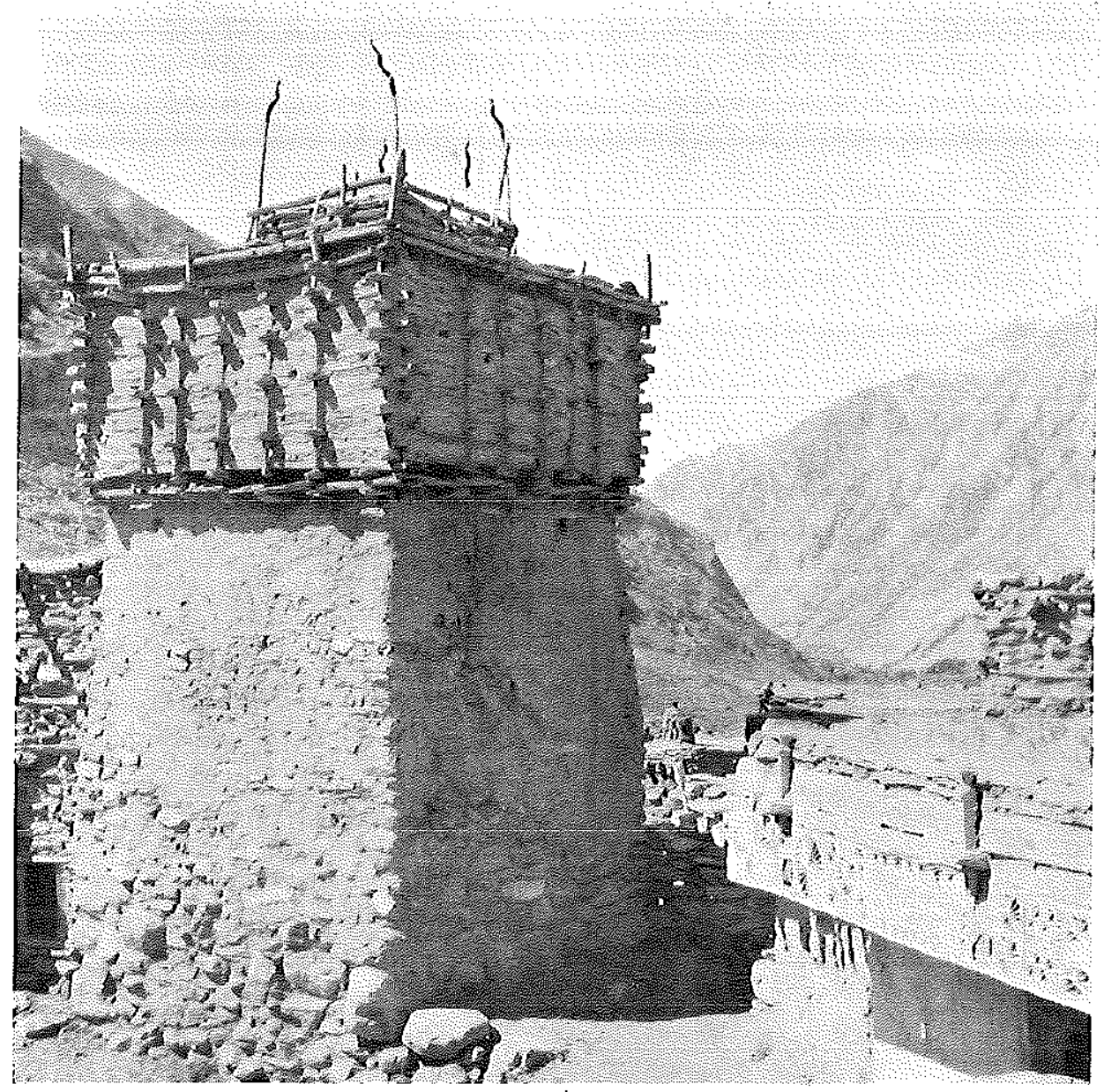

Figure 2. The village tower of Shtiwe wilh markhor horns on the four poles on the top. To the right is the mosque. L. E. phot. June 17, 1948.

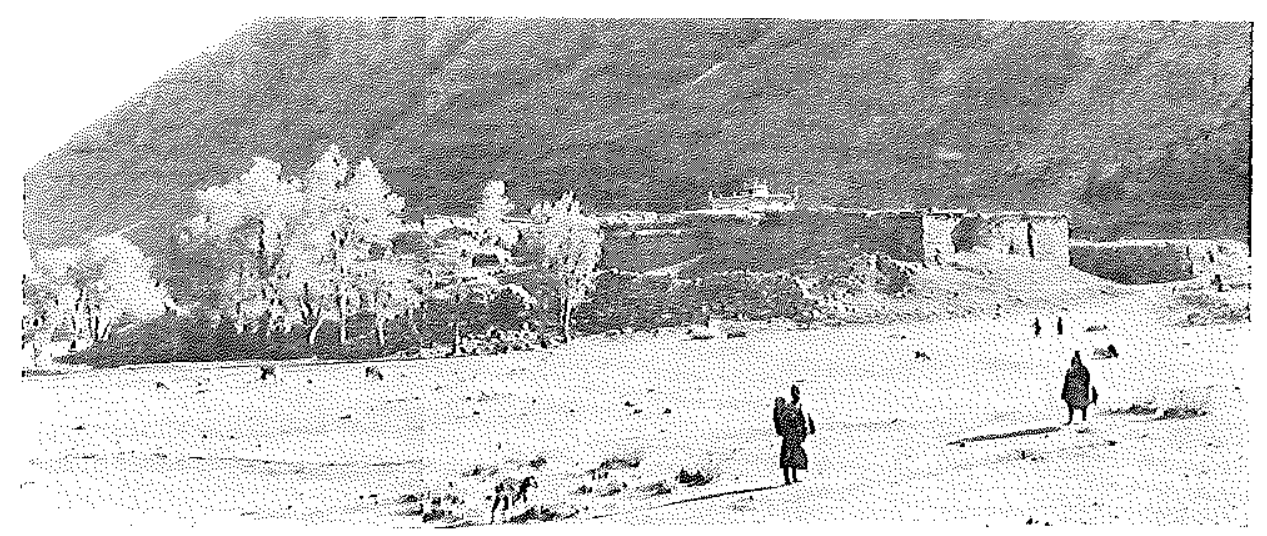

Figure 3. The tèpe of Pronz. 1., F. phot, Oct. 1953. 


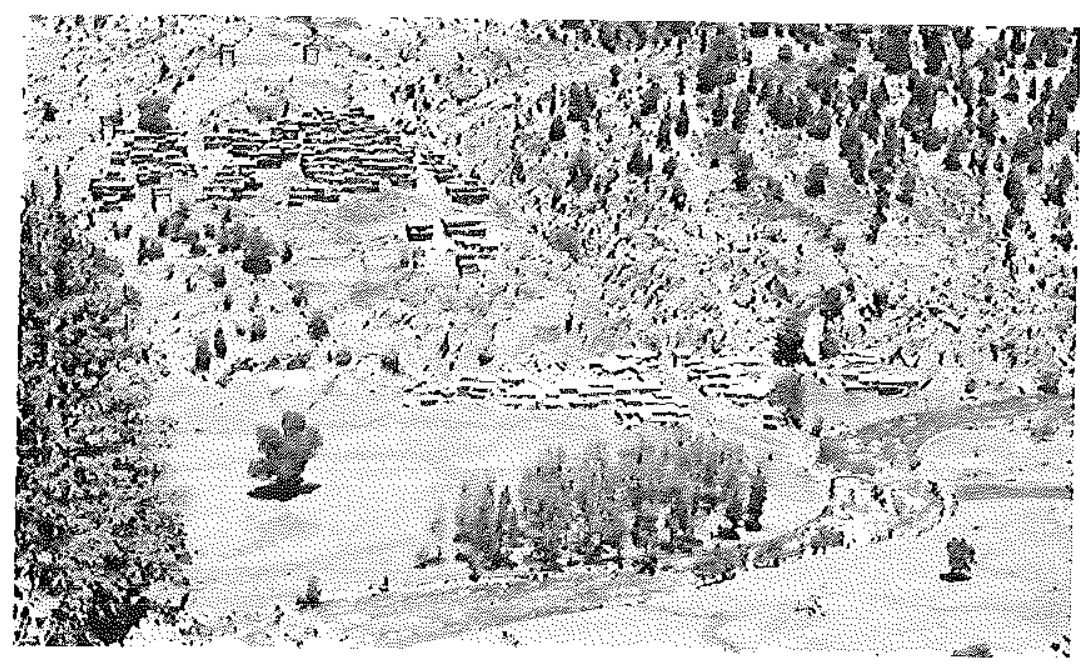

Figure 4. Pashki. Above the village the two towers of Ram-ytu are visible. Tho the left two other towers are seen at different levels. In the lower right hand conce of the village a few hlacksmith families hatve their houses. At the foot of the eliff the winter pens are situated. L. E. phot. Jume 19.18.

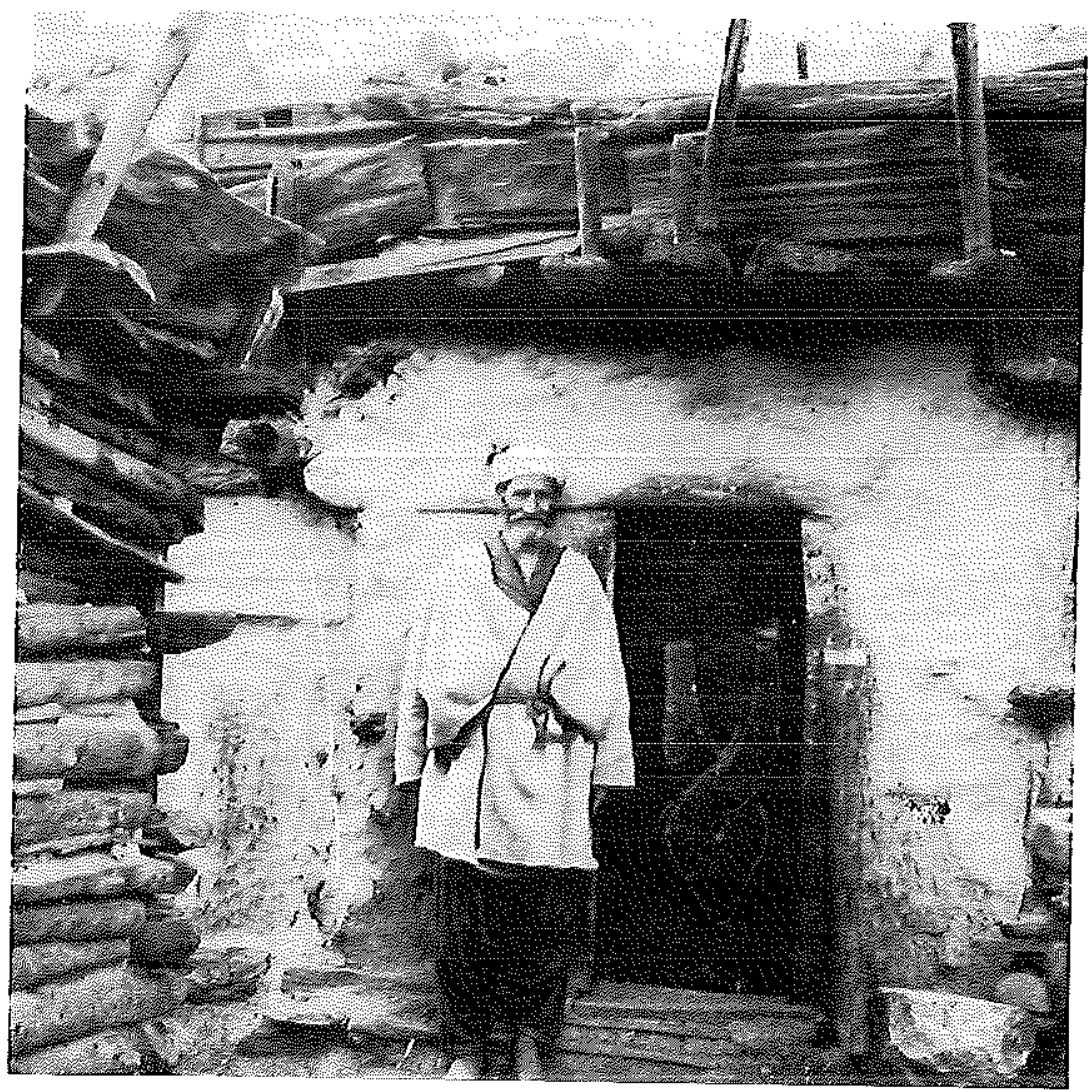

Figure 5. The entrance to the lemple of Disni. To the left is mulla Mirmad (Kafir name: Pstimul), the owner of the house. Pashki. L. E. phot. July 30, 1964. 


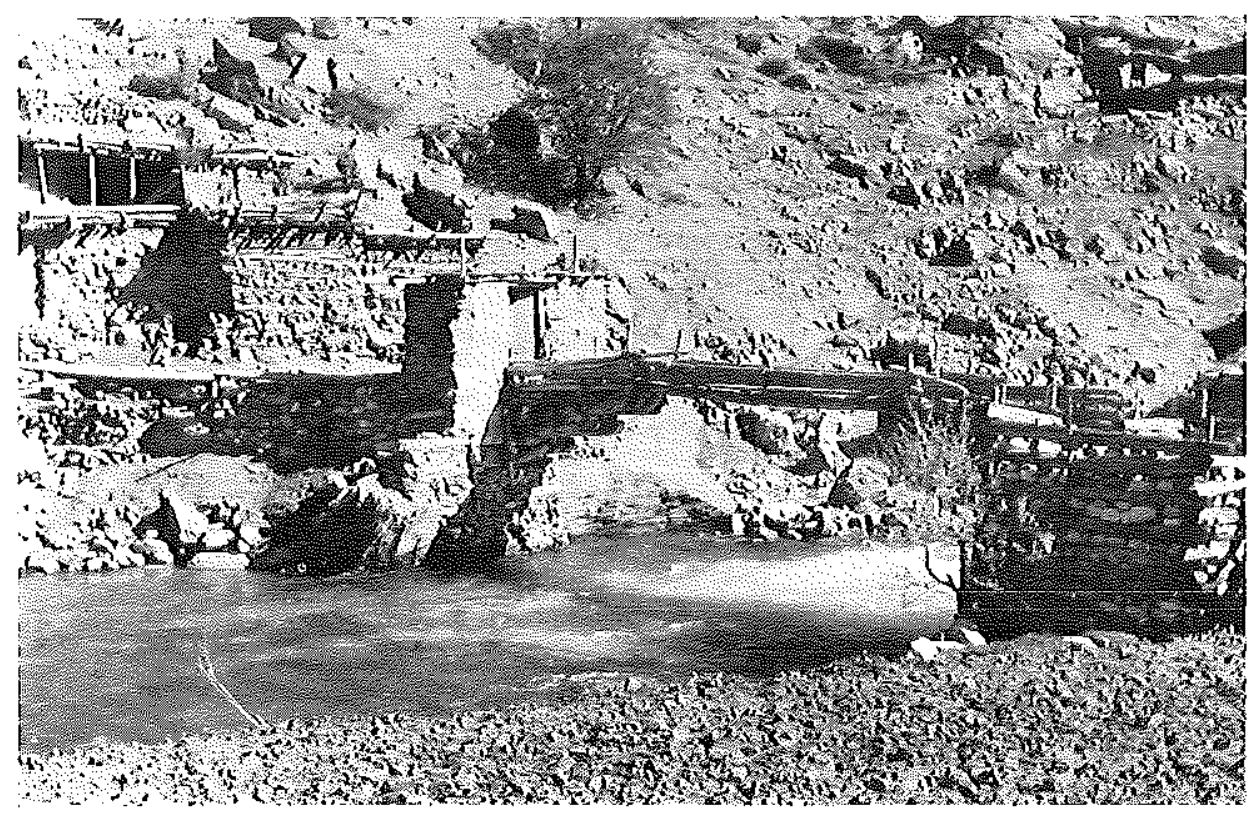

Figure 6. The bridge of Zumu. L. E. phol. Maty 27, 1948.

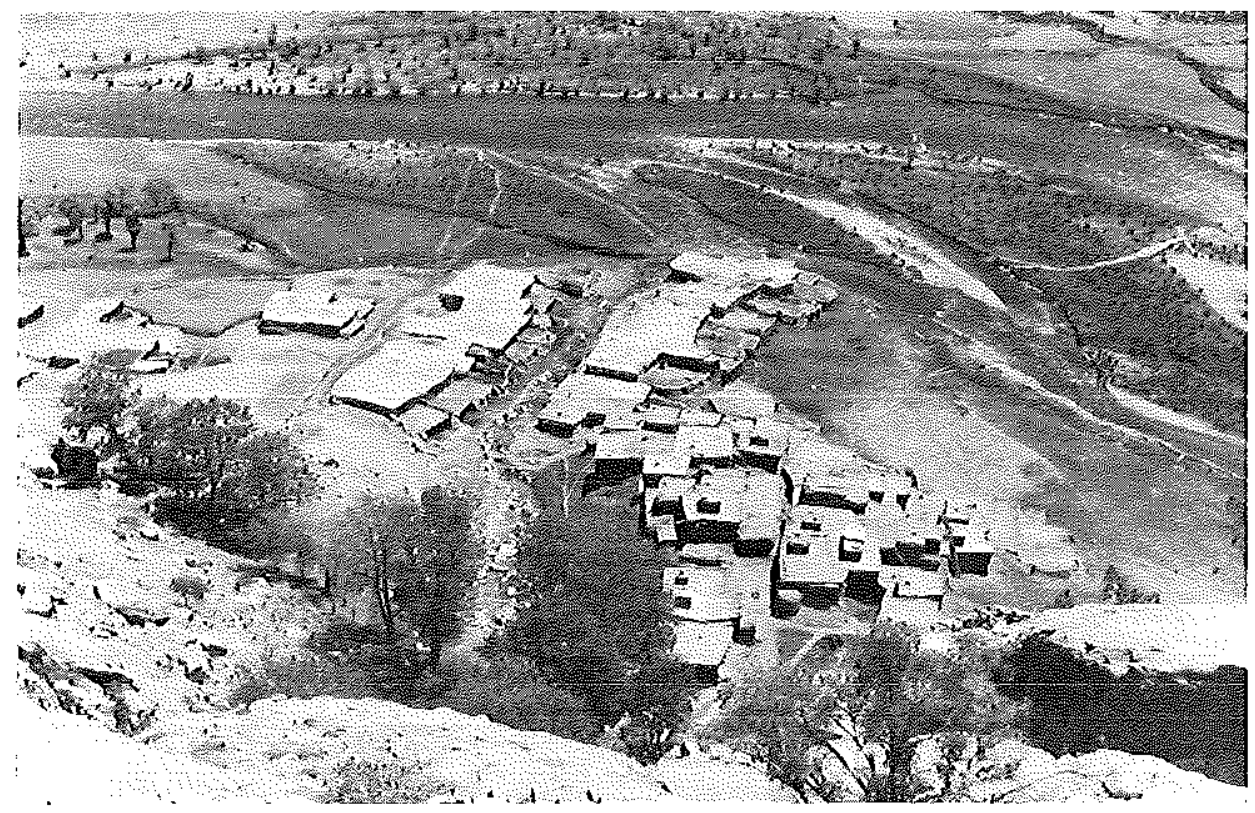

Figure 7. Kushteki seen from the stronghold above the village. In the centre the dry bed of "Imra's water". 'To the right, the houses; to the left, the winter pens. To the left is also seen the site of the chief temple of Imra (half covered by trees) and in front of this the flat area of the eastern colonnade mentioned by Robertson 1896 p. 390. From the north-eastern corner of this area a path leads to the Islamic place of prayer on the river bank (cf. fig. 11). Between the trees on the meadow to the left there was an entrance to the nether world (Robertson 1896 p. 393). L. E. phot. May 27, 1948. 


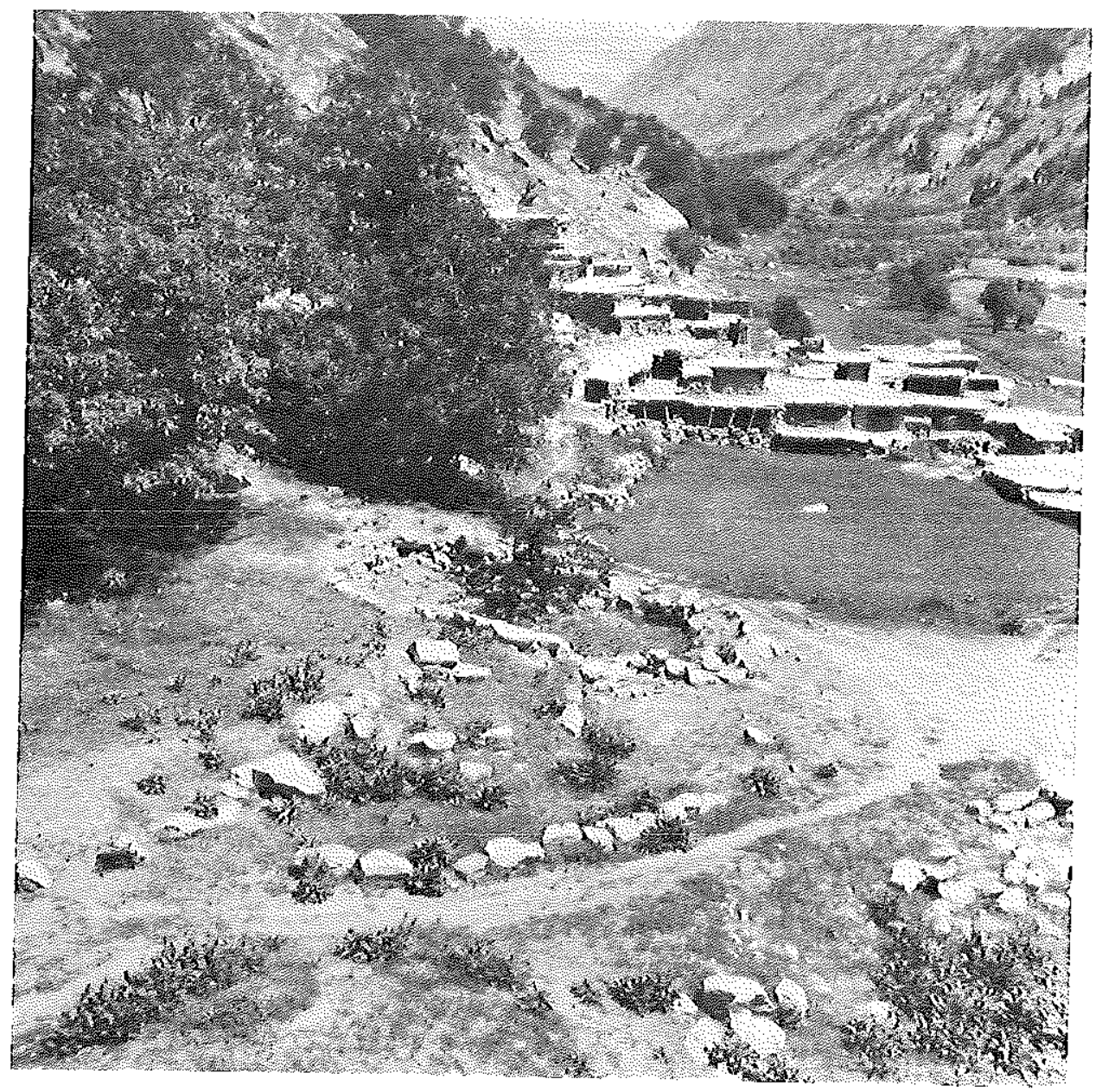

Figure 8. The site of the chief temple to mma seen from the south. To the right (on the temple's east side) is the area which was occupied by the square portico "forming a kind of rough colonnade" (Rob. 1896 p. 389--392). IKushteki. L. E. phot. .Iuly 19, 1940. 


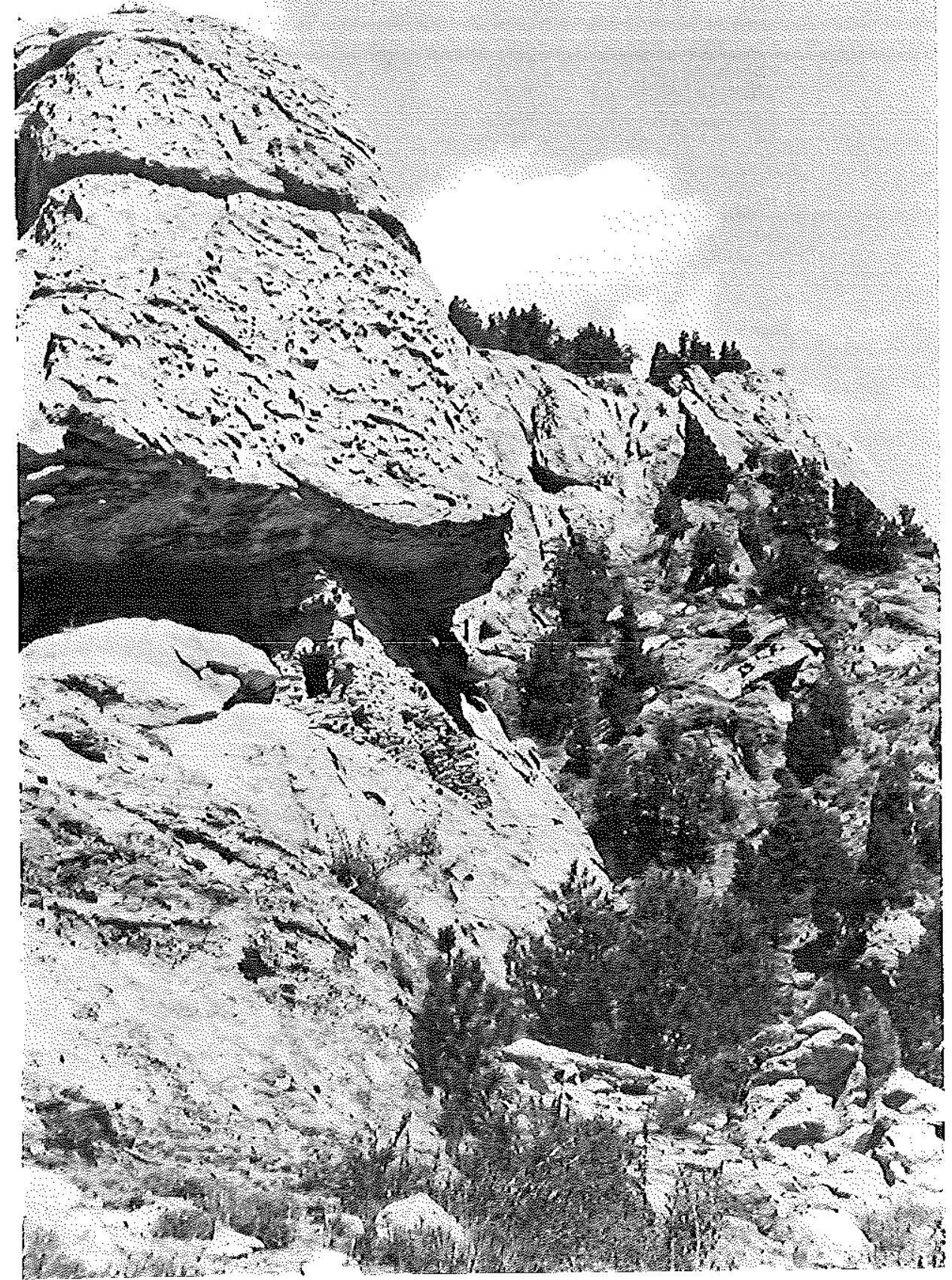

Figure 0 . The stronghold above Kushteki. 'The entrance leads to a big cave under the rock. I.. E. phot. May' 27, 1949. 


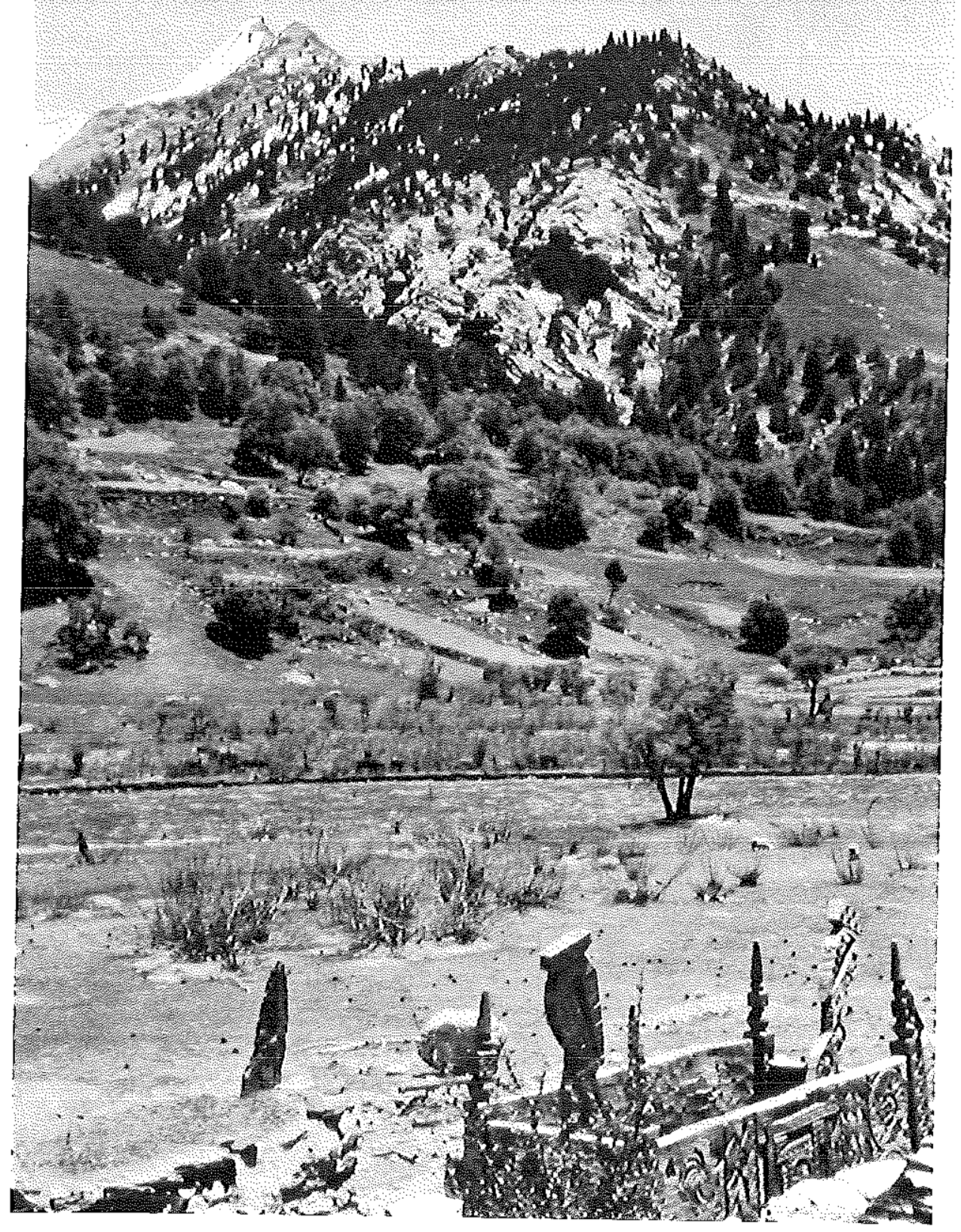

Figure 10. The cemetery of Kushtekt. In the foreground the carved lomb of Palyuk, to the left of that the rocky tomb of his son Jan Gul. In the background the beattiful snow-covered peak of Süskümünt. L. F. phot. Oet. 1953. 


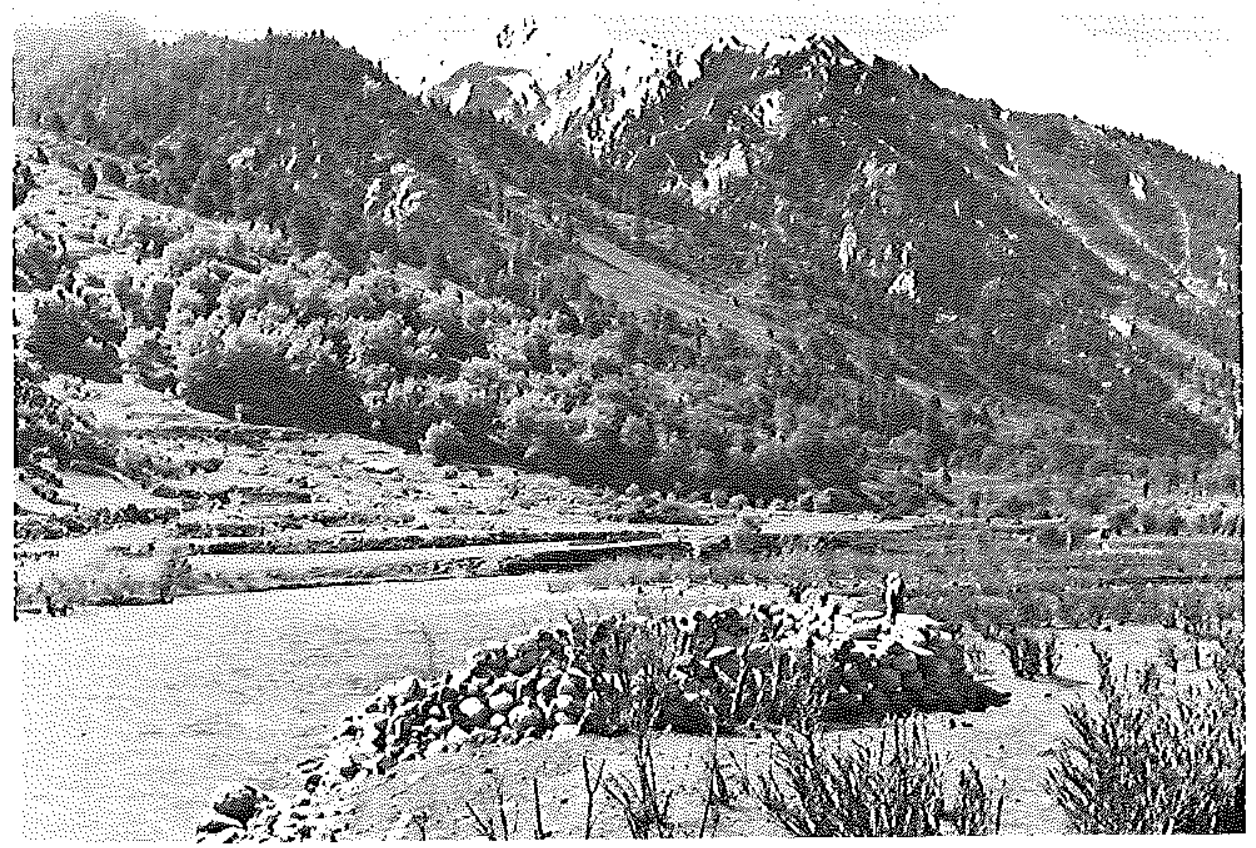

Figure 11. Islamie place of prayer at the river side (cf. flg. 7). The kibla is indicated by an upright stone. Kushteki, L. E. phot. May 27, 1948.

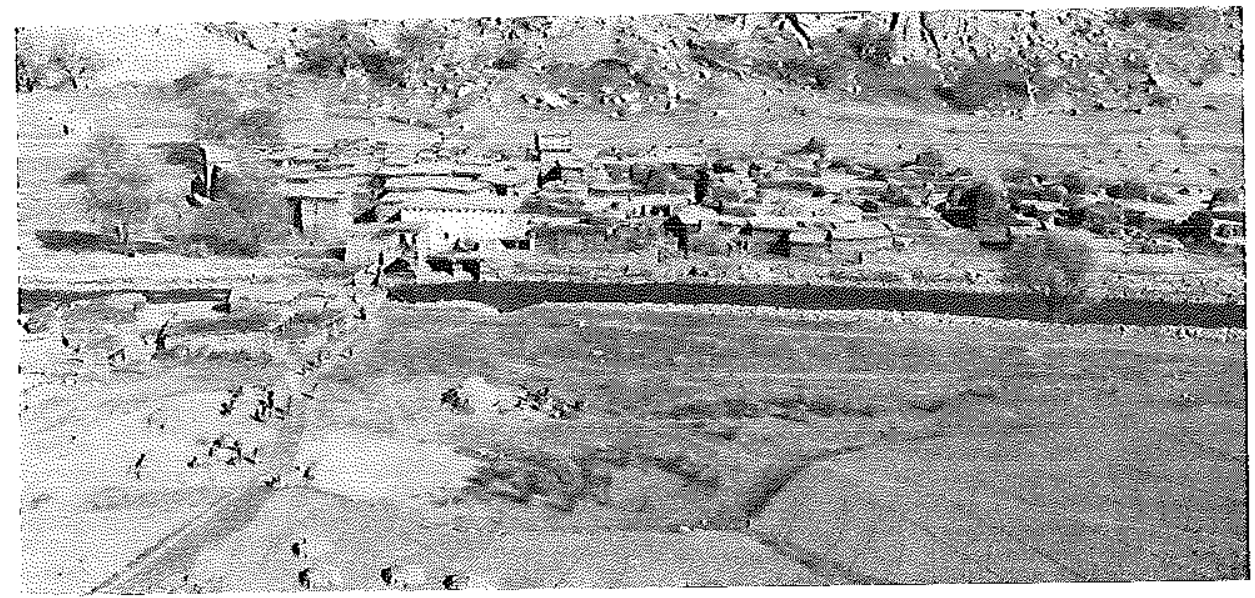

Figure 12. Dewa seen from the west. The village has a northern and a southern tower. South of the southernmost lower are the winter pens. Other winter pens are built on the right side of the river (left foreground). In front of these the men and women are busy finishing the threshing before the end of the month of kila. L. E. plot, Oct, 22, 1953. 


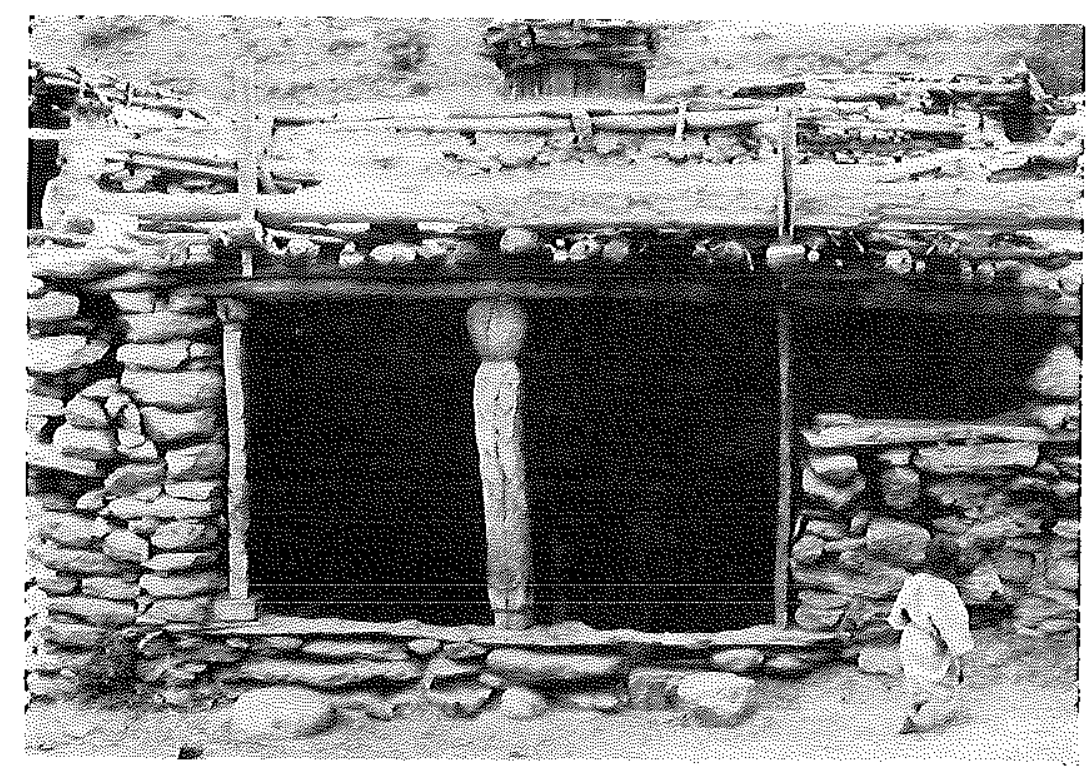

Figure 13. The open east side of the amal (assembly hall) in Dewa. L. E. phot. July 29, 1964.

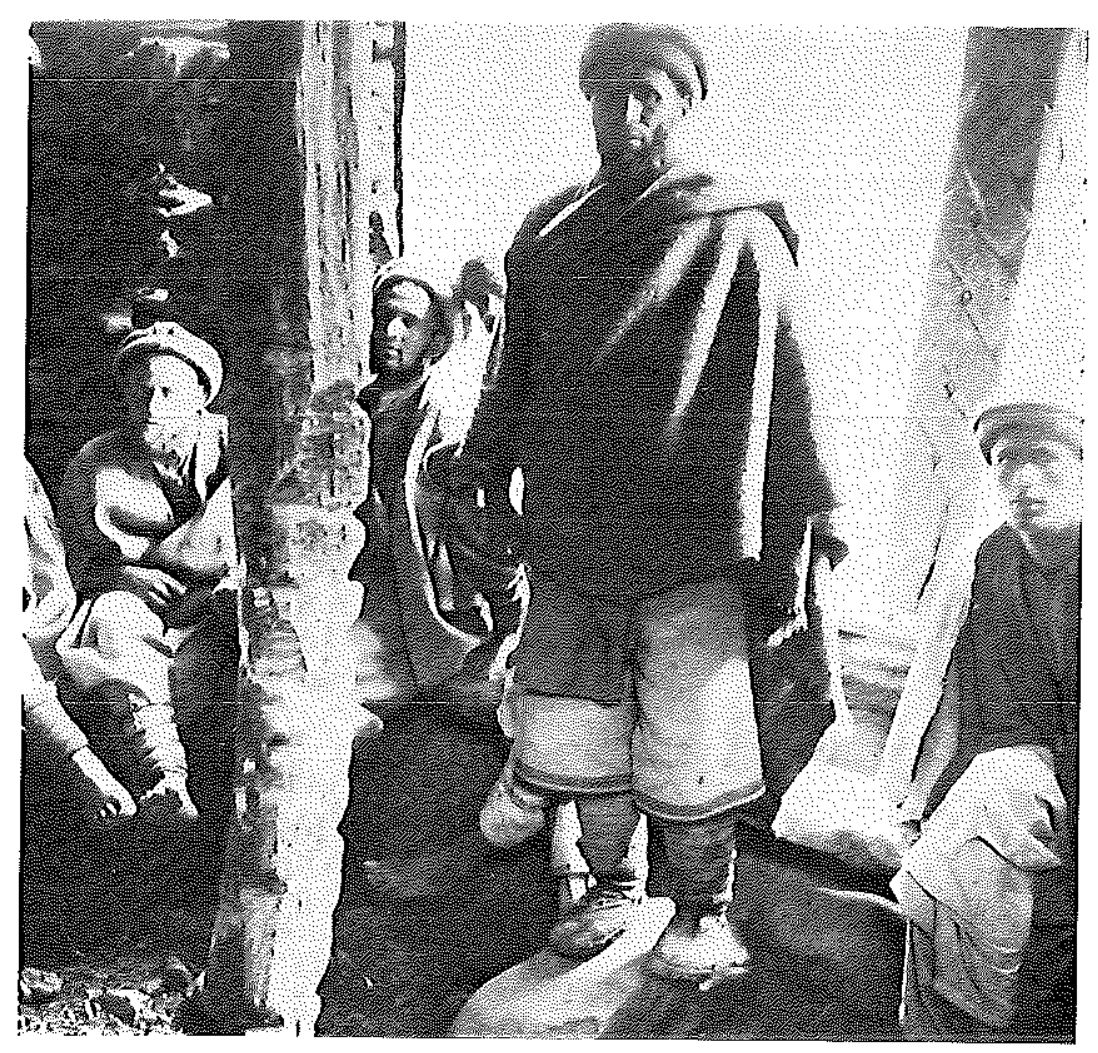

Figure 14. A man stands in the speaker's place in the anul on the flat stone between the central pillar and the pillar on the open side. L. E. phot. July 29, 1964. 


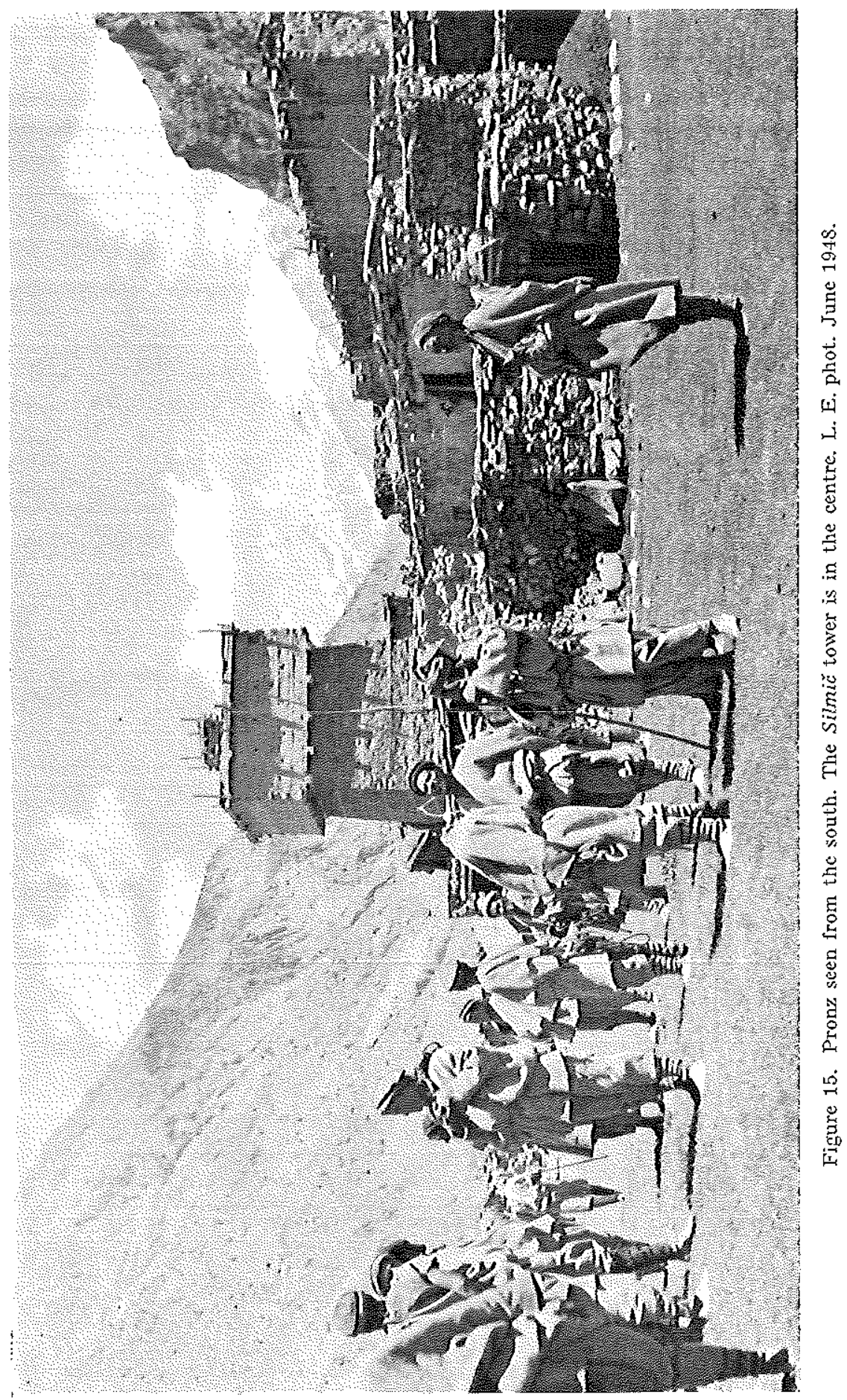




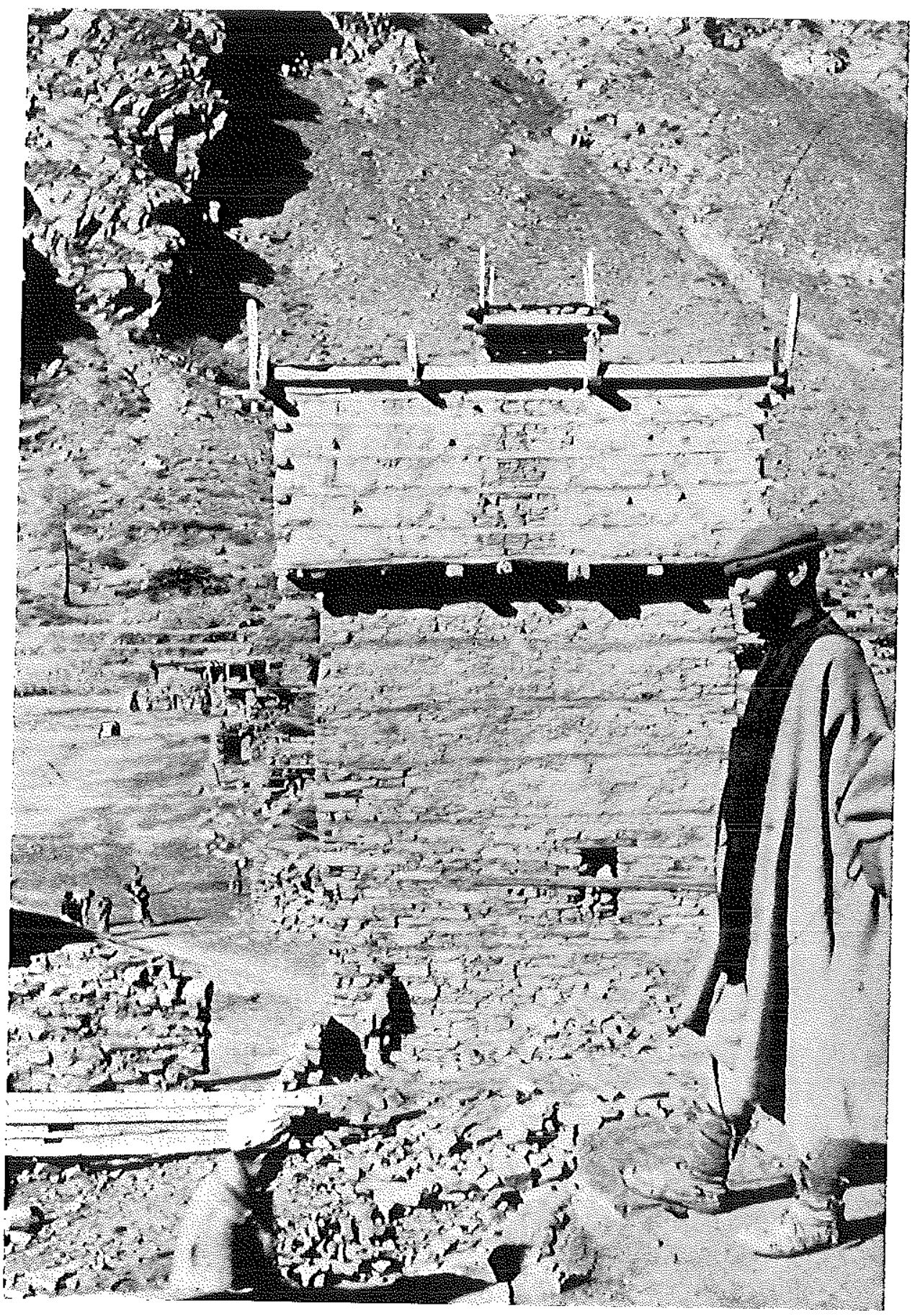

Figure 16. The Silmid tower seen from the east. In the background to the left is the stone of Cinduza in front of the winter pens. Pronz. L. E. phot. Oct. 1953. 


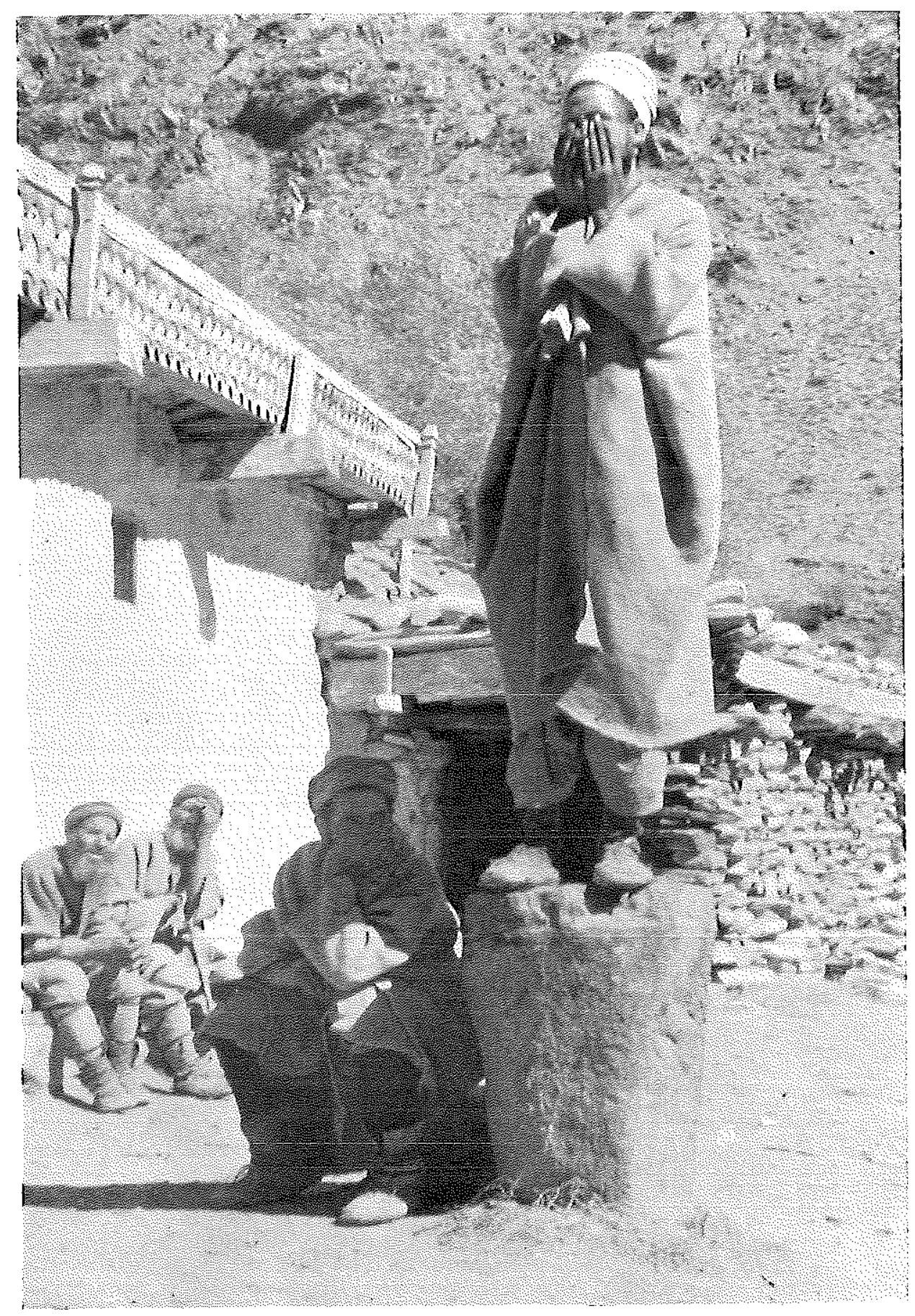

Figure 17. The stone of Ashpegra (G. B.: Espereg-rä) in front of the mosque. The mulla is calling the villagers to prayer, Pronz. Klaus Ferdinand phot. Oct. 1953. 


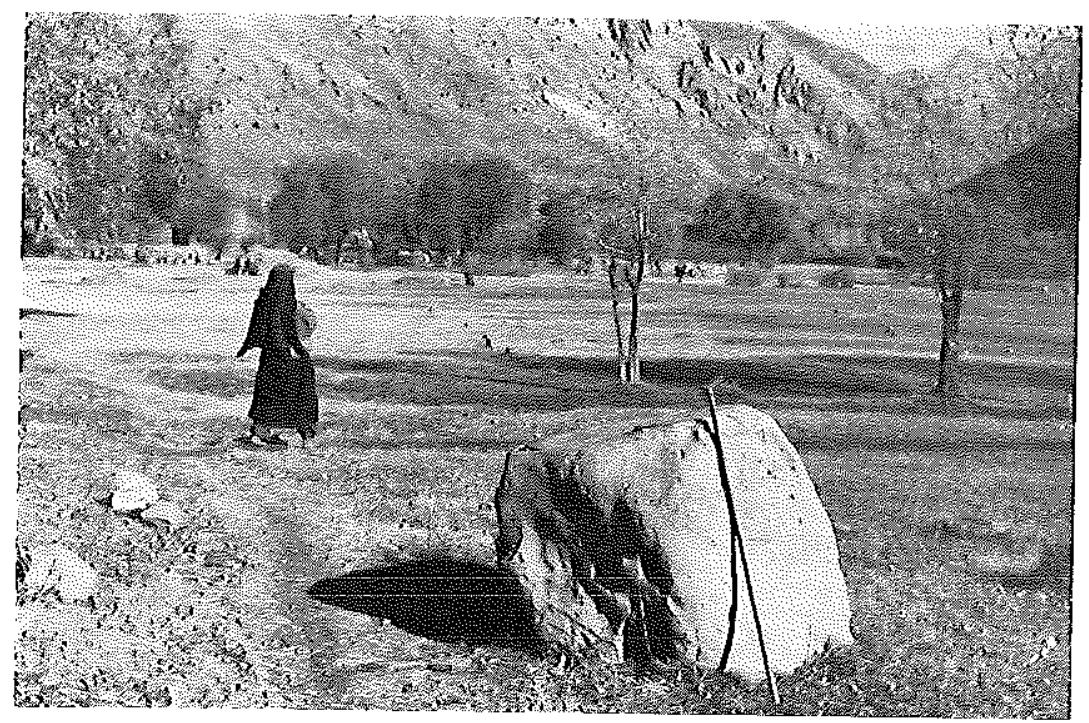

Figure 18. The stone of Gumra-kot east of the village of Pronz, L. E. phot. Oct, 1954.

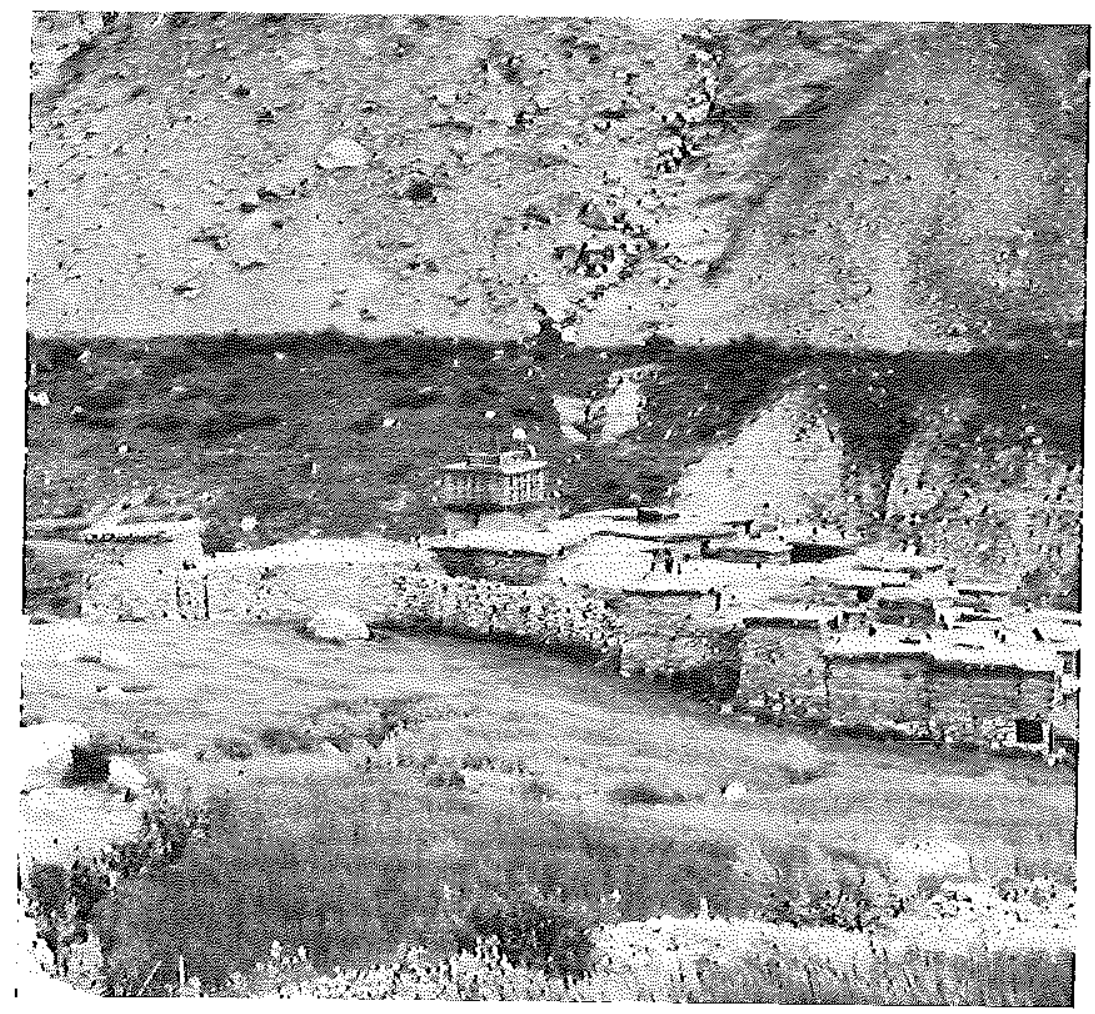

Figure 19. Shtiwe seen from the west. To the right of the tower the big louver on the flat roof of the temple of Mära. On the mountain slope behind rums the irrigation chanuel satd to have been made by Disni. As it lcaks, the ground turns green beneath the cliannel. L. E. phot. JuIy 1964. 


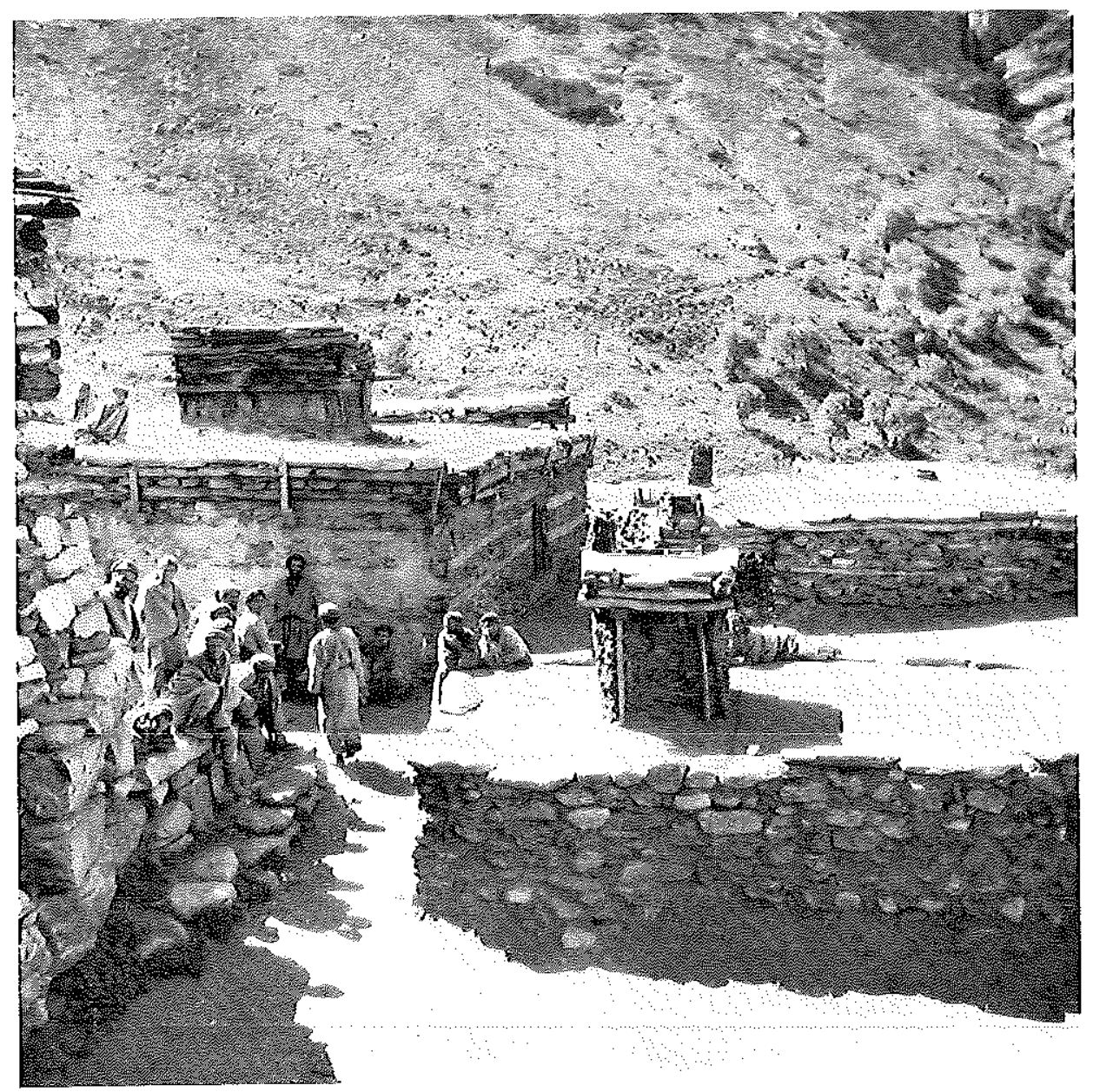

Figure 20. The Mărt-temple seen from the west. Shtiwe. L. E. phot. June 17, 1948 . 


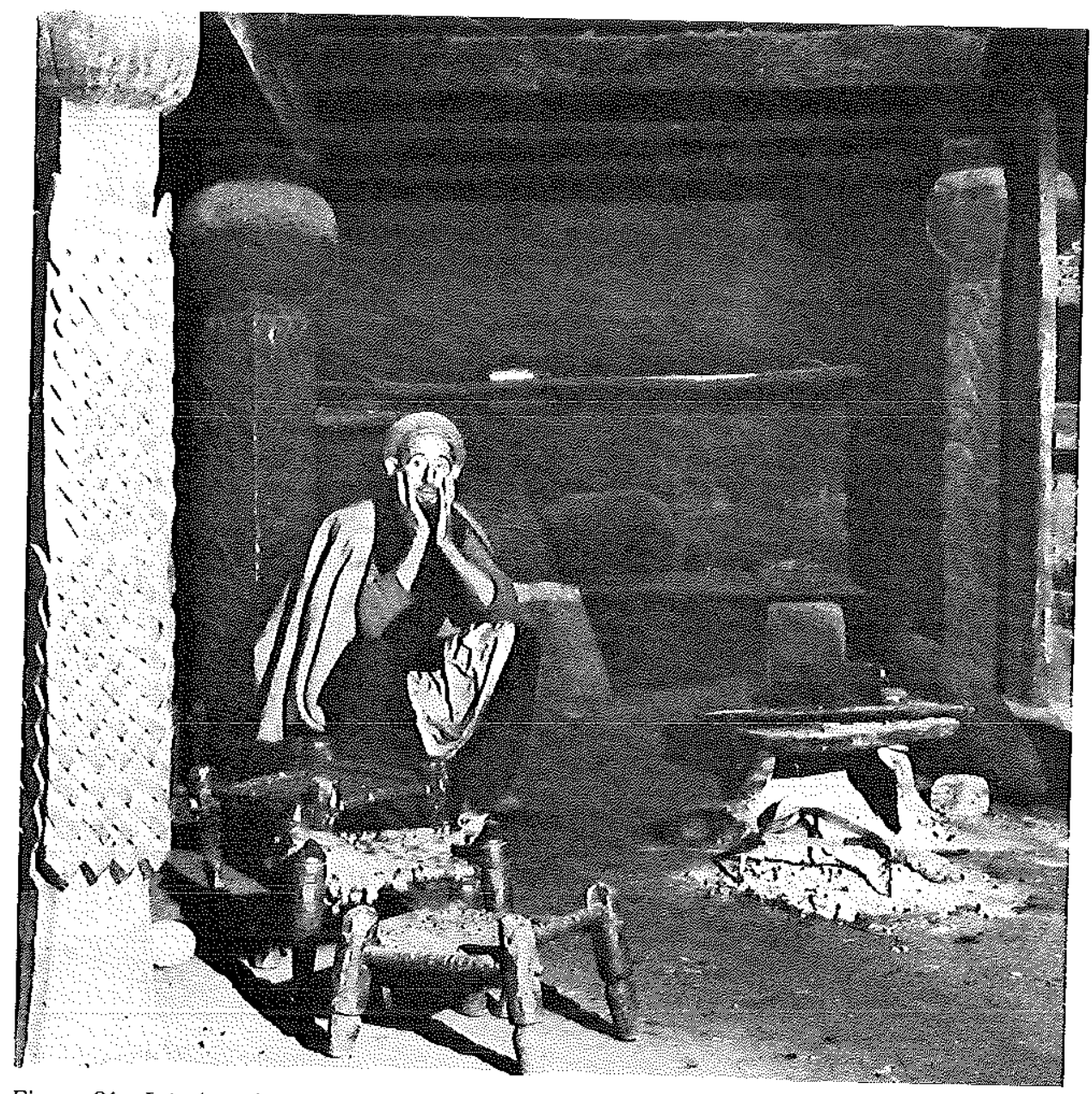

Figure 21. Interior of the Matratemple seen from the east. Kusum, the formal heat of the clan and brother of Zäman, is silting at the frephace, which is situated between four carved pillars, three of which are visible. Behind the flrephace a short stone pillar where presumably the idol was placed in Katfir time (cf. Motamedi, A. A. and fennat Edeherg 1968). Shtiwe. I. E. phot. Aug. 1, 1964 . 


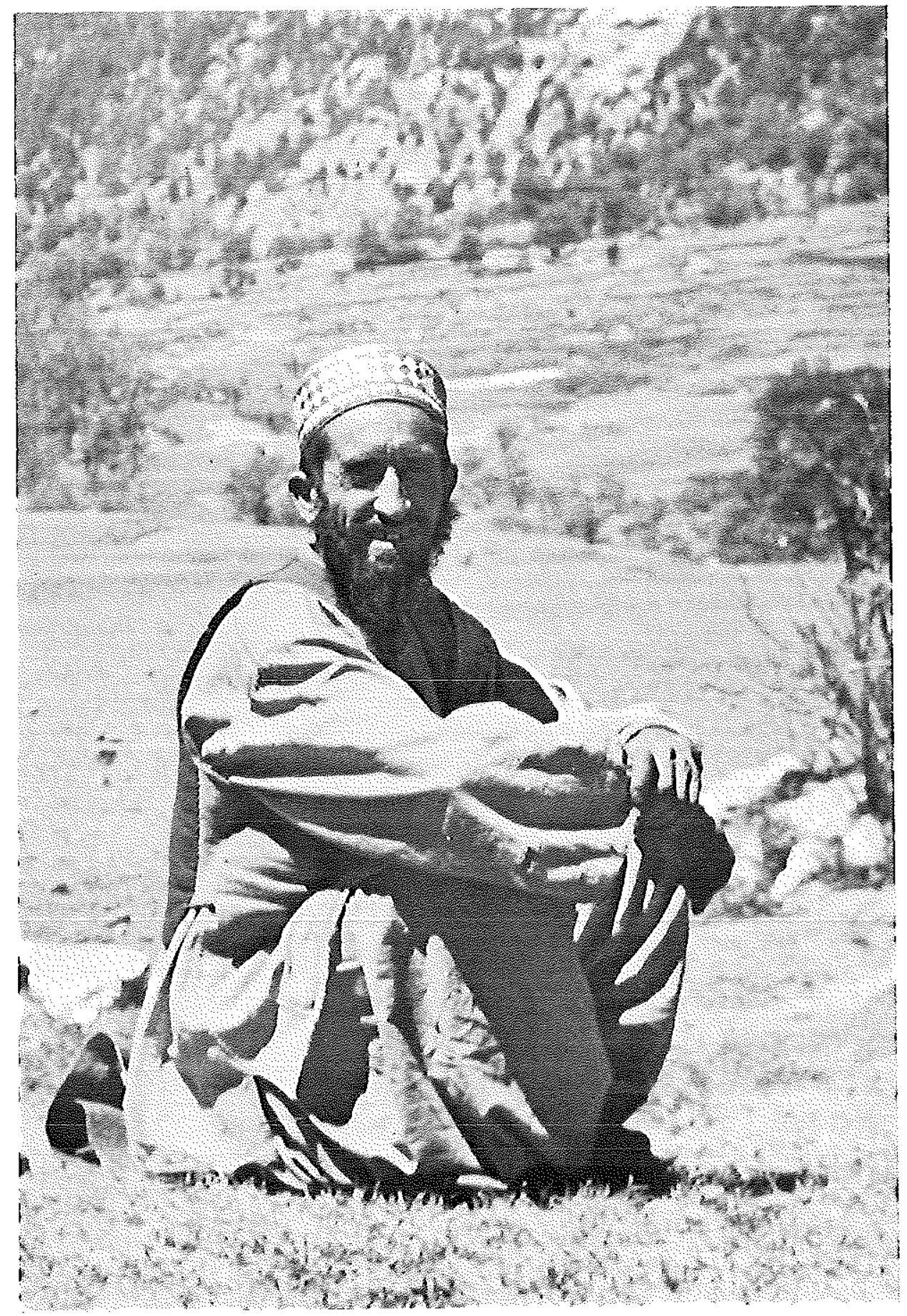

Figure 22. Amir Shah, the blacksmith. Pashki. Akbar phot. June, 1948. 


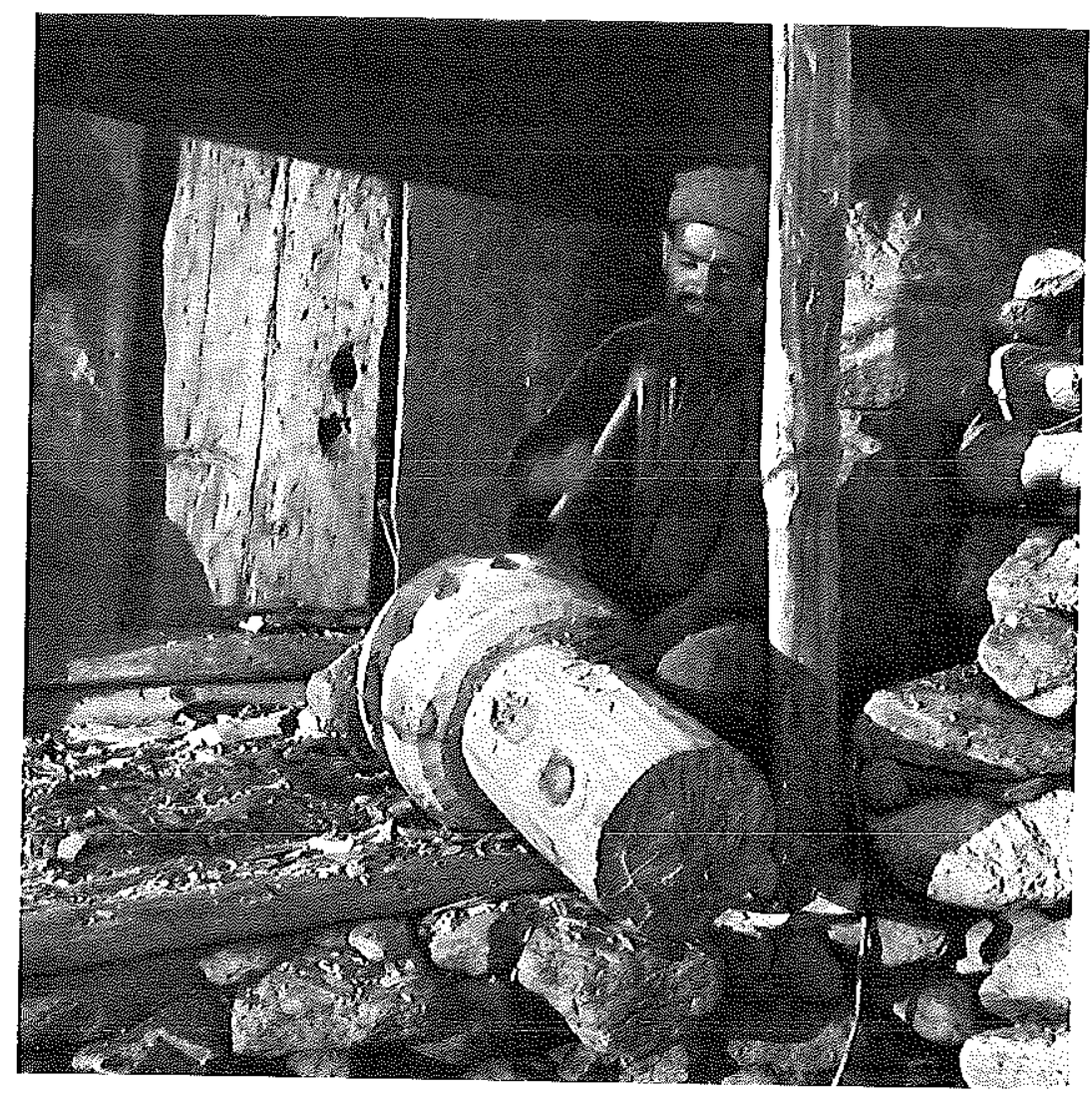

Figare 23. The son of Gul Mohammad, the blacksmith, trimming the erown wheel of a borizontal water mill (cf. Lennart Edelberg 1960 b). Pashki. Klaus Ferdinand phot. Oct. 1953. 


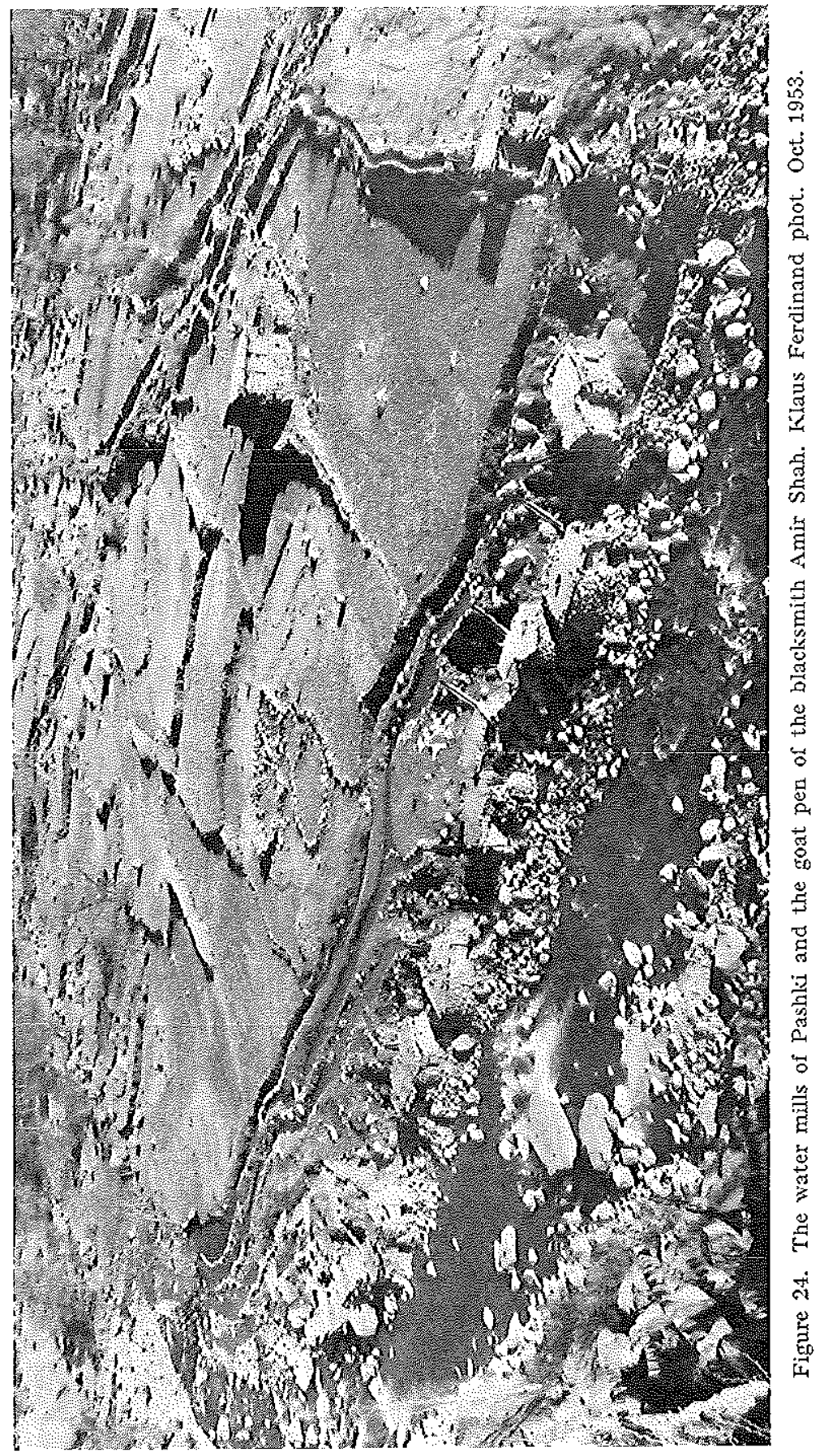




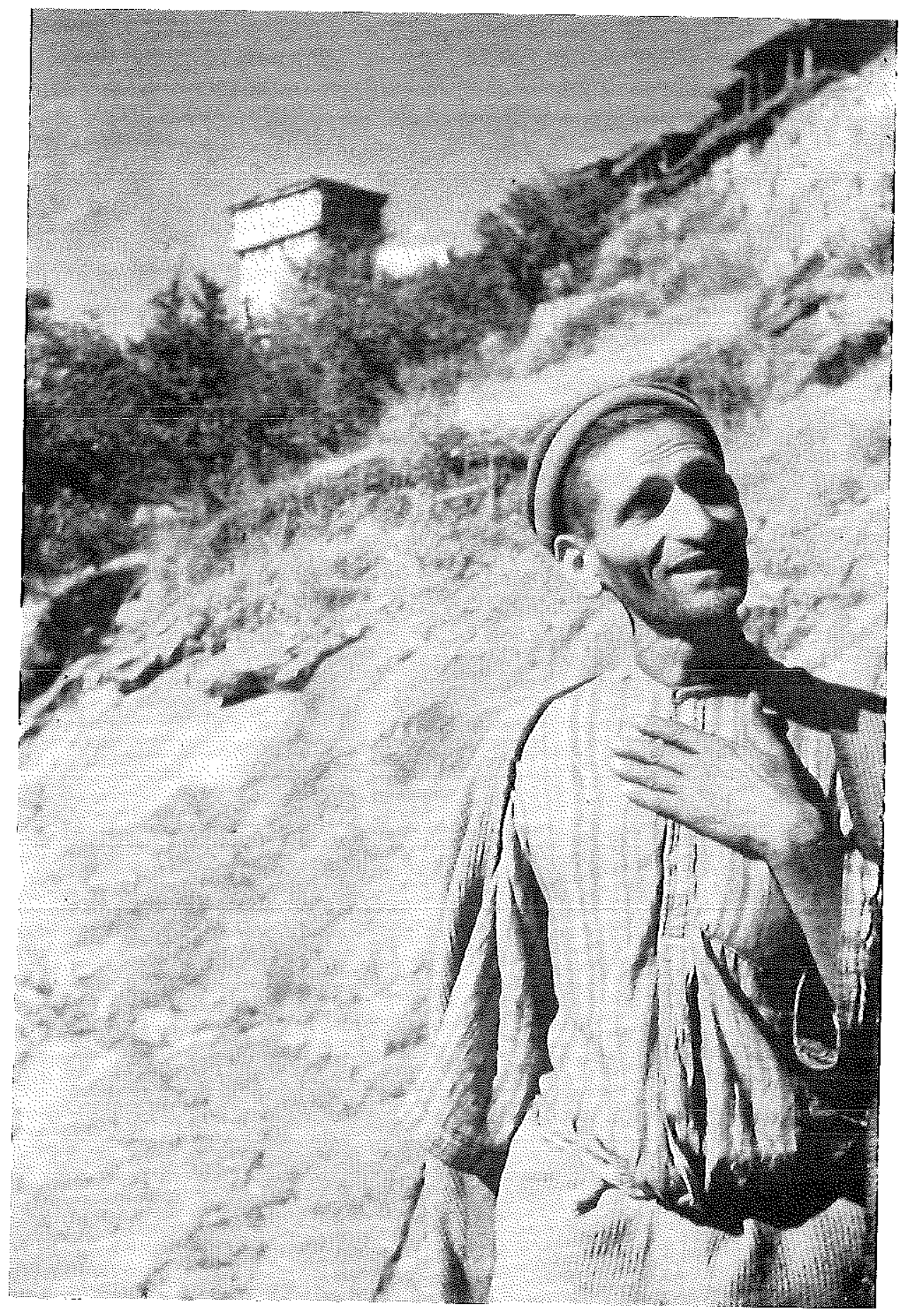

Figure 25. Abdur Rahim. Pashki. L, E. phot. Oct. 1953. 


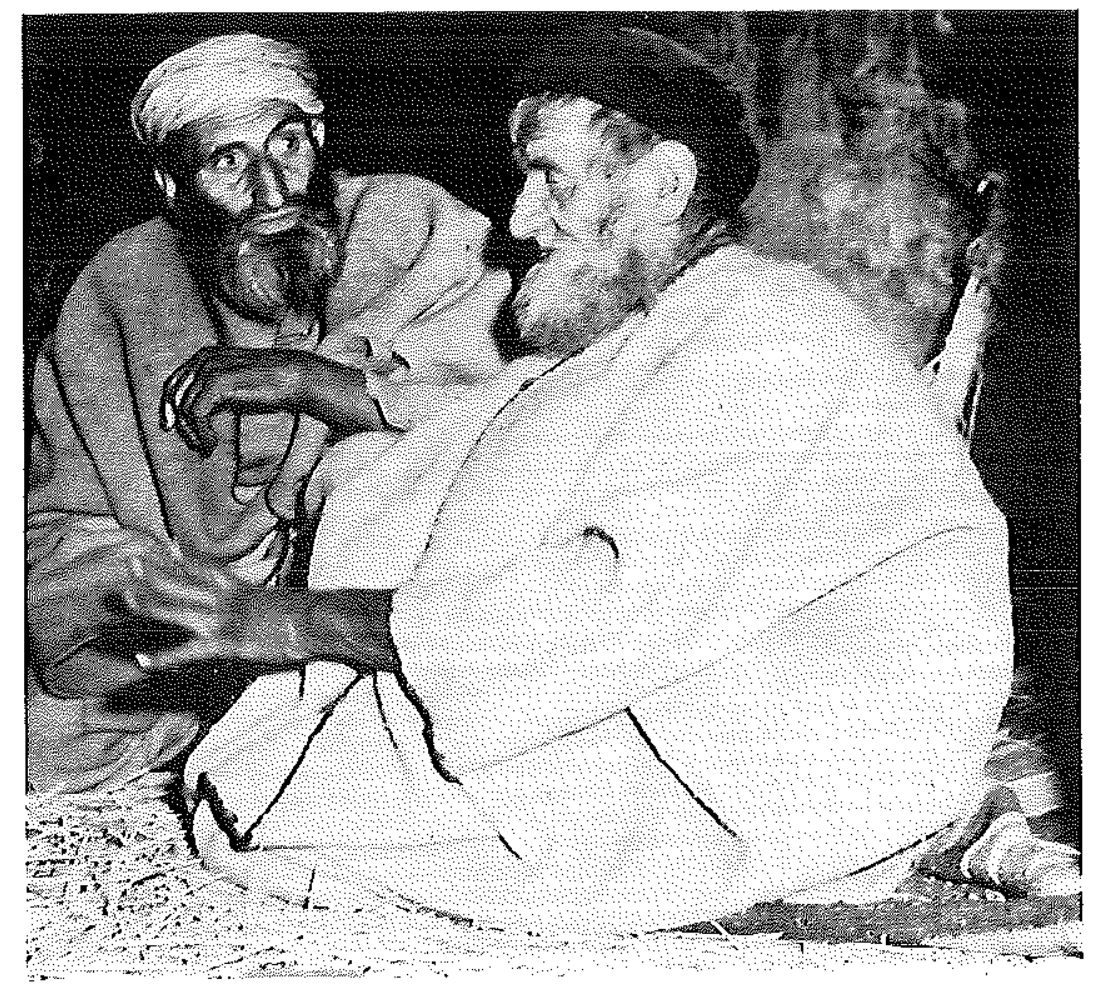

Figure 26. The blind Wodi singing the hymm no. 5. To the left the malik of Kushteki. The picture was taken in the guest house, but Wodi acts as if he were in the chief temple of Imra. Peter Rasmussen phot. Oct. 1953.

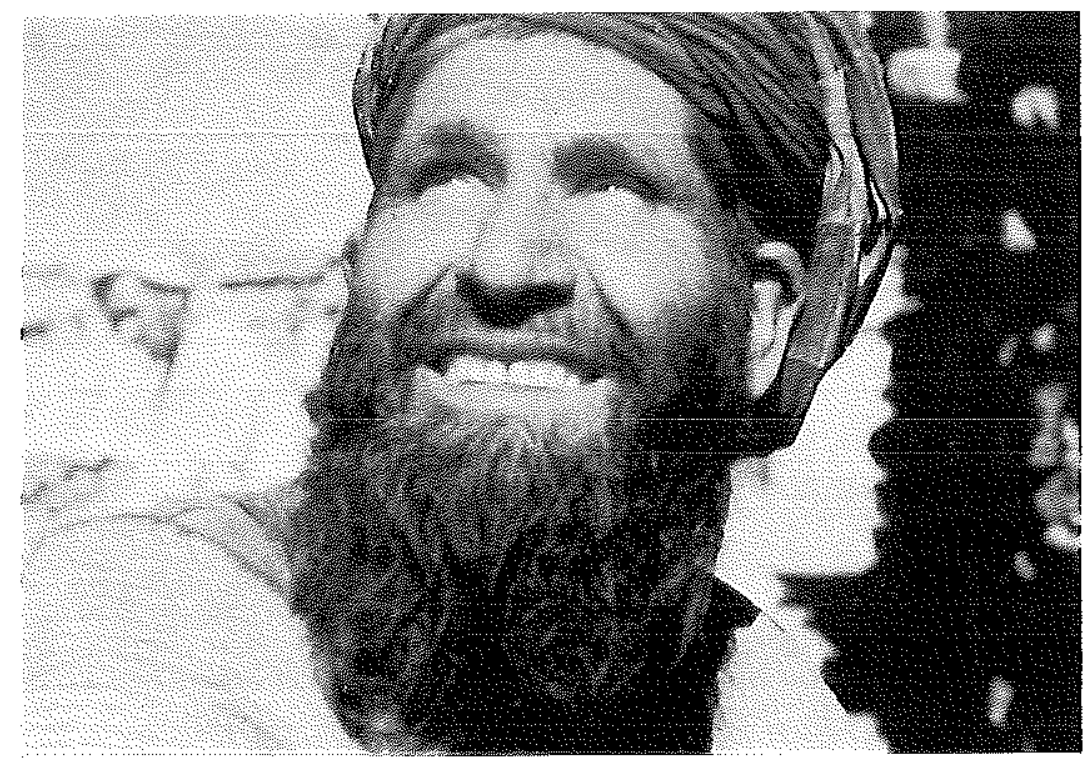

Figure 27. Abdulla from Pronz. Klaus Ferdinand phot, Od. 1953. 


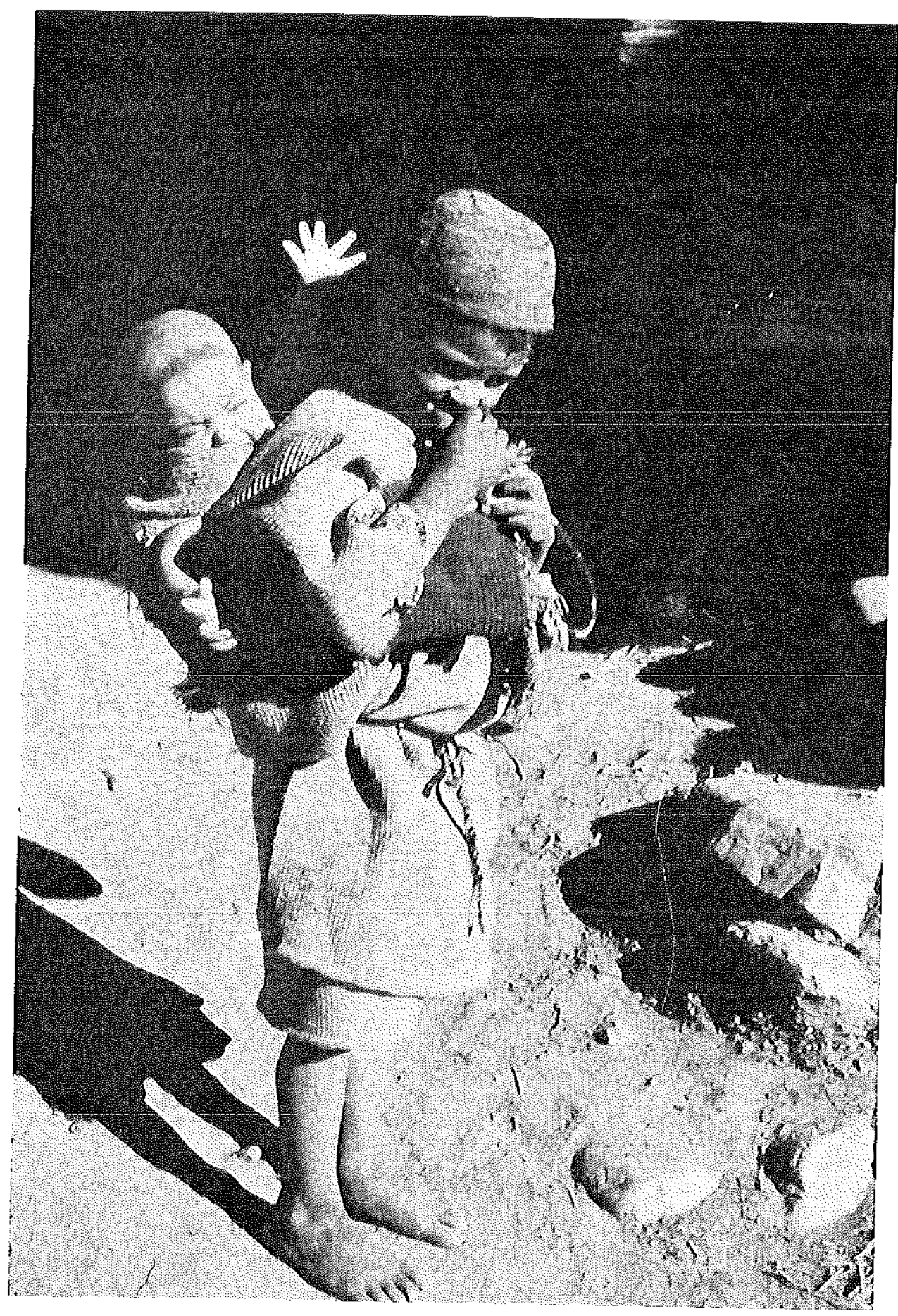

Figure 28. Boy carying a baby. L. E. phot. Oet. 1953. 


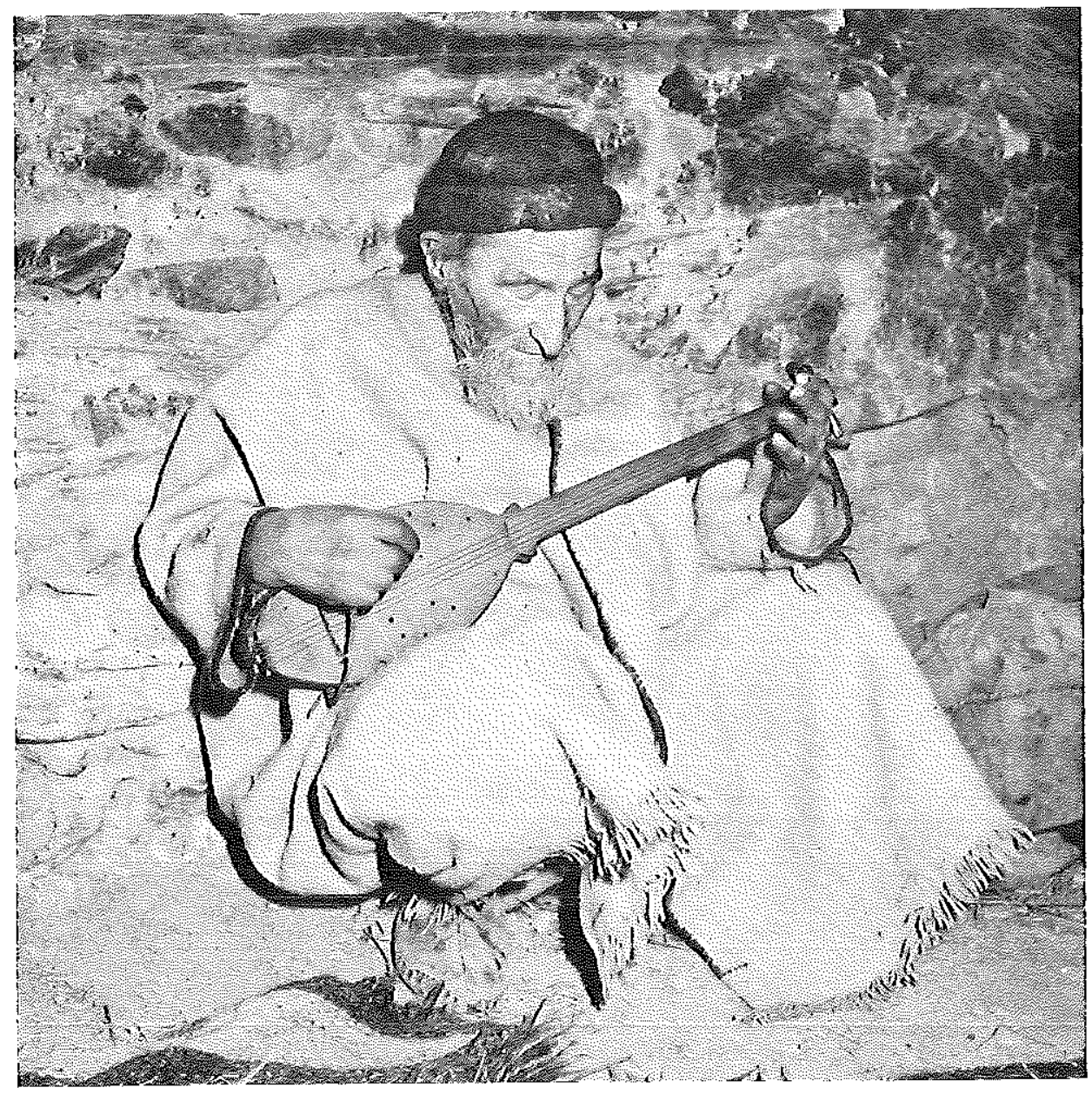

Figure 29. Wä̉ playing his four-stringed guitar (Paruni: $u r^{\prime} b \bar{a}$ ) and humming the hymms published here. Shtiwe. Peter Rasmussen phot. Oet. 1953. 


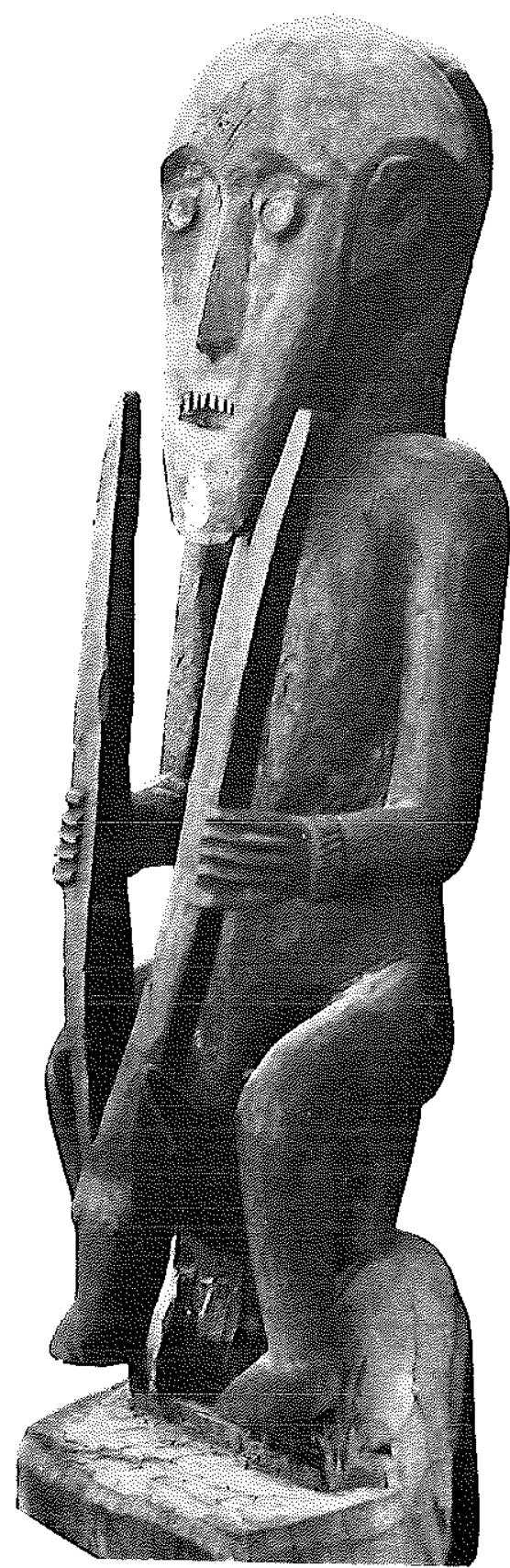

Figure 30. The goddess Disni from Shtive silling on her goat (IKK $11 \mathrm{~A}$ cf, Motamedi, A. A. and Lemnart Edelberg 1968). Kabul Museum. Erik Hansen phot. Aug. 1964. 


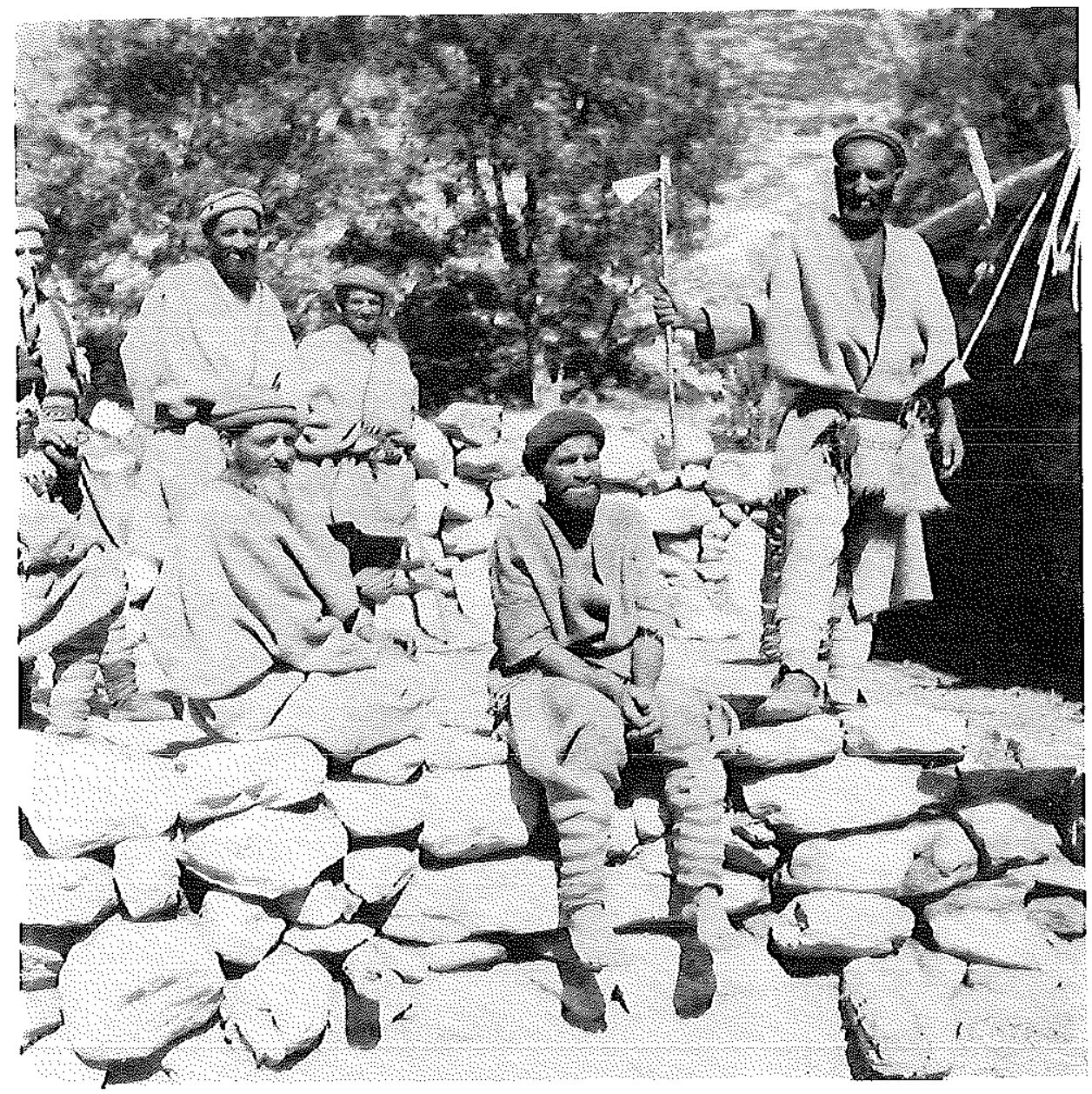

Figure 31. Jan Gul (right) holding his dancing axe. Kushteki. L. E. phot. July 19, 1949. 


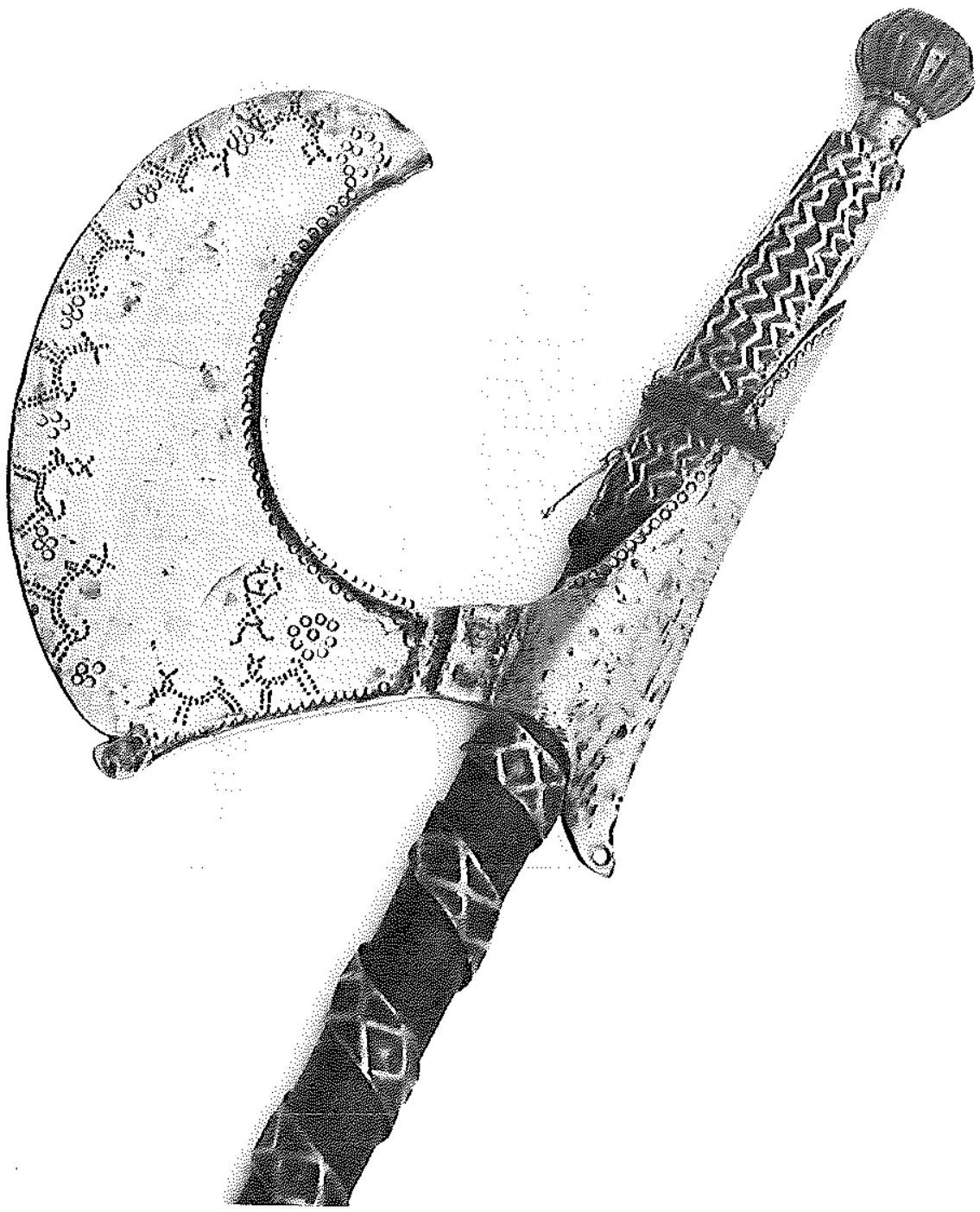

Figure 32. Brass-head of a dancing axe (E. 1709, Moesgard) decorated with gonts having both twisted and curved horns. Behind or over the goats a shepherd (?) presumably initating the twisted horns with his arms, DehHolm phot. 1969. 


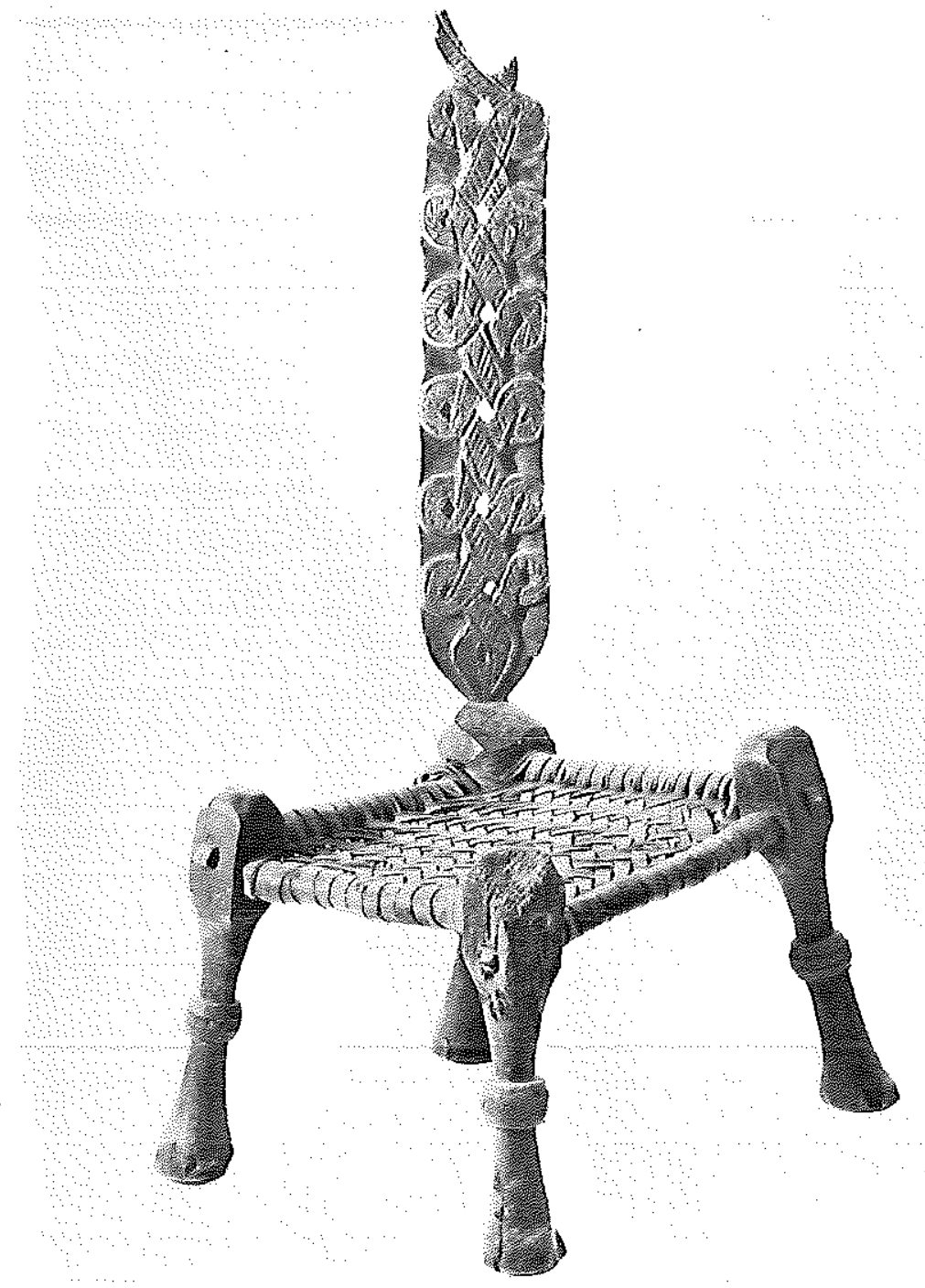

Figure 33. The "horn-chail" (Paruni: sib-kñ) of Kusum (E. A. 99-75, Moesgard). The special backrest of the Parmi horn-chair is formed by one of the legs projecting up above the seat. I have seen three chairs of this kind, one of them without carved designs, and two with a pattern of twisted horns ruming up the backrest (cf. footnotes $92 \& 97$ ). Dehlholm phot. 1969. 


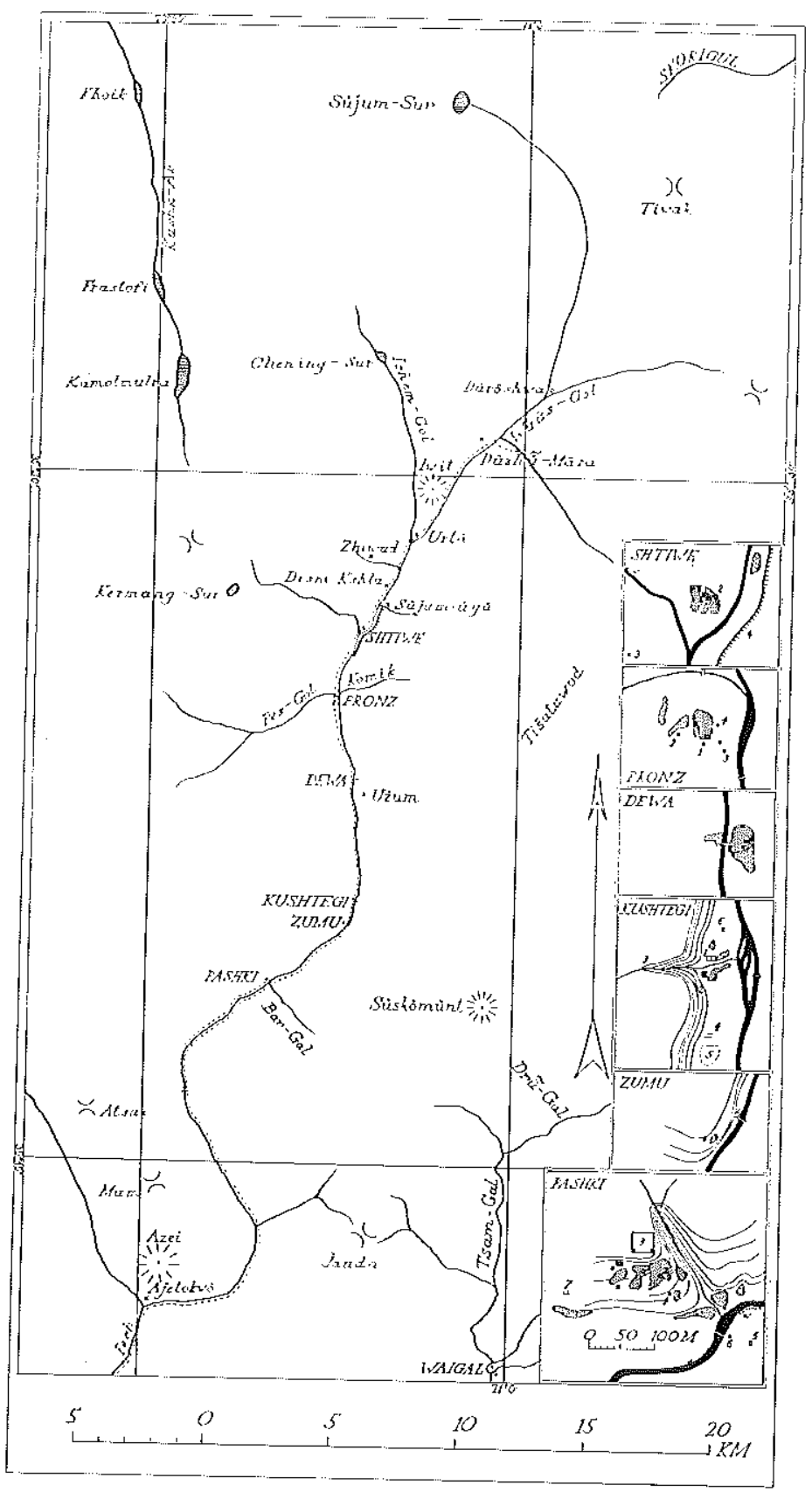

Figure 34. Mythological Map of Partm.

See p. 34-41. The map in Lennart Edelberg and Lis Gramstrup 1971 is more exact from a cartographic point of view. 



\title{
Der zweite Adhyāya des Āśvalāyana-Śrautasūtra, erstmalig vollständig übersetzt und erläutert
}

\author{
VON \\ KLAUS MYLIUS
}

Vorwort.

Der zweite Adhyāya des Āśvalāyana-Śrautasütra ist - unter Berücksichtigung auch des Kommentars - der längste des ganzen Werkes. Dies resultiert aus dem Bemühen des sūtrakāra, nach der Beschreibung des darśapürnamäăa in adhyāya I alle sonstigen havis-Opfer in einem einzigen alhyäya zusammenzufassen. So kommt es, daß die dort herrschende Einheitlichkeit des Sujets im zweiten adhyāya von umso grösserer Mannigfaltigkeit abgelöst wird, wie dies die folgende Übersicht zeigt.

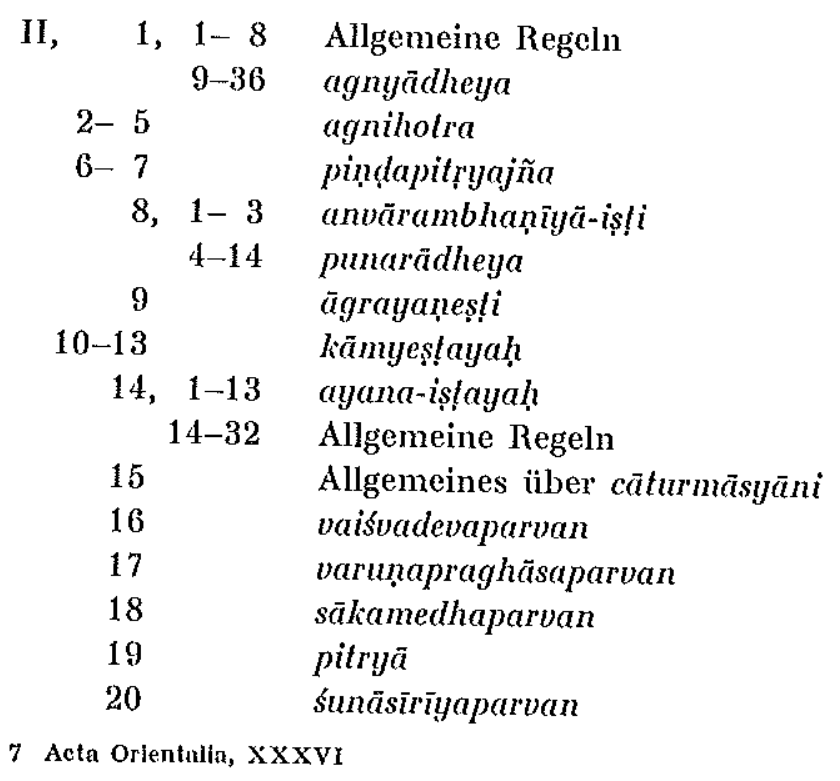


Der erste Adhyãya des AśvŚrS wurde von mir bereits in der Zeitschrift für Missionswissenschaft und Religionswissenschaft (ZMR), Jahrg. 51 (1967), Heft 3-4, übcrsetzt. Auf diese Übersetzung wird, wo crforderlich, kurz mit ZMR Bezug genommen. Auch die dort erläuterten, den Fachkollegen ohmehin vertrauten Abkürzungen wurden beibehalten. Auf Indices wurde jedoch diesmal verzichtet, da diese später als Verzeichnisse zum Gesamtwerk erscheinen sollen.

Quellen und Hilfsmittel sind im großen und ganzen die gleichen geblieben, wie sie im Vorwort zur Übersetzung von adhyāya I genannt wurden. Als wichtige Ergänzung zu Text und Kommentar konnte zusätzlich die Äśvaläyanasütraprayogadipikä benutzt werden.

Auf einige Prinzipien der Übersetzung möge noch kurz eingegangen werden. Bekanntlich bereitet dic formelähnlich gcdrängte Sprache der Sūtras einer sinnvollen Übertragung besonders große Schwierigkeiten. Zur Sicherung der Nachprüfbarkeit wurde daher so wörtlich wie irgend möglich übersetzt. Grammatische und andere simngemäße, von mir herrührende Ergänzungen sind stets eingeklammert. Beabsichtigt war, den wirklichen Inhalt des Sütras zu erfassen, ohne ihn mit den Ansichten der Kommentatoren zu vermischen. Es wurde daher jede aus dem Kommentar bzw. der Prayogadïpika stammende Meinung als solche gekennzeichnet. Vexsuche, den Stoff in eine sprachlich gefällige Form zu kleiden, wurden unterlassen, um die eigentümliche Diktion des Sütra-Stils möglichst getreu viederzugegen. Im übrigen sei auf die im Anmerkungsapparat enthaltenen Erläuterungen verwiesen.

Es ist beabsichtigt, in Abständen die Übersetzung und Erläuterung weiterer Kapitel des Āśvalāyana-Śrautasūtra folgen zu lassen.

II, 1

1. Durch das Vollmondsopfer sind (auch) das iști-, paśu- und soma (-Ritual) erwähnt worden'.

1 Dies ist so zu verstehen, daß das Vollmondopfer als Grundlage u. a. für alle an Agnișoma gerlehteten Rilen gilt. Das triftt vor allem auf das agnişomiga genannte Tieropfer zu, das einen Teil des Somarituals darstellt. Hauptzug sind stets 
2. Mit diesen ${ }^{2}$ opfere man am Neumondstag oder am Vollmondstag ${ }^{3}$.

3. Und ein räjanya möge (dann auch) das agnihotra ${ }^{5}$ darbringen ${ }^{6}$.

4. Einem asketischen Brahmanen ${ }^{7}$ gestatte man, eine andere Zeit zu erwählen ${ }^{8}$.

5. Ein Recht und Wahrheit liebender Somakelterer ${ }^{\theta}$ darf immer opfern ${ }^{10}$.

6. WVenn bei verschiedenen (Riten) Anweisungen über mehrere (Einzelheiten gegeben werden, dann sind diese) in der entsprechenden Reihenfolge (auszuführen).

7. Jedoch bei yajyya $\bar{a}^{11}$ und anwbüky $\bar{a}^{12}$ sind jewcils zwei (Verse an jede Gottheit zu richten ${ }^{13}$ ).

8. Fohlt eine ausdrückliche Anweisung, so sind die beiden üblichen (Verse zu verwenden) ${ }^{14}$.

9. (Nun wird das) agnyädheya ${ }^{15}$ (besprochen).

10. Unter (den Sternbildern ${ }^{10}$ ) kr likikh, rohinï, mrgasiras, den phalgumis, den beiden višákhās, den beiden uttara-proșthapadàs -

11. unter irgendeinem von diesen (ist das agnyädheya $\mathrm{zu}$ vollziehen).

die ajyabhãgau (s. ZMR Anm, 86). Für die işlis (= haviryajna) ist das Vollmondopfer olmehin die Grundform prakrti; vgl. ApSrs XXIV, 3, 32.

${ }^{2}$ Nämlich mit den Grundformen des iști-, pasta* und soma-Rituals.

3 Es sind dafiir also nicht etwa zwel Tage in Anspruch zu nehmen (Pray.).

- Her - kşatriya (Angehöriger des zweiten varna); vgl. KS XXVII, 1; AB VIII, 2, 1; SB V, 1, 1, 11. 5, 3.5.9.12.28; XIII, 1, 5, 3. 6, 2, 10 ,

5 Vgl, unten II, 2-5.

- Das gilt nach dem Komm. auch für einen vaisya.

? Um die Exklusivität des Brahmanenstandes zu unterstreichen.

8 Nämlich für das agnihotra.

$\theta=$ Somapferer.

10 Nämllch das agnihotra.

11 S. ZMR Amm. 162.

12 S. ZMR Anm. 195.

13 IKonm,

14 Dieses sūtra blebt unklar; auch der Komm. weiß sich nicht zu entscheiden.

15 Die als einer der haviryajñas kłassiffzierte Anlegung der heiligen Feuer.

${ }^{16} \mathrm{~S}$. MacDonell-Keith: Vedic Index of Names and Subjects (1912), sub nakşatra. 
12. Ein Brahmane möge (die heiligen Feuer) im Frühling zu (einem der) Mondwechsel errichten, -

13. cin lssatriya im Sommer, ein vaiśya in der Regenzeit, ein Zimmermann $^{17}$ im Herbst.

14. Man kann (dic Feuer) auch in jeder beliebigen Jahreszeit anlegen $^{18}$.

15. Wer (nach dem agnyădheya) ein Somaopfer darzubringen wünscht, braucht sich weder um die Jahreszeit noch $\mathrm{um}$ die Sternkonstellation zu kümmern.

16. $\mathrm{Er}^{19}$ bringe die aus aśvallha $a^{20}$ und einem śami ${ }^{21}-\mathrm{Sproß}$ (bestehenden) beiden Reibhölzer ${ }^{22}$ heran, ohne sich umzusehen (und spreche:)

17. „Der aśvallha, der śamï-Sproß ${ }^{23}$, der mit dir zusammen emporgewachsen ist ${ }^{24}$, den bringe ich dir mit einem Segensspruche her ${ }^{25}$, zusammen mit den opferwïrdigen Kennzeichen $^{26}$.

Die Opferung eines vollen Löfels Schmelzbutter steht am Ende des agnyäalheya ${ }^{27}$.

18. Wemn sich (aber) noch iști (-Opfer) daran schließen sollen, -

19. dann (sind) bei der ersten (iș/i) Agni und Agni Pavamăna (die angerufenen Gotheiten).

20. (Für Agni Pavamāna ${ }^{28}$ rezitiert der hotar:) "Agni, clu läuterst die Leben ${ }^{29}$; $"$ Agni, gieße aus, Geschickter $\ll^{30}$.

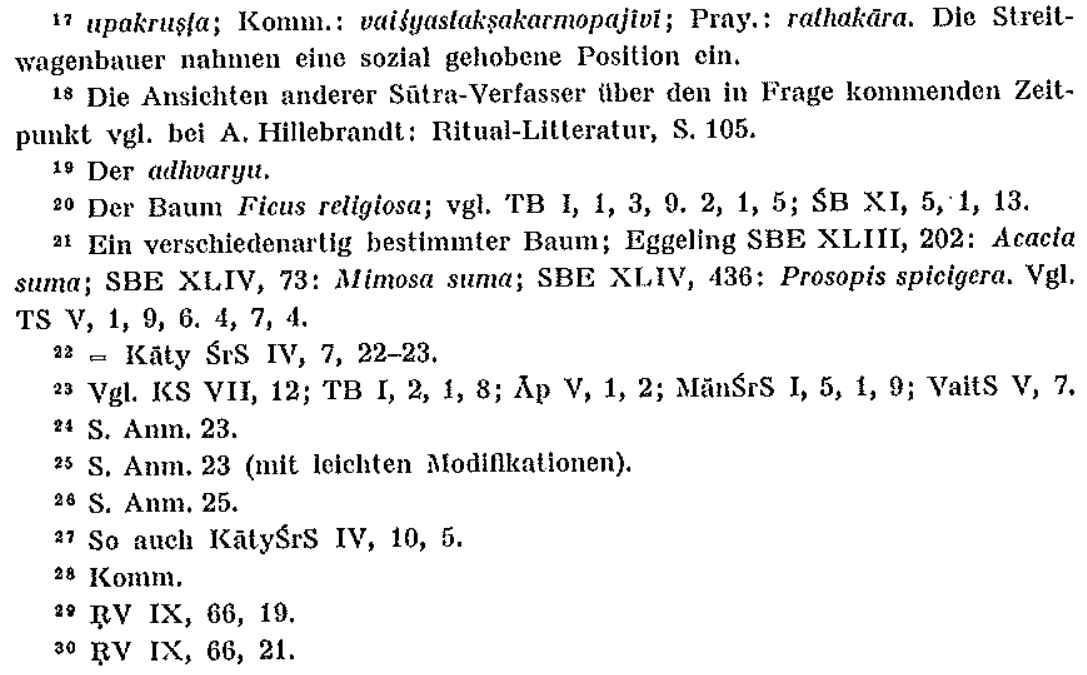

